\title{
The Wolf-Rayet stars in the Large Magellanic Cloud
}

\section{A comprehensive analysis of the WN class $^{\star}, \star \star$}

\author{
R. Hainich ${ }^{1}$, U. Rühling ${ }^{1}$, H. Todt ${ }^{1}$, L. M. Oskinova ${ }^{1}$, A. Liermann ${ }^{2}$, G. Gräfener ${ }^{3}$, C. Foellmi ${ }^{4}$, \\ O. Schnurr ${ }^{2}$, and W.-R. Hamann ${ }^{1}$
}

\author{
${ }^{1}$ Institut für Physik und Astronomie, Universität Potsdam, Karl-Liebknecht-Str. 24/25, 14476 Potsdam, Germany \\ e-mail: rhainich@astro.physik.uni-potsdam.de \\ ${ }^{2}$ Leibniz-Institut für Astrophysik Potsdam, An der Sternwarte 16, 14482 Potsdam, Germany \\ 3 Armagh Observatory, College Hill, Armagh BT6 9D, UK \\ 412 rue Servan, 38000 Grenoble, France
}

Received 17 September 2013 / Accepted 16 January 2014

\begin{abstract}
Context. Massive stars, although being important building blocks of galaxies, are still not fully understood. This especially holds true for Wolf-Rayet (WR) stars with their strong mass loss, whose spectral analysis requires adequate model atmospheres.

Aims. Following our comprehensive studies of the WR stars in the Milky Way, we now present spectroscopic analyses of almost all known WN stars in the LMC.

Methods. For the quantitative analysis of the wind-dominated emission-line spectra, we employ the Potsdam Wolf-Rayet (PoWR) model atmosphere code. By fitting synthetic spectra to the observed spectral energy distribution and the available spectra (ultraviolet and optical), we obtain the physical properties of 107 stars.

Results. We present the fundamental stellar and wind parameters for an almost complete sample of WN stars in the LMC. Among those stars that are putatively single, two different groups can be clearly distinguished. While $12 \%$ of our sample are more luminous than $10^{6} L_{\odot}$ and contain a significant amount of hydrogen, $88 \%$ of the WN stars, with little or no hydrogen, populate the luminosity range between $\log \left(L / L_{\odot}\right)=5.3 \ldots 5.8$.

Conclusions. While the few extremely luminous stars $\left(\log \left(L / L_{\odot}\right)>6\right)$, if indeed single stars, descended directly from the main sequence at very high initial masses, the bulk of WN stars have gone through the red-supergiant phase. According to their luminosities in the range of $\log \left(L / L_{\odot}\right)=5.3 \ldots 5.8$, these stars originate from initial masses between 20 and $40 M_{\odot}$. This mass range is similar to the one found in the Galaxy, i.e. the expected metallicity dependence of the evolution is not seen. Current stellar evolution tracks, even when accounting for rotationally induced mixing, still partly fail to reproduce the observed ranges of luminosities and initial masses. Moreover, stellar radii are generally larger and effective temperatures correspondingly lower than predicted from stellar evolution models, probably due to subphotospheric inflation.
\end{abstract}

Key words. stars: Wolf-Rayet - Magellanic Clouds - stars: early-type - stars: atmospheres - stars: winds, outflows - stars: mass-loss

\section{Introduction}

The Large Magellanic Cloud (LMC) is one of the closest galaxies to the Milky Way (MW), allowing detailed spectroscopy of its brighter stars. Its distance modulus of only $D M=18.5 \mathrm{mag}$ is well constrained (Madore \& Freedman 1998; Pietrzyński et al. 2013). Another advantage in analyzing stars of the LMC is the marginal reddening along the line of sight (Subramaniam 2005; Haschke et al. 2011), which is in general below $E_{b-v}=0.25 \mathrm{mag}$ (Larsen et al. 2000).

Compared to our Galaxy, the LMC is much smaller and has a deviating structure that is intermediate between a dwarf spiral and an irregular type. The LMC exhibits a very different

\footnotetext{
* Partly based on observations made with the NASA/ESA Hubble Space Telescope, and obtained from the Hubble Legacy Archive, which is a collaboration between the Space Telescope Science Institute (STScI/NASA), the Space Telescope European Coordinating Facility (ST-ECF/ESA), and the Canadian Astronomy Data Centre (CADC/NRC/CSA).

$\star \star$ Appendices $\mathrm{A}-\mathrm{C}$ are available in electronic form at http://www . aanda.org
}

history of star formation than the MW. The metallicity observed in LMC stars is, in general, subsolar $\left(Z / Z_{\odot} \sim 0.4\right.$, Dufour et al. 1982), but with a strong age-dependence (e.g., Piatti \& Geisler 2013). For young massive stars, it may reach nearly solar values. In the stellar evolution calculations that we will discuss below, Meynet \& Maeder (2005) adopted $Z=0.008$, which is about $60 \%$ of the solar value (Asplund et al. 2009).

The metallicity is expected to have significant influence on the evolution of massive stars as it has impact on the mass loss due to stellar winds. As far as these winds are driven by radiation pressure on spectral lines of metals like iron, the mass-loss rate is expected to scale with $Z^{m}$ with $m \approx 0.5$ (e.g., Kudritzki et al. 1989). Based on their theoretical models, Vink \& de Koter (2005) derived an exponent of $m=0.86$ for late-type WolfRayet (WR) stars. The fact that the distribution of the WR stars on the subclasses (i.e., the nitrogen sequence: $\mathrm{WN}$ and the carbon sequence: WC) strongly differs between the LMC and the MW is generally attributed to this metallicity effect. Eldridge \& Vink (2006) found that the mass-loss rates from Vink \& de Koter (2005) can account for the observed WC/WN ratio as a function of the metallicity. The metallicity dependence of the 
WN mass-loss was affirmed by the hydrodynamic stellar wind models presented by Gräfener \& Hamann (2008).

In previous papers, we have concentrated on analyzing the WR population of the MW. In Hamann et al. (2006, hereafter HGL06), we presented a comprehensive analysis of the Galactic WN stars, while the WC subtypes were studied by Sander et al. (2012). For both classes we found discrepancies between the parameters of the observed WR population and the predictions of the available stellar evolution calculations.

The current paper focuses on the WR stars in the LMC. With more than 100 objects, our sample comprise nearly all WN-type stars known in the LMC. In contrast, earlier analyses of WN stars in the LMC were limited to a sample size below 20 objects (Crowther \& Smith 1997; Crowther \& Dessart 1998; Hamann \& Koesterke 2000) and were often confined to specific subclasses (Crowther et al. 1995a; Pasquali et al. 1997).

At the time of these studies, stellar atmosphere models commonly did not yet account for iron-line blanketing (Hillier \& Miller 1999; Gräfener et al. 2002) and wind inhomogeneities. The inclusion of these two effects, the latter by means of the microclumping approach (cf. Hamann \& Koesterke 1998), significantly improved stellar atmosphere models and entailed a pervasive revision of the derived stellar parameters (e.g., Hamann \& Koesterke 2000; Crowther et al. 2002, 2010; Sander et al. 2012). Similar profound improvements were achieved in the field of stellar evolution by the inclusion of physical processes such as stellar rotation (Meynet \& Maeder 2003, 2005) and, more recently, magnetic fields (Maeder \& Meynet 2005; Yoon et al. 2012).

In the last decade, high signal to noise spectra in the optical spectral range of almost all WN stars in the LMC were obtained in extensive spectroscopic studies realized by Foellmi et al. (2003b) and Schnurr et al. (2008). For the first time, the spectra obtained by these two studies make it possible to analyze a comprehensive sample of LMC WN stars. By this means, we obtain a general overview of a nearly complete WN-star population, which we employ to test state-of-the-art evolution models.

This paper is organized as follows: in the next section, we introduce our sample of stars and the observational data employed. In Sect.3, we briefly characterize the Potsdam WolfRayet (PoWR) model atmospheres. The method of our analyses is described in Sect. 4. The results are compiled in Sect. 5. In Sect. 6, we discuss our results with respect to the stellar evolution theory. A summary and conclusions are presented in Sect. 7.

The appendices give details about the observational data (Appendix A) and comments on the individual stars (Appendix B). Finally, we provide spectral fits for all sample stars (Appendix C).

\section{The sample}

\subsection{Sample selection}

Our sample is based on the fourth catalog of WR stars in the LMC (Breysacher et al. 1999, hereafter BAT99). Throughout this paper, we identify the stars by their running number in that list. In this catalog, a spectral type of the WN sequence was assigned to each of the 109 objects.

In a few cases, the spectral classification had to be revised. The stars BAT99 45 and BAT99 83 are actually luminous blue variables (LBVs; see Humphreys \& Davidson 1994; Schnurr et al. 2008) and are, therefore, excluded from our sample.

Five of the stars listed with a WN classification in the BAT99 catalog have been reclassified as Of-types: BAT99 107 has been identified as a massive spectroscopic binary system comprising two Of-type stars (Taylor et al. 2011). Niemela et al. (2001) found BAT99 6 to be an O-type binary system as well. Crowther \& Walborn (2011) have reclassified BAT99 105 and BAT99 110 as O2If* stars. The spectral type O3If* has been assigned to BAT99 93 by Evans et al. (2011). Despite their reclassifications, we keep these O-type objects in our sample. Thus, the number of proper WN stars from the original BAT99 catalog is reduced to 102 .

Since the publication of the BAT99 catalog, only a few additional WN stars have been identified in the LMC. A list of the seven newly discovered WR stars, six of them WN-type stars, can be found in Table 3 of Neugent et al. (2012). Thus, the number of known WN stars in the LMC amounts to 108, although two of these new detections are precarious. Massey et al. (2000) identified Sk-69 194 as B0 Ia + WN. However, Foellmi et al. (2003b) could not confirm this detection. Neugent et al. (2012) also list LH 90 $\beta$-6 as a new WN star. However, according to Massey et al. (2000), this is an alias of TSWR 1, which was resolved into multiple components by Walborn et al. (1999) and incorporated in the BAT99 catalog with the number BAT9978. Therefore, the basis of this new detection is not clear. Another six new WR stars in the LMC are reported by Reid \& Parker (2012), but without giving coordinates or closer classifications. We do not include any of these newly discovered WN stars in our analyses.

With 102 out of 108 known WN stars in the LMC, our sample covers this class nearly completely with all subtypes present. The spatial distribution of our program stars is illustrated in Fig. 1, and the complete list of analyzed objects (including the five Of stars) is compiled in Table 2. For the majority of our sample, the spectral types have been determined by Foellmi et al. (2003b) and Schnurr et al. (2008), respectively, based on the classification scheme elaborated by Smith et al. (1996). For the handful of stars missing in their samples, we adopt the spectral type from the BAT99 catalog. A couple of our stars have been reclassified by various authors since the publication of these catalogs. The present classification of each star is quoted in Table 2. The subtypes WN2 to WN5 are sometimes referred to as WNE ("early"), while WN6 to WN11 are referred to as WNL ("late").

The total census of WR stars in the LMC, as far as they are assigned to their subclass, amounts to 134. In addition to the $108 \mathrm{WN}$ stars, only $24 \mathrm{WC}$ stars plus two WR stars with prominent oxygen lines (WO stars) were discovered (Barlow \& Hummer 1982; Neugent et al. 2012). The composition of the WR population is thus very different from our Galaxy, where the ratio of $\mathrm{WN}$ to $\mathrm{WC}$ stars is close to unity.

\subsection{Binaries}

Among the objects in our sample some may be binary (or multiple) systems. We, therefore, carefully consider the binary status of each object. All stars for which Foellmi et al. (2003b), Schnurr et al. (2008), or BAT99 list periodic radial-velocity variations are considered as confirmed binaries (cf. Table 2).

For some of our targets there are less conclusive radialvelocity measurements or binary classifications based on spectral peculiarities. Such cases are considered binary suspects, as indicated by a question mark in Table 2 with the corresponding references.

Another method to identify WR stars as binaries is to evaluate their X-ray luminosity. According to studies of Galactic WR-stars, single WC-type stars are not X-ray sources at all (Oskinova et al. 2003), while single WN-type stars in general 


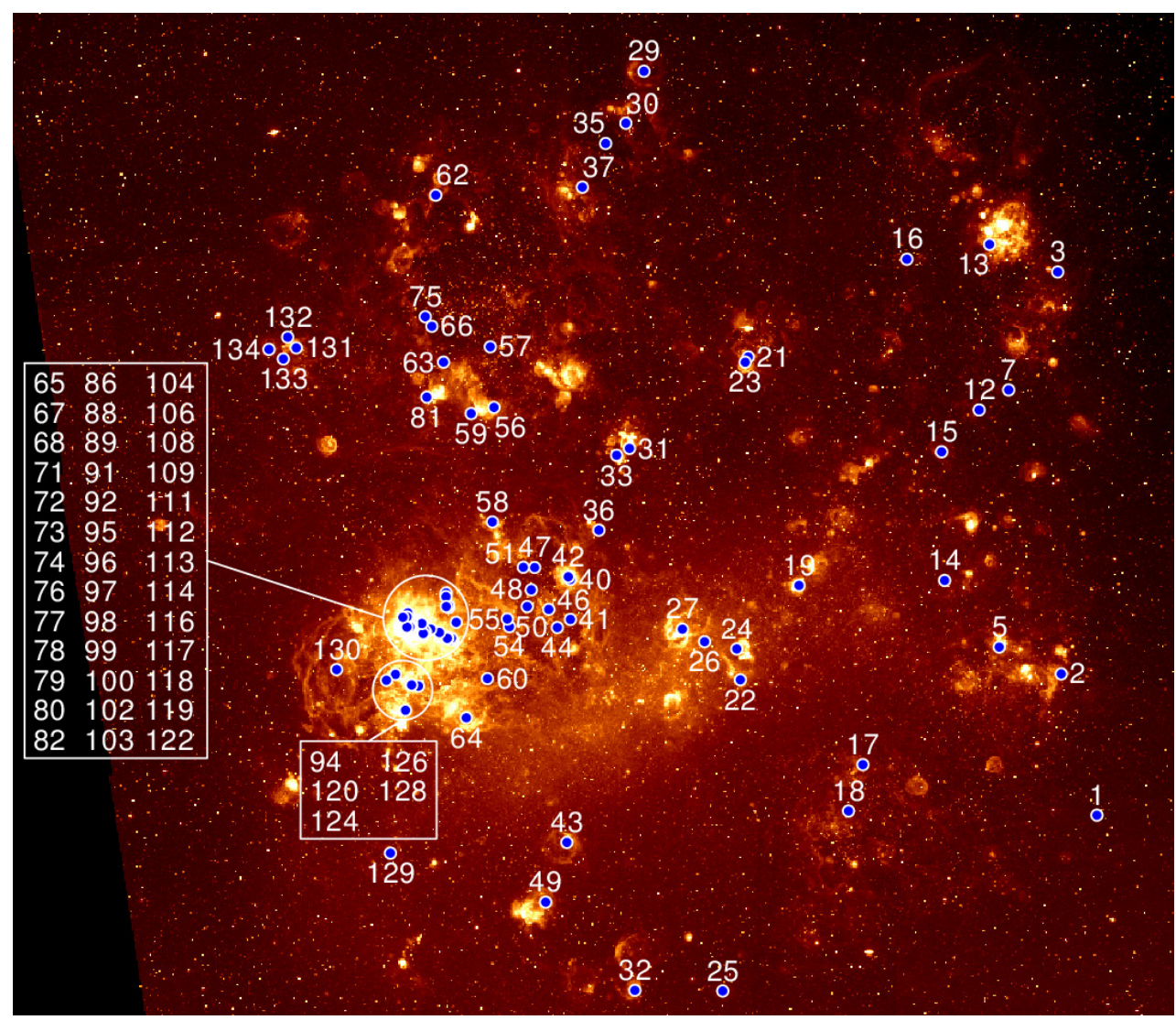

Fig. 1. WN stars of our sample, identified by their number in the BAT99 catalog. The two boxes refer to the very crowded region of 30 Doradus. The $\mathrm{H} \alpha$ image in the background is from the Magellanic Cloud Emission-Line Survey (MCELS, Smith et al. 2005).

are relatively X-ray faint. Some of them remain undetected in $\mathrm{X}$-rays despite quite sensitive observations, setting strict upper limits on the X-ray luminosity. For example, Gosset et al. (2005) obtained $L_{\mathrm{X}}<2 \times 10^{30} \mathrm{erg} \mathrm{s}^{-1}$ for WR 40 (WN8). The X-ray luminosities of those WN stars that were detected are relatively small, not exceeding a few times $10^{32} \mathrm{erg} \mathrm{s}^{-1}$ (Ignace et al. 2000, 2003; Skinner et al. 2012). The mechanism of X-ray production in single WN stars is not fully understood, but is thought to be related to the presence of clumps and large scale structures in their winds (Chené et al. 2011; Oskinova et al. 2012).

In contrast, binary WR stars with colliding winds are significantly more X-ray luminous than single stars. In such systems, the production of X-rays is explained by the heating of gas in a strong shock that results when two stellar winds collide (Stevens et al. 1992). Therefore, a higher than usual X-ray luminosity can serve as a good indicator for a colliding wind binary. As an example, the high X-ray luminosity of the Galactic star WR $25\left(L_{\mathrm{X}}=1.3 \times 10^{34} \mathrm{erg} \mathrm{s}^{-1}\right)$ provided strong indications that this star is a colliding-wind binary (Raassen et al. 2003), as confirmed later from radial velocity measurements (Gamen et al. 2006).

Binary WR-stars with a compact companion, i.e., with a neutron star or black hole, are expected to have even higher X-ray luminosities exceeding $10^{35} \mathrm{erg} \mathrm{s}^{-1}$. The $\mathrm{X}$-ray luminosities in these systems are powered by the wind accretion onto the companion. An intriguing example is Cyg X-3, a Galactic high-mass $\mathrm{X}$-ray binary with a WN-type primary, which has an X-ray luminosity of $10^{38} \mathrm{erg} \mathrm{s}^{-1}$ (e.g., Lommen et al. 2005).

The X-ray properties of WR stars in the Magellanic Clouds were studied systematically by Guerrero \& Chu (2008a,b), using observations with the X-ray observatories ROSAT and Chandra. The sensitivity of these surveys was limited to X-ray luminosities of a few times $10^{32} \mathrm{erg} \mathrm{s}^{-1}$. They detected X-rays from 27 of the WR stars in the LMC, with X-ray luminosities being similar to those of Galactic colliding-wind binaries. Since there is no reason to assume that single WR-stars in the LMC are intrinsically more bright in X-rays than in the Galaxy, we suspect all WR stars detected by Guerrero \& Chu to be colliding wind binaries and mark them accordingly in Table 2.

Altogether, our sample (without the Of stars) includes 17 confirmed binaries plus 22 binary suspects. From 108 known WN stars in the LMC, this corresponds to a binary frequency of only $16-36 \%$. Although this binary fraction seems to be a bit low, it is in line with expectations from binary population studies (e.g., Foellmi et al. 2003a; Chini et al. 2012; Sana et al. 2012, 2013a). Moreover, there are most likely more binaries in our sample that are not yet recognized.

\subsection{Observational data}

This study was facilitated by optical spectra obtained by Foellmi et al. (2003b) who observed 61 WNE stars with various instruments between 1998 and 2002. These data are publicly available $^{1}$. The completeness of this study was only possible due to spectroscopic observations of 42 late-type WN stars carried out by Schnurr et al. (2008). For details on the instrumentation and data reduction, we refer to Foellmi et al. (2003b) and Schnurr et al. (2008). These two sets of data were primarily designed to

\footnotetext{
http://wikimbad.obs.ujf-grenoble.fr/Category_ Wolf-Rayet_Star.html
} 
search for radial velocity variations, the results being published in Foellmi et al. (2003b) and Schnurr et al. (2008). These spectra were not flux-calibrated and have been normalized by the respective authors.

From the VizieR archive we retrieved flux-calibrated, lowresolution optical spectra for most of our targets, recorded by Torres-Dodgen \& Massey (1988) on a SIT-vidicon detector at the Cassegrain spectrograph of the $1.5 \mathrm{~m}$ telescope of the Cerro Tololo Inter-American Observatory (CTIO). Furthermore, we reused 19 observations dating back to 1989 (cf. Koesterke et al. 1991), obtained with the the ESO Faint Object Spectrograph and Camera (EFOSC) at the $3.6 \mathrm{~m}$ telescope. Unreduced spectra of WN and Of stars observed with the Anglo-Australian Telescope (AAT) were obtained from the study by Crowther \& Smith (1997). We performed the wavelength calibration with given arc lamp data and normalized them "by eye" if no other optical spectra were at hand.

Ultraviolet spectra secured with the International Ultraviolet Explorer (IUE) are available from the archives for almost all of the stars of our sample, except for those located in the very crowded 30 Dor region. Especially for some of the latter, UV and optical spectra were recorded with spectrographs aboard the Hubble Space Telescope (HST). A subset of 19 stars have been observed with the Far Ultraviolet Spectroscopic Explorer (FUSE), but these data were not used in the current study. The FUSE spectra will be the subject of a detailed abundance analysis of LMC WN stars in a subsequent paper.

Before fitting the observed spectra, we corrected the wavelengths for the radial velocities of the individual stars, mostly taken from Foellmi et al. (2003b) and Schnurr et al. (2008). The details about the origin of all spectra employed in this paper are compiled in Table A.1.

We used narrowband optical photometry $(u, b, v)$ obtained by Crowther \& Hadfield (2006) whenever available. Otherwise, we used the older measurements from Torres-Dodgen \& Massey (1988), and finally complemented the data with values from BAT99. Near-infrared magnitudes $\left(J, H, K_{\mathrm{S}}\right)$ were retrieved from the 2MASS catalog (Skrutskie et al. 2006), except for those stars located in the crowded field of 30 Dor. Photometry from the InfraRed Array Camera (IRAC, 3.6, 4.5, 5.8, and $8.0 \mu \mathrm{m}$ ) of the Spitzer Space Telescope is available for most stars from the catalog by Bonanos et al. (2009).

\section{The models}

Our spectral analyses are based on non-local thermodynamic equilibrium (non-LTE) model atmospheres calculated with the PoWR code. Its basic assumptions are spherical symmetry and stationarity of the flow. The radiative transfer equation is solved in the comoving frame, iteratively with the equations of statistical equilibrium and radiative equilibrium. For more details of the PoWR code, see Hamann \& Gräfener (2004).

The main parameters of a model atmosphere are the luminosity $L$ and the "stellar temperature" $T_{*}$. The latter is the effective temperature related to the stellar radius $R_{*}$ via the StefanBoltzmann law

$L=4 \pi \sigma R_{*}^{2} T_{*}^{4}$.

The stellar radius $R_{*}$ is per definition located at a radial Rosseland optical depth of 20 , which represents the lower boundary of the model atmosphere.

Additional parameters, which describe the stellar wind, can be combined in the so-called transformed radius $R_{\mathrm{t}}$. This quantity was introduced by Schmutz et al. (1989); we define it as

$R_{\mathrm{t}}=R_{*}\left(\frac{v_{\infty}}{2500 \mathrm{~km} \mathrm{~s}^{-1}} / \frac{\dot{M} \sqrt{D}}{10^{-4} M_{\odot} \mathrm{yr}^{-1}}\right)^{2 / 3}$

with $v_{\infty}$ denoting the terminal wind velocity, $\dot{M}$ the mass-loss rate, and $D$ the clumping contrast (see below). Schmutz et al. (1989) noticed that model spectra with equal $R_{\mathrm{t}}$ exhibit approximately the same emission line strengths, independent of the specific combination of the particular wind parameters as long as $T_{*}$ and the chemical composition are the same. Even the line profile is conserved under the additional condition that $v_{\infty}$ is also kept constant. One can understand this invariance when realizing that $R_{\mathrm{t}}$ is related to the ratio between the volume emission measure and the stellar surface area.

According to this scaling invariance, a model can be scaled to a different luminosity as long as $R_{\mathrm{t}}$ and $T_{*}$ are unchanged. Equation (2) implies that the mass-loss rate then must be scaled proportional to $L^{3 / 4}$ in order to preserve the normalized line spectrum.

Allowing for wind inhomogeneities, the "density contrast" $D$ is the factor by which the density in the clumps is enhanced compared to a homogeneous wind of the same $\dot{M}$. We account for wind clumping in the approximation of optically thin structures (Hillier 1991; Hamann \& Koesterke 1998). From the analysis of the electron-scattering line wings in Galactic WN stars, Hamann \& Koesterke (1998) found that a density contrast of $D=4$ is adequate. To the contrary, Crowther et al. (2010) and Doran et al. (2013) inferred $D=10$ in their analyses of WN stars in the 30 Doradus region. For the current study, we uniformly adopt a density contrast of $D=10$, because we noticed in a detailed investigation of a subsample that with $D=4$, the line-scattering wings in the models are stronger than observed. Note that the empirical mass-loss rates derived in this work scale with $D^{-1 / 2}$ (cf. Eq. (2)).

For the Doppler velocity $v_{\mathrm{D}}$, describing the line broadening due to microturbulence and thermal motion, we adopt a value of $100 \mathrm{~km} \mathrm{~s}^{-1}$, which provides a good fit to the data and is approved in previous studies (e.g., Hamann \& Koesterke 2000, hereafter HK2000; HGL06).

For the velocity law $v(r)$ in the supersonic part of the wind, we adopt the so-called $\beta$-law. For the exponent $\beta$, the radiationdriven wind theory predicts about 0.8 in agreement with observations (e.g., Pauldrach et al. 1986). In WN stars, the law is more shallow because of multiple-scattering effects. We adopt $\beta=1$, which better resembles the hydrodynamic prediction (Gräfener \& Hamann 2007), and yields consistent spectral fits. In the subsonic part, the velocity field is implied by the hydrostatic density stratification according to the continuity equation.

The models are calculated using complex atomic data of $\mathrm{H}$, $\mathrm{He}, \mathrm{C}$, and N. Iron group elements are considered in the "superlevel approach" that encompasses $\sim 10^{7}$ line transitions between $\sim 10^{5}$ levels within 72 superlevels (Gräfener et al. 2002).

\section{Method}

To facilitate the analysis of a large number of WN stars, we first establish grids of models. As explained above, the main parameters are the stellar temperature $T_{*}$ and the transformed radius $R_{\mathrm{t}}$.

\subsection{Abundances}

We calculated three grids of models for different hydrogen abundances: one hydrogen-free "WNE" grid and two "WNL" 
Table 1. Chemical composition (mass fractions in percent).

\begin{tabular}{lcccccc}
\hline \hline & Sun $^{a}$ & Gal. & \multicolumn{4}{c}{ LMC } \\
\cline { 4 - 7 } & & WN $^{b}$ & B stars $^{c}$ & B stars $^{d}$ & H II $^{e}$ & WN $^{f}$ \\
\hline $\mathrm{C}$ & 0.237 & 0.01 & 0.054 & 0.086 & 0.058 & 0.0067 \\
$\mathrm{~N}$ & 0.069 & 1.5 & 0.0083 & 0.011 & 0.0087 & 0.40 \\
$\mathrm{O}$ & 0.573 & - & 0.27 & 0.30 & 0.281 & - \\
$\mathrm{LCNO}$ & 0.88 & 1.5 & 0.33 & 0.40 & 0.35 & 0.41 \\
$\mathrm{Fe}$ & 0.129 & $0.14^{g}$ & $0.07^{h}$ & - & - & $.07^{g}$ \\
\hline
\end{tabular}

Notes. ${ }^{(a)}$ Asplund et al. (2009); ${ }^{(b)}$ as used in HGL06; ${ }^{(c)}$ Hunter et al. (2007); (d) Korn et al. (2005); ${ }^{(e)}$ H II regions (Kurt \& Dufour 1998); $(f)$ as adopted in this work; ${ }^{(g)}$ including the whole iron group; ${ }^{(h)}$ mean value from Trundle et al. (2007).

grids with hydrogen mass fractions of 0.2 and 0.4 , respectively. From the trace elements, we account for carbon, nitrogen, and a generic model atom representing the iron-group elements in relative solar mixture (Gräfener et al. 2002).

The material in the $\mathrm{WN}$ atmosphere has undergone at least partial CNO burning due to mixing processes in the stellar interior, such as rotational induced mixing (e.g., Heger \& Langer 2000). Accordingly, most of the oxygen and carbon was transformed into nitrogen. Assuming equilibrium, the remaining mass fractions of oxygen and carbon relative to that of nitrogen should be only $1 / 60$ (Schaerer et al. 1993). Hence, the nitrogen abundance should roughly equal the sum of the $\mathrm{C}, \mathrm{N}$, and $\mathrm{O}$ abundances of the initial material from which the star was formed. Note that HK2000 inferred a nitrogen abundance for the Galactic WN stars nearly twice the sum of solar CNO (cf. Table 1).

As a reference for LMC abundances, we use spectral analyses of B-type stars. The results from two such studies (Hunter et al. 2007; Korn et al. 2005) are listed in Table 1. Moreover, the table gives abundances found in H II regions of the LMC (Kurt \& Dufour 1998). Compared to solar abundances, the sum of C, N, and $\mathrm{O}$ in these LMC objects is roughly half the solar value. We, therefore, adopt a nitrogen mass fraction of 0.004 for the models throughout this paper. The carbon abundance is set to $1 / 60$ of this value. We neglect oxygen in our WN star models, since no prominent $\mathrm{O}$ lines are present in the optical wavelength range, nor do we expect it to influence the atmospheric stratification.

The iron abundance in B-type stars from several clusters in the LMC has been studied by Trundle et al. (2007) (see Table 1). On average, this value is 0.0007 (mass fraction), i.e., again roughly half the solar iron abundance. We adopted this value as the iron-group abundance for our LMC models.

A detailed abundance analysis is beyond the scope of the present paper. However, the spectral fits presented below reveal that the models cannot reproduce the observed nitrogen lines for a subset of our sample. Thus, it seems that the $\mathrm{N}$ abundance in these LMC WN stars is slightly higher than our adopted value.

\subsection{The model grids}

Three large grids of WN models were computed. The parameter domain of each grid is spanned by $T_{*}$ and $R_{\mathrm{t}}$. The grid spacing is $0.05 \mathrm{dex}$ in $\log \left(T_{*} / \mathrm{kK}\right)$ and $0.1 \mathrm{dex}$ in $\log \left(R_{\mathrm{t}} / R_{\odot}\right)$ (see Hamann \& Gräfener 2004, for details). The luminosity is fixed at $\log \left(L / L_{\odot}\right)=5.3$. Thanks to the scaling invariance described in Sect. 3, the normalized line spectra apply in good approximation to different luminosities, while the absolute fluxes scale with $L$.

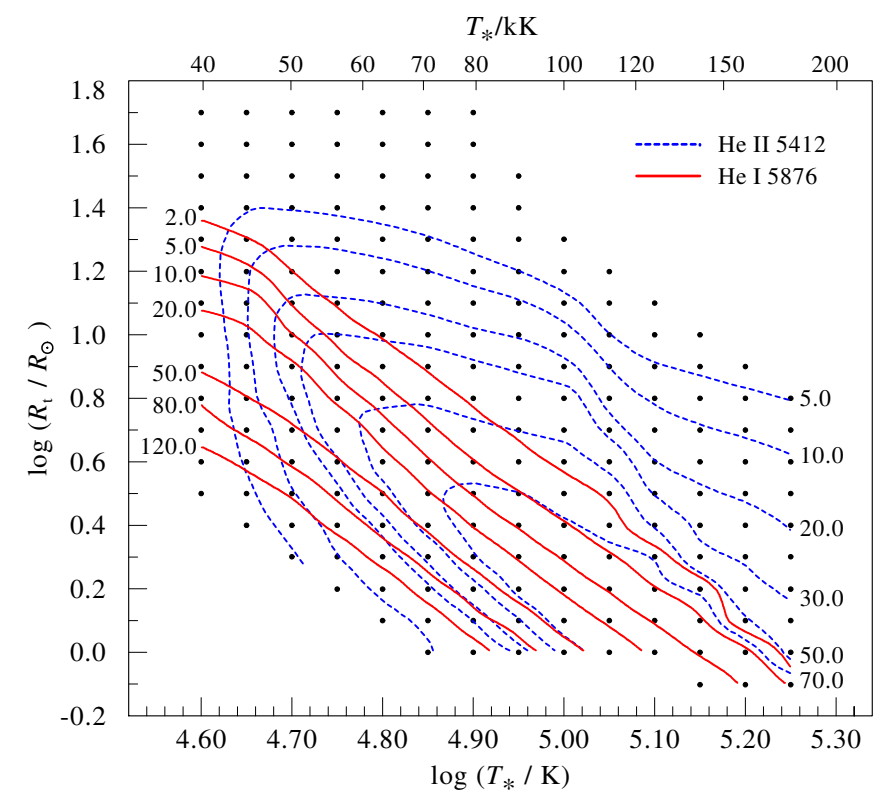

Fig. 2. Grid of models for hydrogen-free WN stars in the LMC: contours of constant line emission, labeled with the equivalent width in $\AA$; thick (red) contours: He I line at $5876 \AA$, thin-dashed (blue) contours: He II at $5412 \AA$. Tiny dots indicate calculated grid models.

The three grids differ not only in the hydrogen mass fraction $(0,0.2$, and 0.4 , respectively), but also in the terminal wind velocity. One grid has been computed for WNE stars with $X_{\mathrm{H}}=0$ and $v_{\infty}=1600 \mathrm{~km} \mathrm{~s}^{-1}$. The other two grids were established for WNL stars $\left(X_{\mathrm{H}}=0.2\right.$ and $\left.X_{\mathrm{H}}=0.4\right)$, respectively, with $v_{\infty}=1000 \mathrm{~km} \mathrm{~s}^{-1}$ in both cases. The trace element abundances are set to the values described in Sect. 4.1. These model grids are publicly available on our website ${ }^{2}$.

Analyzing a star thus means to identify the specific model which gives the best fit to the observations. A first orientation can be obtained from contour plots like the one shown in Fig. 2. If, for example, the He II emission line at $5412 \AA$ is observed with an equivalent width of $30 \AA$, the temperature is restricted to values above $50 \mathrm{kK}$. Combining this with the measured equivalent width of the He I line at $5876 \AA$, preliminary model parameters can already be estimated from the intersection point of the corresponding contours.

This method works, of course, only if the He I and the He II lines are both present in the spectrum of the considered star. For those stars where this is not the case, such as very hot stars, other ions or elements must be employed. These contour plots are provided on the PoWR homepage for several transitions of the ions He I, He II, N III, N IV, and N V. As is evident from Fig. 2, the method may also fail in the lowest part of the diagram, i.e., for the densest winds, because the contours do not intersect in this parameter regime. At these parameters, the winds are so thick that the whole spectrum, including the continuum, is formed in the rapidly moving part of the wind. For a fixed luminosity, such models have only the mass-loss rate as significant parameter, while the stellar radius and the related effective temperature become meaningless. Due to the spacing chosen for our grid, models of the same mass-loss rate lie on a diagonal of the grid cells, like the parallel contours. Along these diagonals, the models exhibit fairly similar line spectra. Thus, the stellar parameters derived for stars in the regime of parameter degeneracy solely depend on small differences of the synthetic

2 http://www.astro.physik. uni-potsdam.de/PoWR.html 
Table 2. Parameters of LMC WN stars.

\begin{tabular}{|c|c|c|c|c|c|c|c|c|c|c|c|c|c|c|c|}
\hline BAT99 & Subtype & Ref. & $\begin{array}{c}T_{*} \\
{[\mathrm{kK}]}\end{array}$ & $\begin{array}{c}\log R_{\mathrm{t}} \\
{\left[R_{\odot}\right]}\end{array}$ & $\begin{array}{c}v_{\infty} \\
{\left[\mathrm{km} \mathrm{s}^{-1}\right]}\end{array}$ & $\begin{array}{c}E_{b-v} \\
{[\mathrm{mag}]}\end{array}$ & $\begin{array}{c}M_{v} \\
{[\mathrm{mag}]}\end{array}$ & $\begin{array}{c}R_{*} \\
{\left[R_{\odot}\right]}\end{array}$ & $\begin{array}{c}\log \dot{M} \\
{\left[M_{\odot} / \mathrm{yr}\right]}\end{array}$ & $\begin{array}{l}\log L \\
{\left[L_{\odot}\right]}\end{array}$ & $\eta$ & $\begin{array}{c}M^{a} \\
{\left[M_{\odot}\right]}\end{array}$ & $X_{\mathrm{H}}$ & Bin. $^{b}$ & Ref. \\
\hline 001 & WN3b & 1 & 89 & 0.60 & 1600 & 0.14 & -3.32 & 1.9 & -5.18 & 5.30 & 2.6 & 12 & 0.0 & & \\
\hline 002 & WN2b(h) & 2 & 141 & 0.30 & 1600 & 0.13 & -2.36 & 0.8 & -5.28 & 5.37 & 1.8 & 13 & 0.0 & & \\
\hline 003 & WN4b & 1 & 79 & 0.60 & 1600 & 0.12 & -4.36 & 3.0 & -4.88 & 5.51 & 3.2 & 16 & 0.0 & & \\
\hline 005 & WN2b & 2 & 141 & 0.30 & 1600 & 0.27 & -2.94 & 0.9 & -5.22 & 5.45 & 1.7 & 15 & 0.0 & & \\
\hline 006 & $\mathrm{O} 3 \mathrm{f}^{*}+\mathrm{O}$ & 3 & 56 & 1.80 & 1600 & 0.08 & -6.59 & 17.7 & -5.52 & 6.45 & 0.1 & 94 & 0.2 & $\mathrm{x}$ & $4,3,5$ \\
\hline 007 & WN4b & 1 & 158 & -0.10 & 1600 & 0.08 & -5.02 & 1.1 & -4.48 & 5.84 & 3.8 & 25 & 0.0 & & \\
\hline 012 & O2 If*/WN5 & 6 & 50 & 1.70 & 2400 & 0.10 & -5.19 & 10.6 & -5.53 & 5.80 & 0.5 & 53 & 0.5 & $\mathrm{x}$ & 7 \\
\hline 013 & WN10 & 1 & 28 & 1.00 & 400 & 0.20 & -6.34 & 25.3 & -4.69 & 5.56 & 1.1 & 35 & 0.4 & & \\
\hline 014 & WN4o(+OB) & 2 & 67 & 1.15 & 1600 & 0.09 & -5.17 & 6.4 & -5.21 & 5.86 & 0.7 & 26 & 0.0 & ? & 2,8 \\
\hline 015 & WN4b & 1 & 89 & 0.50 & 1600 & 0.08 & -4.44 & 2.6 & -4.83 & 5.57 & 3.1 & 17 & 0.0 & & \\
\hline 016 & WN7h & 7 & 50 & 0.85 & 1000 & 0.09 & -6.12 & 10.6 & -4.64 & 5.80 & 1.8 & 42 & 0.3 & & \\
\hline 017 & WN4o & 1 & 67 & 0.90 & 1600 & 0.11 & -4.79 & 5.2 & -4.97 & 5.69 & 1.7 & 20 & 0.0 & & \\
\hline 018 & WN3(h) & 2 & 71 & 1.00 & 1600 & 0.10 & -4.31 & 4.4 & -5.24 & 5.63 & 1.1 & 29 & 0.2 & & \\
\hline 019 & WN4b+O5: & 2 & 79 & 0.75 & 1600 & 0.16 & -5.33 & 6.2 & -4.63 & 6.14 & 1.3 & 39 & 0.0 & $\mathrm{x}^{c}$ & 2 \\
\hline 021 & $\mathrm{WN} 4 \mathrm{o}(+\mathrm{OB})$ & 2 & 67 & 1.30 & 1600 & 0.09 & -5.76 & 10.6 & -5.11 & 6.30 & 0.3 & 51 & 0.0 & $?$ & 2,8 \\
\hline 022 & WN9h & 1 & 32 & 1.10 & 400 & 0.13 & -7.00 & 25.1 & -4.85 & 5.75 & 0.5 & 44 & 0.4 & & \\
\hline 023 & WN3(h) & 2 & 71 & 1.00 & 1600 & 0.60 & -3.98 & 4.0 & -5.30 & 5.55 & 1.1 & 17 & 0.0 & & \\
\hline 024 & WN4b & 1 & 100 & 0.30 & 2400 & 0.10 & -4.39 & 2.0 & -4.53 & 5.54 & 10.1 & 17 & 0.0 & & \\
\hline 025 & WN4ha & 2 & 67 & 1.30 & 1600 & 0.15 & -4.01 & 4.5 & -5.67 & 5.55 & 0.5 & 26 & 0.2 & & \\
\hline 026 & WN4b & 1 & 71 & 0.70 & 1600 & 0.14 & -4.38 & 4.3 & -4.79 & 5.62 & 3.0 & 18 & 0.0 & & \\
\hline 027 & WN5b(+B1 Ia) & 2 & 71 & 1.40 & 1000 & 0.23 & -8.22 & 29.8 & -4.79 & 7.30 & 0.0 & 587 & 0.2 & ? & 9 \\
\hline 029 & $\mathrm{WN} 4 \mathrm{~b}+\mathrm{OB}$ & 2 & 71 & 0.80 & 1600 & 0.12 & -4.37 & 3.7 & -5.03 & 5.50 & 2.3 & 16 & 0.0 & $\mathrm{x}$ & 2 \\
\hline 030 & WN6h & 1 & 47 & 1.10 & 1000 & 0.07 & -5.48 & 10.0 & -5.05 & 5.65 & 1.0 & 34 & 0.3 & & \\
\hline 031 & WN4b & 2 & 75 & 0.70 & 1600 & 0.17 & -3.81 & 2.7 & -5.09 & 5.33 & 3.0 & 12 & 0.0 & ? & 2 \\
\hline 032 & WN6(h) & 1 & 47 & 1.10 & 1600 & 0.08 & -6.14 & 13.9 & -4.63 & 5.94 & 2.1 & 44 & 0.2 & $\mathrm{x}$ & 7,10 \\
\hline 033 & O fpe/WN9? & 7 & 28 & 1.30 & 400 & 0.37 & -8.48 & 74.8 & -4.43 & 6.50 & 0.2 & 103 & 0.2 & & \\
\hline 035 & WN3(h) & 2 & 71 & 0.90 & 1600 & 0.11 & -4.11 & 4.2 & -5.11 & 5.60 & 1.5 & 24 & 0.1 & & \\
\hline 036 & $\mathrm{WN} 4 \mathrm{~b} / \mathrm{WCE}+\mathrm{OB}$ & 2 & 79 & 0.70 & 1600 & 0.13 & -4.33 & 3.8 & -4.88 & 5.71 & 2.0 & 21 & 0.0 & $?$ & 2,11 \\
\hline 037 & WN3o & 2 & 79 & 0.80 & 1600 & 0.50 & -4.12 & 3.5 & -5.07 & 5.65 & 1.5 & 19 & 0.0 & & \\
\hline 040 & WN4(h)a & 2 & 63 & 1.20 & 1600 & 0.15 & -4.41 & 5.4 & -5.39 & 5.62 & 0.8 & 29 & 0.2 & $?^{c}$ & \\
\hline 041 & $\mathrm{WN} 4 \mathrm{~b}$ & 1 & 100 & 0.40 & 1300 & 0.12 & -4.11 & 2.1 & -4.90 & 5.60 & 2.0 & 18 & 0.0 & & \\
\hline 042 & WN5b(h)(+B3 I) & 2 & 71 & 1.70 & 1000 & 0.30 & -9.88 & 66.6 & -4.71 & 8.00 & 0.0 & - & 0.4 & $?^{c}$ & $2,9,12$ \\
\hline 043 & $\mathrm{WN} 4 \mathrm{o}+\mathrm{OB}$ & 2 & 67 & 1.10 & 1600 & 0.13 & -4.84 & 6.3 & -5.15 & 5.85 & 0.8 & 25 & 0.0 & $\mathrm{x}$ & 2 \\
\hline 044 & WN8ha & 7 & 45 & 1.10 & 700 & 0.12 & -5.59 & 11.3 & -5.12 & 5.66 & 0.6 & 40 & 0.4 & & \\
\hline 046 & WN4o & 1 & 63 & 1.00 & 1600 & 0.21 & -4.09 & 4.4 & -5.23 & 5.44 & 1.7 & 14 & 0.0 & & \\
\hline 047 & WN3b & 2 & 89 & 0.60 & 1300 & 0.20 & -3.97 & 2.6 & -5.06 & 5.59 & 1.4 & 18 & 0.0 & $?^{c}$ & \\
\hline 048 & WN4b & 1 & 89 & 0.40 & 1600 & 0.10 & -4.22 & 2.1 & -4.81 & 5.40 & 4.9 & 14 & 0.0 & & \\
\hline 049 & WN4:b+O8V & 2 & 71 & 1.80 & 2400 & 0.15 & -5.49 & 9.9 & -5.73 & 6.34 & 0.1 & 122 & 0.6 & $\mathrm{x}$ & 2,13 \\
\hline 050 & WN5h & 14 & 56 & 1.40 & 1600 & 0.18 & -4.75 & 7.1 & -5.52 & 5.65 & 0.5 & 39 & 0.4 & & \\
\hline 051 & WN3b & 1 & 89 & 0.60 & 1600 & 0.02 & -3.39 & 1.9 & -5.18 & 5.30 & 2.6 & 12 & 0.0 & & \\
\hline 054 & WN8ha & 7 & 38 & 1.30 & 1000 & 0.50 & -6.23 & 17.7 & -4.97 & 5.75 & 0.9 & 34 & 0.2 & & \\
\hline 055 & WN11h & 1 & 28 & 1.40 & 400 & 0.13 & -7.04 & 32.3 & -5.13 & 5.77 & 0.2 & 45 & 0.4 & & \\
\hline 056 & WN4b & 1 & 71 & 0.75 & 1600 & 0.12 & -4.46 & 4.0 & -4.91 & 5.56 & 2.6 & 17 & 0.0 & & \\
\hline 057 & WN4b & 1 & 79 & 0.60 & 1600 & 0.10 & -4.04 & 2.7 & -4.96 & 5.40 & 3.4 & 14 & 0.0 & & \\
\hline 058 & WN7h & 7 & 47 & 1.15 & 1000 & 0.50 & -5.35 & 9.9 & -5.13 & 5.64 & 0.8 & 34 & 0.3 & & \\
\hline 059 & $\mathrm{WN} 4 \mathrm{~b}+\mathrm{O} 8 \mathrm{:}$ & 2 & 71 & 1.30 & 1600 & 0.16 & -6.01 & 11.2 & -5.07 & 6.45 & 0.2 & 66 & 0.0 & ? & 2 \\
\hline 060 & WN4(h)a & 2 & 63 & 1.40 & 2400 & 0.15 & -4.82 & 6.5 & -5.40 & 5.78 & 0.8 & 35 & 0.2 & & \\
\hline 062 & WN3(h) & 2 & 71 & 0.90 & 1600 & 0.12 & -3.85 & 3.4 & -5.25 & 5.41 & 1.7 & 19 & 0.1 & & \\
\hline 063 & WN4ha: & 2 & 63 & 1.20 & 1600 & 0.10 & -4.33 & 5.2 & -5.42 & 5.58 & 0.8 & 36 & 0.4 & & \\
\hline 064 & WN4o+O9: & 2 & 71 & 1.10 & 1600 & 0.26 & -5.18 & 7.1 & -5.07 & 6.05 & 0.6 & 34 & 0.0 & $\mathrm{x}$ & 2 \\
\hline 065 & WN4o & 2 & 67 & 0.90 & 1600 & 0.45 & -4.84 & 5.6 & -4.92 & 5.75 & 1.7 & 22 & 0.0 & & \\
\hline 066 & WN3(h) & 2 & 89 & 1.00 & 1600 & 0.13 & -3.73 & 3.3 & -5.42 & 5.78 & 0.5 & 35 & 0.2 & & \\
\hline 067 & WN5ha & 2 & 47 & 1.30 & 1600 & 0.33 & -6.11 & 14.3 & -4.91 & 5.96 & 1.1 & 51 & 0.3 & $?^{c}$ & \\
\hline 068 & O3.5 If*/WN7 & 6 & 45 & 1.60 & 1000 & 0.52 & -6.22 & 16.7 & -5.46 & 6.00 & 0.2 & 76 & 0.6 & & \\
\hline 071 & WN4+O8: & 2 & 63 & 1.30 & 1600 & 0.38 & -5.16 & 8.2 & -5.27 & 5.98 & 0.4 & 31 & 0.0 & $\mathrm{x}$ & 2 \\
\hline 072 & $\mathrm{WN} 4 \mathrm{~h}+\mathrm{O} 3:$ & 2 & 71 & 1.40 & 1600 & 0.40 & -4.31 & 5.3 & -5.71 & 5.80 & 0.2 & 47 & 0.4 & $?$ & 2 \\
\hline 073 & WN5ha & 14 & 60 & 1.40 & 1600 & 0.20 & -4.67 & 6.8 & -5.54 & 5.72 & 0.4 & 43 & 0.4 & & \\
\hline
\end{tabular}

Notes. ${ }^{(a)}$ Masses calculated from the luminosity, using the mass-luminosity relation derived by Gräfener et al. $(2011),{ }^{(b)} \mathrm{x}=$ detected, ? $=$ questionable, ${ }^{(c)}$ high X-ray emission.

References. (1) BAT99; (2) Foellmi et al. (2003b); (3) Niemela et al. (2001); (4) Niemela et al. (1995); (5) Koenigsberger et al. (2003); (6) Crowther \& Walborn (2011); (7) Schnurr et al. (2008); (8) Breysacher (1981); (9) Smith et al. (1996,and references therein); (10) Moffat (1989); (11) Crowther et al. (1995b); (12) Seggewiss et al. (1991); (13) Niemela (1991); (14) Crowther \& Hadfield (2006); (15) Doran et al. (2013); (16) Evans et al. (2011); (17) Taylor et al. (2011); (18) Schnurr et al. (2009a); (19) Sana et al. (2013b); (20) Foellmi et al. (2006). 
Table 2. continued.

\begin{tabular}{|c|c|c|c|c|c|c|c|c|c|c|c|c|c|c|c|}
\hline BAT99 & Subtype & Ref. & $\begin{array}{c}T_{*} \\
{[\mathrm{kK}]}\end{array}$ & $\begin{array}{c}\log R_{\mathrm{t}} \\
{\left[R_{\odot}\right]}\end{array}$ & $\begin{array}{c}v_{\infty} \\
{\left[\mathrm{km} \mathrm{s}^{-1}\right]}\end{array}$ & $\begin{array}{c}E_{b-v} \\
{[\mathrm{mag}]}\end{array}$ & $\begin{array}{c}M_{v} \\
{[\mathrm{mag}]}\end{array}$ & $\begin{array}{c}R_{*} \\
{\left[R_{\odot}\right]}\end{array}$ & $\begin{array}{c}\log \dot{M} \\
{\left[M_{\odot} / \mathrm{yr}\right]}\end{array}$ & $\begin{array}{c}\log L \\
{\left[L_{\odot}\right]}\end{array}$ & $\eta$ & $\begin{array}{c}M^{a} \\
{\left[M_{\odot}\right]}\end{array}$ & $X_{\mathrm{H}}$ & Bin. $^{b}$ & Ref. \\
\hline 074 & WN3(h)a & 2 & 79 & 1.25 & 2000 & 0.20 & -3.82 & 3.7 & -5.62 & 5.69 & 0.5 & 32 & 0.2 & & \\
\hline 075 & WN4o & 2 & 71 & 0.80 & 1600 & 0.07 & -4.32 & 4.0 & -4.99 & 5.56 & 2.2 & 17 & 0.0 & & \\
\hline 076 & WN9ha & 7 & 35 & 1.10 & 400 & 0.26 & -6.31 & 17.9 & -5.07 & 5.66 & 0.4 & 30 & 0.2 & & \\
\hline 077 & WN7ha & 7 & 45 & 1.60 & 1000 & 0.27 & -5.18 & 41.6 & -4.87 & 6.79 & 0.1 & 305 & 0.7 & $\mathrm{x}^{c}$ & 7,10 \\
\hline 078 & WN6(+O8 V) & 2 & 71 & 0.85 & 1600 & 0.20 & -4.48 & 4.7 & -4.96 & 5.70 & 1.7 & 32 & 0.2 & $?^{c}$ & \\
\hline 079 & WN7ha+OB & 7 & 42 & 1.20 & 1600 & 0.50 & -7.03 & 22.8 & -4.46 & 6.17 & 1.9 & 61 & 0.2 & $?^{c}$ & \\
\hline 080 & WN5h:a & 7 & 45 & 1.70 & 2400 & 0.50 & -7.31 & 26.5 & -4.93 & 6.40 & 0.5 & 87 & 0.2 & $?^{c}$ & \\
\hline 081 & WN5h & 2 & 47 & 1.35 & 1000 & 0.33 & -4.47 & 8.2 & -5.55 & 5.48 & 0.5 & 32 & 0.4 & & \\
\hline 082 & WN3b & 1 & 100 & 0.60 & 1600 & 0.27 & -3.68 & 1.9 & -5.16 & 5.53 & 1.6 & 16 & 0.0 & $?^{c}$ & \\
\hline 086 & WN3(h) & 15 & 71 & 1.00 & 1600 & 0.36 & -3.37 & 3.1 & -5.46 & 5.33 & 1.3 & 12 & 0.0 & & \\
\hline 088 & WN4b/WCE & 2 & 112 & 0.40 & 1600 & 0.84 & -4.19 & 2.1 & -4.81 & 5.80 & 1.9 & 24 & 0.0 & & \\
\hline 089 & WN7h & 1 & 50 & 0.90 & 1000 & 0.28 & -5.37 & 10.3 & -4.73 & 5.78 & 1.5 & 35 & 0.2 & & \\
\hline 091 & WN6(h) & 16 & 50 & 1.00 & 1000 & 0.33 & -5.87 & 6.8 & -5.15 & 5.42 & 1.3 & 23 & 0.2 & & \\
\hline 092 & $\mathrm{WN} 3: \mathrm{b}(+\mathrm{O})+\mathrm{B} 1 \mathrm{Ia}$ & 7 & 45 & 1.50 & 1000 & 0.39 & -8.69 & 50.0 & -4.60 & 6.95 & 0.1 & 240 & 0.2 & $\mathrm{x}^{c}$ & 7,10 \\
\hline 093 & O3 If* & 6,16 & 45 & 1.80 & 1600 & 0.24 & -5.65 & 14.9 & -5.63 & 5.90 & 0.2 & 67 & 0.6 & $?^{c}$ & \\
\hline 094 & WN4b & 1 & 141 & 0.00 & 1600 & 0.29 & -4.80 & 1.3 & -4.51 & 5.80 & 3.9 & 24 & 0.0 & & \\
\hline 095 & $\mathrm{WN} 7 \mathrm{~h}+\mathrm{OB}$ & 16 & 50 & 0.80 & 1600 & 0.25 & -6.36 & 13.3 & -4.21 & 6.00 & 4.9 & 48 & 0.2 & $\mathrm{x}$ & 7 \\
\hline 096 & WN8 & 7 & 42 & 1.10 & 1000 & 0.70 & -7.55 & 28.1 & -4.37 & 6.35 & 0.9 & 80 & 0.2 & & \\
\hline 097 & O3.5 If*/WN7 & 6,16 & 45 & 1.70 & 1600 & 0.60 & -7.19 & 23.7 & -5.18 & 6.30 & 0.3 & 115 & 0.6 & & \\
\hline 098 & WN6 & 7 & 45 & 1.40 & 1600 & 0.80 & -8.11 & 37.5 & -4.43 & 6.70 & 0.6 & 226 & 0.6 & & \\
\hline 099 & O2.5 If*/WN6 & 6,16 & 45 & 1.80 & 1600 & 0.30 & -6.77 & 14.9 & -5.63 & 5.90 & 0.2 & 42 & 0.2 & $\mathrm{x}^{c}$ & 7 \\
\hline 100 & WN7 & 7 & 47 & 1.00 & 1000 & 0.28 & -6.80 & 17.7 & -4.52 & 6.15 & 1.0 & 59 & 0.2 & $?^{c}$ & \\
\hline 102 & WN6 & 7 & 45 & 1.30 & 1600 & 0.70 & -8.38 & 42.1 & -4.21 & 6.80 & 0.8 & 221 & 0.4 & $?^{c}$ & 7 \\
\hline 103 & $\mathrm{WN} 5(\mathrm{~h})+\mathrm{O}$ & 16 & 47 & 1.30 & 1600 & 0.40 & -7.13 & 19.9 & -4.70 & 6.25 & 0.9 & 87 & 0.4 & $\mathrm{x}^{c}$ & 7,10 \\
\hline 104 & O2 If*/WN5 & 6 & 63 & 1.50 & 2400 & 0.38 & -5.48 & 9.0 & -5.34 & 6.06 & 0.5 & 66 & 0.4 & & \\
\hline 105 & O2 If* & 6 & 50 & 1.80 & 1600 & 0.30 & -6.93 & 21.1 & -5.41 & 6.40 & 0.1 & 134 & 0.6 & $?^{c}$ & \\
\hline 106 & WN5h & 1 & 56 & 1.30 & 2400 & 0.35 & -6.86 & 19.0 & -4.55 & 6.51 & 1.0 & 130 & 0.4 & & \\
\hline 107 & O6.5 Iafc+O6 Iaf & 17 & 35 & 1.50 & 1000 & 0.26 & -7.45 & 37.9 & -4.78 & 6.31 & 0.4 & 95 & 0.4 & $\mathrm{x}^{c}$ & 10,17 \\
\hline 108 & WN5h & 1 & 56 & 1.40 & 2400 & 0.37 & -7.10 & 28.8 & -4.43 & 6.87 & 0.6 & 256 & 0.4 & & \\
\hline 109 & WN5h & 1 & 56 & 1.40 & 2400 & 0.39 & -6.50 & 23.4 & -4.56 & 6.69 & 0.7 & 179 & 0.4 & & \\
\hline 110 & O2 If* & 6 & 50 & 1.70 & 2400 & 0.41 & -6.36 & 17.1 & -5.22 & 6.22 & 0.4 & 113 & 0.7 & & \\
\hline 111 & WN9ha & 1 & 45 & 1.70 & 1000 & 0.43 & -7.00 & 22.3 & -5.42 & 6.25 & 0.1 & 118 & 0.7 & $?^{c}$ & \\
\hline 112 & WN5h & 1 & 56 & 1.30 & 2400 & 0.44 & -7.20 & 18.4 & -4.57 & 6.48 & 1.0 & 99 & 0.2 & $?^{c}$ & 18 \\
\hline 113 & O2 If*/WN5 & 6,16 & 50 & 1.70 & 1600 & 0.28 & -6.08 & 14.8 & -5.49 & 6.09 & 0.2 & 54 & 0.2 & $\mathrm{x}^{c}$ & 7 \\
\hline 114 & O2 If*/WN5 & 6,16 & 63 & 1.70 & 2400 & 0.31 & -6.18 & 13.9 & -5.35 & 6.44 & 0.2 & 116 & 0.4 & $?^{c}$ & \\
\hline 116 & WN5h:a & 7 & 63 & 1.30 & 2400 & 0.75 & -7.93 & 28.1 & -4.29 & 7.05 & 0.5 & 390 & 0.4 & $?^{c}$ & 7 \\
\hline 117 & WN5ha & 2 & 63 & 1.40 & 2400 & 0.19 & -6.33 & 13.3 & -4.93 & 6.40 & 0.5 & 109 & 0.4 & & \\
\hline 118 & WN6h & 1 & 47 & 1.10 & 1600 & 0.16 & -7.96 & 31.9 & -4.09 & 6.66 & 1.4 & 136 & 0.2 & $\mathrm{x}^{c}$ & 7,19 \\
\hline 119 & WN6h+? & 1 & 47 & 1.20 & 1600 & 0.29 & -7.64 & 28.8 & -4.31 & 6.57 & 1.0 & 116 & 0.2 & $\mathrm{x}^{c}$ & 7,10 \\
\hline 120 & WN9h & 1 & 32 & 1.40 & 500 & 0.15 & -6.53 & 20.6 & -5.33 & 5.58 & 0.3 & 32 & 0.3 & & \\
\hline 122 & WN5h & 2 & 50 & 1.15 & 1600 & 0.28 & -6.90 & 17.3 & -4.56 & 6.23 & 1.3 & 67 & 0.2 & & \\
\hline 124 & WN4 & 2 & 63 & 1.10 & 1600 & 0.30 & -4.32 & 4.5 & -5.37 & 5.45 & 1.2 & 15 & 0.0 & & \\
\hline 126 & WN4b+O8: & 2 & 71 & 1.10 & 1600 & 0.22 & -6.05 & 11.1 & -4.78 & 6.44 & 0.5 & 65 & 0.0 & $?^{c}$ & 2 \\
\hline 128 & WN3b & 2 & 112 & 0.30 & 1600 & 0.17 & -3.74 & 1.4 & -4.93 & 5.44 & 3.4 & 14 & 0.0 & & \\
\hline 129 & WN3(h)a+O5 V & 20 & 79 & 1.25 & 2000 & 0.35 & -5.01 & 6.7 & -5.24 & 6.20 & 0.4 & 64 & 0.2 & $\mathrm{x}$ & 7 \\
\hline 130 & WN11h & 1 & 28 & 1.30 & 200 & 0.25 & -6.70 & 29.1 & -5.35 & 5.68 & 0.1 & 41 & 0.4 & & \\
\hline 131 & WN4b & 2 & 71 & 0.75 & 1600 & 0.13 & -4.76 & 4.6 & -4.83 & 5.67 & 2.5 & 20 & 0.0 & & \\
\hline 132 & WN4b(h) & 2 & 79 & 0.50 & 1600 & 0.23 & -4.82 & 3.3 & -4.67 & 5.58 & 4.4 & 17 & 0.0 & & \\
\hline 133 & WN11h & 1 & 28 & 1.35 & 200 & 0.11 & -6.85 & 29.4 & -5.42 & 5.69 & 0.1 & 41 & 0.4 & & \\
\hline 134 & WN4b & 1 & 79 & 0.60 & 1600 & 0.06 & -4.24 & 3.0 & -4.88 & 5.51 & 3.2 & 16 & 0.0 & & \\
\hline
\end{tabular}

spectra. There are indeed some stars in our sample that fall into this regime of parameter degeneracy (see Appendix B).

For 27 stars, we preselect suitable grid models by means of a $\chi_{v}^{2}$-fitting technique. This method is based on a reduced $\chi_{v}^{2}$-statistic, which is calculated for the rectified observations with respect to the model spectra in our grids. For details of this fitting technique, we refer to Todt et al. (2013).

\subsection{Spectral fitting}

After preliminary parameters have been estimated either by the $\chi_{v}^{2}$-fit or by the contour plots, we compare observations and models in detail for each star. A typical fit of the normalized line spectrum is shown in Fig. 3, while analogous plots for each star of the sample can be found in Appendix C.

While most of the observed spectra are given in normalized form, some of the spectra (from IUE, HST, and Torres-Dodgen \& Massey 1988) are flux-calibrated. These data are normalized consistently through division by the reddened model continuum. In this respect, spectral fitting is an iterative process, coupled with the fitting of the spectral energy distribution described below.

With the starting estimates for $T_{*}$ and $R_{\mathrm{t}}$, we carefully compare the observed line spectrum with models of neighboring parameters, and finally determine the best fitting values. The 

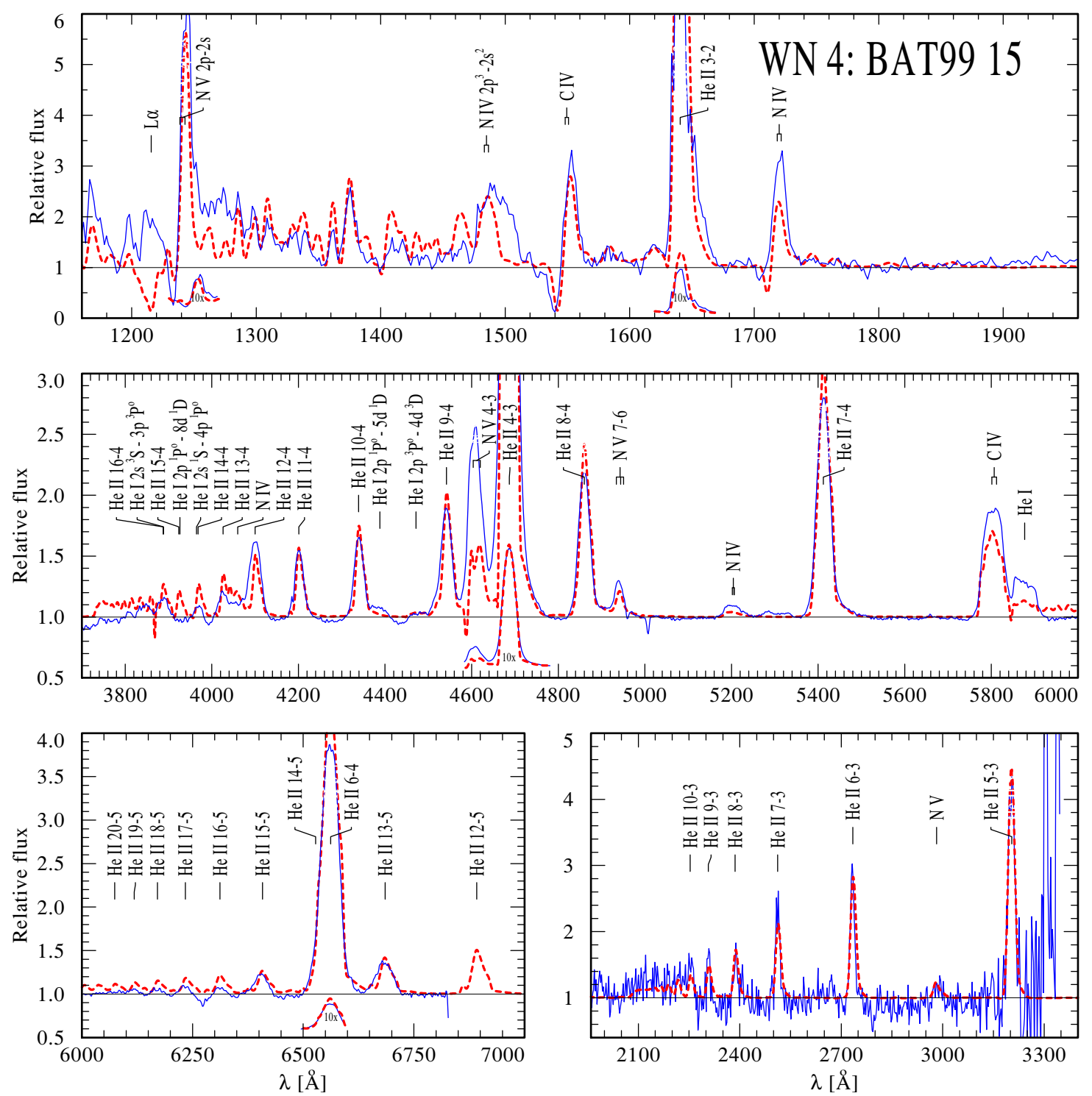

Fig. 3. Normalized spectrum of the WNE star BAT99015. The thin (blue) solid line depicts the observation, whereas the thick (red) dashed line represents the synthetic spectrum of the best fitting PoWR model.

uncertainty is usually smaller than one grid cell, i.e., the error margins are smaller than \pm 0.05 dex in $T_{*}$ and \pm 0.1 dex in $R_{\mathrm{t}}$. The latter translates to an uncertainty of \pm 0.15 dex for the mass-loss rate (cf. Eq. (2)). This, of course, does not account for systematic errors, because, for instance the model assumptions are not exactly fulfilled.

The terminal wind velocity, $v_{\infty}$, mainly influences the width of the line profiles. For 62 of our program stars, Niedzielski \& Skorzynski (2002) and Niedzielski et al. (2004) measured the wind velocities from P-Cygni profiles in the UV. Depending on the considered line, they obtain quite different values for the same star. Two possible reasons are: (a) while the $\beta$-law for the velocity field quickly approaches the terminal velocity, the winds are in fact further accelerated even at large distances from the star. Therefore, the strongest lines give the highest wind velocity. (b) The velocity field in the wind has some nonuniform, stochastic component that is not perfectly described by our assumption of a constant and isotropic microturbulence. In any case, the largest of the velocities given by Niedzielski et al. (2004) often yield optical emission line profiles that are considerably broader than observed.
Therefore, we prefer to perform our own estimates of $v_{\infty}$ from the width of the optical emission lines. First, we inspect whether the standard $v_{\infty}$ of the respective grid is sufficient to reproduce the observed line width. If not, we recalculate the model for the considered star with a more appropriate estimate, aiming at an accuracy of about $\pm 200 \mathrm{~km} \mathrm{~s}^{-1}$. The values of $v_{\infty}$ used for the final fits and the subsequent discussion are compiled in Table 2.

Among the studied sample, we found a couple of stars (especially the putatively single WNE stars BAT997, 51, 88, and 94) to exhibit very unique spectra. Their emission lines have a round shape that is distinctly different from those of all other stars, but similar to the shape of WR 2 in the Milky Way (HGL06). Such profiles can be reproduced by convolving the model spectrum with a rotation profile of very high $v \sin i$. A more adequate treatment of rotational broadening in WR winds presently confirmed that rotation might in principle account for these spectra (Shenar et al. 2014).

A further important model parameter is the hydrogen abundance. Its determination is one of the major aims of this paper. For this purpose, we calculated three extended model grids for 


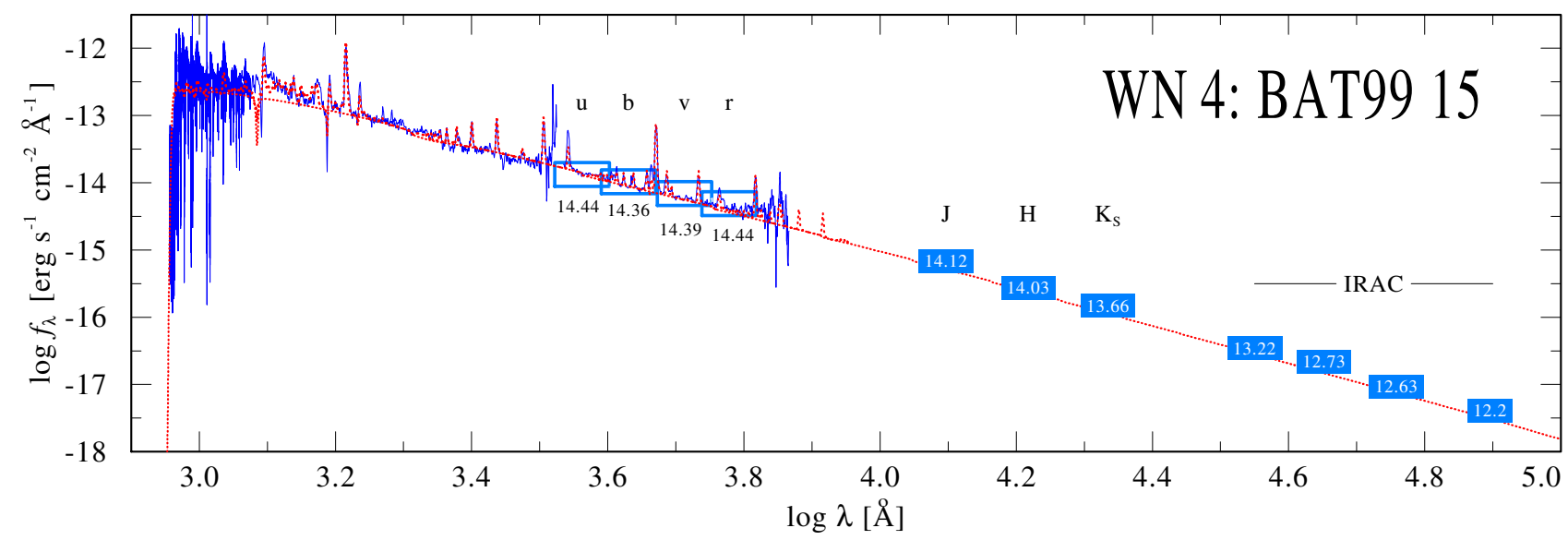

Fig. 4. Spectral energy distribution of the WNE star BAT99 015. Flux-calibrated FUSE, IUE, and CTIO spectra (blue noisy line) and multiband photometry (blue boxes, labeled with the magnitudes) are compared to the model flux (red dotted lines), accounting for interstellar extinction.

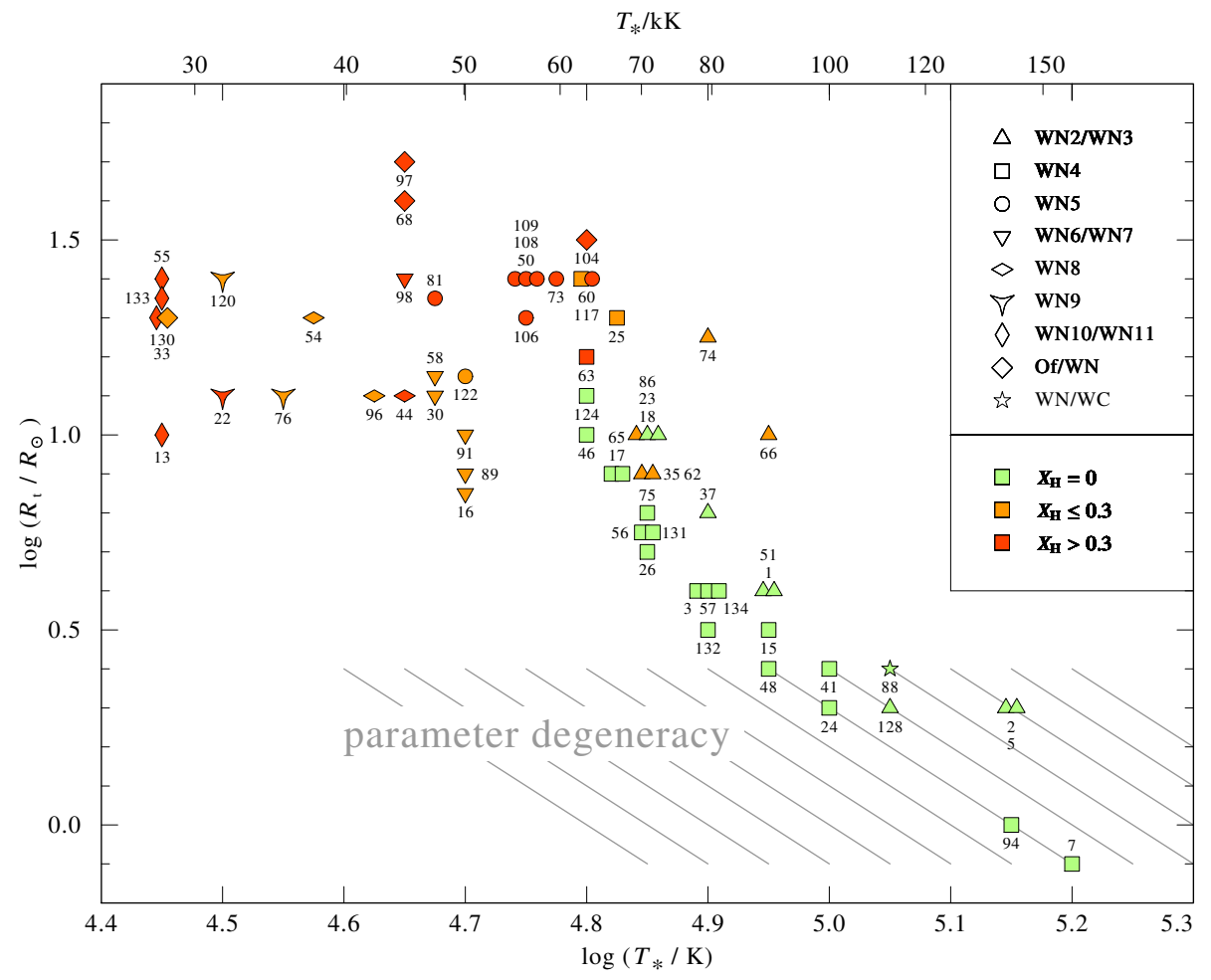

Fig. 5. Positions of the analyzed WN stars in the $\log T_{*}-\log R_{\mathrm{t}}$-plane. The labels refer to the BAT99 catalog. Different WN subtypes are distinguished by the shape of the symbols, as shown in the inlet. The hydrogen abundance is color-coded in three steps (undetectable, about 0.2 , and about 0.4 mass fraction). The lower hatched part roughly indicates the region where the parameter space becomes degenerate because of large optical thickness of the wind. In this part, the stars can be shifted parallel to the gray lines without significant changes in the synthetic normalized emission line spectrum. hydrogen mass fractions of $0,0.2$, and 0.4 , respectively, plus a couple of models for 0.6. By comparison and tentative interpolation between these grids, we can estimate the hydrogen mass fraction with \pm 0.1 accuracy.

After the appropriate model has been selected from the line fit, it must be scaled to the correct luminosity by fitting the spectral energy distribution (SED) to the photometric and flux-calibrated observations (for example, see Fig. 4). The scaling corresponds to a simple vertical shift in this logarithmic plot, while the normalized emission line spectrum does not change between models with same transformed radius (cf. Sect. 3). The model flux is geometrically diluted according to the LMC distance modulus of 18.5 mag (Madore \& Freedman 1998; Pietrzyński et al. 2013), corresponding to a distance of $50 \mathrm{kpc}$.

The color excess $E_{b-v}$ must be adjusted simultaneously. The reddening encompasses contributions from both the internal LMC reddening and Galactic foreground reddening, assuming the Seaton reddening law (Seaton 1979) with $E_{b-v}=0.03 \mathrm{mag}$ for the latter. The remaining LMC excess is determined by adjusting the free $E_{b-v}$ parameter of the LMC reddening law determined by Howarth (1983).

Since the stellar flux in the optical and IR depends roughly linearly on the stellar temperature (Rayleigh-Jeans domain), the error in $T_{*}( \pm 0.05$ dex, see above $)$ influences the luminosity estimate directly. Additional uncertainties are inferred from the reddening correction, which is relatively small for our LMC stars, the imperfect SED fit, and the error margins of the photometry. These errors combine to a final accuracy of about \pm 0.1 dex in $\log \left(L / L_{\odot}\right)$ for those stars where photometry and flux-calibrated spectra are available. If only photometry is accessible, the accuracy is reduced to \pm 0.2 dex, due to a larger uncertainty in the SED fit.

\section{Results}

\subsection{Stellar parameters}

The analysis of the line spectrum yields the stellar temperature and the "transformed radius" as an immediate result from the 
PoWR models (cf. Sect. 4). The obtained values are compiled in Table 2 for all stars. Note that the parameters obtained for the binaries and binary candidates in our sample are biased, since we analyzed the spectra as if they were from a single star. A detailed analysis that accounts for the composite nature of these spectra is planned for a forthcoming paper.

The location of the WN stars in the $\log T_{*}-\log R_{\mathrm{t}}$-plane is shown in Fig. 5, omitting the binaries (even the questionable ones). The two parameters $\left(\log T_{*}\right.$ and $\left.\log R_{\mathrm{t}}\right)$ appear well correlated, although there is some scatter that exceeds their error margins. Compared to the corresponding diagram for the Galactic WN stars (cf. Fig. 2 in HGL06), the correlation is similar, while the LMC stars cover wider range of spectral subtypes at the cool end (WN10-11).

The WN atmospheres are dominated by helium, while hydrogen is generally depleted. The precise determination of the hydrogen abundance is delicate, because all $\mathrm{H}$ lines are blended with He II lines due to the wind broadening. Among the assumably 63 single WN stars of our sample, we find 27 stars where hydrogen is below detectability (see Table 2). The detection limit depends on the individual circumstances such as stellar parameters, quality of the observation, and consistency of the fit. Based on our experience, we estimate that hydrogen abundances higher than 0.05 (mass fraction) do not escape detection.

Line contributions from hydrogen can be found in the spectra of 36 putatively single WN stars, including the four Of/WN stars. There are about equal numbers of stars that fall into the 0.2 and the 0.4 category, respectively.

Overall, Fig. 5 shows a clear dichotomy regarding the hydrogen abundance. Hydrogen is typically undetectable in the hotter stars (early subtypes, WNE), while in the cooler stars (late subtypes, WNL) hydrogen is clearly present, albeit depleted. This pattern is similar to the one found for the Galactic WN stars (HGL06). Even the rough equality between the numbers of WN stars with and without hydrogen is similar to the Galactic sample.

Based on the obtained luminosity, we estimate the current stellar mass using the mass-luminosity relations for chemically homogeneous stars from Gräfener et al. (2011). For stars with hydrogen at their surface, we use their Eq. (9) for core H-burning stars, and for stars without hydrogen we use Eq. (10) for core He-burning stars. Among the putatively single stars, eight stars (BAT99 33, 97, 98, 106, 108, 109, 110, 117) exhibit current stellar masses in excess of $100 M_{\odot}$ and thus belong to the category of very massive stars (Vink et al. 2013).

In recent years, evidence is growing that canonical upper mass limit $\left(150 M_{\odot}\right)$ is exceeded (e.g., Crowther et al. 2010; Vink et al. 2013). In their study of the stellar population in the core of R136, Crowther et al. (2010) argued in favor of an upper mass limit that is roughly two times the canonical value. Although we achieve slightly lower values in our new analysis, we can confirm the range of stellar masses derived by these authors.

In addition to the stellar parameters listed in Table 2, we have compiled in Table A.3 the number of hydrogen and helium ionizing photons for each star as well as the corresponding Zanstra temperatures. These values have been derived from the ionizing flux of the best fitting model. In some cases however, the stellar wind is so opaque that only an insignificant number of ionizing photons can escape.

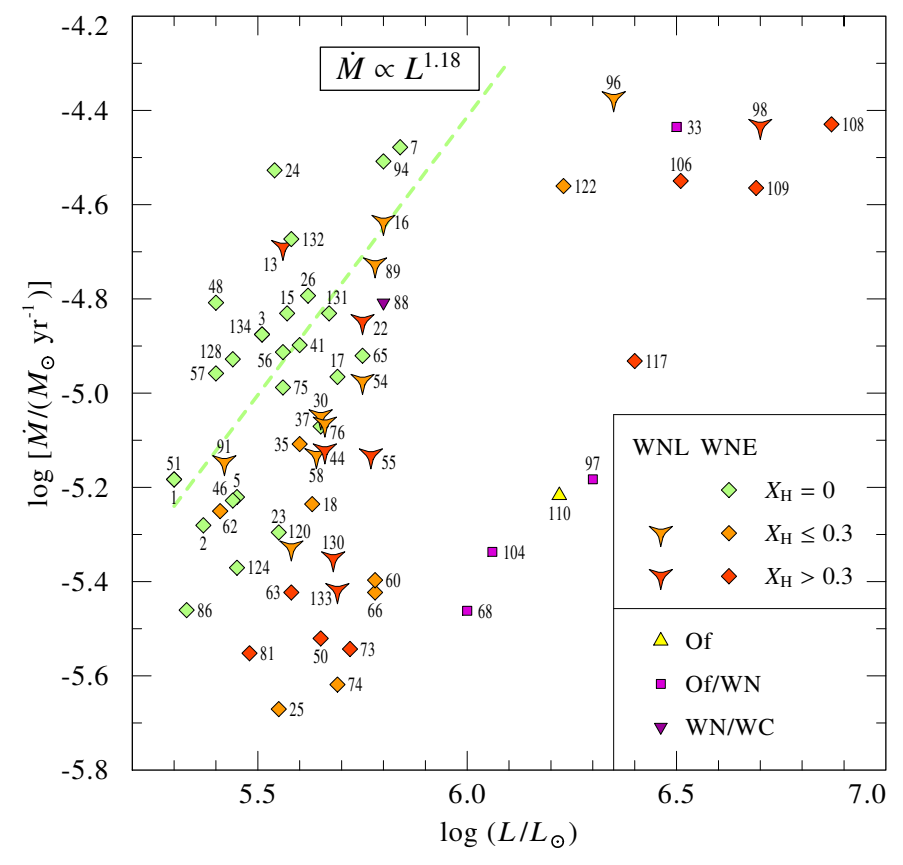

Fig. 6. Mass-loss rates versus luminosity for the putatively single WN stars. The symbol shapes refer to the WNE and WNL subclass, respectively. The atmospheric hydrogen mass fraction is color-coded, as indicated in the inlet. Also shown is a fit (green dashed line) to the hydrogen-free WNE stars.

\subsection{Mass-loss rates}

Mass loss of massive stars, especially WR stars, is of key importance for understanding their evolution and their influence on their environment. The huge injection of nuclear-enriched material is one of the main drivers of the chemical evolution of their host clusters and galaxies. For the evolution of WR stars, mass loss can be more important than nuclear fuel consumption.

The mass-loss rates obtained from our analyses are given in Table 2. One must keep in mind that the empirical $\dot{M}$ scales with the square-root of the clumping contrast, $\sqrt{D}$. The value $D=10$ is not accurately constrained, and may in fact vary from star to star or as function of radius (Nugis et al. 1998; Puls et al. 2006; Liermann \& Hamann 2008).

In Fig. 6, we plot the inferred mass-loss rates versus the luminosities of our sample stars. There is no simple correlation, but there is a characteristic pattern. The very luminous stars $\left(\log \left(L / L_{\odot}\right)>6.0\right)$, which all show atmospheric hydrogen, have the highest mass-loss rates $\left(\log \left(\dot{M} /\left(M_{\odot} / \mathrm{yr}\right)\right) \approx-4.5\right)$. These stars may be very massive stars directly evolving off the main sequence, possibly still burning hydrogen. However, there are also three Of/WN-type stars with luminosities $\geq 6$.0 dex that have weaker winds.

The bulk of "proper" WN stars populate the luminosity range from $\log \left(L / L_{\odot}\right)=5.3$ to 5.8 . Their mass-loss rates scatter over more than one order of magnitude (from -5.7 dex to -4.5 dex), but in clear correlation with the hydrogen abundance. The hydrogen-free stars, which are obviously helium burners, exhibit the strongest winds. Their $\log \dot{M}$ can be fitted to a linear relation with $\log L$, giving

$\dot{M}=\left(\frac{L}{10^{6} L_{\odot}}\right)^{1.18} 10^{-4.42} M_{\odot} / \mathrm{yr}$.

In the luminosity range below $5.9 \mathrm{dex}$, the mass-loss rates of those WN stars, which show a detectable amount of atmospheric 


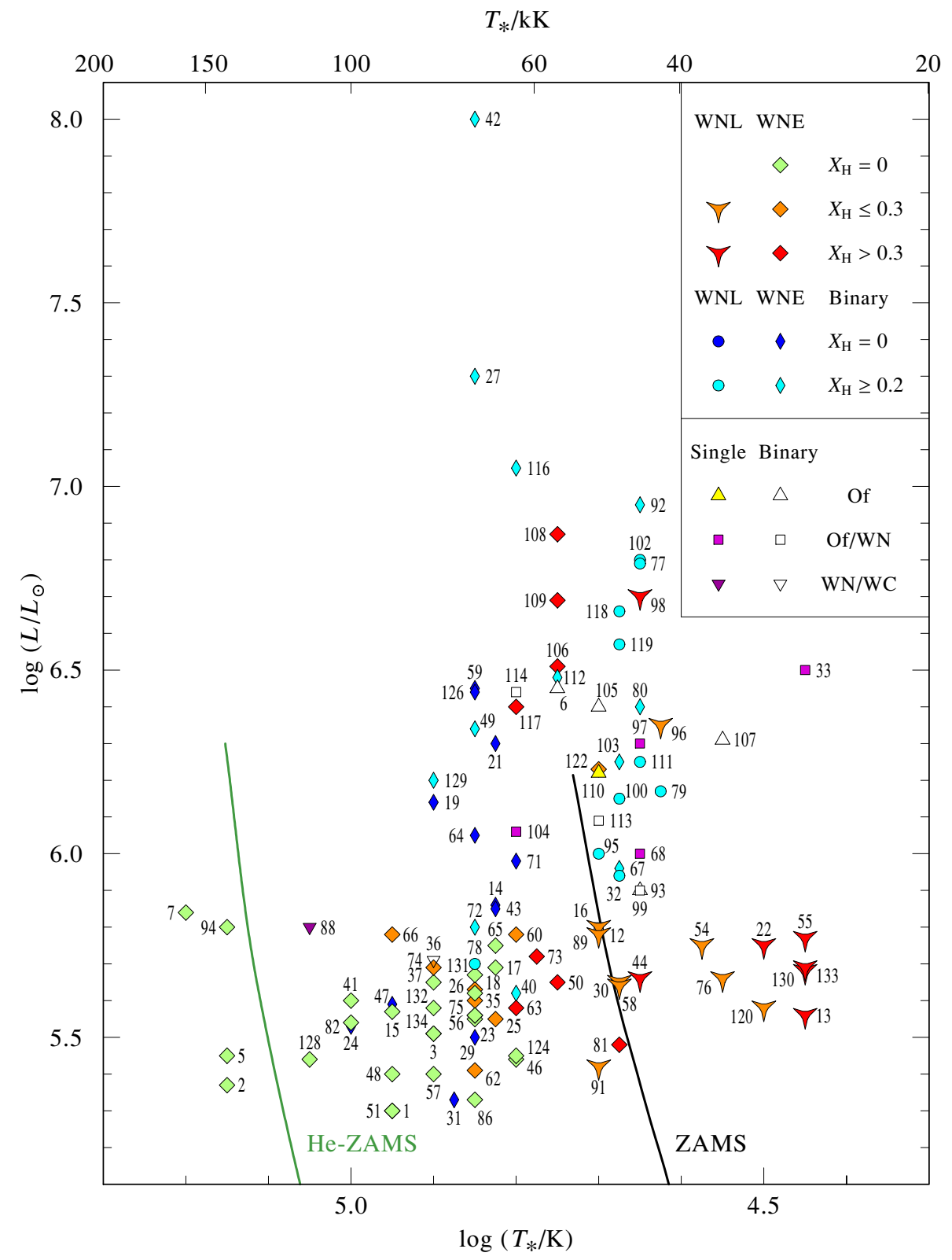

Fig. 7. HRD of our sample of LMC stars. As explained in the inlet, different symbols represent WNL and WNE stars, respectively. Binaries are also included, but distinguished by different symbols; their spectra have been analyzed as if they were single stars. The five O-type stars in our sample are plotted with their own symbols. Among the WN stars, the color codes the hydrogen mass fraction as defined in the inlet. The zero-age main sequences (ZAMS) for hydrogen-rich and pure-helium stars are shown for orientation. hydrogen, scatter a significantly. Nevertheless, it seems that the "proper" WN stars increase their mass-loss rates while evolving toward the Eddington limit, in line with hydrodynamical models calculated by Gräfener \& Hamann (2008).

Table 2 also gives the wind efficiency $\eta$, defined as the ratio between the rates of the wind momentum, $\dot{M} v_{\infty}$, and of the momentum of the radiation field, $L / c$ :

$\eta:=\frac{\dot{M} v_{\infty} c}{L}$

Wind efficiencies exceeding unity (the "single scattering limit") imply that an average photon undergoes multiple scatterings in the wind. Only specific hydrodynamic wind models can account for this effect. Gräfener \& Hamann (2005) obtained $\eta=2.5$ for a model of the Galactic WC star WR 111. More adequate for our sample, Gräfener \& Hamann (2008) calculated WN models for different metallicities and found that, under LMC conditions, the wind efficiency hardly exceeds unity. The empirical wind efficiencies obtained from our analysis are also moderate; the average values (only for the single stars) are 0.8 for the WNL and 2.1 for the WNE subtypes, respectively.

The mass-loss rates of the WN stars in the LMC obtained in the present study can be compared with those of the Galactic WN stars from Hamann et al. (2006). Note that different values for the clumping contrast have been adopted in these studies (LMC: $D=10$, MW: $D=4$ ). Since the empirical $\dot{M}$ depends on the degree of clumping, one must assume for such a comparison that the clumping properties do not differ between the LMC and MW, and scale the mass-loss rates according to Eq. (2).

The comparison reveals that the WN stars in the LMC have on average lower mass-loss rates by roughly a factor of two compared to their Galactic counterparts. This is consistent with a dependence of $\dot{M}$ with metallicity $Z$ to the power $0.9 \pm 0.3$, depending on the metallicity assumed for the LMC and the MW. This agrees well with the results obtained by Crowther (2006) as well as the exponent 0.86 theoretically derived by Vink \& de Koter (2005) for late-type WR stars. A detailed investigation of the mass-loss rate as a function of the metallicity will be the 
subject of a forthcoming paper, where we will incorporate the results from our study of the WN stars in the SMC.

\subsection{The Hertzsprung-Russell diagram}

The Hertzsprung-Russell diagram (HRD, Fig. 7) shows all stars analyzed in this paper. Notably, the diagram includes those stars that are already known to be binaries, but were analyzed here as if they were single stars. The highest luminosities in the HRD refer to such multiple stars.

In a second version of the HRD (Fig. 8), we restrict the sample to the WN single stars (including the Of/WN types). While the LMC stars are represented by color-filled symbols, the open symbols in the background are the Galactic WN stars analyzed by HGL06, Martins et al. (2008), Liermann et al. (2010), and Barniske et al. (2008).

One of the striking features in the HRD is the occurrence of a few extremely luminous stars. All these stars show atmospheric hydrogen. In the Galactic sample, there is also a detached group of very luminous stars, but the most luminous WN star encountered in the Galaxy - the "Peony star" WR 102ka (Barniske et al. 2008), reaches only about 6.5 dex solar luminosities.

The very luminous WN stars in the LMC are mostly of early subtypes (WNE) or Of/WN, while the Galactic ones are WNL types. Moreover, the number of WN stars in the highluminosity domain seems to be much larger in the Galactic sample. This might actually indicate a problem with the Galactic analyses that arises from the uncertainty of the stellar distances. Many of these Galactic WNL stars were "brightness calibrated" by means of those few WNL representatives that belong to clusters or associations. However, these young WNL stars may be exceptionally luminous. By employing them for the brightness calibration, the luminosities of other Galactic WNL stars might have been overestimated. Due to the known distance, the LMC results are free from such uncertainties.

Based on their known, uniform distance, we can now check for our LMC stars if such relation between absolute brightness and spectral subtype really exists. As Fig. 9 reveals, there is indeed some correlation, but the scatter within each subtype is large (1 mag, typically). The relation obtained by linear regression (thick shaded lines in Fig. 9) for the WNE stars is similar to the one adopted in Hamann et al. (2006), while the hydrogencontaining WNL stars in the LMC are on average less bright than $M_{v}=-7.22 \mathrm{mag}$ as used for the Galactic calibration. We note that the highest $M_{v}$ is associated with BAT99 98, which we treat as a single star, although the moderate fit quality might indicate a hidden companion (cf. Appendix B). The average $M_{v}$ value of each WN subtype is compiled in Table 3.

The bulk of "proper" WN stars populate the luminosity range from $\log \left(L / L_{\odot}\right)=5.3$ to 5.8 . The hydrogen containing stars are mainly found on the cool side of the zero-age main-sequence (ZAMS), where all WN stars are of late subtypes (WNL). The hydrogen-free stars, all of early subtypes (WNE), gather at the hot side of the ZAMS and near the theoretical zero-age mainsequence for helium stars (He-ZAMS).

In the Galactic sample, the group of WNL stars with hydrogen was not encountered in this luminosity range, possibly because of the erroneous brightness calibration applied to those stars as discussed above.

\section{Discussion}

Our large sample offers an excellent possibility to compare the almost complete WN population of the LMC with the

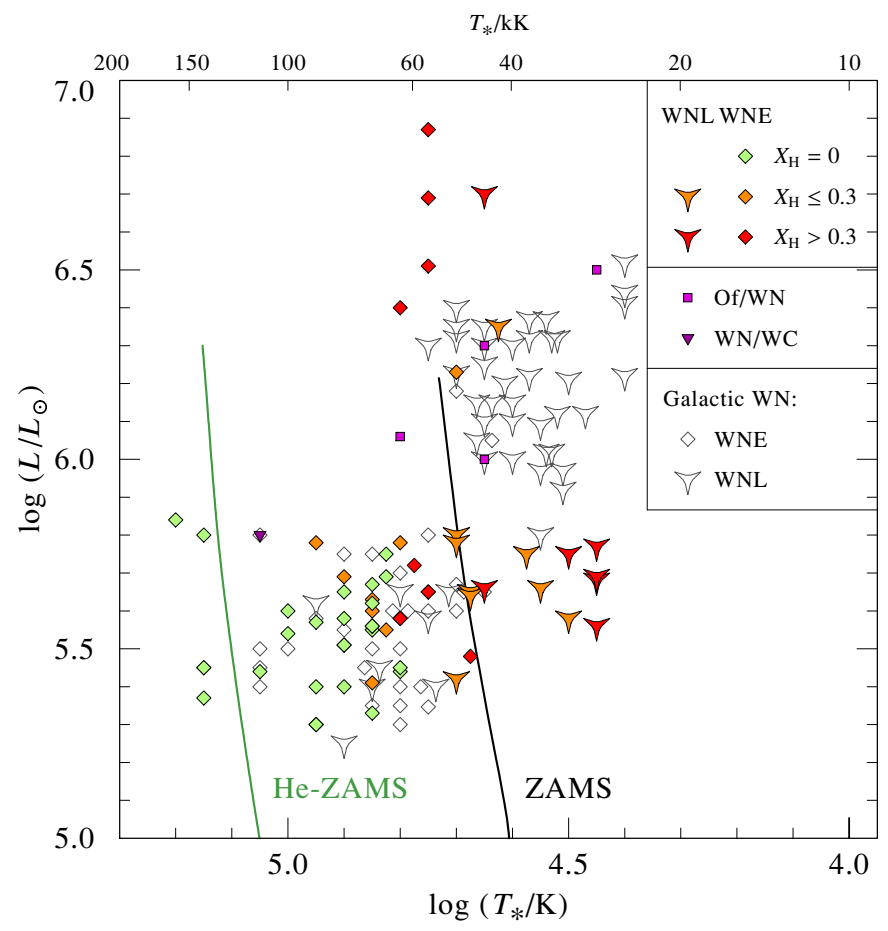

Fig. 8. HRD of the single WN stars. The color-filled symbols refer to the LMC stars analyzed in the present paper. The open symbols in the background represent the Galactic WN stars for comparison (cf. inlet).

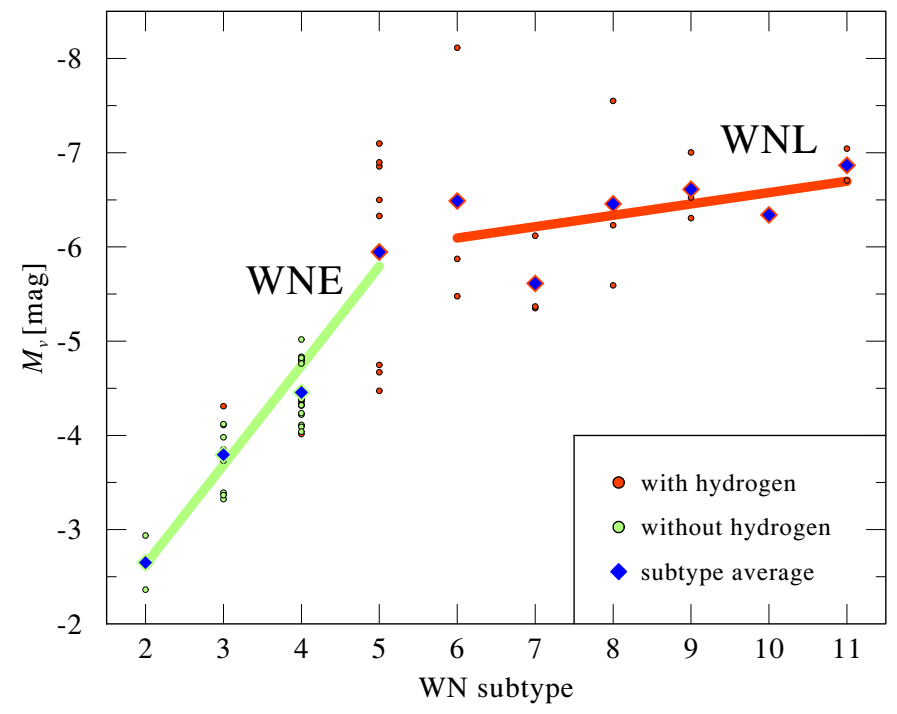

Fig. 9. Absolute visual magnitudes (narrowband color as defined by Smith 1968) versus spectral subtype number for the putatively single stars in our sample. Colors code the absence (green/light) or presence (red/dark) of hydrogen. While small symbols represent individual stars, the thick symbols mark the average $M_{v}$ of each subtype. The thick lines indicate linear fits to the early (WN2-5) and late (WN6-11) subtypes, respectively.

predictions of the stellar evolution theory. Figure 10 shows the HRD of our program stars in comparison to the stellar evolution tracks calculated by the Geneva group for LMC metallicity. In the version shown here, stellar evolution models account for the effects of rotation, but neglect the metallicity scaling in the WR phase (Meynet \& Maeder 2005).

The individual evolution phases are distinguished by different drawing styles, according to the chemical composition at the 
Table 3. Averaged absolute visual magnitudes of each WN subtype.

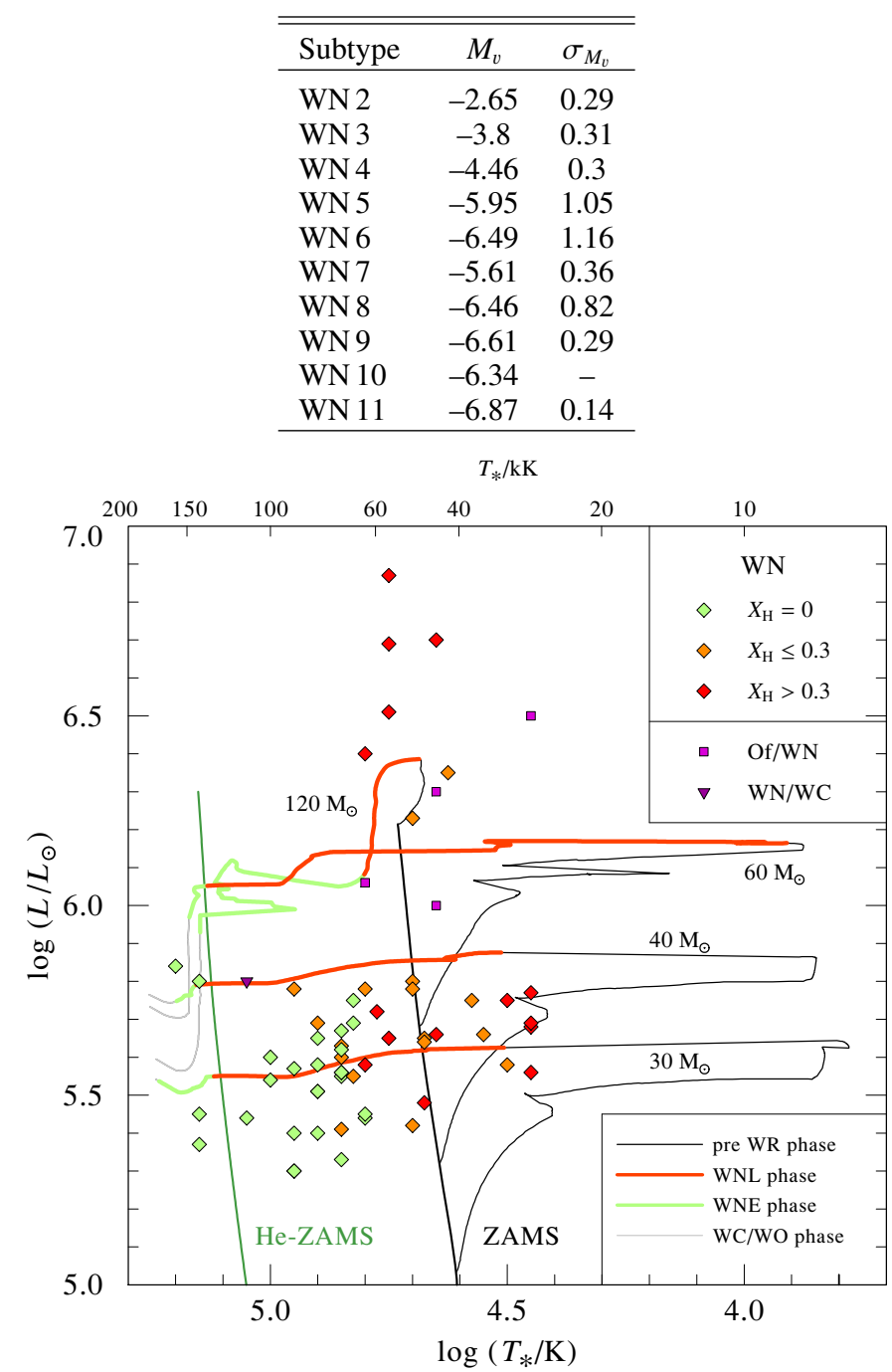

Fig. 10. HRD with the single WN stars (discrete symbols) and stellar evolution tracks from Meynet \& Maeder (2005), which account for the effects of rotation. The labels indicate the initial mass. The color coding of the tracks during the WR phases (thick lines) corresponds to the filling color of the symbols, which reflects the observed atmospheric composition (see inlet).

stellar surface. At hydrogen surface abundances $X_{\mathrm{H}}>0.4$ (mass fraction), the star is considered to be in a pre-WR phase represented by a thin black line. The WNL stage, which per definition initiates when the hydrogen abundance drops below 0.4 (mass fraction) in the atmosphere, is highlighted by thick red lines. Hydrogen abundances below 0.05 (mass fraction) are considered to correspond to the WNE stage, and the track is plotted as a thick green line. Finally, for carbon abundances above 0.2 (mass fraction), the star reaches the WC and WO phase and the track is drawn as a gray line.

Until now, we classified stars as WNE and WNL according to their spectroscopic subtype. With regard to stellar evolution, the terms "WNE" and "WNL" are defined differently and refer only to the atmospheric composition, i.e., the absence or presence of hydrogen. Note also that the temperature axis in Fig. 10 refers to the stellar temperature, i.e., to the effective temperature related to the hydrostatic core, but not to the photosphere (cf. Sect. 3).
Let us first look at the very luminous stars, separated from the others by a gap at about $\log \left(L / L_{\odot}\right)=5.9$. These stars can be explained by tracks similar to the one for $120 M_{\odot}$ shown in Fig. 10. This track stays close to the ZAMS, increases in luminosity until it enters the WNL stage, then drops, and finally evolves toward the helium main sequence after having lost all hydrogen. While the highest initial mass for which tracks are provided by Meynet \& Maeder $(2005)$ is $120 M_{\odot}$, the most luminous star of this group (BAT99 108), if indeed a single star, requires about $300 M_{\odot}$, as already pointed out by Crowther et al. (2010).

Similar to the WN stars in the Galaxy, only objects with a substantial amount of hydrogen are found at luminosities of $\log \left(L / L_{\odot}\right)>5.9$, although, according to stellar evolution models (Meynet \& Maeder 2005; Yusof et al. 2013), hydrogen-free stars are also expected in this parameter regime. This mismatch might be partly explained by very luminous type IIn supernovae (SN). Smith (2008) unveiled that the progenitors of these rare SN are probably high-mass stars $\left(M_{\text {init }}>50 M_{\odot}\right)$ that explode with a significant amount of hydrogen left in their stellar atmospheres. The observational evidence discussed by this author argues for episodic mass loss prior to the type IIn $\mathrm{SNe}$ in excess of typical WR mass-loss rates, suggesting that progenitor candidates are classical LBVs. Since the WN stars observed in this mass range seem to be rather normal WN stars, apart from their high luminosities, the origin and fate of the very massive stars still remains puzzling.

With the exception of the $120 M_{\odot}$ track, all stellar evolution tracks (for LMC metallicities) presented by Meynet \& Maeder (2005) evolve toward the red supergiant (RSG) stage, but barely reach the corresponding temperature range before evolving back to the hot part of the HRD. Nevertheless, in this paper, we denote all evolution stages in the cool part of the HRD as RSG even if the tracks only pass the blue supergiant and LBV domain of the HRD. As can be seen in Fig. 10, luminosities of about 6.2 dex are reached by the post RSG evolution of stars with initially $60 M_{\odot}$ (Meynet \& Maeder 2005). However, no "cool" WNL stars are found in this range, which might indicate that the RSG stage is in fact not reached in this mass range, possibly because of the LBV instability barrier.

The bulk of WN stars are found in the luminosity range $\log \left(L / L_{\odot}\right)=5.3 \ldots 5.8$. The upper end of this range corresponds quite well to the post-RSG track with initially $40 M_{\odot}$. Interestingly, this upper mass limit agrees well with analyses of WC stars in the LMC by Gräfener et al. (1998) and Crowther et al. (2002).

The lowest initial mass for which a post-RSG track is provided by Meynet \& Maeder (2005) for LMC metallicities is $30 M_{\odot}$. The luminosities of the analyzed WN stars show a lower cutoff at about $10^{5.3} L_{\odot}$, which rather corresponds to an initial mass of about $20 M_{\odot}$. Meynet \& Maeder (2005), based on interpolation of their tracks, estimated that the minimum initial mass for reaching WR phases is about $25 M_{\odot}$ for LMC metallicity. This limit seems to be too high, compared to our results. The older Geneva tracks, which did not account for rotation (Schaerer et al. 1993), are even more contradictory; these tracks fail to reach post-RSG stages for initial masses below $60 M_{\odot}$. We also compared our empirical HRD positions with non rotating models from Eldridge \& Vink (2006). These models predict WR stars for initial masses exceeding $33 M_{\odot}$.

As stated in Sect. 1, it is theoretically expected that the evolution of massive stars depends on their initial metallicity. Stellar evolution models predict a higher minimum for the initial mass of WR stars with decreasing metallicity due to the 
reduced mass loss by stellar winds anticipated at lower metallicities. Indeed, our study shows that the mass-loss rate of an average WN star in the LMC is lower than in the Galaxy (see Sect. 5.2 for details). However, Fig. 8 illustrates that the initial masses for the LMC WN stars are comparable to their Galactic counterparts. Thus, the conventional expectation that WN stars in the LMC originate from a higher mass range compared to their Galactic twins is not supported from our sample.

In the mass range from $20 M_{\odot}$ to $40 M_{\odot}$, the stellar evolution in the Galaxy and the LMC results in comparable HRD positions for the WN stars, although the mass-loss rates and the metallicity are lower in the LMC. This may be attributed to the relatively small differences between the metallicity of these two galaxies. Another implication might be that the WN stars and their progenitors rotate faster in the LMC than in the Galaxy, because their lower mass-loss rates imply a reduced loss of angular momentum. A faster rotation increases the WR lifetime and decreases the minimum initial mass for the WR phase, mainly due to a more efficient internal mixing (Maeder \& Meynet 2005).

Do the stellar evolution calculations successfully predict the observed number ratio between WNE and WNL stars? We used the four tracks from Meynet \& Maeder (2005) for computing synthetic populations, assuming a Salpeter initial mass function and a constant star formation rate (see HGL06, for similar simulations of the Galactic WR stars). From our simulations, we expect roughly $20 \%$ of all WN stars in the WNE phase, i.e., much less than the $40 \%$ observed. Even worse, almost half of these WNE stars originate from the tracks for 60 and $120 M_{\odot}$ initial mass and thus lie at luminosities where no WNE stars at all are found in our sample.

The synthetic population yields about the same number of WC stars as WNE stars. In fact, the WNE stars residing in the LMC are twice as frequent as the LMC-WC stars. Moreover, half of these WC stars are predicted to evolve from $120 M_{\odot}$ initial mass, which does not match the low WC luminosities actually observed (Gräfener et al. 1998; Crowther et al. 2002). Based on stellar evolution models, Eldridge \& Vink (2006) show that a better agreement with the observed $\mathrm{WC} / \mathrm{WN}$ ration can be achieved by means of a metallicity scaling of the mass-loss rate during the WR phase. However, like the Geneva models, these models also fail to reproduce the observed luminosity ranges for the different WR subtypes.

The color-coded evolution tracks in Fig. 10 reveal a discrepancy in stellar temperature between these tracks and the HRD position of the WNE stars. Theoretically, it is expected that the hydrogen-free WNE stars are located on the He-ZAMS. Instead, a clear gap is seen between most of these stars and the He-ZAMS. This discrepancy in the effective temperature is attributed to the long known "radius problem" of hydrogen-free WR stars. The observed WR radii are up to an order of magnitude larger than the radii predicted by stellar evolution models. We note that the effective temperature of the stellar evolution models refer to the hydrostatic core radius whereas the effective temperature of our atmosphere models is defined at the inner boundary radius $R_{*}$, where the Rosseland optical depth reaches 20 (cf. Sect. 3). However, this cannot be the decisive reason because $R_{*}$ is close to the hydrostatic core radius as long as $R_{*}$ is located in the hydrostatic part of the wind, which is the case for most of our final-fit models. A solution for this radius problem has been studied by Gräfener et al. (2012). These authors show that an inflated subphotospheric layer, which incorporates the effect of clumping, can bring the observed WR radii in agreement with the theory.
The binaries in our sample have been thoroughly identified, but some may have escaped detection, as discussed in Sect.2.2. Moreover, a single star could be the product of binary evolution, e.g., a merger or a binary system where the companion already exploded (Sana et al. 2012). The consequences of binary evolution with respect to the population of WR stars have been discussed by various authors (e.g., Paczyński 1967; Vanbeveren et al. 2007; Eldridge et al. 2008, 2013). According to these authors, the minimum initial mass for the WR phases is considerably decreased in binary systems due to a significant pre-WR mass-loss through Roche lobe overflow. If the least luminous stars of our sample had evolved through the binary channel, this would explain the discrepancy with the minimum initial mass of WR stars as predicted from single star evolution.

Alternatively, single star evolution may produce WR stars from lower initial masses when higher initial rotation velocities (more than the $300 \mathrm{~km} \mathrm{~s}^{-1}$ as assumed by Meynet \& Maeder 2005) are adopted. More plausibly, mass-loss rates that are enhanced over those usually adopted during the RSG stage promote the evolution of massive stars toward the blue part of the HRD (e.g., Vanbeveren et al. 1998, 2007; Georgy 2012).

Binary evolution was shown to significantly affect the WC/WN ratio (e.g., Vanbeveren et al. 2007; Eldridge et al. 2008). Eldridge et al. (2008) demonstrate that, while their single star models reproduce the observed $\mathrm{WC} / \mathrm{WN}$ ratio, the binary population models predict too many WN stars. The bias from close binary evolution on the WN population should be subject to future investigations.

The WNE and WC stars are thought to be the progenitors of type Ib and type Ic supernovae (e.g., Gaskell et al. 1986; Begelman \& Sarazin 1986), if they do not directly collapse to a black hole without a bright SN. The first tentative identification of a WR star as a type Ib SN progenitor has been reported recently for SNiPTF13bvn (Cao et al. 2013). Alternatively, Eldridge et al. (2013) show that the bulk of the SNe of type Ibc can originate from relatively low-mass pure helium stars whose hydrogen-rich atmospheres have been stripped by close binary interactions. Our empirical HDR positions (Fig. 10) suggest that the LMC-WNE stars and their successors are restricted to initial masses below $40 M_{\odot}$ similar to the situation in our Galaxy (cf. Sander et al. 2012).

Summarizing the discussion on stellar evolution, we found some general agreement between our WN analyses and the Geneva tracks for LMC metallicity that account for stellar rotation (Meynet \& Maeder 2005). However, in a quantitative sense, the stellar evolution tracks are not consistent with our empirical results. The discrepancies refer especially to the range of initial masses required for reaching the respective WR phases, and to the number ratios and luminosities of the different WR subtypes. Interestingly, similar conclusions have been drawn from our study of the Galactic WN stars and the comparison with the corresponding Geneva tracks (HGL06). Moreover, Sander et al. (2012) analyzed the Galactic WC stars and found that their relatively low luminosities are not reproduced by the Geneva tracks. Calculations by Vanbeveren et al. (1998), who adopted higher mass-loss rates during the RSG stage, yielded a better agreement. We may also mention here the work of Hunter et al. (2008), who determined the chemical composition of 135 early B-type stars in the LMC to test the prediction of rotationally induced mixing. They demonstrated that about $40 \%$ of their sample do not agree with the predicted correlation between rotation and nitrogen enrichment. Hence it seems that massive-star evolution is still not fully understood. 


\section{Summary and conclusions}

1. The spectra of 107 stars in the LMC have been analyzed by means of state-of-the-art model atmospheres.

2. The sample contains $102 \mathrm{WR}$ stars of the nitrogen sequence (WN, including five Of/WN), and thus comprises nearly the complete population of WN stars known in the LMC.

3. Of these WN stars, 63 do not show any indications of multiplicity, and thus are putatively single stars.

4. Two groups of WN stars can be distinguished from their luminosity: a small group (12\%) of very luminous stars $\log \left(L / L_{\odot}\right)>5.9$, and a large group $(88 \%)$ populating the range of moderate luminosities between $\log \left(L / L_{\odot}\right)=$ $5.3 \ldots 5.8$.

5. Of the 63 single, $27 \mathrm{WN}$ stars (i.e., $43 \%$ ) do not show hydrogen in their wind. These hydrogen-free stars are only found in the group with moderate luminosities.

6. Stellar radii are generally larger, and effective temperatures correspondingly lower, than predicted from stellar evolution models. This may indicate a subphotospheric inflation, as discussed in the recent literature.

7. The bulk of WN stars (with moderate luminosities) seem to be in a post-RSG evolution phase.

8. According to their luminosities in the range $\log \left(L / L_{\odot}\right)=$ 5.3 ... 5.8, these WN stars originate from initial stellar masses between $20 M_{\odot}$ and $40 M_{\odot}$. This mass range is similar to the range found for the Galaxy. Hence, the expected metallicity dependence of the evolution is not seen.

9. Stellar evolution tracks, when accounting for rotationally induced mixing, in principle, can explain the extremely luminous WN stars as well as the bulk of WN stars with moderate luminosities. The former evolve directly from the ZAMS, while the latter go through the RSG stage. However, the stellar evolution models still fail to correctly reproduce the observed ranges of luminosities and initial masses.

Acknowledgements. We would like to thank the referee, John Eldridge, for his constructive comments that have significantly improved the present work. This research made use of the SIMBAD database, operated at CDS, Strasbourg, France, and of data products from the Two Micron All Sky Survey, which is a joint project of the University of Massachusetts and the Infrared Processing and Analysis Center/California Institute of Technology, funded by the National Aeronautics and Space Administration and the National Science Foundation (NASA). This work is partly based on INES data from the IUE satellite, and on observations with the Spitzer Space Telescope, which is operated by the Jet Propulsion Laboratory, California Institute of Technology under a contract with NASA. This research made also use of NASA's Astrophysics Data System and of the VizieR catalog access tool, CDS, Strasbourg, France. Some of the data presented in this paper were retrieved from the Mikulski Archive for Space Telescopes (MAST). STScI is operated by the Association of Universities for Research in Astronomy, Inc., under NASA contract NAS5-26555. Support for MAST for non-HST data is provided by the NASA Office of Space Science via grant NNX09AF08G. The work also based on data made available through Wikimbad, hosted in the LAOG, France (http: //wikimbad.org). L.M.O. acknowledges the funding by DLR grant 50 OR 1302.

\section{References}

Allen, D. A., \& Glass, I. S. 1976, ApJ, 210, 666

Asplund, M., Grevesse, N., Sauval, A. J., \& Scott, P. 2009, ARA\&A, 47, 481

Barlow, M. J., \& Hummer, D. G. 1982, in Wolf-Rayet Stars: Observations, Physics, Evolution, eds. C. W. H. De Loore, \& A. J. Willis, IAU Symp., 99, 387

Barniske, A., Oskinova, L. M., \& Hamann, W.-R. 2008, A\&A, 486, 971

Begelman, M. C., \& Sarazin, C. L. 1986, ApJ, 302, L59

Bonanos, A. Z., Massa, D. L., Sewilo, M., et al. 2009, AJ, 138, 1003

Breysacher, J. 1981, A\&AS, 43, 203

Breysacher, J. 1986, A\&A, 160, 185

Breysacher, J., Azzopardi, M., \& Testor, G. 1999, A\&AS, 137, 117

Cao, Y., Kasliwal, M. M., Arcavi, I., et al. 2013 ApJ, 775, L7
Chené, A.-N., Moffat, A. F. J., Cameron, C., et al. 2011, ApJ, 735, 34

Chini, R., Hoffmeister, V. H., Nasseri, A., Stahl, O., \& Zinnecker, H. 2012, MNRAS, 424, 1925

Cowley, A. P., \& Hutchings, J. B. 1978, PASP, 90, 636

Cowley, A. P., Crampton, D., Hutchings, J. B., \& Thompson, I. B. 1984, PASP, 96, 968

Crowther, P. A. 2006, in Stellar Evolution at Low Metallicity: Mass Loss, Explosions, Cosmology, eds. H. J. G. L. M. Lamers, N. Langer, T. Nugis, \& K. Annuk, ASP Conf. Ser., 353, 157

Crowther, P. A., \& Dessart, L. 1998, MNRAS, 296, 622

Crowther, P. A., \& Hadfield, L. J. 2006, A\&A, 449, 71

Crowther, P. A., \& Smith, L. J. 1997, A\&A, 320, 500

Crowther, P. A., \& Walborn, N. R. 2011, MNRAS, 416, 1311

Crowther, P. A., Hillier, D. J., \& Smith, L. J. 1995a, A\&A, 293, 172

Crowther, P. A., Smith, L. J., \& Willis, A. J. 1995b, A\&A, 304, 269

Crowther, P. A., Dessart, L., Hillier, D. J., Abbott, J. B., \& Fullerton, A. W. 2002, A\&A, 392, 653

Crowther, P. A., Schnurr, O., Hirschi, R., et al. 2010, MNRAS, 408, 731

Cutri, R. M., Skrutskie, M. F., van Dyk, S., et al. 2012, VizieR Online Data Catalog: II/281

Dalton, G. B., Caldwell, M., Ward, A. K., et al. 2006, in SPIE Conf. Ser., 6269

de Koter, A., Heap, S. R., \& Hubeny, I. 1997, ApJ, 477, 792

Dopita, M. A., Bell, J. F., Chu, Y.-H., \& Lozinskaya, T. A. 1994, ApJS, 93, 455

Doran, E. I., \& Crowther, P. A. 2011, Bull. Soc. Roy. Sci. Liege, 80, 129

Doran, E. I., Crowther, P. A., de Koter, A., et al. 2013, A\&A, 558, A134

Duflot, M. 2010, VizieR Online Data Catalog: V/131

Dufour, R. J., Shields, G. A., \& Talbot, Jr., R. J. 1982, ApJ, 252, 461

Eldridge, J. J., \& Vink, J. S. 2006, A\&A, 452, 295

Eldridge, J. J., Izzard, R. G., \& Tout, C. A. 2008, MNRAS, 384, 1109

Eldridge, J. J., Fraser, M., Smartt, S. J., Maund, J. R., \& Crockett, R. M. 2013,

MNRAS, 436, 774

Emerson, J., McPherson, A., \& Sutherland, W. 2006, The Messenger, 126, 41 Evans, C. J., Taylor, W. D., Hénault-Brunet, V., et al. 2011, A\&A, 530, A108 Foellmi, C., Moffat, A. F. J., \& Guerrero, M. A. 2003a, MNRAS, 338, 360

Foellmi, C., Moffat, A. F. J., \& Guerrero, M. A. 2003b, MNRAS, 338, 1025

Foellmi, C., Moffat, A. F. J., \& Marchenko, S. V. 2006, A\&A, 447, 667

Gamen, R., Gosset, E., Morrell, N., et al. 2006, A\&A, 460, 777

Gaskell, C. M., Cappellaro, E., Dinerstein, H. L., et al. 1986, ApJ, 306, L77

Georgy, C. 2012, A\&A, 538, L8

Girard, T. M., van Altena, W. F., Zacharias, N., et al. 2011, AJ, 142, 15

Glass, I. S. 1984, MNRAS, 209, 759

Gosset, E., Nazé, Y., Claeskens, J.-F., et al. 2005, A\&A, 429, 685

Gräfener, G., \& Hamann, W.-R. 2005, A\&A, 432, 633

Gräfener, G., \& Hamann, W.-R. 2007, in Massive Stars in Interactive Binaries, eds. N. St.-Louis, \& A. F. J. Moffat, ASP Conf. Ser., 367, 131

Gräfener, G. \& Hamann, W.-R. 2008, A\&A, 482, 945

Gräfener, G., Hamann, W.-R., Hillier, D. J., \& Koesterke, L. 1998, A\&A, 329, 190

Gräfener, G., Koesterke, L., \& Hamann, W.-R. 2002, A\&A, 387, 244

Gräfener, G., Vink, J. S., de Koter, A., \& Langer, N. 2011, A\&A, 535, A56

Gräfener, G., Owocki, S. P., \& Vink, J. S. 2012, A\&A, 538, A40

Guerrero, M. A., \& Chu, Y.-H. 2008a, ApJS, 177, 216

Guerrero, M. A., \& Chu, Y.-H. 2008b, ApJS, 177, 238

Gvaramadze, V. V., Kniazev, A. Y., \& Fabrika, S. 2010, MNRAS, 405, 1047

Hamann, W.-R., \& Gräfener, G. 2004, A\&A, 427, 697

Hamann, W.-R., \& Koesterke, L. 1998, A\&A, 335, 1003

Hamann, W.-R., \& Koesterke, L. 2000, A\&A, 360, 647

Hamann, W.-R., Gräfener, G., \& Liermann, A. 2006, A\&A, 457, 1015

Haschke, R., Grebel, E. K., \& Duffau, S. 2011, AJ, 141, 158

Heap, S. R., Altner, B., Ebbets, D., et al. 1991, ApJ, 377, L29

Heap, S. R., Ebbets, D., Malumuth, E. M., et al. 1994, ApJ, 435, L39

Heger, A., \& Langer, N. 2000, ApJ, 544, 1016

Heydari-Malayeri, M., Courbin, F., Rauw, G., Esslinger, O., \& Magain, P. 1997, A\&A, 326, 143

Hillier, D. J. 1991, A\&A, 247, 455

Hillier, D. J., \& Miller, D. L. 1999, ApJ, 519, 354

Howarth, I. D. 1983, MNRAS, 203, 301

Humphreys, R. M., \& Davidson, K. 1994, PASP, 106, 1025

Hunter, I., Dufton, P. L., Smartt, S. J., et al. 2007, A\&A, 466, 277

Hunter, I., Brott, I., Lennon, D. J., et al. 2008, ApJ, 676, L29

Hyland, A. R., Thomas, J. A., \& Robinson, G. 1978, AJ, 83, 20

Ignace, R., Oskinova, L. M., \& Foullon, C. 2000, MNRAS, 318, 214

Ignace, R., Oskinova, L. M., \& Brown, J. C. 2003, A\&A, 408, 353

Kato, D., Nagashima, C., Nagayama, T., et al. 2007, PASJ, 59, 615

Koenigsberger, G., Moffat, A. F. J., \& Auer, L. H. 2003, Rev. Mex. Astron. Astrofis., 39, 213

Koesterke, L., Hamann, W.-R., Wessolowski, U., \& Schmutz, W. 1991, A\&A, 248, 166 
Korn, A. J., Nieva, M. F., Daflon, S., \& Cunha, K. 2005, ApJ, 633, 899

Kudritzki, R. P., Pauldrach, A., Puls, J., \& Abbott, D. C. 1989, A\&A, 219, 205

Kurt, C. M., \& Dufour, R. J. 1998, in Rev. Mex. Astron. Astrof. Conf. Ser. 7, eds. R. J. Dufour, \& S. Torres-Peimbert, 202

Larsen, S. S., Clausen, J. V., \& Storm, J. 2000, A\&A, 364, 455

Liermann, A., \& Hamann, W.-R. 2008, in Clumping in Hot-Star Winds, Proc. International Workshop, Potsdam, Germany, eds. W.-R. Hamann, A. Feldmeier, \& L. M. Oskinova, 247

Liermann, A., Hamann, W.-R., Oskinova, L. M., Todt, H., \& Butler, K. 2010, A\&A, 524, A82

Lommen, D., Yungelson, L., van den Heuvel, E., Nelemans, G., \& Portegies Zwart, S. 2005, A\&A, 443, 231

Madore, B. F., \& Freedman, W. L. 1998, ApJ, 492, 110

Maeder, A., \& Meynet, G. 2005, A\&A, 440, 1041

Martín-Hernández, N. L., Vermeij, R., \& van der Hulst, J. M. 2005, A\&A, 433, 205

Martins, F., Hillier, D. J., Paumard, T., et al. 2008, A\&A, 478, 219

Massey, P. 2002, ApJS, 141, 81

Massey, P., Waterhouse, E., \& DeGioia-Eastwood, K. 2000, AJ, 119, 2214

Massey, P., Puls, J., Pauldrach, A. W. A., et al. 2005, ApJ, 627, 477

Meynet, G., \& Maeder, A. 2003, A\&A, 404, 975

Meynet, G., \& Maeder, A. 2005, A\&A, 429, 581

Moffat, A. F. J. 1989, ApJ, 347, 373

Moffat, A. F. J., Niemela, V. S., Phillips, M. M., Chu, Y.-H., \& Seggewiss, W. 1987, ApJ, 312, 612

Nazé, Y., Rauw, G., Manfroid, J., Chu, Y.-H., \& Vreux, J.-M. 2003a, A\&A, 401, L13

Nazé, Y., Rauw, G., Manfroid, J., Chu, Y.-H., \& Vreux, J.-M. 2003b, A\&A, 408, 171

Neugent, K. F., Massey, P., \& Morrell, N. 2012, AJ, 144, 162

Niedzielski, A., \& Skorzynski, W. 2002, Acta Astron., 52, 81

Niedzielski, A., Nugis, T., \& Skorzynski, W. 2004, Acta Astron., 54, 405

Niemela, V. S. 1991, in Wolf-Rayet Stars and Interrelations with Other Massive Stars in Galaxies, eds. K. A. van der Hucht, \& B. Hidayat, IAU Symp., 143, 201

Niemela, V. S., Seggewiss, W., \& Moffat, A. F. J. 1995, in Wolf-Rayet Stars: Binaries; Colliding Winds; Evolution, eds. K. A. van der Hucht, \& P. M. Williams, IAU Symp., 163, 251

Niemela, V. S., Seggewiss, W., \& Moffat, A. F. J. 2001, A\&A, 369, 544

Nota, A., Pasquali, A., Drissen, L., et al. 1996, ApJS, 102, 383

Nugis, T., Crowther, P. A., \& Willis, A. J. 1998, A\&A, 333, 956

O'Connell, R. 2008, in HST Proposal, 11360

Oskinova, L. M., Ignace, R., Hamann, W.-R., Pollock, A. M. T., \& Brown, J. C. 2003, A\&A, 402, 755

Oskinova, L. M., Gayley, K. G., Hamann, W.-R., et al. 2012, ApJ, 747, L25

Paczyński, B. 1967, Acta Astron., 17, 355

Parker, J. W. 1993, AJ, 106, 560

Pasquali, A., Langer, N., Schmutz, W., et al. 1997, ApJ, 478, 340

Pasquali, A., Nota, A., \& Clampin, M. 1999, A\&A, 343, 536

Pauldrach, A., Puls, J., \& Kudritzki, R. P. 1986, A\&A, 164, 86

Pauldrach, A. W. A., Kudritzki, R. P., Puls, J., Butler, K., \& Hunsinger, J. 1994, A\&A, 283, 525

Piatti, A. E., \& Geisler, D. 2013, AJ, 145, 17

Pietrzyński, G., Graczyk, D., Gieren, W., et al. 2013, Nature, 495, 76

Puls, J., Urbaneja, M. A., Venero, R., et al. 2005, A\&A, 435, 669
Puls, J., Markova, N., Scuderi, S., et al. 2006, A\&A, 454, 625

Raassen, A. J. J., van der Hucht, K. A., Mewe, R., et al. 2003, A\&A, 402, 653

Reid, W. A., \& Parker, Q. A. 2012, MNRAS, 425, 355

Sana, H., de Mink, S. E., de Koter, A., et al. 2012, Science, 337, 444

Sana, H., de Koter, A., de Mink, S. E., et al. 2013a, A\&A, 550, A107

Sana, H., van Boeckel, T., Tramper, F., et al. 2013b, MNRAS, 432, L26

Sander, A., Hamann, W.-R., \& Todt, H. 2012, A\&A, 540, A144

Schaerer, D., Meynet, G., Maeder, A., \& Schaller, G. 1993, A\&AS, 98, 523

Schmutz, W., Hamann, W., \& Wessolowski, U. 1989, A\&A, 210, 236

Schmutz, W., Leitherer, C., Hubeny, I., et al. 1991, ApJ, 372, 664

Schnurr, O., Moffat, A. F. J., St-Louis, N., Morrell, N. I., \& Guerrero, M. A. 2008, MNRAS, 389, 806

Schnurr, O., Chené, A.-N., Casoli, J., Moffat, A. F. J., \& St-Louis, N. 2009a, MNRAS, 397, 2049

Schnurr, O., Moffat, A. F. J., Villar-Sbaffi, A., St-Louis, N., \& Morrell, N. I. 2009b, MNRAS, 395, 823

Seaton, M. J. 1979, MNRAS, 187, 73

Seggewiss, W., Moffat, A. F. J., \& Lamontagne, R. 1991, A\&AS, 89, 105

Selman, F., Melnick, J., Bosch, G., \& Terlevich, R. 1999, A\&A, 341, 98

Shenar, T., Hamann, W.-R., \& Todt, H. 2014, A\&A, 562, A118

Skinner, S. L., Zhekov, S. A., Güdel, M., Schmutz, W., \& Sokal, K. R. 2012, AJ, 143,116

Skrutskie, M. F., Cutri, R. M., Stiening, R., et al. 2006, AJ, 131, 1163

Smith, L. F. 1968, MNRAS, 140, 409

Smith, L. F., Shara, M. M., \& Moffat, A. F. J. 1996, MNRAS, 281, 163

Smith, L. J., Norris, R. P. F., \& Crowther, P. A. 2002, MNRAS, 337, 1309

Smith, N. 2008, in IAU Symp. 250, eds. F. Bresolin, P. A. Crowther, \& J. Puls, 193

Smith, R. C., Points, S., Chu, Y.-H., et al. 2005, in AAS Meeting Abstracts, \#145.01, BAAS, 37

Stahl, O., Wolf, B., Leitherer, C., et al. 1984, A\&A, 140, 459

Stevens, I. R., Blondin, J. M., \& Pollock, A. M. T. 1992, ApJ, 386, 265

Subramaniam, A. 2005, A\&A, 430, 421

Testor, G., Llebaria, A., \& Debray, B. 1988, The Messenger, 54, 43

Taylor, W. D., Evans, C. J., Sana, H., et al. 2011, A\&A, 530, L10

Todt, H., Kniazev, A. Y., Gvaramadze, V. V., et al. 2013, MNRAS, 430, 2302

Torres-Dodgen, A. V., \& Massey, P. 1988, AJ, 96, 1076

Townsley, L. K., Broos, P. S., Feigelson, E. D., Garmire, G. P., \& Getman, K. V. 2006, AJ, 131, 2164

Trundle, C., Dufton, P. L., Hunter, I., et al. 2007, A\&A, 471, 625

Vanbeveren, D., De Donder, E., van Bever, J., van Rensbergen, W., \& De Loore, C. 1998, New Astron., 3, 443

Vanbeveren, D., Van Bever, J., \& Belkus, H. 2007, ApJ, 662, L107

Vink, J. S. 2007, A\&A, 469, 707

Vink, J. S., \& de Koter, A. 2005, A\&A, 442, 587

Vink, J. S., Heger, A., Krumholz, M. R., et al. 2013 [arXiv: 1302.2021 ]

Walborn, N. R. 1982, ApJ, 256, 452

Walborn, N. R., MacKenty, J. W., Saha, A., White, R. L., \& Parker, J. W. 1995, ApJ, 439, L47

Walborn, N. R., Drissen, L., Parker, J. W., et al. 1999, AJ, 118, 1684

Weis, K. 2003, A\&A, 408, 205

Willis, A. J., Crowther, P. A., Fullerton, A. W., et al. 2004, ApJS, 154, 651

Yoon, S.-C., Dierks, A., \& Langer, N. 2012, A\&A, 542, A113

Yusof, N., Hirschi, R., Meynet, G., et al. 2013, MNRAS, 433, 1114

Zaritsky, D., Harris, J., Thompson, I. B., \& Grebel, E. K. 2004, AJ, 128, 1606 
R. Hainich et al.: The Wolf-Rayet stars in the Large Magellanic Cloud

\section{Appendix A: Additional tables}

Table A.1. Available spectra for WN stars in the LMC.

\begin{tabular}{|c|c|c|c|c|c|c|c|}
\hline No. & $\Delta \lambda / \AA$ & Instrument & Flux & Archive & HST Proposal ID & PI & No. of stars \\
\hline 1 & 1090-2330 & HST-FOS & abs. & MAST & 4260 & C. Leitherer & 5 \\
\hline 2 & $1090-2330$ & HST-FOS & abs. & MAST & 5702 & C. Leitherer & 1 \\
\hline 3 & $\begin{array}{l}1120-1720 \\
6300-6860\end{array}$ & HST-STIS & abs. & MAST & 9412 & P. Massey & 2 \\
\hline 4 & $1140-1730$ & HST-STIS & abs. & MAST & 9434 & J. Lauroesch & 1 \\
\hline 5 & $1150-3200$ & IUE & abs. & INES & - & diverse & 63 \\
\hline 6 & $1160-1750$ & HST-GHRS & abs. & MAST & 5297 & S. Heap & 2 \\
\hline 7 & $1160-1750$ & HST-FOS & abs. & MAST & 6018 & S. Heap & 1 \\
\hline 8 & $1200-1760$ & HST-GHRS & abs. & MAST & 5157 & D. Ebbets & 2 \\
\hline 9 & $\begin{array}{l}2020-2030 \\
2050-2070\end{array}$ & HST-GHRS & abs. & MAST & 5887 & K. Roth & 2 \\
\hline 10 & $3020-3300$ & HST-STIS & abs. & MAST & 9412 & P. Massey & 1 \\
\hline 11 & $3240-4780$ & HST-FOS & abs. & MAST & 6508 & N. Walborn & 1 \\
\hline 12 & $3240-4780$ & HST-FOS & abs. & MAST & 6417 & P. Massey & 6 \\
\hline 13 & $3240-4780$ & HST-FOS & abs. & MAST & 6032 & N. Walborn & 1 \\
\hline 14 & $3240-6820$ & HST-FOS & abs. & MAST & 6018 & S. Heap & 5 \\
\hline 15 & $3240-6820$ & HST-FOS & abs. & MAST & 6110 & C. Leitherer & 1 \\
\hline 16 & $3400-7300$ & CTIO & abs. & VizieR & - & A. V. Torres-Dodgen & 53 \\
\hline 17 & $3670-6000$ & AAT-RGO & norm. & AAT & - & Smith, P. Crowther & 28 \\
\hline 18 & $3700-6800$ & diverse & norm. & private & - & C. Foellmi & 62 \\
\hline 19 & $3770-9055$ & ESO-EFOSC & norm. & private & - & U. Wessolowski & 19 \\
\hline 20 & $4000-5500$ & diverse & norm. & private & - & O. Schnurr & 42 \\
\hline 21 & $\begin{array}{l}4310-4590 \\
6300-6870\end{array}$ & HST-STIS & abs. & MAST & 7739 & P. Massey & 1 \\
\hline
\end{tabular}


Table A.2. LMC WN stars analyzed in this work.

\begin{tabular}{|c|c|c|c|c|c|c|c|}
\hline BAT99 & Brey & Figure & Observations $^{a}$ & BAT99 & Brey & Figure & Observations $^{a}$ \\
\hline 001 & 01 & C. 1 & $5,16,18,19$ & 071 & 60 & C.28 & $5,16,18$ \\
\hline 002 & 02 & C. 1 & 5,18 & 072 & 61 & C. 28 & 18 \\
\hline 003 & 03 & C. 2 & $5,16,18$ & 073 & 63 & C. 29 & 18 \\
\hline 005 & 04 & C. 2 & $5,16,18,19$ & 074 & $63 \mathrm{a}$ & C. 29 & 18 \\
\hline 006 & 05 & C. 3 & 5 & 075 & 59 & C. 30 & 5,18 \\
\hline 007 & 06 & C. 3 & $5,16,17,18,19$ & 076 & 64 & C. 30 & $2,5,16,17,19,20$ \\
\hline 012 & $10 \mathrm{a}$ & C. 4 & $5,10,17,20$ & 077 & 65 & C. 31 & $5,16,20$ \\
\hline 013 & - & C. 4 & $1,5,17,20$ & 078 & $65 \mathrm{~b}$ & C. 31 & 11,18 \\
\hline 014 & 11 & C. 5 & 5,18 & 079 & 57 & C. 32 & $5,16,17,20$ \\
\hline 015 & 12 & C. 5 & $5,16,17,18,19$ & 080 & $65 c$ & C. 32 & 20 \\
\hline 016 & 13 & C.6 & $5,16,17,19$ & 081 & $65 \mathrm{a}$ & C. 33 & 5,18 \\
\hline 017 & 14 & C. 6 & $5,16,18$ & 082 & 66 & C. 33 & $5,16,18$ \\
\hline 018 & 15 & C.7 & $5,16,18$ & 086 & 69 & C. 34 & 16,18 \\
\hline 019 & 16 & C.7 & $5,16,17,18,19$ & 088 & $70 \mathrm{a}$ & C. 34 & 18 \\
\hline 021 & 17 & C. 8 & 18 & 089 & 71 & C. 35 & $5,17,20$ \\
\hline 022 & 18 & C. 8 & $5,16,17,20$ & 091 & 73 & C. 35 & $13,18,20$ \\
\hline 023 & - & C.9 & 18,17 & 092 & 72 & C.36 & $5,16,20$ \\
\hline 024 & 19 & C.9 & $5,16,18$ & 093 & $74 \mathrm{a}$ & C. 36 & 5,20 \\
\hline 025 & $19 \mathrm{a}$ & C. 10 & 18 & 094 & 85 & C. 37 & $16,18,19$ \\
\hline 026 & 20 & C. 10 & $5,16,18$ & 095 & 80 & C. 37 & $5,16,17,20$ \\
\hline 027 & 21 & C. 11 & $5,16,18$ & 096 & 81 & C. 38 & 20 \\
\hline 029 & 23 & C. 11 & $5,16,18$ & 097 & - & C. 38 & 20 \\
\hline 030 & 24 & C. 12 & $5,16,17,19,20$ & 098 & 79 & C. 39 & 20 \\
\hline 031 & 25 & C. 12 & $5,16,18$ & 099 & 78 & C. 39 & $3,12,20$ \\
\hline 032 & 26 & C. 13 & $5,16,17,19,20$ & 100 & 75 & C. 40 & 12,20 \\
\hline 033 & - & C. 13 & $1,5,9,17,20$ & 102 & 87 & C. 40 & 20 \\
\hline 035 & 27 & C. 14 & $5,16,18,19$ & 103 & 87 & C. 41 & 20 \\
\hline 036 & 29 & C. 14 & $5,16,17,18,19$ & 104 & 76 & C. 41 & $12,20,21$ \\
\hline 037 & 30 & C. 15 & 16,18 & 105 & 77 & C. 42 & $5,12,20$ \\
\hline 040 & 33 & C. 15 & $16,17,18$ & 106 & 82 & C. 42 & 8,14 \\
\hline 041 & 35 & C.16 & $5,16,18$ & 107 & 86 & C.43 & 5,20 \\
\hline 042 & 34 & C. 16 & $5,16,18$ & 108 & 82 & C. 43 & 8,14 \\
\hline 043 & 37 & C. 17 & $5,16,18,19$ & 109 & 82 & C. 44 & 6,14 \\
\hline 044 & 36 & C. 17 & $5,16,17,20$ & 110 & 82 & C. 44 & 6,14 \\
\hline 046 & 38 & C. 18 & $5,16,18$ & 111 & 82 & C. 45 & 7,14 \\
\hline 047 & 39 & C. 18 & 18 & 112 & 82 & C. 45 & 12 \\
\hline 048 & 40 & C. 19 & $5,16,18,19$ & 113 & - & C. 46 & 12,20 \\
\hline 049 & $40 \mathrm{a}$ & C. 19 & 5,18 & 114 & - & C. 46 & 12,20 \\
\hline 050 & 41 & C. 20 & 18 & 116 & 84 & C. 47 & 20 \\
\hline 051 & 42 & C. 20 & $5,16,18$ & 117 & 88 & C. 47 & $5,16,18,19$ \\
\hline 054 & $44 a$ & C. 21 & 17,20 & 118 & 89 & C. 48 & $4,5,16,20$ \\
\hline 055 & - & C. 21 & $1,5,9,15,17,20$ & 119 & 90 & C. 48 & $5,16,17,20$ \\
\hline 056 & 46 & C. 22 & $5,16,18$ & 120 & 91 & C. 49 & $1,5,17,20$ \\
\hline 057 & 45 & C. 22 & $5,16,18$ & 122 & 92 & C. 49 & $5,16,17,18$ \\
\hline 058 & 47 & C. 23 & $5,16,17,19,20$ & 124 & $93 \mathrm{a}$ & C. 50 & 18 \\
\hline 059 & 48 & C. 23 & $5,16,18,19$ & 126 & 95 & C. 50 & 18 \\
\hline 060 & 49 & C. 24 & 18,16 & 128 & 96 & C. 51 & 16,18 \\
\hline 062 & 51 & C. 24 & 18,16 & 129 & 97 & C. 51 & 18 \\
\hline 063 & 52 & C. 25 & $5,16,17,18,19$ & 130 & - & C. 52 & $5,17,20$ \\
\hline 064 & 53 & C. 25 & 18 & 131 & 98 & C. 52 & 5,18 \\
\hline 065 & 55 & C. 26 & 17,18 & 132 & 99 & C.53 & $5,16,18$ \\
\hline 066 & 54 & C. 26 & 16,18 & 133 & - & C. 53 & $1,5,17,20$ \\
\hline 067 & 56 & C. 27 & $16,18,19$ & 134 & 160 & C. 54 & $5,16,18$ \\
\hline 068 & 58 & C. 27 & $3,5,16,20$ & & & & \\
\hline
\end{tabular}

Notes. ${ }^{(a)}$ Sources of observations; the numbers refer to the entries in Table A.1. 
R. Hainich et al.: The Wolf-Rayet stars in the Large Magellanic Cloud

Table A.3. Number of ionizing photons and Zanstra temperatures for WN stars in the LMC.

\begin{tabular}{|c|c|c|c|c|c|c|c|c|c|c|c|}
\hline \multirow[t]{2}{*}{ BAT99 } & \multicolumn{2}{|c|}{$\mathrm{HI}$} & \multirow{2}{*}{$\begin{array}{c}\mathrm{He} \mathrm{I} \\
\log Q \\
{\left[\mathrm{~s}^{-1}\right]}\end{array}$} & \multicolumn{2}{|c|}{ He II } & \multirow[t]{2}{*}{ BAT99 } & \multicolumn{2}{|c|}{$\mathrm{HI}_{\mathrm{I}}$} & \multirow{2}{*}{$\begin{array}{c}\mathrm{He} \mathrm{I} \\
\log Q \\
{\left[\mathrm{~s}^{-1}\right]}\end{array}$} & \multicolumn{2}{|c|}{ He II } \\
\hline & $\begin{array}{c}\log Q \\
{\left[\mathrm{~s}^{-1}\right]}\end{array}$ & $\begin{array}{c}T_{\text {Zanstra }} \\
{[\mathrm{K}]}\end{array}$ & & $\begin{array}{c}\log Q \\
{\left[\mathrm{~s}^{-1}\right]}\end{array}$ & $\begin{array}{c}T_{\text {Zanstra }} \\
{[\mathrm{K}]}\end{array}$ & & $\begin{array}{c}\log Q \\
{\left[\mathrm{~s}^{-1}\right]}\end{array}$ & $\begin{array}{c}T_{\text {Zanstra }} \\
{[\mathrm{K}]}\end{array}$ & & $\begin{array}{l}\log Q \\
{\left[\mathrm{~s}^{-1}\right]}\end{array}$ & $\begin{array}{c}T_{\text {Zanstra }} \\
{[\mathrm{K}]}\end{array}$ \\
\hline 001 & 49.16 & 67981 & 48.92 & 38.01 & 20714 & 071 & 49.86 & 66841 & 49.47 & - & - \\
\hline 002 & 49.22 & 84379 & 49.04 & 47.52 & 80957 & 072 & 49.68 & 75882 & 49.37 & 46.16 & 51455 \\
\hline 003 & 49.37 & 59612 & 49.08 & - & - & 073 & 49.59 & 62324 & 49.17 & - & - \\
\hline 005 & 49.30 & 84379 & 49.12 & 47.60 & 80957 & 074 & 49.57 & 84145 & 49.31 & 46.49 & 57497 \\
\hline 006 & 50.31 & 60738 & 49.82 & 39.80 & 21522 & 075 & 49.44 & 62180 & 49.11 & - & - \\
\hline 007 & 49.67 & 57958 & 49.45 & - & - & 076 & 48.97 & 29996 & - & - & - \\
\hline 012 & 49.62 & 52361 & 49.00 & 39.26 & 21416 & 077 & 50.55 & 45662 & 49.79 & 39.77 & 20389 \\
\hline 013 & - & - & - & 29.98 & - & 078 & 49.58 & 57920 & 49.25 & - & - \\
\hline 014 & 49.75 & 69099 & 49.40 & 39.08 & 21545 & 079 & 49.92 & 41794 & 48.91 & - & - \\
\hline 015 & 49.43 & 61599 & 49.16 & 38.59 & 21001 & 080 & 50.17 & 46145 & 49.38 & 38.36 & 18931 \\
\hline 016 & 49.61 & 43162 & 48.84 & - & - & 081 & 49.30 & 48116 & 48.58 & 37.72 & 19339 \\
\hline 017 & 49.57 & 62210 & 49.22 & - & - & 082 & 49.40 & 77611 & 49.17 & 39.27 & 22783 \\
\hline 018 & 49.51 & 64794 & 49.20 & - & - & 086 & 49.21 & 69922 & 48.90 & - & - \\
\hline 019 & 50.01 & 68147 & 49.74 & - & - & 088 & 49.66 & 70897 & 49.43 & 38.82 & 21288 \\
\hline 021 & 50.19 & 71312 & 49.84 & - & - & 089 & 49.60 & 44234 & 48.90 & - & - \\
\hline 022 & 48.50 & 22617 & 40.85 & - & - & 091 & 49.25 & 45949 & 48.58 & - & - \\
\hline 023 & 49.43 & 69922 & 49.12 & - & - & 092 & 50.74 & 46069 & 49.95 & 38.59 & 18501 \\
\hline 024 & 49.39 & 55730 & 49.10 & 38.17 & 20193 & 093 & 49.65 & 45639 & 48.89 & 38.73 & 20177 \\
\hline 025 & 49.43 & 69718 & 49.09 & - & - & 094 & 49.64 & 57867 & 49.40 & 38.75 & 20782 \\
\hline 026 & 49.49 & 57392 & 49.15 & - & - & 095 & 49.78 & 41802 & 48.85 & - & - \\
\hline 027 & 51.18 & 76456 & 50.87 & 47.26 & 47867 & 096 & 50.09 & 41175 & 48.96 & - & - \\
\hline 029 & 49.38 & 62180 & 49.05 & - & - & 097 & 50.06 & 45563 & 49.29 & 38.98 & 19936 \\
\hline 030 & 49.47 & 45563 & 48.69 & 37.58 & 18810 & 098 & 50.47 & 45042 & 49.67 & 38.96 & 19286 \\
\hline 031 & 49.20 & 61206 & 48.89 & - & - & 099 & 49.67 & 46213 & 48.89 & 38.12 & 19294 \\
\hline 032 & 49.76 & 45314 & 48.98 & 37.30 & 18063 & 100 & 49.97 & 44385 & 49.17 & 37.72 & 18289 \\
\hline 033 & 49.16 & 21529 & 41.08 & - & - & 102 & 50.58 & 44687 & 49.76 & 38.54 & 18554 \\
\hline 035 & 49.48 & 60383 & 49.16 & - & - & 103 & 50.07 & 47527 & 49.34 & - & - \\
\hline 036 & 49.58 & 65035 & 49.30 & - & - & 104 & 49.93 & 67173 & 49.55 & 40.23 & 23274 \\
\hline 037 & 49.52 & 70919 & 49.25 & - & - & 105 & 50.22 & 52407 & 49.60 & - & - \\
\hline 040 & 49.50 & 63102 & 49.12 & - & - & 106 & 50.37 & 57074 & 49.89 & - & - \\
\hline 041 & 49.46 & 63646 & 49.20 & 38.79 & 21348 & 107 & 49.78 & 33161 & 47.89 & - & - \\
\hline 042 & 51.88 & 77528 & 51.57 & 48.76 & 55866 & 108 & 50.73 & 58502 & 50.25 & 40.78 & 22436 \\
\hline 043 & 49.74 & 68087 & 49.39 & - & - & 109 & 50.55 & 58502 & 50.07 & 40.60 & 22436 \\
\hline 044 & 49.44 & 43479 & 48.56 & 37.36 & 18455 & 110 & 50.04 & 51984 & 49.41 & 40.10 & 22156 \\
\hline 046 & 49.32 & 61500 & 48.93 & - & - & 111 & 50.01 & 45707 & 49.25 & 39.34 & 20578 \\
\hline 047 & 49.46 & 68540 & 49.21 & 39.06 & 22005 & 112 & 50.35 & 57278 & 49.85 & 39.74 & 21220 \\
\hline 048 & 49.25 & 55443 & 48.96 & 38.67 & 21190 & 113 & 49.92 & 53011 & 49.30 & - & - \\
\hline 049 & 50.21 & 76811 & 49.90 & 47.21 & 57157 & 114 & 50.31 & 68041 & 49.92 & 46.59 & 48366 \\
\hline 050 & 49.51 & 58615 & 49.03 & - & - & 116 & 50.93 & 64922 & 50.54 & 41.50 & 23758 \\
\hline 051 & 49.17 & 67981 & 48.91 & 38.69 & 21847 & 117 & 50.28 & 66229 & 49.89 & 40.56 & 23206 \\
\hline 054 & 49.35 & 36243 & 47.70 & - & - & 118 & 50.48 & 45254 & 49.71 & 37.82 & 17821 \\
\hline 055 & 48.39 & 21258 & 41.51 & - & - & 119 & 50.39 & 46621 & 49.65 & - & - \\
\hline 056 & 49.43 & 59756 & 49.10 & - & - & 120 & 48.68 & 26530 & - & - & - \\
\hline 057 & 49.26 & 59612 & 48.97 & - & - & 122 & 50.07 & 48441 & 49.42 & - & - \\
\hline 058 & 49.46 & 46183 & 48.72 & 37.37 & 18569 & 124 & 49.33 & 64016 & 48.95 & - & - \\
\hline 059 & 50.34 & 76184 & 50.02 & - & - & 126 & 50.33 & 72906 & 50.01 & - & - \\
\hline 060 & 49.66 & 66531 & 49.27 & 39.38 & 22141 & 128 & 49.30 & 64076 & 49.05 & - & - \\
\hline 062 & 49.29 & 60383 & 48.97 & - & - & 129 & 50.08 & 84145 & 49.82 & 47.00 & 57497 \\
\hline 063 & 49.46 & 62875 & 49.08 & - & - & 130 & 48.22 & 20638 & 39.86 & - & - \\
\hline 064 & 49.94 & 72906 & 49.62 & - & - & 131 & 49.54 & 59756 & 49.21 & - & - \\
\hline 065 & 49.63 & 62210 & 49.28 & - & - & 132 & 49.43 & 54053 & 49.12 & - & - \\
\hline 066 & 49.65 & 85134 & 49.43 & 46.51 & 56916 & 133 & 48.29 & 21099 & - & - & - \\
\hline 067 & 49.78 & 47709 & 49.06 & - & - & 134 & 49.37 & 59612 & 49.08 & - & - \\
\hline 068 & 49.76 & 45699 & 48.99 & 38.66 & 19906 & & & & & & \\
\hline
\end{tabular}

Notes. In these cases, where only an insignificant number of ionizing photons can escape the stellar atmosphere, a hyphen is used for $T_{\text {Zanstra }}$ and $\log Q$. 


\section{Appendix B: Comments on individual stars}

Preliminary remark on mass-loss rates. In our models, clumping is parameterized by means of the "density contrast" $D$ (see Sect. 3). We adopt $D=10$ throughout our analyses. Other authors used different assumptions for the degree of clumping, or neglected the wind inhomogeneities. As a consequence of the scaling invariance described by Eq. (2), the impact of the clumping contrast on the empirical mass-loss rates that are derived from recombination lines is simply $\dot{M} \propto D^{-1 / 2}$. Therefore, when comparing mass-loss rates with the results from other authors in the following text, we scale their $\dot{M}$ values to our assumptions of $D=10$.

BAT99 1 is classified as WN3b in the BAT99 catalog. It shows no periodic radial velocity variations, but seems to be a runaway star, according to Foellmi et al. (2003b). We find no hints for binarity in the spectra and treat them as single stars. Bonanos et al. (2009) derived a stellar temperature of $T_{*}=85 \mathrm{kK}$ on the basis of CMFGEN models from Smith et al. (2002), which is slightly lower than $T_{*}=89 \mathrm{kK}$, as derived in our analysis. The same value has been obtained by HK2000 with a previous version of our code. Willis et al. (2004) have used their FUSE spectra to derive a terminal velocity of $v_{\infty}=2745 \mathrm{~km} \mathrm{~s}^{-1}$ from the black edge of the P Cygni profiles present in the far UV (FUV), whereas Niedzielski et al. (2004) estimated terminal velocities of $v_{\infty}=1265-2506 \mathrm{~km} \mathrm{~s}^{-1}$ from the P Cygni lines in the IUE-range with the same method. We prefer $v_{\infty}=1600 \mathrm{~km} \mathrm{~s}^{-1}$ from the width of the optical emission lines in accordance with HK2000. A terminal velocity of $v_{\infty}=2754 \mathrm{~km} \mathrm{~s}^{-1}$ would result in emission lines which are considerably broader than observed (see Sect. 4.3 for details).

BAT992 is one of the two WN2 stars (the other one is BAT99 49) in the LMC, and clearly among the hottest stars of the sample. Actually, BAT99 2 is one of only two WR stars in the LMC able to ionize a He II-region (Nazé et al. 2003b). These authors conclude from the nebular He II-flux that the exciting star delivers $4 \times 10^{47} \mathrm{He}$ II ionizing photons per second. This agrees with our final model, which produces $3.3 \times 10^{47} \mathrm{He}$ II ionizing photons per second.

A model with a stellar temperature of 90-100 kK (Nazé et al. 2003a) cannot reproduce the observed spectra. The synthetic spectra below $T_{*}=110 \mathrm{kK}$ show C IV $\lambda 1548$ and C IV $\lambda 5801$ lines, which are not observed. All appropriate models with stellar temperatures above $T_{*}=110 \mathrm{kK}$ fall into the regime of parameter degeneracy of the model grid (see Sect. 4.2). In this case, the determination of the temperature was based on a slightly better fit for the N v $\lambda 1242$ and N v $\lambda 4603$ lines at $T_{*}=141 \mathrm{kK}$. Between fits with temperatures of $130 \mathrm{kK}$ and $160 \mathrm{kK}$, the slope of the optical/UV continuum (and thus the inferred reddening parameter) hardly changes, but the luminosity increases from $\log \left(L / L_{\odot}\right)=5.35$ to 5.58 due to the bolometric correction.

Foellmi et al. (2003b) reported the detection of hydrogen emission in the spectrum of BAT992. We cannot exclude a hydrogen mass-fraction of $X_{\mathrm{H}} \sim 0.1$, but the observation is perfectly consistent with zero hydrogen. The presence of hydrogen would be unexpected for a WN star of such high effective temperature and, thus, advanced evolution stage.

Foellmi et al. (2003b) find that the radial velocity of BAT99 2 differs from the mean $v_{\text {rad }}$ of their WN sample by about $-120 \mathrm{~km} \mathrm{~s}^{-1}$ and suggest that this star might be a runaway object.
BAT993 is listed as spectral type WN4b in the BAT99 catalog. For the first time, stellar and wind parameters for this object are presented here.

BAT995 is the second of the two WN2b stars (Foellmi et al. 2003b) in the LMC. BAT99 2 and BAT99 5 exhibit very similar spectra that can be reproduced by the same grid model. The temperature of BAT995 was previously determined to be only $71 \mathrm{kK}$ by HK2000, but with today's line-blanketed models much higher temperatures are adequate, as discussed above for BAT992. However, we note that this star is located within the regime of parameter degeneracy (see Sect.4.2). Massey et al. (2000) suggest an initial mass of $M_{\text {init }}>40 M_{\odot}$, while the Geneva tracks in Fig. 10 indicate slightly less than $30 M_{\odot}$.

BAT99 5 is suspected to have an OB-companion by Smith et al. (1996), in reference to absorption features visible in the spectrum from Torres-Dodgen \& Massey (1988), but the authors mention that these features could also be artifacts from the subtraction of nebular lines. According to Foellmi et al. (2003b), the spectrum shows no radial velocity variations. Like BAT992, BAT99 5 was not detected in X-rays by Guerrero \& Chu (2008b). As we also find no indications of a companion in the spectrum, we consider this star to be single. If the spectroscopic twin of BAT99 5, namely BAT992, is indeed a runaway star as suggested by Foellmi et al. (2003b), the single star evolution of BAT99 5 and the binary evolution of BAT99 2 have led to almost identical products.

BAT99 6 is one of the WN binary systems (period of $2 \mathrm{~d}$ ) listed in BAT99, but has been demoted since the publication of this catalog. Niemela et al. (2001) reclassified it as O3 $\mathrm{f}^{*}+\mathrm{O}$ on the basis of its optical spectrum, suggesting that the system contains four stars (two close pairs). On the other hand, Koenigsberger et al. (2003) conclude that the system does not comprise more than two luminous stars. Unfortunately, we do not have optical spectra of this star, so that the stellar parameters are derived from the UV spectrum and the photometry alone. We achieved a reasonable fit, although this binary is fitted as a single star. According to Niemela et al. (2001) the total mass of the system is probably over $80 M_{\odot}$.

BAT99 7, classified as WN4b (BAT99), shows strong emission lines with a round line shape. These line shapes can only be reproduced assuming a high rotational velocity of $v_{\text {rot }}=$ $2200 \mathrm{~km} \mathrm{~s}^{-1}$. Thus, this star is a prototype for the so-called "round line" stars, which are characterized by strong and broad emission lines with round line profiles. The correlation of these line shapes with the stellar rotation has recently been investigated by Shenar et al. (2014). These authors confirm that rotation can account for the spectral characteristics of BAT997, but only in connection with a strong magnetic fields that force the wind to co-rotate.

We derive a stellar temperature of $T_{*}=158 \mathrm{kK}$, which is the hottest of all WN4 stars. However, we note that this stars falls into the regime of parameter degeneracy (see Sect. 4.2). The derived stellar temperature is conspicuously higher than values obtained by Koesterke et al. $\left(1991, T_{*}=90 \mathrm{kK}\right)$, using pure helium models. HK2000 obtained a temperature of $T_{*}=100 \mathrm{kK}$ with unblanketed model atmospheres.

BAT99 12 is a transition type O2 If*/WN5 star, according to Crowther \& Walborn (2011). Schnurr et al. (2008) argued that the star is most likely a runaway, as already suggested by Massey et al. (2005). Several spectra are at hand for the analysis of this 
star. In the UV range an HST spectrum and multiple observations with the IUE satellite are available, although only two IUE shortwavelength spectra are in accordance with the HST observation. All IUE long-wavelength spectra exhibit a substantial offset to the rest of the observed SED. Therefore, we have ignored these IUE data. In the optical spectral range, the AAT spectrum and one spectrum observed by Foellmi et al. (2003b) complement each other in wavelength coverage. All spectra can be fitted with the same model, which gives us confidence in the derived stellar parameters.

We derived a stellar temperature of $T_{*}=50 \mathrm{kK}$, confirming the value obtained by Doran \& Crowther (2011) and Doran et al. (2013). A lower temperature limit of $T_{*}>42 \mathrm{kK}$ was derived by Massey et al. (2005) with the FASTWIND model atmosphere code (Puls et al. 2005). However, at these cooler temperatures neither the N IV $\lambda 4060$ nor the $\mathrm{N} v \lambda \lambda 4604,4620$ lines can be satisfactorily reproduced. In comparison to the study carried out by Doran et al. (2013), we derived the same luminosity, while the mass-loss rate is 0.1 dex lower. In contrast, Massey et al. (2005) derived a mass-loss rate that is 0.23 dex higher. We achieve the best fit with synthetic spectra for a hydrogen mass-fraction of $X_{\mathrm{H}}=0.5$, which is 0.1 dex lower than previously derived by Doran et al. (2013).

Schnurr et al. (2008) reported radial velocity variations of this star with a period of $3.2 \mathrm{~d}$. For the companion, no spectral features are detected. The SED is well reproduced by a singlestar model, thus we expect that the companion does not contribute much to the bolometric luminosity of the binary system.

BAT99 13 is the only WN10 star (BAT99) in the LMC. It has been analyzed before by Crowther et al. (1995b) and Pasquali et al. (1997). The former derived a stellar temperature of $T_{*}=$ $29.7 \mathrm{kK}$ with unblanketed atmosphere models. The analysis by Pasquali et al. (1997) with line blanketed models obtained a higher temperature of $T_{*}=33 \mathrm{kK}$. Our best fit is obtained with a model of $T_{*}=28 \mathrm{kK}$. At a temperature of $T_{*}=32 \mathrm{kK}$ (one grid step higher) the fit is also reasonable, with the exception of a considerably over-predicted He II $\lambda 4686$ line. Thus, we preferred the model with the lower temperature $\left(T_{*}=28 \mathrm{kK}\right)$. Since the emission line strength of the He II $\lambda 4686$ line is slightly too low at these temperatures, the "real" temperature is probably marginally higher. But this has only a minor impact on the other stellar parameters. The mass-loss rate derived in our analysis is more than a factor of three below the previous values obtained by Crowther et al. (1995b) and Pasquali et al. (1997).

Bonanos et al. (2009) compared the observed SED with the continua of a $45 \mathrm{kk}$ WN model calculated by Smith et al. (2002) with CMFGEN. From this comparison they found an infrared excess, which cannot be confirmed by our analysis (see Fig. C.4). These different results arise from the high temperature assumed by Bonanos et al. (2009) for this WN10 star. Models calculated with this temperature cannot consistently reproduce the observed spectra.

BAT99 14 is listed as $\mathrm{WN}+\mathrm{OB}$ ? binary candidate without a period in the BAT99 catalog. Foellmi et al. (2003b) reclassified it as $\mathrm{WN} 4 \mathrm{o}(+\mathrm{OB})$, but they did not find significant periodical variations in their radial velocity data, concluding that this object is probably not a short-period binary. However, they find absorption lines superimposed on the emission lines, which they attribute to a nearby visual companion. Since our analysis is based on the same spectra, we treat this star as a binary suspect until more appropriate data are available. Stellar and wind parameters are derived in this work for the first time.
BAT99 15 is classified as WN4b (BAT99). It has already been analyzed by Koesterke et al. (1991) and HK2000. We obtained approximately the same stellar temperature as HK2000, but a factor of two lower mass-loss rate. A higher mass-loss rate, however, results in emission lines which are substantially stronger than observed. Furthermore, we derived a slightly higher color excess of $E_{b-v}=0.08 \mathrm{mag}$, which gives rise to the higher luminosity derived in our analysis.

BAT99 16 was classified as WN7h by Schnurr et al. (2008). We achieve the best fit at a stellar temperature of $T_{*}=50 \mathrm{kK}$, whereas $T_{*}=33 \mathrm{kK}, T_{*}=34.8 \mathrm{kK}$ and $T_{*}=35.5 \mathrm{kK}$ have been derived by Koesterke et al. (1991), Crowther \& Smith (1997), and HK2000, respectively. Such a low temperature, however, would completely spoil the fit of the $\mathrm{He}$ and $\mathrm{N}$ lines. For example, the line ratio of the He I $\lambda 5877$ to He II $\lambda 5412$ is much higher than observed in the model with a temperature of $35 \mathrm{kK}$. We attribute these differences to the unblanketed model atmospheres used by the former authors.

We found a mass-loss rate of $\log \left(\dot{M} /\left(M_{\odot} / \mathrm{yr}\right)\right)=-4.64$. The same value was derived by Crowther \& Smith (1997), while Koesterke et al. (1991) obtained a mass-loss rate almost a factor of two lower. HK2000, on the other hand, derived a mass-loss rate nearly a factor of two higher. We estimate a hydrogen massfraction of $X_{\mathrm{H}}=0.3$ from the best fitting models, slightly higher compared to the value derived by HK2000.

BAT99 17 is listed as WN4o in the BAT99 catalog. Bonanos et al. (2009) have analyzed this star, using CMFGEN models by Crowther (2006). They derived an effective temperature of $T_{\text {eff }}=52 \mathrm{kK}$, a luminosity of $\log \left(L / L_{\odot}\right)=5.4$ and a massloss rate of $\log \left(\dot{M} /\left(M_{\odot} / \mathrm{yr}\right)\right)=-4.85$. In our analysis, the best overall fit is achieved with a model corresponding to an effective temperature of $T_{\text {eff }}=65 \mathrm{kK}$. We note that a slightly better fit of the He II $\lambda 5412$ and the He I $\lambda 5877$ lines can be achieved at an effective temperature of $T_{\text {eff }}=55 \mathrm{kK}$, although the fit quality of all nitrogen lines and the He II $\lambda 1641,4201,4339,4542$ is reduced compared to the model with $T_{\text {eff }}=65 \mathrm{kK}$. The luminosity derived in this work is factor of two higher than the value obtained by Bonanos et al. (2009), while the mass-loss rate and the terminal velocity are nearly the same. The luminosity increase in comparison to the former study by Bonanos et al. (2009) originates from the higher temperature and thus higher bolometric correction derived in our analysis.

BAT99 18 is a WN3(h) star (Foellmi et al. 2003b), which had never been analyzed before by means of model atmospheres. We confirm the presence of hydrogen $\left(X_{\mathrm{H}}=0.2\right)$, i.e., the corresponding classification.

BAT99 19 was classified as WN4b+OB? in the BAT99 catalog. Foellmi et al. (2003b) reported a period of $17.99 \mathrm{~d}$ and specified the companion to be an O5: star. In the spectrum from Foellmi et al. (2003b), small absorption lines are superimposed on the emission lines. Due to the weakness of these lines we expect that the contribution of the companion to the bolometric luminosity is only minor. Actually, we achieved a reasonable fit with our single-star model although a high rotational velocity $\left(v_{\text {rot }} \sin i=\right.$ $2000 \mathrm{~km} \mathrm{~s}^{-1}$ ) is necessary to reproduce the round emission lines, as has already been remarked by Breysacher (1981). Thus, this star belongs to the category of the so-called "round line" stars. The correlation of these line shapes with the stellar rotation has recently been investigated by Shenar et al. (2014). 
HK2000 have derived a stellar temperature of $T_{*}=70.8 \mathrm{kK}$ one grid step lower compared to our new results, which engender a slightly better fit of the UV and optical line spectra at $T_{*}=79 \mathrm{kK}$. We attribute these differences in the models to the line blanketing not incorporated in the models by HK2000. Both results are considerably above the $50 \mathrm{kK}$ obtained by Koesterke et al. (1991) with pure helium models. The mass-loss rate presented in this paper is marginally lower compared to HK2000, if we take the higher terminal velocity $v_{\infty}=2500 \mathrm{~km} \mathrm{~s}^{-1}$ derived by these authors into account. The SED fit results in nearly the same luminosity and color excess as obtained by HK2000.

BAT9921 is listed as WN4+OB binary candidate without a period in the BAT99 catalog. Foellmi et al. (2003b) reclassified this object as $\mathrm{WN} 4 \mathrm{o}(+\mathrm{OB})$. According to these authors, the radial velocity variations are only marginal, concluding that this object is probably not a short-period binary. They further mentioned that a visual companion situated $2^{\prime \prime}$ away contributes to the observed flux and causes the absorption lines in the spectrum. We analyze the object as a single WN star, although a substantial flux contribution from the companion is expected due to the sizable absorption lines of the companion. More appropriate data is needed to ensure accurate model estimates and to verify the binary status. Until this data is available, we treat this star as a binary suspect.

BAT9922 is an LBV candidate according to Humphreys \& Davidson (1994), Crowther et al. (1995a), and Pasquali et al. (1997), which is listed as WN9h star by BAT99. So far, similar stellar parameters have been reported by Schmutz et al. (1991), Crowther et al. (1995a), and Pasquali et al. (1997). These authors derived stellar temperatures in the range from $T_{*}=28.5 \mathrm{kK} \ldots 35.2 \mathrm{kK}$ with unblanketed and blanketed model atmosphere codes, respectively. We obtain a stellar temperature of $T_{*}=32 \mathrm{kK}$, matching the hitherto known temperature range. However, we can exclude the higher as well as the lower temperature, since the He II $\lambda 4686$ line would be considerably overpredicted at $35 \mathrm{kK}$ and underpredicted at $28 \mathrm{kK}$, respectively.

This star is one of three LMC WN stars that has been detected at $24 \mu \mathrm{m}$ with the IRAC instrument aboard the Spitzer space telescope (Bonanos et al. 2009). It is known to show a huge infrared excess (Glass 1984; Stahl et al. 1984), which is also visible in our SED fit. According to Allen \& Glass (1976), Cowley \& Hutchings (1978), and Stahl et al. (1984), a M2 supergiant contributes to the near-infrared flux, and thus probably causes the infrared excess. Schmutz et al. (1991) concluded that the $\mathrm{M}$ supergiant is not physically bound to BAT9922, rather incidentally located along the same line of sight. Observations by Heydari-Malayeri et al. (1997) with ESO NTT SUSI show that the M supergiant is closer than $0.12^{\prime \prime}$, which points to a binary system according to these authors. However, no significant variations have been found in the recent radial-velocity study by Schnurr et al. (2008), which militates against a short-period binary.

A comparison of the spectrum obtained by Schnurr et al. (2008) with that shown in Cowley \& Hutchings (1978) indicates that at least one faint $\mathrm{TiO}$ band at $5167 \AA$ is visible in the spectrum from Schnurr et al. (2008). Therefore, our results for BAT99 22 are also slightly effected by the late-type supergiant, since our analysis is partially based on this spectrum. Due to the weakness of this feature, however, we consider the uncertainty of the WN parameters introduced by the contribution of the $\mathrm{M} \mathrm{su}-$ pergiant to the flux in the optical spectral range as small.
Vink (2007) found intrinsic line depolarization for the He II $\lambda 6560$ line, suggesting an asymmetry in the wind, which is probably either caused by an binary companion or rapid rotation. Weis (2003) found evidence for a nebula associate to BAT9922, which is not spatially resolved by the available observations. A circumstellar shell has already been proposed by Stahl et al. (1984). These authors concluded that the excess in the $L$ band is too large to be explained by a late supergiant only.

The UV and optical spectra (flux-calibrated) are consistent with a luminosity of $\log \left(L / L_{\odot}\right)=5.75$. Within the uncertainties, this is equal to the findings of Schmutz et al. (1991) and Crowther et al. (1995a), whereas $\log \left(L / L_{\odot}\right)=5.9$ was obtained by Pasquali et al. (1997). The best fitting model requires a massloss rate of $\log \left(\dot{M} /\left(M_{\odot} / \mathrm{yr}\right)\right)=-4.85$, which is lower but comparable with the previous results (Schmutz et al. 1991; Crowther et al. 1995a; Pasquali et al. 1997).

BAT99 23 was classified as WN3(h) by Foellmi et al. (2003b) due to the hydrogen emission detected by these authors. Our best fit is achieved with a hydrogen-free grid-model, suggesting that the hydrogen mass fraction in the atmosphere of this star is below $X_{\mathrm{H}}=0.1$. As far as we know, no analysis based on stellar atmosphere models has been published for this object yet.

BAT99 24 is listed as WN4b in the BAT99 catalog. For the first time, we present stellar and wind parameters for this star derived with modern stellar atmospheres. With a stellar temperature of $T_{*}=100 \mathrm{kK}$ and a terminal velocity of $v_{\infty}=2400 \mathrm{~km} \mathrm{~s}^{-1}$, it belongs to the hottest stars in our sample with one of the fastest winds. However, we note that this star is located within the regime of parameter degeneracy (see Sect. 4.2 for details).

BAT99 25 was classified as WN4ha by Foellmi et al. (2003b), rejecting the previous WN3 classification. Since we could not find parameters of this object in the literature for comparison, this object had probably never been analyzed before.

BAT99 26 is a WN4b star (BAT99) with stellar and wind parameters typical for early WN stars. The only exception is the mass-loss rate, which is lower compared to the average of the other WN4b stars in our sample. The parameters derived in our analysis are the first made public for this star.

BAT99 27 has a known B-type companion, although Foellmi et al. (2003b) concluded that this star is probably not a shortperiodic binary because no radial velocity variations were detected. Since absorption lines of the companion are also visible in the spectra obtained by these authors, they have slightly reclassified the system to WN5b(+B1 Ia). High resolution observations with the Wide Field Camera 3 (WFC3) aboard the HST by P. Massey (HST Proposal 12940) could not resolve this object in multiple components. Whether the B-type star is physically bound to the WR star or only located in the line of sight is still unclear.

The early B-type companion significantly contributes to the flux of the whole system. Therefore, our analysis of BAT99 27 as a single WN star is considerably affected by the companion. This is the reason that no conclusive fit could be established in this work. The disentanglement of the spectra will be a subject of a forthcoming paper. However, the emission lines have a broad and round line shape, suggesting that the WN component belongs to the so-called "round line" stars. To account for the round line shape, the synthetic spectra is convolved with a rotation profile, 
corresponding to a rotational velocity of $v_{\text {rot }}=1000 \mathrm{~km} \mathrm{~s}^{-1}$. The correlation of the line shapes with the stellar rotation has recently been investigated by Shenar et al. (2014).

BAT99 29 was revealed as a binary with a period of $2.2 \mathrm{~d}$ by Foellmi et al. (2003b) on the basis of their radial velocity studies. These authors classified this object as $\mathrm{WN} 4 \mathrm{~b}+\mathrm{OB}$. They note a reduced line intensity of the hydrogen lines due to the presence of a companion. However, a reasonable fit of the Balmer lines can also be established with a single-star model (although the $\mathrm{H} \alpha$ line is slightly overpredicted by the model), suggesting that the flux contribution of the OB companion is rather small in this binary system.

BAT9930 is listed as WN6h in the BAT99 catalog. Stellar temperatures in the range $T_{*}=33 \ldots 39.8 \mathrm{kK}$ have been derived by Koesterke et al. (1991), Crowther \& Smith (1997), and HK2000 with unblanketed stellar atmosphere models. We achieved the best fit with a stellar temperature of $T_{*}=47 \mathrm{kK}$. At this temperature, our models reproduce the observed $\mathrm{He}$ I/He II ratio as well as the $\mathrm{N}$ III/N IV ratio (with a trend to slightly higher temperatures), whereas a clear mismatch is obtained with the low temperatures derived in the previous studies. This discrepancy probably originates from the line-blanketing effect included in our models. Similar values for the luminosity and the mass-loss rate have been obtained by Koesterke et al. (1991), Crowther \& Smith (1997), and HK2000. We confirm the previous estimates of the mass-loss rate, but obtain a higher luminosity of $\log \left(L / L_{\odot}\right)=5.65$, which can be partly attributed to the hotter stellar temperature derived from our line fit. In agreement with the classification, we find a hydrogen mass-fraction of $X_{\mathrm{H}}=0.3$, which is 0.1 dex lower than previously derived by HK2000.

BAT99 31 was classified as WN4b binary candidate by Foellmi et al. (2003b). However, these authors could not derive a period from their radial velocity measurements. As we find no indications of a companion in its spectrum, we consider the contribution to the bolometric luminosity of this possible companion as negligible and analyze this object as a single WN star.

BAT99 32 is long known as a binary system with a short period of $P=1.91 \mathrm{~d}$ (Moffat 1989; Schnurr et al. 2008). It is listed with a WN6(h) spectral type in the BAT99 catalog. We note that emission lines like He II $\lambda 4686$ and He II $\lambda 5412$ exhibit an asymmetric line shape in the spectrum obtained by TorresDodgen \& Massey (1988), whereas none of these peculiarities can be detected in the spectrum by Schnurr et al. (2008), which might be an effect of the lower spectral resolution. Since we cannot with certainty estimate the flux contribution of the companion, we consider the derived physical parameters of this object as uncertain.

Fairly similar stellar parameters were obtained by Koesterke et al. (1991), Crowther \& Smith (1997), and HK2000, although the derived stellar temperatures exhibit some scatter. Our analysis, on the other hand results in a stellar temperature of $T_{*}=$ $47 \mathrm{kK}$, i.e., $7 \mathrm{kK}$ higher than the highest temperature obtained in previous studies. Similar to BAT9930, the high temperature derived in our analysis probably originates from the lineblanketing effect included in our code. Since the earlier analyses by Koesterke et al. (1991) and Crowther \& Smith (1997) are based on models that do not account for wind inhomogeneities, the mass-loss rate derived by these authors is certainly overestimated. However, the scaling of these values according to the clumping factor $D=10$ assumed in this work results in nearly the same mass-loss rate. The same is true for the results obtained by HK2000, who assumed a lower clumping factor of $D=4$. Compared to Koesterke et al. (1991), Crowther \& Smith (1997), and HK2000, we have derived a higher luminosity mainly due to the different stellar temperature obtained in our analysis.

BAT99 33 belongs to the category of Ofpe/WN9 stars (also designated as "cool slash-stars"), that were incorporated in the WNsubclass system (Crowther et al. 1995a; Crowther \& Smith 1997) as WN9-11 stars. According to Crowther \& Smith (1997), this star is exceptional, since its spectral peculiarities prevent a closer classification. A detailed discussion of the spectral morphology can be found in Crowther \& Smith (1997) and Pasquali et al. (1997). Humphreys \& Davidson (1994) list this star as a LBV candidate. No clear evidence for a circumstellar nebular was found by Weis (2003). They attributed the nebulosity previously detected by Nota et al. (1996) to a background H II region due to the low densities derived by these authors. In contrast Gvaramadze et al. (2010) found a bow-shock structure to the east of BAT9933, using archival $24 \mu \mathrm{m}$ data obtained with the Multiband Imaging Photometer (MIPS) aboard the Spitzer Space Telescope. Significant intrinsic line polarization was discovered by Vink (2007), suggesting the presence of an asymmetric stellar wind, which could explain the peculiarities visible in the spectrum of this star.

Pasquali et al. (1997) inferred a stellar temperature of $T_{*}=$ $35 \mathrm{kK}$, which is $7 \mathrm{kK}$ higher than the temperature obtained in our analysis. Our $35 \mathrm{kK}$ model completely overestimates the strength of the He II $\lambda 4686,5412$ lines in comparison to the He I lines, which unambiguously points to lower stellar temperatures. Moreover, the former authors speculated that the He I and He II lines originate from different wind components because the helium line widths do not follow their expectations. Thus, they noted that their stellar parameters may not accurately represent the physical conditions of this star. With the exception of the asymmetric He II $\lambda 4686$ line, the line width of all helium lines can be consistently reproduced in our analysis.

BAT99 35 was classified as WN3(h) by Foellmi et al. (2003b). Our analysis results in similar stellar parameters compared to the previous analysis by HK2000. The only exception is the inferred stellar temperature, which is reduced by one grid step in our present study, leading to a slightly better reproduction of the $\mathrm{He} \mathrm{I} / \mathrm{He}$ II ratio. The best fit is obtained with a hydrogen massfraction of $X_{\mathrm{H}}=0.1$, although a hydrogen-free atmosphere cannot be excluded with certainty.

BAT99 36 is one of two WNE/WCE transition type stars in the LMC that exhibit a clear carbon enhancement compared to the rest of the LMC WN sample. As noted by Foellmi et al. (2003b), the top of the Balmer lines are attenuated, which, according to these authors, could be due to a faint absorptionline companion, as already proposed by Crowther et al. (1995b). However, Foellmi et al. (2003b) note that their radial velocities measurements are consistent with a single star. We regard this object as a binary suspect until more appropriate data are available. Considering the small effect on the emission lines of the WN star, we expect that the potential companion does not contribute much to the bolometric luminosity.

We obtained quite the same stellar parameters as HK2000. Analyses prior to HK2000 suggested lower values for the stellar temperature and luminosity but nearly the same mass-loss rate (Koesterke et al. 1991; Crowther et al. 1995b). With a carbon 
abundance of $X_{\mathrm{C}}=0.003$ derived in our analysis, we can confirm the previous results obtained by Crowther et al. (1995b).

BAT99 37 has the spectral type WN3o (Foellmi et al. 2003b). Unfortunately, we do not have an interpretable UV spectrum of this star. The optical spectrum from Torres-Dodgen \& Massey (1988) is very noisy, so that reddening and luminosity cannot be determined precisely. Fitting this spectrum, the color excess is $E_{b-v}=0.7 \mathrm{mag}$, while a color excess of $E_{b-v}=0.5 \mathrm{mag}$ is necessary to obtain a good fit of the photometry measured by Crowther \& Hadfield (2006); the luminosity does not differ between the fits.

BAT99 40 is listed as WN50+O binary candidate in the BAT99 catalog. However, Foellmi et al. (2003b) did not find a radial velocity period and reclassified this star to WN4(h)a. Furthermore, they attributed the absorption lines visible in their spectra to be intrinsic to the wind of the WN star. These authors, on the other hand, have detected X-ray emission in the archival ROSAT data, whereas Guerrero \& Chu (2008b) list this object as undetected by ROSAT observations. Despite these contradicting results, we treat this star as a binary suspect until its binary status is clarified. The stellar parameters given in this work are the first derived for this object.

BAT99 41 is a WN4b star (BAT99) that has never been analyzed before. We note that this stars falls into the regime of parameter degeneracy (cf. Sect. 4.2).

BAT9942 is the brightest source in our sample, with an extremely high stellar luminosity of $\log \left(L / L_{\odot}\right)=8.0$. This is 1.06 dex higher than the stellar luminosity derived by Crowther et al. (2010) for BAT99 108 in the core of R136, and 1.2 dex higher than the value obtained by Sana et al. (2013b) for the binary BAT99 118 (R144).

BAT99 42 is a visual binary classified as WN5b(h) (Foellmi et al. 2003b) with a long known B-type supergiant (B3I) companion (Smith et al. 1996, and references therein). For the whole system, the detection of X-ray emission was reported by Guerrero \& Chu (2008b). Seggewiss et al. (1991) found a spectroscopic period of $P=30.18 \mathrm{~d}$, though this value is highly uncertain, according to the authors. Foellmi et al. (2003b), on the other hand, concluded from their radial velocity studies that the WN component is probably not a spectroscopic binary, although some scatter is present in their radial velocity data.

This object is associated with the LH 58 cluster, which is located about $1.1^{\circ}$ to the northwest of 30 Doradus. New UV observations of this cluster were obtained by P. Massey with the WFC3 aboard the HST. These high resolution images dissolve BAT99 42 in three major components (see Fig. B.1) that lie within the slit width used by Foellmi et al. (2003b) and TorresDodgen \& Massey (1988) (see Fig. B.1).

Figure B.1 even shows a small cluster around BAT99 42. This whole cluster is completely covered by the large aperture of the IUE satellite, which was used for the UV observations analyzed in this work. Thus, all available spectra represent at least the three major objects in this small cluster. A disentanglement of the spectra is beyond the scope of this paper and will be the subject of our forthcoming work. The photometry used to construct the observed SED is affected by the same problem since the core of this cluster cannot be resolved by most of the available instruments.

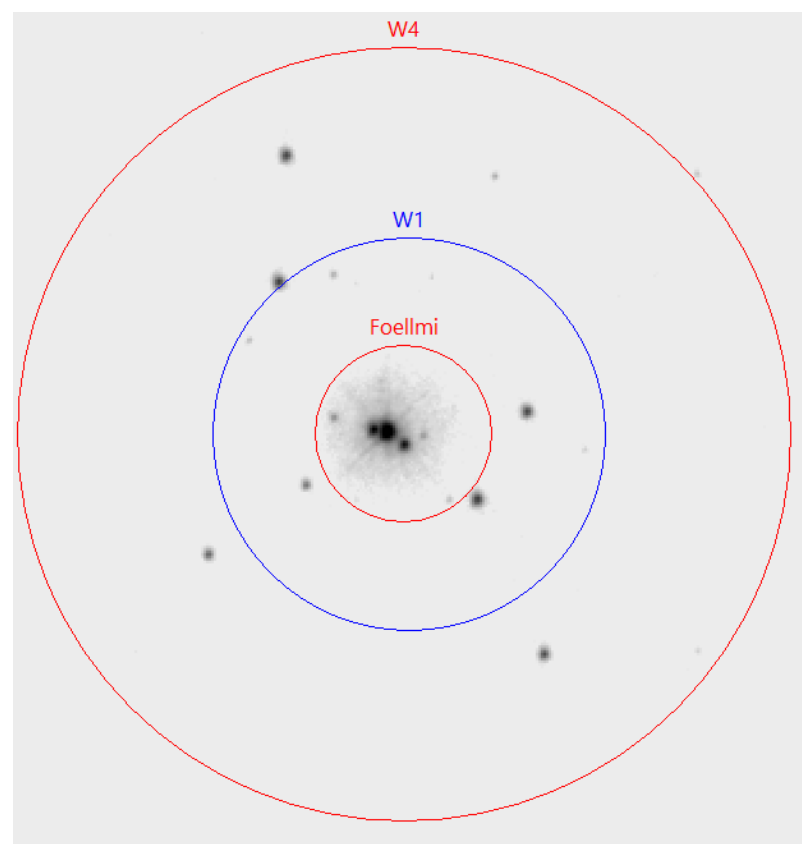

Fig. B.1. An HST-WFC3 F225W image of the region within about $10^{\prime \prime}$ distance of BAT9942. The image was requested from the HST archive. The cycles refer to the apertures of the WISE photometer (6.1" for the W1 and 12" for the W4 band, respectively) and the maximum slit width used by Foellmi et al. (2003b).

Apart from the lower signal-to-noise ratio $(\mathrm{S} / \mathrm{N})$, the fluxcalibrated spectrum obtained by Torres-Dodgen \& Massey (1988) is almost identical to the spectrum observed by Foellmi et al. (2003b). The weak round-shaped emission lines are obviously diluted by the contribution of the non-WN components to the overall flux. The round shape of the emission lines requires a convolution of the model spectrum with a rotation profile corresponding to a rotational velocity of $v_{\text {rot }} \sin i=2300 \mathrm{~km} \mathrm{~s}^{-1}$. Thus, the WN component might belong to the so-called "round line" stars.

The narrow absorption lines from the non-WR components of this system are clearly visible in the high $\mathrm{S} / \mathrm{N}$ spectrum from Foellmi et al. (2003b). These lines are relatively weak, which can be attributed to dilution effects as well. Therefore, we estimate the contribution of the WN component to the bolometric luminosity to be considerable although the non-WN components probably contribute most to the flux in the optical spectral range. Thus, this cluster probably hosts one of the most luminous WN stars in the LMC. However, without additional data it is hard to constrain the real luminosity of the WR component.

BAT99 43 was listed as WN4+OB binary candidate in the BAT99 catalog. Foellmi et al. (2003b) found a $2.8 \mathrm{~d}$ period for this double-line spectroscopic binary (SB2; Moffat 1989). We expect that the companion does not contribute much to the bolometric luminosity of this binary system (see also Foellmi et al. 2003 b), since we do not see any unambiguous features of the companion in our spectra. This object has been previously analyzed by Koesterke et al. (1991) and HK2000. These authors have derived a stellar temperature of $T_{*}=79.4 \mathrm{kK}$, one grid step above our new results. A higher stellar temperature in our analysis, however, would result in a complete mismatch of the observed N IV / N V ratio. To the contrary, the line strength of the $\mathrm{He} \mathrm{I} \lambda 5877$ line points to an even lower stellar temperature. 
Compared to HK2000, the luminosity obtained here is almost a factor of two lower, while the mass-loss rate is approximately the same.

BAT99 44 was classified as WN8ha by Schnurr et al. (2008). Bonanos et al. (2009) discovered an infrared excess by comparing the observed SED with line-blanketed atmosphere models (Smith et al. 2002), which exhibit a stellar temperature of $T_{*}=45 \mathrm{kK}$. This temperature is considerably higher than obtained by Crowther et al. (1995b), using unblanketed model atmospheres. Our analysis agrees with the estimates by Bonanos et al. (2009). However, we cannot find an infrared excess for this object. Compared to Crowther et al. (1995b), the luminosity obtained in this work is a factor of two higher, mainly due to the higher stellar temperature, while the mass-loss rate is fairly the same.

BAT99 46 is a WN4o star (BAT99), which was not spectroscopically analyzed by means of model atmospheres before.

BAT99 47 is classified as WN3b (Foellmi et al. 2003b) and had never been analyzed before. Although Foellmi et al. (2003b) could not find periodic radial velocity variations, we treat this object as a binary suspect because of the X-ray emission reported by Guerrero \& Chu (2008b). Unfortunately, we do not have fluxcalibrated spectra for BAT9947. However we have photometric data from the UV to the mid-infrared, so that the luminosity and the interstellar reddening can be well determined. We achieved a plausible fit of the SED with our single-star model. Therefore, we expect that the possible companion does not contribute much to the bolometric luminosity.

BAT99 48 is listed as a WN4b in the BAT99 catalog. This star was previously analyzed by Koesterke et al. (1991) and HK2000. The former authors have obtained a stellar temperature of $T_{*}=$ $57 \mathrm{kK}$ based on unblanketed model atmospheres, whereas the latter authors derived a stellar temperature of $T_{*}=79.4 \mathrm{kK}$ with blanketed model atmospheres. In comparison to the last work, our new analysis results in a $10 \mathrm{kK}$ higher stellar temperature, a factor of two higher mass-loss rate but nearly the same luminosity. We note that this star is located within the regime of parameter degeneracy (cf. Sect. 4.2).

BAT99 49 was identified as a SB2 binary with a period of $34 \mathrm{~d}$ (Niemela 1991). A slightly smaller period of $31.7 \mathrm{~d}$ was found by Foellmi et al. (2003b), who classified the primary as WN4:b and the companion as $08 \mathrm{~V}$. The stellar parameters that we derived for this object are to be taken with care, since we analyze this object as a single star but the companion may substantially contribute to the overall flux.

BAT99 49 is one of only two WR stars in the LMC that is able to ionize a He II region (Nazé et al. 2003b): the other one is BAT992. Nazé et al. (2003b) conclude from the nebular He II-flux that the exciting star delivers $\sim 1 \times 10^{47} \mathrm{He}$ II ionizing photons per second. This agrees with our final model, which produces $1.6 \times 10^{47} \mathrm{He}$ II ionizing photons per second. However, we note that our final model probably overestimates the number of ionizing photons, since the proper luminosity of the WN component is certainly lower than the value given in Table 2, which is derived neglecting its binary nature.
BAT9950 was classified as WN5h by Crowther \& Hadfield (2006). For this object, we have derived the stellar parameters by means of stellar atmosphere models for the first time. Unfortunately, we do not have UV spectra so the stellar parameters are derived from this normalized optical spectrum and photometry alone. Thus, the obtained luminosity is subject to higher uncertainties compared to those luminosities simultaneously derived from flux-calibrated spectra and photometry.

BAT9951 is listed with a WN3b classification in the BAT99 catalog. The spectrum of BAT99 51 is dominated by broad and round emission lines. Therefore, this object belongs to the socalled round-lined stars (cf. comment on BAT99 7). The line shapes can only be reproduced assuming a high rotational velocity of $v_{\text {rot }}=1000 \mathrm{~km} \mathrm{~s}^{-1}$ as discussed in Sect 4.3 .

Unfortunately, we do not have an interpretable UV spectrum for this stars. Its only IUE spectrum (SWP 04872) is not usable. It is noisy and lacks WR features, and does not show enough flux in relation to the photometric data and flux-calibrated spectrum obtained by Torres-Dodgen \& Massey (1988).

BAT9954 was classified as WN8ha by Schnurr et al. (2008). Unfortunately, the optical spectra at hand do not cover the $\mathrm{H} \alpha$ line. Consequently, the hydrogen abundance is determined from the higher members of the Balmer series alone. We note that the line strength of the $\mathrm{H} \beta$ line may point to a slightly higher hydrogen mass-fraction than the $X_{\mathrm{H}}=0.2$ given in Table 2 . With our line-blanketed models we derived a $7 \mathrm{kK}$ higher stellar temperature than previously obtained by Crowther \& Smith (1997) with unblanketed model atmospheres. This higher value results in a roughly $50 \%$ higher luminosity, whereas the mass-loss rate is a factor of three higher in our analysis.

BAT9955 is one of only three WN11 stars in the whole sample. According to Humphreys \& Davidson (1994), this WN star is a LBV candidate. Schnurr et al. (2008) concluded from the radial velocity of this object that it is most likely a runaway star. BAT99 55 is one of three LMC WN stars detected at $24 \mu \mathrm{m}$ with the IRAC instrument aboard the Spitzer space telescope, suggesting the presence of circumstellar dust (Bonanos et al. 2009). Crowther \& Smith (1997) and Pasquali et al. (1997) derived rather similar stellar parameters for this star. The stellar temperature and the luminosity derived in this work are in good agreement with the properties presented by Crowther \& Smith (1997). On the other hand, our study results in a $70 \%$ higher mass-loss rate, which can be attributed to the higher terminal wind velocity derived in our analysis.

BAT9956 is a WN4b star (BAT99). This object had never been spectroscopically analyzed by means of model atmospheres before.

BAT9957 is another WN4b star (BAT99) with typical stellar parameters, which was not analyzed by means of model atmospheres before.

BAT9958 is a WN7h star (Schnurr et al. 2008) studied by Koesterke et al. (1991), Crowther \& Smith (1997), HK2000 and Bonanos et al. (2009). Koesterke et al. (1991) and Crowther \& Smith (1997) derived nearly equal stellar parameters, including 
a stellar temperature of about $T_{*}=35 \mathrm{kK}$. HK2000 obtained a stellar temperature of $T_{*}=39.8 \mathrm{kK}$. Our state of the art atmosphere models, however, need a temperature of $T_{*}=47 \mathrm{kK}$ to reproduce the observed $\mathrm{He} \mathrm{I} / \mathrm{He}$ II line ratios. These differences are attributable to the line-blanketing effect included in our models. The higher stellar temperature contributes to an increase in the derived luminosity of about 0.5 dex compared to Koesterke et al. (1991), Crowther \& Smith (1997), and HK2000. The derived mass-loss rate, on the other hand, is fairly the same.

Bonanos et al. (2009) find an infrared excess for this object by comparing a $T_{*}=45 \mathrm{kK}$ CMFGEN model from Smith et al. (2002) with the observed SED. However, our fit does not show a clear infrared excess, although a slight mismatch of the infrared photometry can be seen in Fig. C.23. We note that this discrepancy may be attributed to the IUE spectra, which are of poor quality (bad S/N, arbitrary continuum shape of the IUE longwavelength spectrum).

BAT99 59 is listed as WN4o?+B binary candidate in the BAT99 catalog. Foellmi et al. (2003b) found a period of $4.7 \mathrm{~d}$, reclassified the primary to $\mathrm{WN} 4 \mathrm{~b}$ and specified the companion to be an O8: star. They still assign a question mark to the binary status because the determined radial velocity amplitude is close to their detection limit. Since distinct absorption lines of the companion are visible in the spectra, the companion should contribute substantially to the total flux. The derived stellar properties (Table 2) are thus to be considered with caution. Koesterke et al. (1991) used pure helium models to derive lower limits for the stellar temperature and luminosity than our more sophisticated models.

BAT9960 is listed as WN3+OB binary candidate in the BAT99 catalog, but Foellmi et al. (2003b) did not find a radial velocity period and reclassified the object to WN4(h)a. Furthermore, these authors identified the absorption lines visible in their spectra to be intrinsic to the wind of the WN star. Therefore, we consider this star to be single for the time being. Unfortunately, we do not have UV spectra of this star, which had never been analyzed with stellar atmosphere models before. We confirm the presence of hydrogen $\left(X_{\mathrm{H}}=0.2\right)$ and thus the above classification.

BAT99 62 was classified as WN3(h) by Foellmi et al. (2003b) due to indications of hydrogen in its spectrum. Our analysis is inconclusive at this point. A reasonable fit can be achieved with a hydrogen-free model as well as with a model of a moderate hydrogen mass-fraction of $X_{\mathrm{H}}=0.1$. All optical spectra at hand exhibit distinct absorption lines in place of the O III-nebular emission lines at $4959 \AA$ and $5007 \AA$, likely caused by an over correction of the diffuse emission. If this is true, the Balmer series will probably be narrowed by the inadequate nebular subtraction, lending credence to the relatively high hydrogen abundance derived in the line fit. In this paper, we present for the first time stellar parameters for this star, derived by spectral analysis. Regrettably, no UV spectra are available for this star.

BAT9963 is listed as a binary candidate in the BAT99 catalog on the basis of absorption lines possibly belonging to a companion star. However, no binary period was found by the radial velocity analysis of Foellmi et al. (2003b). Moreover, Cowley et al. (1984) and Foellmi et al. (2003b) argue that the absorption components are intrinsic to the WR wind. Thus, the object was classified as WN4ha: by Foellmi et al. (2003b). We treat this star as single until its binary status is confirmed. Cowley et al. (1984) note that the high radial velocity suggests that the star is a runaway. This conclusion is confirmed by the radial velocity study of Schnurr et al. (2008).

A lower limit for the stellar temperature was derived by Koesterke et al. (1991) on the basis of the helium spectrum. More elaborate models are used by HK2000, deriving a stellar temperature of $T_{*}=70.8 \mathrm{kK}$. In comparison to this work, our best fitting model with $T_{*}=63 \mathrm{kK}$ is one grid step cooler. This lower temperature is justified by a slightly better fit of the nitrogen lines. Apart from that, we obtained similar values for the mass-loss rate, while the luminosity and hydrogen abundance are slightly lower.

The filamentary ring nebula associated with BAT99 63 was studied by Nazé et al. (2003b). As no He II nebular emission is detected by these authors, they obtained an upper limit for the number of He II ionizing photons delivered by the exciting star, which amounts to $<2.5 \times 10^{45} \mathrm{He}$ II ionizing photons per second. This agrees with our final model, which does not predicts a significant number of He II ionizing photons (see Table A.3).

BAT9964 was listed as WN4+OB? binary candidate in the BAT99 catalog. A period of $37.6 \mathrm{~d}$ was found by Foellmi et al. (2003b), who classified the companion as O9 and specified the primary to be a WN4o star. However, we expect that the companion does not contribute much to the bolometric luminosity.

BAT99 65 was classified as WN4o by Foellmi et al. (2003b). For this star we only have a co-added optical spectrum, which is not flux-calibrated so that the observed SED is covered by photometry alone. However, the coverage is complete from UV to mid-infrared.

BAT9966 is a WN3(h) star (Foellmi et al. 2003b) for which stellar parameters have been presented for the first time in this paper. Unfortunately, no UV spectra are available for this star.

BAT9967 is listed as a binary candidate with a WN5o?+OB classification in the BAT99 catalog, though Foellmi et al. (2003b) concluded from their radial velocity study that this star is probably not a short-period binary. Foellmi et al. (2003b) note that the absorption features visible in their spectra are strongly blue-shifted. They emphasize that if this shift arises from a companion, it would result in distinct radial velocity variations that are not observed. Hence, these authors argue that the absorption lines are intrinsically formed in the wind of the WN star, and accordingly reclassify the object to WN5ha. Nevertheless, we still treat this star as a binary suspect because of the X-ray emission detected by Guerrero \& Chu (2008a).

We derived a stellar temperature of $T_{*}=47 \mathrm{kK}$, only slightly higher than the value obtained by HK2000 $\left(T_{*}=44.7 \mathrm{kK}\right)$. The luminosity and mass-loss rate derived in our analysis are slightly lower compared to the result obtained by HK2000, while the hydrogen abundance is 0.1 dex higher.

BAT99 68 is a transition type O3.5 If*/WN7 star (Crowther \& Walborn 2011). The physical parameters were previously estimated with FASTWIND by Massey et al. (2005), but the authors note that these values are only poorly constrained. Our new analysis results in a stellar temperature of $T_{\mathrm{eff}}=44 \mathrm{kK}$, 
marginally higher than the temperature estimate obtained by Massey et al. (2005, $\left.T_{\text {eff }}=40-42 \mathrm{kK}\right)$. The mass-loss rate derived in our new analysis is a factor of three lower, even if we account for the impact of different terminal velocities and density contrasts. We note that a mass-loss rate as high as obtained by Massey et al. (2005) results in considerably too strong emission lines in our synthetic spectra.

The IUE spectra associated with this object are noisy and do not show WR features. Therefore, we precluded these spectra from our analysis. We also excluded the optical spectrum measured by Torres-Dodgen \& Massey (1988), since the continuum slope of this flux-calibrated spectrum disagrees with the optical photometry (BAT99) and the HST spectra. Moreover, the available infrared photometry (Cutri et al. 2012; Kato et al. 2007) is inconsistent and cannot be fitted in accordance with the UV and optical data. The infrared observations probably suffer from contributions of nearby objects, which are visible on WFPC2 images within $1^{\prime \prime}$ around BAT99 68. Fitting the UV and optical data, we derived a luminosity of $\log \left(L / L_{\odot}\right)=6.0$ which is a factor of two lower than previously obtained by Massey et al. (2005).

BAT99 71 was identified as a short-period binary by Foellmi et al. (2003b). They classified the primary as WN4 and specified the companion to be a O8: star. Its IUE spectrum is not usable: it is noisy and does not have distinct WR features. Therefore, we reject these spectra from our analysis.

BAT9972 is probably a medium-period binary according to Foellmi et al. (2003b), assigning an O3: type to the companion and reclassifying the primary to WN4h. Foellmi et al. (2003b) mention that an absorption-line component is clearly visible in the spectrum. However, we fit the star as a single WN star and achieve a good fit for almost the whole spectrum. For that reason, and due to the well-reproduced SED, we expect that the companion does not contribute much to the bolometric luminosity. We note that the luminosity obtained in our analysis is derived from visual narrowband, 2MASS and IRAC photometry alone since no flux-calibrated spectra were available.

BAT99 73 was classified as WN5ha by Crowther \& Hadfield (2006). Unfortunately, the spectrum from Foellmi et al. (2003b) is the only one available to us. Since this spectrum is not fluxcalibrated, the luminosity is derived from photometry alone, but the available photometry covers the whole range from the UV to mid-infrared so that the luminosity is nevertheless well constrained. This WN star was not analyzed by means of stellar atmosphere models before.

BAT99 74 is a WN3(h)a star (Foellmi et al. 2003b), which is analyzed in this work for the first time. The spectrum of this star is characterized by small absorption lines and moderate emission lines. Foellmi et al. (2003b) identified the absorption lines to be intrinsic to the wind of the WN star. Both absorption and emission lines can be accordantly reproduced with our singlestar model. From the $\mathrm{H} \alpha$ and $\mathrm{H} \beta$ lines, we estimated a hydrogen mass-fraction of $X_{\mathrm{H}}=0.2$, which underpins the $\mathrm{WN}(\mathrm{h})$ classification. No flux-calibrated spectra are available for BAT9974. However, the luminosity is well constrained due to the photometric coverage of the SED from UV to mid-infrared.

BAT99 75 was classified as WN4o by Foellmi et al. (2003b). The prominent emission lines in the spectra of this star are best reproduced with a hydrogen-free model. For the first time, we present here parameters for this star.

BAT99 76 is a WN9ha star (Schnurr et al. 2008), which has been analyzed by Koesterke et al. (1991), Crowther et al. (1995b), Pasquali et al. (1997), and HK2000. Two UV spectra and three optical spectra are at hand for the analysis of this star (see Table A.1 and A.2). All can be fitted with the same model, which give us confidence in the derived physical parameters.

The stellar temperature derived in our analysis, $T_{*}=35 \mathrm{kK}$, fits in the range of temperatures $T_{*}=30-38.7 \mathrm{kK}$ obtained by the authors mention above. The mass-loss rates presented in these papers are nearly the same with the exception of the results obtained by HK2000, who inferred a mass-loss rate a factor of two higher. Our analysis confirms the lower mass-loss rates obtained by Koesterke et al. (1991), Crowther et al. (1995b), and Pasquali et al. (1997). The luminosity derived in the previous papers range from $\log \left(L / L_{\odot}\right)=5.4$ to $\log \left(L / L_{\odot}\right)=5.8$, which in principle agrees with the value $\left(\log \left(L / L_{\odot}\right)=5.66\right)$ derived in this work. The hydrogen abundance provided in Table 2 is lower than previously derived by Crowther et al. (1995b), Pasquali et al. (1997), and HK2000.

BAT99 77 was revealed as SB1 binary with a period of $3 \mathrm{~d}$ by Moffat (1989). This finding was later confirmed by the radial velocity study of Schnurr et al. (2008). These authors classified the object as WN7ha. Since no spectral features can be unambiguously attributed to the companion, the contribution of the companion to the overall flux is hard to estimate. Thus, the physical parameters listed in Table 2 need to be taken with caution. A tentative detection of X-ray emission has been reported by Guerrero \& Chu (2008a).

BAT99 78 is located in a tight cluster in the western part of LH90 with several additional sources within a radius of less than 1" (Walborn et al. 1999). This star is listed as WN4 in the BAT99 catalog, whereas Foellmi et al. (2003b) assign a WN6 spectral type to it. According to these authors, the spectrum is a superposition of the WN star and nearby objects. This is attributable to the crowded environment and the relatively wide slit width used by these authors. The co-added spectrum observed by Foellmi et al. (2003b) resembles an Of supergiant rather than a WN star since it shows distinct Of features and a $\mathrm{H} \beta$ line clearly in absorption (Crowther \& Walborn 2011). A reasonable fit of this spectrum can be achieved with a model of $T_{*}=45 \mathrm{kK}$.

Beside the spectrum obtained by Foellmi et al. (2003b) we retrieved a relatively short optical spectrum from the HST archive which exhibits a clear WN characteristic. This spectrum can be well fitted with a model corresponding to a stellar temperature of $T_{*}=71 \mathrm{kK}$. Since the HST spectrum is flux-calibrated, it can also be used for the SED fit. This results in an implausibly small luminosity of $\log \left(L / L_{\odot}\right)=4.64$. The flux of the HST spectrum disagrees with the available photometry, which in turn is not uniform. For example, the data of the $J$-band magnitude varies from 11.6-14.68 mag. A fit of the 2MASS, Spitzer IRAC, and WISE photometry results in a luminosity of $\log \left(L / L_{\odot}\right)=6.8$. The observational discrepancies might be traced back to the small angular distance of BAT9978 to the other cluster members (see, e.g., Walborn et al. 1999). The smallest WISE aperture covers not only the four closest objects to BAT9978, but rather a large part of the whole cluster around it. 
Walborn et al. (1999) were able to chiefly resolve the host cluster of BAT9978 with the Wide Field/Planetary Camera 1 (WFPC1) aboard the HST. They obtained visual broadband photometry, which we adjusted to account for the contribution of the emission lines, using the correction factor derived by Breysacher (1986). This visual magnitude $M_{v}=14.84$ results in a luminosity of $\log \left(L / L_{\odot}\right)=5.7$, assuming that the continuum slope of the HST spectrum and the thereof derived color excess of $E_{b-v}=0.2$ is correct.

Due to the X-ray emission detected by Guerrero \& Chu (2008a), we treat this object as a binary candidate, although Foellmi et al. (2003b) find no significant periodicity in their radial velocity study, which is based on a small number of observations of this object.

BAT99 79 is listed as WN7h+OB binary candidate in the BAT99 catalog. However, Schnurr et al. (2008) do not find periodic radial velocity variations. Despite this nondetection, we still regard this object as a binary candidate since a considerable amount of X-ray emission has been detected by Guerrero \& Chu (2008a). We note that Crowther \& Smith (1997) estimated a significant contribution of the companion to the overall flux by means of their own spectra. In contrast, we expect that the companion does not contribute much to the bolometric luminosity, since only very small absorption features can be seen superimposed on the emission lines of the WN star in the spectrum observed by Schnurr et al. (2008). Therefore, the stellar parameters listed in Table 2 should represent a reliable approximation.

BAT9980 is listed as O4 If/WN6 transition type star in BAT99 catalog. Schnurr et al. (2008) reclassified the star to WN5h:a, arguing that the spectrum shows a diluted WN star rather than a hot slash star. However, no significant radial velocity variations were detected by Schnurr et al. (2008). Nevertheless, we treat this object as a binary suspect because of the X-ray emission reported in Guerrero \& Chu (2008a).

The only spectrum available to us is characterized by absorption and moderate emission lines. Although it might show a binary, both absorption and emission lines can be accordingly reproduced with our single-star model.

BAT9981 was classified as WN5h by Foellmi et al. (2003b). The IUE long-wavelength spectrum of this star apparently has a considerably lower flux than the IUE short-wavelength spectrum. The former does not fit to the rest of the observed SED. Therefore, it is not further considered in our analysis of BAT99 81, which provides stellar and wind parameters of this object for the first time. Foellmi et al. (2003b) highlights the differences between the results of Cowley et al. (1984) and their own radial velocity study, which exhibits no clear radial-velocity deviation from the mean $v_{\text {rad }}$ of their WN sample, whereas Cowley et al. (1984) argued in favor of a runaway nature.

BAT9982 is a WN3b star (BAT99), for which the detection of X-rays has been reported by Guerrero \& Chu (2008a). We treat this star as a binary on the basis of the X-ray emission. However, no periodic radial velocity variations were found by Foellmi et al. (2003b). The IUE spectrum assigned to BAT99 82 was taken $1.2^{\prime}$ away from its position and does show some helium emission, but no nitrogen. Furthermore, its flux is not compatible to the SED composed of the optical spectrum by Torres-Dodgen \& Massey (1988) and the photometry from the 2MASS catalog, so that the IUE spectrum was ignored.
BAT99 86 has recently been classified as WN3(h) by Doran et al. (2013). Unfortunately, no UV spectrum is available for this object. The hydrogen abundance is hard to determine because of absorption features superimposed on the Balmer lines. The best fit is obtained with a hydrogen-free model. However, we note that the emission lines without the absorption components might agree with an hydrogen mass-fraction of $X_{\mathrm{H}}=0.1$. On the contrary, Doran et al. (2013) derived a hydrogen abundance of $X_{\mathrm{H}}=0.2$ on the basis of their stellar atmosphere models. However, a hydrogen abundance this high would result in a considerable overprediction of the Balmer lines by our synthetic spectra. Apart from that, Doran et al. (2013) derived a stellar temperature that is $8 \mathrm{kK}$ higher and a luminosity that is $0.1 \mathrm{dex}$ higher than our values. However, the strongest deviation to the results presented by Doran et al. (2013) is obtained in the derived mass-loss rate, which is more than nine times lower in our analysis. Our models with a mass-loss rate as high as derived by Doran et al. (2013) considerably overpredict the equivalent width of all emission lines.

BAT99 88 is the second WNE/WCE transition type star in the LMC. These stars exhibit a clear carbon enrichment compared to the rest of the LMC WN stars. The best fit is obtained with a carbon mass-fraction of $X_{\mathrm{C}}=0.005$ in accordance with the estimate by Doran et al. (2013).

This is one of the hottest stars in our sample, with a stellar temperature of $T_{*}=112 \mathrm{kK}$. This value is about $30 \mathrm{kK}$ higher than the stellar temperature $\left(T_{*}=80 \mathrm{kK}\right)$ obtained by Doran et al. (2013). In contrast, our model with $T_{*}=80 \mathrm{kK}$ underpredicts the equivalent width of the He II $\lambda 4686$ line with respect to the other He II lines, while the model with $T_{*}=112 \mathrm{kK}$ is able to simultaneously reproduce the equivalent width of all He II lines. Furthermore, the N IV and N V lines are slightly better reproduced at this higher temperature. However, we note that this star is located within the regime of parameter degeneracy (cf. Sect. 4.2).

Contrary to the stellar temperature, the luminosity and the mass-loss rate derived in this work are comparable to the values derived by Doran et al. (2013). Unfortunately, we neither have UV spectra nor flux-calibrated spectra. Thus, the luminosity is derived from UV, optical, near- and mid-infrared photometry alone.

The emission lines of this object exhibit round profiles. Thus, this star falls in the category of the so-called round line stars (cf. comment on BAT99 7). A reasonable fit of the observed line profile is achieved by convolving our synthetic spectrum with a rotation profile corresponding to a rotational velocity of $v_{\text {rot }} \sin i=1200 \mathrm{~km} \mathrm{~s}^{-1}$.

BAT99 89 is a WN7h star (BAT99), which was previously studied by Crowther \& Smith (1997) and Doran et al. (2013). The best fit is achieved at a stellar temperature of $T_{*}=50 \mathrm{kK}$, which agrees well with the recent results of Doran et al. (2013). In contrast, Crowther \& Smith (1997) obtained a stellar temperature that is $10 \mathrm{kK}$ lower. However this value is excluded by our analysis, since the synthetic spectra clearly overpredicts the He I/He II ratio at this temperature. This difference in the stellar temperature can be attributed to the line-blanketing effect, which is included in modern atmosphere models, but was not regarded at the time of the study by Crowther \& Smith (1997). The luminosity derived in this paper $\left(\log \left(L / L_{\odot}\right)=5.56\right)$ is only marginally higher than the value obtained by Doran et al. (2013), while Crowther \& Smith (1997) derived a luminosity which is a factor 
of two lower. In terms of the mass-loss rate, we obtain a value of almost $50 \%$ lower compared to the recent results by Doran et al. (2013), whereas the mass-loss rate derived by Crowther \& Smith (1997) is slightly higher.

BAT9991 was resolved into multiple components by Testor et al. (1988) and later by the HST observations of Walborn et al. (1995). The WN star has been classified as WN6(h) by Evans et al. (2011), although their ground-based observations were not able to entirely resolve the components of this object. The same seems to hold for the spectrum obtained by Schnurr et al. (2008), which does not resemble the HST spectrum for BAT9991 obtained by Walborn et al. (1995). For that reason, our spectroscopic analysis relies on the HST spectrum alone, which unfortunately covers only $1500 \AA$ out of $3300-4800 \AA$. Since this range comprises neither the $\mathrm{H} \alpha$ nor $\mathrm{H} \beta$, the determination of the hydrogen abundance is based on the higher members of the Balmer series alone. With a hydrogen mass-fraction of $X_{\mathrm{H}}=0.2$, we confirm the corresponding classification. In the SED fit, we exclusively used the HST photometry from Walborn et al. (1995) and the HST flux-calibrated spectra from Walborn et al. (1999), since these observations are distinguished by their particularly high spatial resolution, which seems to be necessary for reliable results in this tight cluster. This star had never been analyzed before.

BAT9992 was classified as $\mathrm{WN} 3: \mathrm{b}(+\mathrm{O})+\mathrm{B} 1 \mathrm{Ia}$ by Schnurr et al. (2008). Both the BAT99 catalog and Schnurr et al. (2008) give a binary period of $4.3 \mathrm{~d}$ for this star. We note that the detection of X-ray emission has been reported by Guerrero \& Chu (2008a). Although the optical spectra seem to be considerably affected by the companions, they still allow us to assess parameters like the stellar temperature. Furthermore, this object belongs to the round-line stars, since the emission lines exhibit a round shape which can only be reproduced with a rotational velocity of $v_{\text {rot }}=1500 \mathrm{~km} \mathrm{~s}^{-1}$. Another interesting fact is the significant strength of the C IV $\lambda 5808$ emission line, suggesting that either the system comprises an additional WC star or that the WN star belongs to the rare WNE/WCE transition type. The SED can be well reproduced with a single-star model.

BAT99 93 is one of the stars listed as WN stars in the BAT99 catalog which has been downgraded to O3 If* by Evans et al. (2011) and Crowther \& Walborn (2011). Tentative X-ray emission has been detected by Guerrero \& Chu (2008a). Thus, we treat this object as a binary suspect, although no radial velocity variations have been detected by Schnurr et al. (2008). The only optical spectrum at hand lacks a subtraction of the diffuse background and shows only a truncated $\mathrm{H} \beta$ line. For these reasons, we are not able to give a precise hydrogen abundances for this star.

BAT99 94 is characterized by broad emission lines with a round line shape. Therefore, this WN4b star (BAT99) is classified as a round line star (cf. comment on BAT99 7). The round shape of the emission lines requires a convolution of the model spectrum with a rotation profile corresponding to a rotational velocity of $v_{\mathrm{rot}} \cdot \sin i=1600 \mathrm{~km} \mathrm{~s}^{-1}$.

Unfortunately, we do not have UV spectra for this star. In this work, we derive a stellar temperature of $T_{*}=141 \mathrm{kK}$, which is significantly higher compared to the previous results by HK2000 $\left(T_{*}=100 \mathrm{kK}\right)$. In our analysis, the model with the higher temperature results in a sightly better fit of the He II $\lambda 4686$,
C IV $\lambda 5808$ and N IV $\lambda 4060$ lines. However, we note that star is located in the regime of parameter degeneracy (cf. Sect. 4.2). In comparison with HK2000, we have obtained a factor of two lower mass-loss rate, while the luminosity is a factor of three higher. The higher luminosity originates from the higher temperature and thus higher bolometric correction.

BAT9995 was identified as binary by Schnurr et al. (2008). These authors find radial velocity variations with a period of $2.1 \mathrm{~d}$. Evans et al. (2011) classified the object as WN7h+OB. We expect that the companion does not contribute much to the bolometric luminosity, since we do not see spectral features of the companion in any of the available spectra. However, all optical spectra available to us suffer from either an oversubtraction of the diffuse background, or even a missing background subtraction. For this reason we cannot give a reliable value for the hydrogen content in the atmosphere of this star.

In comparison to the previous analysis by Crowther \& Smith (1997), our best fit is achieved at a $14 \mathrm{kK}$ higher stellar temperature of $T_{*}=50 \mathrm{kK}$. The temperature derived by Crowther \& Smith (1997), however, would result in an overprediction of the He I $\lambda 5877$ to He II $\lambda 5412$ line ratio. The different temperatures likely arise from the line blanketing, which is incorporated in our stellar atmosphere models, but was not accounted for in the models used by Crowther \& Smith (1997). The higher temperature derived in this work results in a luminosity that is almost a factor of three higher compared to the previous results by Crowther $\&$ Smith (1997). The mass-loss rate derived in this work, on the other hand, is identical to the value given by Crowther \& Smith (1997).

BAT99 96 is of subtype WN8 (Schnurr et al. 2008) and located in the southern part of 30 Doradus. We do not have fluxcalibrated spectra or intrinsic narrowband photometry for this star, which renders it difficult to obtain precise values for the stellar luminosity and the interstellar reddening. Moreover, the available UBVR photometry is inconsistent since the values derived by various authors differ by up to $2 \mathrm{mag}$. The narrowband photometry listed in the BAT99 catalog is derived from visual broadband photometry (Parker 1993, $V=13.65 \mathrm{mag}$ ) by means of the correction factor found by Breysacher (1986). The visual magnitude obtained by Parker (1993) is slightly higher than the value ( $V=13.76 \mathrm{mag}$ ) observed by Selman et al. (1999). Massey (2002) and Zaritsky et al. (2004) obtained higher magnitudes of $V=12.84 \mathrm{mag}$ and $V=12.9 \mathrm{mag}$, respectively. In contrast, Duflot (2010) and Girard et al. (2011) derived lower visual magnitudes of $V=14.5 \mathrm{mag}$ and $14.47 \mathrm{mag}$, respectively.

To construct the SED, we relied on the optical photometry obtained by Parker (1993), 2MASS and IRAC photometry (Bonanos et al. 2009). Similar to BAT99 98, the SED fit results in a relatively high value for the color excess $\left(E_{b-v}=0.7 \mathrm{mag}\right)$ and the luminosity $\left(\log \left(L / L_{\odot}\right)=6.4\right)$. In contrast to our results, Doran et al. (2013) derived a color excess of $E_{B-V}=0.65 \mathrm{mag}$, which corresponds to $E_{b-v}=0.54 \mathrm{mag}$. We cannot achieve a reasonable SED fit with a color excess as low as derived by these authors. Moreover, we obtain the same color excess and luminosity in our SED fit regardless of whether the optical broadband photometry from Parker (1993) or the optical narrowband magnitudes $(v=13.82 \mathrm{mag}, b-v=0.49 \mathrm{mag})$ given by Doran et al. (2013) are used.

The stellar luminosity derived in our analysis is much higher compared to the results derived by Crowther \& Smith (1997) and Doran et al. (2013). These authors determined a stellar luminosity of $\log \left(L / L_{\odot}\right)=5.86$ and $\log \left(L / L_{\odot}\right)=6.04$, respec- 
tively. In comparison to Doran et al. (2013), the deviation in the luminosity primarily originates from the different reddening parameters. The additional IRAC photometry incorporated in our analysis enhances the constraints on the shape of the SED (see Fig C.38), which gives us confidence in the derived luminosity and color excess. Apart from these two parameters, we achieve a good agreement for the stellar temperature and the mass-loss rate obtained in this work and the values presented by Doran et al. (2013). Due to the missing subtraction of the diffuse background in the spectra obtained by Schnurr et al. (2008), we note the doubtful hydrogen abundance determined in our analysis.

We consider this star to be single, since neither periodic radial-velocity variations nor X-ray emission have been detected by Schnurr et al. (2008) and Guerrero \& Chu (2008a,b), respectively. However, Parker (1993) argued in favor of a multiple object on the basis of their ground-based photometry. However, we cannot detect any visual companion either on the images taken with the Visual and Infrared Telescope for Astronomy (VISTA) for the VISTA survey of the Magellanic Clouds system (VMC; Emerson et al. 2006; Dalton et al. 2006) or on the high resolution images with the WFC3 aboard the HST (O'Connell 2008). From a comparison of the empirical HRD position (see Figs. 7 and 10) with the stellar evolution tracks calculated by Meynet \& Maeder (2005), we estimate an initial mass on the order of $M_{\text {init }}=100 M_{\odot}$. Thus, we consider this object to belong to the category of very massive stars.

BAT9997 is another transition type O3.5 If*/WN7 star (Crowther \& Walborn 2011; Evans et al. 2011). Unfortunately, the only spectrum available to us is affected by nebular emission due to a missing background subtraction. Thus, we are not able to give a precise hydrogen abundances for this star. We derive a stellar temperature that is slightly higher compared to the results published by Doran et al. (2013), while the mass-loss rate and the luminosity are higher by about 0.15 dex.

BAT99 98 is a WN6 star (Schnurr et al. 2008) located near R136. The derived luminosity, and thus the stellar mass as well, are comparable to those of the very massive stars in the core of R136 analyzed by Crowther et al. (2010). This star is distinguished by the relatively high extinction of $E_{b-v}=0.8$ mag derived from the SED fit. Unfortunately, no flux-calibrated spectra and intrinsic narrowband photometry (Smith system) are available. Broadband photometry (e.g., Johnson system), on the other hand, is contaminated by the prominent emission features.

The optical narrowband photometry from the BAT99 catalog is a corrected Johnson $V$ magnitude from Parker (1993), using the subtype-dependent correction factor derived by Breysacher (1986). Following this procedure, we obtain a narrowband magnitude of $v=13.70 \mathrm{mag}$. Further broadband photometry is available from Selman et al. (1999) and Massey (2002), whereas the coordinates quoted by Massey (2002) show the largest deviation from the position stated in Simbad. The $V$ band magnitudes obtained by these authors, corrected for the contribution of the emission lines, result in $v=13.64 \mathrm{mag}, v=13.61 \mathrm{mag}$ and $v=13.67 \mathrm{mag}$, respectively. The $v$ magnitude inferred by Breysacher (1986) is a corrected Strömgen $y$ magnitude that amounts to $v=13.65 \mathrm{mag}$. The optical photometry, together with the near- and mid-infrared photometry, gives rise to the high color excess and a stellar luminosity of $\log \left(L / L_{\odot}\right)=6.7$. However, the luminosity has a relatively large uncertainty, since it is derived from photometry alone.
So far, neither periodical radial velocity variations (Schnurr et al. 2008) nor X-ray emission (Guerrero \& Chu 2008a,b) were detected. Thus, we treat this object as a single star, although the moderate fit quality may indicate a line dilution due to a yet undetected companion, as already suggested by Crowther \& Smith (1997). The applied model underpredicts the He II $\lambda$ 5201, He I $\lambda 4471$ lines and overpredicts the He II $\lambda 4686$ line. An adjustment of the temperature in one direction or the other spoils the fit of either the N IV $\lambda 4060$ line or the N III $\lambda 4640$ line, used as main diagnostic lines for this object.

Unfortunately, the only spectrum available to us is compromised by a missing subtraction of the diffuse background. This is probably the reason that the spectrum is affected by nebular contamination, a fact that entails an uncertain determination of the hydrogen content. This is aggravated by the fact that $\mathrm{H} \beta$ and $\mathrm{H} \gamma$ are truncated (see Schnurr et al. 2008).

The luminosity derived in this work corresponds to a current mass of $M_{*}=226 M_{\odot}$, according to the mass-luminosity relation from Gräfener et al. (2011). However, the error margin of this quantity is large, since it is calculated from the luminosity and the hydrogen abundance, which are in turn affected by considerable uncertainties. By comparing the empirical HRD position to stellar evolution tracks calculated by Yusof et al. (2013), we estimated an initial mass of at least $M_{\text {init }}=250 M_{\odot}$. Thus, this star is one of the most massive stars hitherto known in the LMC.

BAT99 99 is a transition type O2.5 If*/WN6 star. Two spectra are used for the analysis of this star, an HST spectrum and a spectrum obtained by Schnurr et al. (2008). Both spectra are characterized by small absorption lines and relatively weak emission lines. We note that the spectrum taken by Schnurr et al. (2008) shows a substantially weaker He II $\lambda 4686$ line, although the N IV $\lambda 4060$ line is of comparable strength in both observations. However, we do not consider the ground-based spectrum observed by Schnurr et al. (2008) in our analysis because it seems to be contaminated with nebular emission and the $\mathrm{H} \beta$ line is arbitrary truncated.

The HST spectra exhibit a flux of roughly a factor of two lower in comparison to the photometric data listed in the BAT99 catalog and the infrared photometry by Kato et al. (2007). Since BAT99 99 is located in the vicinity of the 30 Doradus core, this mismatch can be attributable to this crowded environment. Therefore, we have derived the luminosity from the HST spectra alone, which are distinguished by the high spatial resolution of the HST. The infrared excess presented in the SED fit in Fig. C.39 presumably originates from nearby sources visible on high resolution HST images.

The detection of X-ray emission has been reported by Guerrero \& Chu (2008a), which is indirect evidence for the binary nature of this object (see Sect.2.2). Direct evidence has been supplied by Schnurr et al. (2008). These authors have found radial velocity variations, corresponding to a period of $93 \mathrm{~d}$. We did not detect spectral lines of a companion star in either of the spectra used in our analysis. Therefore, the contribution of an OB companion to the overall flux cannot be properly evaluated. Thus, the parameters for the WN component listed in Table 2 might be affected by this unknown flux contribution.

BAT99 100 is a WN7 star located in the crowded environment close to the core of 30 Doradus. In the optical spectral range, we used two spectra, an archival HST spectrum and a spectrum obtained by Schnurr et al. (2008). The latter shows strong nebular emission lines, but only small stellar emission lines, whereas the former exhibit much stronger emission lines. Due to the limited 
spatial resolution of the ground-based observations, our analysis is mainly based on the HST spectrum.

In a former study, Crowther \& Smith (1997) analyzed this star with unblanketed stellar atmospheres. In contrast to their work, we achieve the best fit with a model corresponding to a stellar temperature of $T_{*}=47 \mathrm{kK}$, which is $15 \mathrm{kK}$ higher than the temperature derived by these authors. At stellar temperatures below $47 \mathrm{kK}$, our models overpredict the $\mathrm{He} \mathrm{I} / \mathrm{He}$ II line ratio. These differences can be attributed to the line-blanketing effect. The luminosity derived in this work is a factor of two higher compared to the results obtained by Crowther \& Smith (1997), which is attributable to the different bolometric correction due to the higher stellar temperature. The mass-loss rate is nearly the same compared to the previous estimate by Crowther $\&$ Smith (1997). Since the HST spectrum does not cover the $\mathrm{H} \alpha$ and $\mathrm{H} \beta$ lines, the hydrogen abundance is derived from the higher Balmer members alone.

BAT99 100 was found by Guerrero \& Chu (2008a) to show $\mathrm{X}$-ray emission. Thus, we consider this object to be a binary suspect, although no radial velocity variations were discovered by Schnurr et al. (2008). The noteworthy infrared excess of this object might be attributed to a hidden companion.

BAT99 102 was classified as WN6 by Schnurr et al. (2008). According to these authors, the spectra are contaminated by the flux of the WC star BAT99 101, since it was not possible to resolve these close objects even under the best seeing conditions (Schnurr et al. 2008). Unfortunately, this is the only spectrum available to us. Since this spectrum lacks a sufficient background subtraction, we are not able to derive a reliable hydrogen abundance. Moreover, the flux contribution of BAT99 101 to the total flux is unknown but not negligible, since the broad line wings of the He II $\lambda 4686$ line and He II $\lambda 5412$ line (Fig. C.40) probably originate from the WC star. Therefore, the physical parameters listed in Table 2 need to be taken with caution.

According to Guerrero \& Chu (2008a), BAT99 101, together with BAT99 102 is one of the brightest X-ray source in 30 Doradus. Unfortunately, the Chandra ACIS instrument is not able to resolve BAT99 101 and 102, so that the X-ray emission cannot be attributed to one of these stars alone. The ROSAT HRI observation analyzed by Guerrero \& Chu (2008b) results in the same conclusion, even though BAT99 101-103 could not be resolved into individual objects by ROSAT. Moffat et al. (1987) found radial velocity variations with a period of $2.76 \mathrm{~d}$ for BAT99 102, whereas Schnurr et al. (2008) found the same period for the nearby BAT99 103 instead of for BAT99 102. Until the binary status is confirmed, we consider this star as a binary suspect.

BAT99 103 is a WN5(h)+O binary (Evans et al. 2011) located in the direct neighborhood of BAT99 101 and 102. This star was identified as a binary with a period of $2.76 \mathrm{~d}$ by Schnurr et al. (2008). Tentative X-ray emission was reported by Guerrero \& Chu (2008a).

Unfortunately, no background subtraction was applied to the spectrum shown in Fig. C.41. Since this is the only spectrum available to us, we are not able to derive a meaningful hydrogen abundance for this object.

BAT99 104 is a O2 If*/WN5 transition type star located close to the center of 30 Doradus. We have three optical spectra at hand, two archival HST spectra (H $\alpha$ and 3200-4800 $\mathrm{A}$ ) and one spectrum obtained by Schnurr et al. (2008). Considerable differences can be detected between these data. For example, the HST spectrum exhibits significantly higher emission-line strengths of the He II $\lambda 4686$ and the N IV $\lambda 4060$ lines. These observational discrepancies might result from a nearby source that contaminated these observations. Due to the higher spatial resolution of the HST compared to the ground-based telescopes, we rely primarily on HST data.

The optical narrowband magnitude listed in the BAT99 catalog is in excellent agreement with the HST spectrum, which covers the spectral range from $3200 \AA$ to $4800 \AA$. Another HST spectrum covering $\mathrm{H} \alpha$, on the other hand, exhibits a flux of approximately 0.1 dex higher. Thus, the uncertainty in the derived luminosity (Table 2) is higher than for the other stars in our sample.

For the infrared part of the SED, we used the photometric data obtained by Kato et al. (2007), instead of the low quality 2MASS data. With the exception of the $J$-band magnitude, the photometric data measured by Kato et al. (2007) appear to be unaffected by nearby sources. However, in comparison to the optical data, we find an infrared excess that might be caused by nearby sources, a hidden companion, or dust emission.

BAT99 105 is listed as a transition-type star in the BAT99 catalog, but has been demoted to O2 If* by Crowther \& Walborn (2011). We have UV as well as optical spectra at hand for this star. The optical observations (an archival HST spectrum and a ground-based spectrum observed by Schnurr et al. 2008) possibly show two different objects. For example, the HST spectrum exhibits the He II $\lambda$ 4201, 4542 lines in absorption, whereas these lines are in emission in the spectrum obtained by Schnurr et al. (2008). Moreover, the He II $\lambda 4686$ and N III $\lambda 4640$ lines are appreciably stronger in the latter. Since the HST spectrum exhibits the same appearance as the UVES spectrum shown by Crowther \& Walborn (2011), we choose to rely on the HST data in the optical spectral range.

BAT99 105 is suspected to be a binary due to the X-ray emission detected by Guerrero \& Chu (2008a), although no significant radial velocity variations were detected by Schnurr et al. (2008). We note that the optical HST and the IUE shortwavelength spectrum can be consistently reproduced by the same single-star model.

This object was intensively analyzed by Heap et al. (1991), Pauldrach et al. (1994), de Koter et al. (1997), and Doran \& Crowther (2011). On the basis of modern stellar atmosphere models, Doran \& Crowther (2011) derived a stellar temperature of $T_{*}=49.8 \mathrm{kK}$, which agrees with our own results. In comparison to the latest comprehensive analysis by de Koter et al. (1997), our fit results in a $5 \mathrm{kK}$ higher stellar temperature, an identical luminosity, but a considerably lower mass-loss rate (a factor of 3.6 lower). Pauldrach et al. (1994) have derived a massloss rate of similar extent as de Koter et al. (1997). However, a mass-loss rate as high as derived by Pauldrach et al. (1994) and de Koter et al. (1997) results in a considerable overprediction of the emission lines. The studies of Pauldrach et al. (1994) and de Koter et al. (1997) were based on UV spectra alone.

BAT99 106 is a WN5h star (BAT99) located in the core of R 136. This star was studied by de Koter et al. (1997), Crowther \& Dessart (1998), and Crowther et al. (2010). Crowther et al. (2010) report it to be one of the most massive stars known so far. Our independent analysis basically confirms the physical parameters derived by Crowther et al. (2010). 
X-ray emission was detected by Guerrero \& Chu (2008a) for BAT99 106, 108, 109, and 110 with the Chandra satellite. Since these stars in the tight cluster R136 cannot be resolved by this instrument, we treat BAT99 106 as a single star, although the $\mathrm{X}$-ray emission might be associated with it and indicate colliding winds in a binary system.

We note that in addition to the photometry given in Sect. 2.3, we used $b$-band photometry (Crowther \& Dessart 1998) and $K_{\mathrm{S}}$-band photometry (Crowther et al. 2010) in the SED fit.

BAT99 107 has been identified by Taylor et al. (2011) as a massive SB2 binary system consisting of two O-type stars. Moffat (1989) found a radial velocity variation with a period of $52.7 \mathrm{~d}$. However, Schnurr et al. (2008) could not confirm this period.

BAT99 108 is the most massive star in the core of R 136 (Crowther et al. 2010). It is listed as WN5h star in the BAT99 catalog and has been analyzed by de Koter et al. (1997), Crowther \& Dessart (1998), and Crowther et al. (2010). In comparison to the most recent analysis by Crowther et al. (2010), we obtained fairly similar stellar parameters. Note that our SED fit (Fig. C.43) matches the HST spectra (UV and optical) consistently with the $K_{\mathrm{S}}$-band photometry (Crowther et al. 2010). We ignore the optical photometry (BAT99, Crowther \& Dessart 1998), which is inconsistent with the calibrated HST spectrum. X-ray emission is associated with BAT99 108 (cf. comment on BAT99 106).

BAT99 109 is another WN5h star (BAT99) in the core of R 136, previously analyzed by Crowther \& Dessart (1998) and Crowther et al. (2010). We have UV and optical HST spectra for this object. According to de Koter et al. (1997), the HST spectra of BAT99 109 are contaminated by the flux of BAT99 108 situated only $0.1^{\prime \prime}$ away. Since the optical HST spectrum is less affected by this contamination (de Koter et al. 1997), we primarily rely on this spectrum.

This contamination is probably the reason that our SED fit cannot simultaneously reproduce the continuum slope of the UV and optical HST spectrum. However, the optical spectrum can be matched in conformity with $K_{\mathrm{S}}$-band photometry (Crowther et al. 2010). Nevertheless, the luminosity of this star is subject to a large uncertainty, since the optical HST spectrum of BAT99 109 is also contaminated to a certain extent. Despite these uncertainties, we obtained nearly the same stellar parameters as previously derived by Crowther et al. (2010). With a luminosity of $\log \left(L / L_{\odot}\right)=6.69$, it is one of the most luminous objects in our sample. We point out that a fit of the photometry (Crowther \& Dessart 1998, BAT99; Crowther et al. 2010) alone does not result in a lower luminosity. Note that X-ray emission is associated with BAT99 109 (cf. comment on BAT99 106).

BAT99 110 was classified as O2 If* by Crowther \& Walborn (2011). The preceding studies by Heap et al. (1994) and de Koter et al. (1997) report nearly equal physical parameters. In contrast to these studies, our best fitting model has a stellar temperature of $T_{*}=50 \mathrm{kK}$ which is $7.5 \mathrm{kK}$ higher. Lower temperatures are excluded by our analysis, since, relative to the observed line strengths, our grid models with lower stellar temperatures overestimate the N III lines and underestimates the N IV and $\mathrm{N} v$ lines. This temperature discrepancy probably arises due to the inclusion of line blanketing in our models, which was not accounted for in the stellar atmosphere models at the time of the earlier studies. Moreover, our new study results in a luminosity which is factor of two higher, while the mass-loss rate is about $40 \%$ lower compared to the results obtained by Heap et al. (1994) and de Koter et al. (1997). We note that it is not possible to reproduce the UV and optical spectrum with the same reddening parameters. A satisfying fit of the UV spectra can only be achieved with a color excess of $E_{b-v}=0.1$ and a luminosity of $\log \left(L / L_{\odot}\right)=5.33$. However, these values are considerably low compared to the results (see Table 2) derived from the photometry (Crowther \& Dessart 1998; Breysacher et al. 1999) and the optical HST spectrum. Note that X-ray emission is associated with BAT99 110 (cf. comment on BAT99 106).

BAT99 111 is a WN9ha star (BAT99) in the center of R 136. Although Schnurr et al. (2009a) could not find radial velocity variations for this object, we treat it as a binary suspect because of the X-ray emission reported by Townsley et al. (2006). We have two flux-calibrated HST spectra at hand, but we note that the model cannot perfectly reproduce the continuum shape of the UV and the optical spectra with the same reddening parameters. However, the luminosity derived from the UV spectrum is only 0.06 dex lower than the value (Table 2) derived from the optical HST spectrum and optical photometry (BAT99). Due to a hydrogen mass-fraction of $X_{\mathrm{H}}=0.7$, this object appears not to be in an advanced evolution stage. Therefore, we disagree with the conclusion of Schnurr et al. (2009a) that this star is more evolved than the other stars in the core of R 136.

BAT99 112 is another WN5h star in the core of R 136 that is a candidate for a long-period binary system (Schnurr et al. 2009a). An indirect argument in favor of the binary status is the hard X-ray emission detected by Townsley et al. (2006) and Guerrero \& Chu (2008a). We expect that the potential companion does not contribute much to the bolometric luminosity, since no spectral lines of the potential companion can be recognized in the HST spectrum. Thus, we analyzed this star as a single star, despite its pending binary status.

In the SED fit, the slope of the calibrated HST spectrum and the optical photometry (BAT99) can be consistently reproduced with the same luminosity and color excess. In contrast, the $K_{r m S}$-band photometry (Crowther et al. 2010) exhibits a clear excess, which might be caused by the potential companion or dust emission. We note that the available HST spectrum does not cover the $\mathrm{H} \alpha$ and $\mathrm{H} \beta$ lines, so that the hydrogen abundance is derived using the higher members of the Balmer series alone. Since these lines are rather weak, the hydrogen abundance is subject to a relatively high uncertainty. We estimate a hydrogen massfraction of $X_{\mathrm{H}}=0.2$, which is 0.1 dex lower than previously derived by Crowther et al. (2010). The temperature obtained by these authors is slightly lower, while the mass-loss rate and the luminosity is more than 0.2 dex higher compared to the results of the present paper.

BAT99 113 is a transition type O2 If*/WN5 star (Crowther \& Walborn 2011; Evans et al. 2011) located close to the core of 30 Doradus. We have two optical spectra at hand, an archival HST spectrum and a ground-based spectrum taken by Schnurr et al. (2008). These spectra, however, clearly deviate from each other. For example, the equivalent width of the He II $\lambda 4686$ line differ by roughly a factor of two. Although the $\mathrm{S} / \mathrm{N}$ is lower in the HST spectrum, we primarily use this spectrum in our analysis because of the distinctive spatial resolution of the HST. The 
star was identified by Schnurr et al. (2008) as a binary system with a period of $4.7 \mathrm{~d}$. Since no indications of a companion were found in the spectra, we expect that the flux contribution of the companion is insignificant for the analysis of the WN star. Unfortunately, the HST spectra do not cover the $\mathrm{H} \alpha$ and $\mathrm{H} \beta$ lines. Consequently, the hydrogen abundance given in Table 2 is derived from weak $\mathrm{H} \gamma$ and $\mathrm{H} \delta$ lines alone.

BAT99 114 is another transition type O2 If*/WN5 star (Crowther \& Walborn 2011; Evans et al. 2011) in the vicinity of the 30 Doradus core. We have two optical spectra at hand, an archival HST spectrum and a spectrum obtained by Schnurr et al. (2008). Since the latter lacks a sufficient subtraction of the diffuse background, our analysis is mainly based on the HST spectrum. However, this spectrum covers only the wavelength range from 3300 to $4800 \AA$, where merely the $\mathrm{H} \gamma$ and $\mathrm{H} \delta$ lines can be found as indicators for the hydrogen abundance. Due to the X-ray emission detected by Guerrero \& Chu (2008a) we consider this object as a binary suspect, although Schnurr et al. (2008) could not find periodic radial velocity variations.

BAT99 116 was classified as WN5h:a by Schnurr et al. (2008). These authors have reported radial velocity variations, but found no periodicity. Schnurr et al. (2009a) noted that this object is likely a long periodic binary system, in agreement with the strong X-ray emission detected by Guerrero \& Chu (2008a,b). Thus, we consider this object as a binary suspect. Unfortunately, the only spectrum at hand (Schnurr et al. 2008) lacks a subtraction of the diffuse background. Therefore, we are not able to establish a robust estimate of the hydrogen abundance.

BAT99 117 is a WN5ha star (Foellmi et al. 2003b) located in the northern part of 30 Doradus. The stellar temperature and luminosity derived in our analysis are moderately lower compared to the results obtained by HK2000, whereas the mass-loss rate is $50 \%$ higher. We also derive a 0.1 dex higher hydrogen abundance. However, our analysis suffers from an insufficient subtraction of the diffuse background in the spectrum from Foellmi et al. (2003b). This spectrum exhibits strong absorption lines in place of the O III-nebular emission lines at $4959 \AA$ and $5007 \AA$, which might be caused by an overcorrection of the background. If this is true, the Balmer series will probably be impaired by the inadequate nebular subtraction as well. In this case, the hydrogen abundance listed in Table 2 is a subject to high uncertainty.

By comparing the HRD position to stellar evolution models performed by Meynet \& Maeder (2005), we derive an initial mass of roughly $M_{\text {init }}=120 M_{\odot}$. Thus, this star belongs to the category of very massive stars. Foellmi et al. (2003b) did not find periodic radial velocity variations.

BAT99 118 is a WN6h star (BAT99), which is treated as a binary candidate in the radial velocity study by Schnurr et al. (2008). An indirect argument in favor of the binary status is the strong and hard X-ray flux detected by Guerrero \& Chu (2008a). New X-shooter observations performed by Sana et al. (2013b) revealed it to be a SB2 binary with a mass ratio close to unity. Thus, the system consists of two similar WN stars, which were classified as WN5-6h + WN6-7h by Sana et al. (2013b). We analyzed this system as if it were a single star.

Our best fit is achieved at a stellar temperature of $T_{*}=$ $47 \mathrm{kK}$, which is only marginally higher than the value recently derived by Doran et al. (2013). The mass-loss rate obtained by these authors agrees well with the value presented in this work. In contrast, Crowther \& Dessart (1998) obtained a mass-loss rate that is a factor of two lower, while the stellar temperature derived by these authors (on the basis of unblanketed model atmospheres) is $10 \mathrm{kK}$ lower. Crowther \& Dessart (1998) and Doran et al. (2013) obtain luminosities that are lower compared to the value $\left(\log \left(L / L_{\odot}\right)=6.66\right)$ derived in this work. Crowther \& Dessart (1998) obtained a value that is 0.32 dex lower, whereas the analysis carried out by Doran et al. (2013) results in a luminosity that is 0.25 dex lower. As opposed to this, the luminosity estimate by Sana et al. (2013b) results in a luminosity that is 0.14 dex higher compared to our new results.

These differences need to be considered, if the luminosity is used to derive the current mass of the stellar content. On the basis of their high luminosity, Sana et al. (2013b) derived a current mass between $80 M_{\odot}$ and $205 M_{\odot}$ for each component in BAT99 118. For the initial masses, we obtained about $M_{\text {init }}=100 M_{\odot}$ for each WN component by comparing the empirical HRD position of this object (see Figs. 7 and 10) with the stellar evolution tracks by Meynet \& Maeder (2005) and Yusof et al. (2013).

A description of the FUSE spectra (not considered in this work) can be found in Willis et al. (2004). The authors derived a terminal velocity of $v_{\infty}=1847 \mathrm{~km} \mathrm{~s}^{-1}$ which is about $250 \mathrm{~km} \mathrm{~s}^{-1}$ higher than the value used for the calculation of our grid models.

BAT99 119 is a WN6h star, which is listed as a single-line spectroscopic binary (SB1) in the BAT99 catalog with a period of $25.2 \mathrm{~d}$ (Moffat 1989). In contrast, Schnurr et al. (2008, $2009 \mathrm{~b}$ ) find a period of $158.8 \mathrm{~d}$, combining their radial velocity data with that of Moffat (1989) and new polarimetric data. According to Schnurr et al. (2009b), the companion is most likely an O-type star, although no obvious trace of the companion can be found in the spectrum. With the exception of a slightly smaller emission-line strength, the spectrum of BAT99 119 resembles that of BAT99 118, which is a SB2 of two similar WN stars (Sana et al. 2013b). Considering that the resolving power of our optical spectra is only $R \approx 1000$, we also stress the possibility of a binary system encompassing two WN stars with a mass ratio close to unity.

Assuming an O-type companion, Schnurr et al. (2009b) were able to give constraints on the properties of both components in this binary system. They found the WN star to be the considerably more luminous component. Thus, we estimate the flux contribution of the companion to be negligible in the UV and optical spectral range, which is in accordance with the moderate infrared excess found in our analysis.

Similar to BAT99118, we derive a stellar temperature of $T_{*}=47 \mathrm{kK}$, which is about $15 \mathrm{kK}$ higher than that obtained by Crowther \& Smith (1997) on the basis of unblanketed model atmospheres. In comparison to this former study, our substantially higher temperature entails a luminosity increase by a factor of roughly $2.5\left(\log \left(L / L_{\odot}\right)=6.57\right)$. The mass-loss rate derived here, on the other hand, is nearly identical to the value given by Crowther \& Smith (1997), whereas Schnurr et al. (2009b) estimated a mass-loss rate on the basis of their polarimetric data, which is a factor of two higher.

The latter authors derived a dynamical mass of $M_{\text {dyn }}=116 \pm$ $33 M_{\odot}$ for the WN component. The initial mass obtained from the HRD position (Figs. 7 and 10) will be approximately $M_{\text {init }}=$ $150 M_{\odot}$, if the WN star contributes most to the overall flux of the binary system $(\mathrm{WN}+\mathrm{OB})$. 
BAT99 120 is classified as WN9h star (BAT99) and may be a dormant LBV, according to Crowther et al. (1995a). We have two optical spectra at hand, an archival AAT spectrum (see Sect. 2.3) and a coadded spectrum observed by Foellmi et al. (2003b). The stellar parameters presented in Table 2 rely mainly on the latter spectrum, due to their high $\mathrm{S} / \mathrm{N}$. This spectrum is best reproduced by a model with a stellar temperature of $T_{*}=32 \mathrm{kK}$, while a model with $T_{*}=35 \mathrm{kK}$ is more appropriate for the AAT spectrum. BAT99 120 was previously analyzed by Pasquali et al. (1997) and Crowther et al. (1995a). They obtained stellar temperatures of $T_{*}=38.9 \mathrm{kK}$ and $T_{*}=30 \mathrm{kK}$, respectively. However, stellar temperatures higher than $T_{*}=35 \mathrm{kK}$ would spoil the fit of the He I and He II lines in both optical spectra.

In addition to the optical spectra, we used flux-calibrated UV spectra, which were obtained with the HST and the IUE satellite. Fitting the continua of these spectra and the available photometric data (2MASS and optical photometry from the BAT99 catalog), the luminosity is found to be $\log \left(L / L_{\odot}\right)=$ 5.58 , while the color excess amounts to $E_{b-v}=0.15$. In contrast, the luminosities presented by Crowther et al. (1995a) and Pasquali et al. (1997) are higher, while the derived color excess agrees with our study. The luminosity derived by Crowther et al. (1995a) is only slightly higher, whereas Pasquali et al. (1997) derived a luminosity of a factor of 2.5 higher. This deviation can be attributed the higher temperature and thus higher bolometric correction derived by Pasquali et al. (1997). In comparison to the former studies, the mass-loss rate is slightly lower in our new study.

BAT99 122 had never been analyzed by means of model atmospheres before. The infrared excess reported by Hyland et al. (1978) can be seen in our fit of the SED (Fig. C.49) as well. The star was classified as WN5h by Evans et al. (2011).

BAT99 124 belongs to the WN4 subclass (Foellmi et al. 2003b). It is analyzed by means of model atmospheres for the first time in this work. The uncertainty of the obtained hydrogen abundance is large, since the available spectrum is strongly contaminated with nebular emission, which is evident by the strong O III $\lambda \lambda 4959,5007$ nebular emission lines. According to Martín-Hernández et al. (2005), the shell structure of NGC 2077 may be caused by the feedback of BAT99 124 .

BAT99 126 is listed in the BAT99 catalog as a WN3+O7 binary candidate. Foellmi et al. (2003b) found a period of $25.5 \mathrm{~d}$, but noted that more data are needed to verify this result. Therefore, we treat this object as a binary suspect, although it is likely a binary. The X-ray emission detected by Guerrero \& Chu (2008a) is further indirect evidence for the binary status. Foellmi et al. (2003b) reclassified the companion to $\mathrm{O} 8$ and the WN component to WN4b. The luminosity of this object is derived from photometry only, since no flux-calibrated spectra are available.

BAT99 128, classified as WN3b (Foellmi et al. 2003b), is a typical WN3 star. An observational discrepancy exists for this star between the photometry obtained by Crowther \& Hadfield (2006) and the flux-calibrated spectrum obtained by Torres-Dodgen \& Massey (1988). The noisy spectrum measured by Torres-Dodgen \& Massey (1988) exhibits a higher flux, which results in a SED fit of only moderate quality and an unreliably low color excess of $E_{b-v}=0.01 \mathrm{mag}$. The spectrophotometry from Crowther \& Hadfield (2006) fits much bet- ter to the 2MASS and the IRAC photometry and results in a SED fit of higher quality and a more convincing color excess of $E_{b-v}=0.17$ mag. Foellmi et al. (2003b) found a radial velocity that is significantly below the mean $v_{\text {rad }}$ of their sample, suggesting that this star might be a runaway. We note that BAT99 128 falls into the regime of parameter degeneracy (cf. Sect. 4.2).

BAT99 129 is an eclipsing binary with a WN3(h)a star (BAT99) as primary component and an O5V companion (Foellmi et al. 2006). Foellmi et al. (2003b) find a radial velocity period of $2.76 \mathrm{~d}$, but no X-ray emission was detected by the Rosat satellite (Guerrero \& Chu 2008b). We estimate a hydrogen mass-fraction of $X_{\mathrm{H}}=0.2$, thus confirming the above classification. Foellmi et al. (2006) derived a luminosity of $\log \left(L / L_{\odot}\right)=4.97$ for the WR component, which would make this the faintest WN star known in the LMC (cf. Fig. 7). This result cannot be confirmed by our analysis. Assuming that the luminosity derived in our analysis is valid for the whole system, and applying the luminosity ratio of 0.3 derived by Foellmi et al. (2006), we obtain a luminosity of $\log \left(L / L_{\odot}\right)=5.68$ for the WN component. This luminosity is considerably higher than the value derived by Foellmi et al. (2006) and at the upper end of the luminosity range derived for the other presumably single WN3 stars in the LMC.

BAT99 130 is the second WN11h star (BAT99) in our sample. Our new analysis confirms the stellar parameters derived in the former study by Crowther \& Smith (1997) with the exception of a significantly lower hydrogen abundance.

BAT99 131 was classified as WN4b (Foellmi et al. 2003b) and had not been analyzed before. The available IUE spectra are not uniform and do not exhibit any prominent emission line. Therefore, we reject these spectra from our analysis, although the flux of the IUE long-wavelength spectrum is compatible to the available photometry. Thus, the luminosity obtained in our analysis is derived from visual narrowband, 2MASS and IRAC photometry alone.

BAT99 132 is a WN4b(h) star (Foellmi et al. 2003b), analyzed for the first time in this paper. The best fit of the spectra is achieved with a hydrogen-free model, although the presence of residual hydrogen in the stellar atmosphere of this object was reported by Foellmi et al. (2003b).

BAT99 133 is the third WN11h (BAT99) in the LMC and one of the only three WN stars detected at $24 \mu \mathrm{m}$ with the IRAC instrument aboard the Spitzer space telescope (Bonanos et al. 2009). According to Humphreys \& Davidson (1994) and Weis (2003), this star is suspected to be an LBV in its quiescent phase. Likewise, Walborn (1982) and Bonanos et al. (2009) have noted the spectroscopic similarities between BAT99 133 and the LBV BAT99 83 in its minimum. Contrary to this, our spectra of these two stars exhibit clear differences. For example, the He II $\lambda 4686$ line is absent in the spectrum of BAT99 83, whereas a relatively small emission line is present in the spectrum of BAT99 133. Further different features are the He I lines, which are much more prominent in the spectrum of BAT99 133.

BAT99 133 was previously analyzed by Crowther \& Smith (1997) and Pasquali et al. (1997). The former obtained a stellar temperature of $T_{*}=28.3 \mathrm{kK}$, which is confirmed by our analysis. Pasquali et al. (1997), on the other hand, derived a stellar temperature of roughly $8 \mathrm{kK}$ higher. However, our models 
clearly underpredict the observed $\mathrm{He}$ I/He II ratio at this higher temperature. In principle, the same applies to the mass-loss rate and luminosity, where we can confirm the results obtained by Crowther \& Smith (1997). Contrary to this, Pasquali et al. (1997) derived values for the mass-loss rate and the luminosity that are roughly twice as high.

A study of the nebula associated with BAT99 133 can be found in Pasquali et al. (1999) and Weis (2003).

BAT99 134 is listed as WN4b star in the BAT99 catalog. In this first spectroscopic analysis with stellar atmosphere models, we derived physical parameters typical for the WN4 subclass.

Dopita et al. (1994) discovered a ring nebula that surrounds BAT99 134. As no He II nebular emission is detected by (Nazé et al. 2003b), they obtain an upper limit for the number of He II ionizing photons delivered by the exciting star, which amounts to $<3.2 \times 10^{45} \mathrm{He}$ II ionizing photons per second. This agrees with our final model, which does not produce a significant number of He II ionizing photons (see Table A.3).

\section{Appendix C: Spectral fits}

In this section, we present the spectral fits of all stars analyzed in this study. The individual plots encompass the fit of the spectral energy distribution (top panel) to the photometric and fluxcalibrated spectra as well as the fits to the normalized optical and UV spectra (lower panels), when available. The observations are plotted in blue, whereas the synthetic spectrum of the best-fitting model shown in red.

Some of our stellar atmosphere models with stellar temperatures below $T_{*}=32 \mathrm{kK}$ exhibit spurious emission lines in the spectral range from about $1900 \AA$ to $2100 \AA$. These emission features, which are not observed, originates from the third ionization stage of our generic model atom representing the iron-group elements. We note that the presence of these emission features is only a cosmetic issue and has no impact on the derived stellar parameters. 

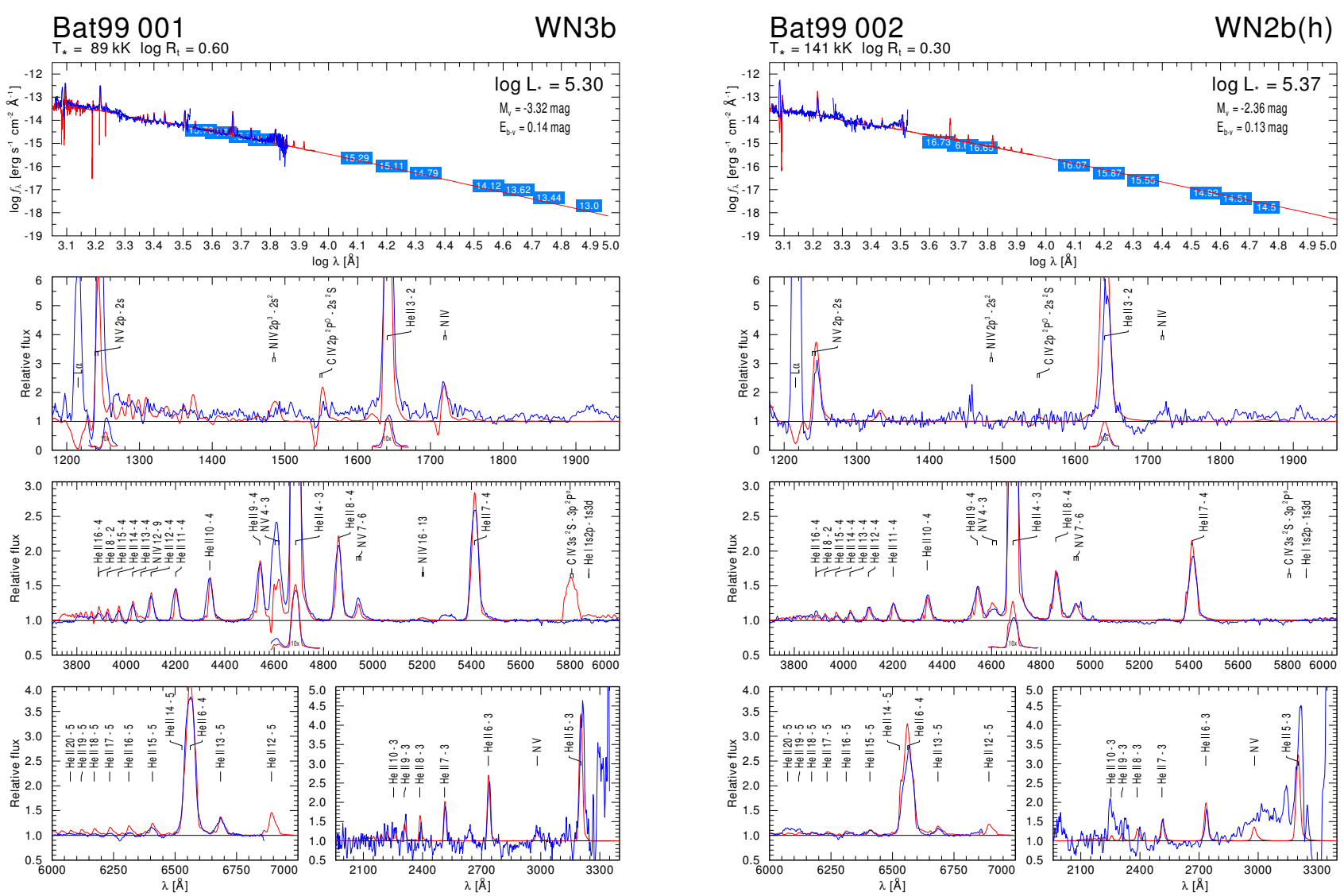

Fig. C.1. Spectral fit for BAT99 001 and BAT99 002.
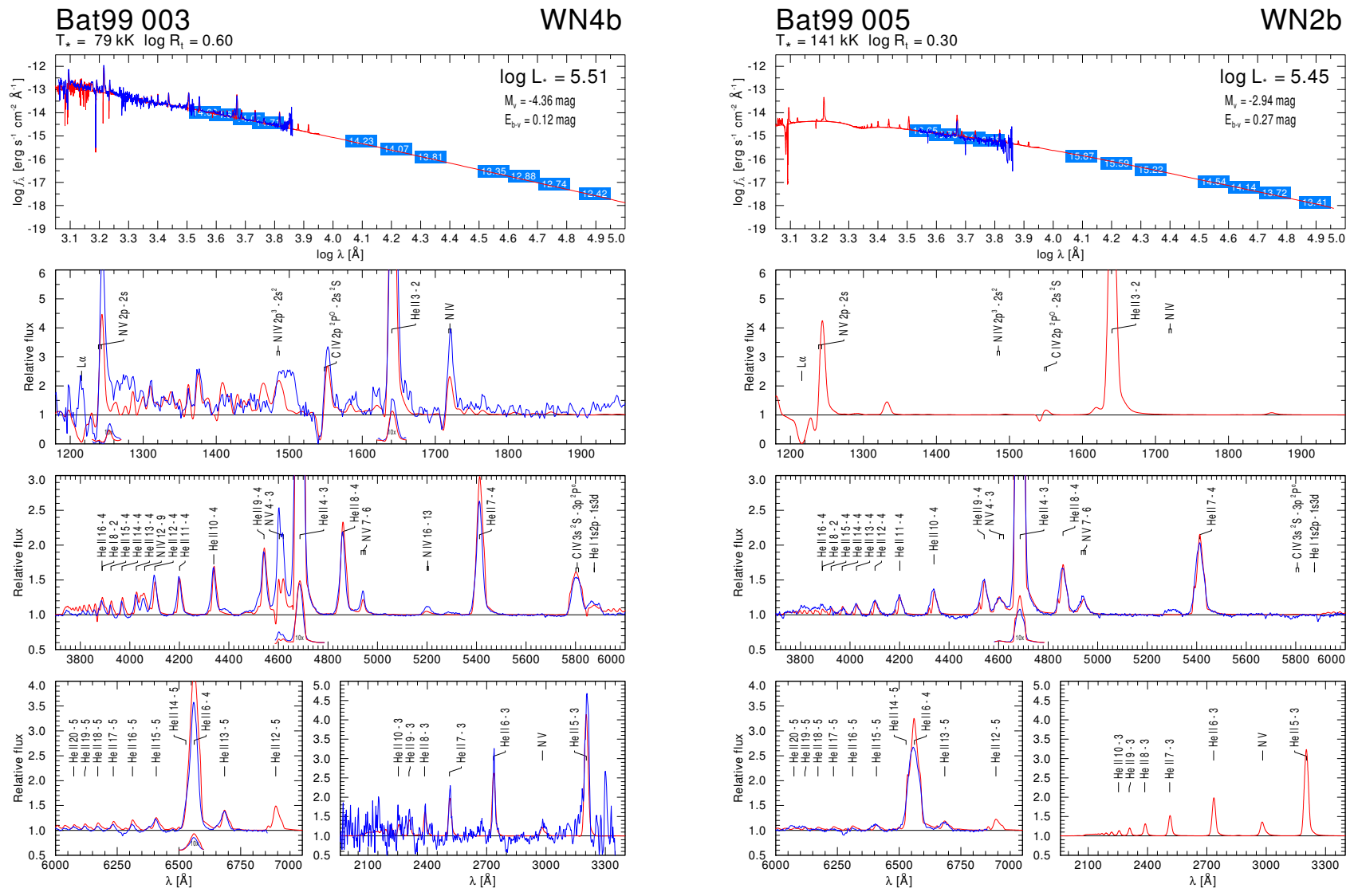

Fig. C.2. Spectral fit for BAT99 003 and BAT99 005. 
R. Hainich et al.: The Wolf-Rayet stars in the Large Magellanic Cloud

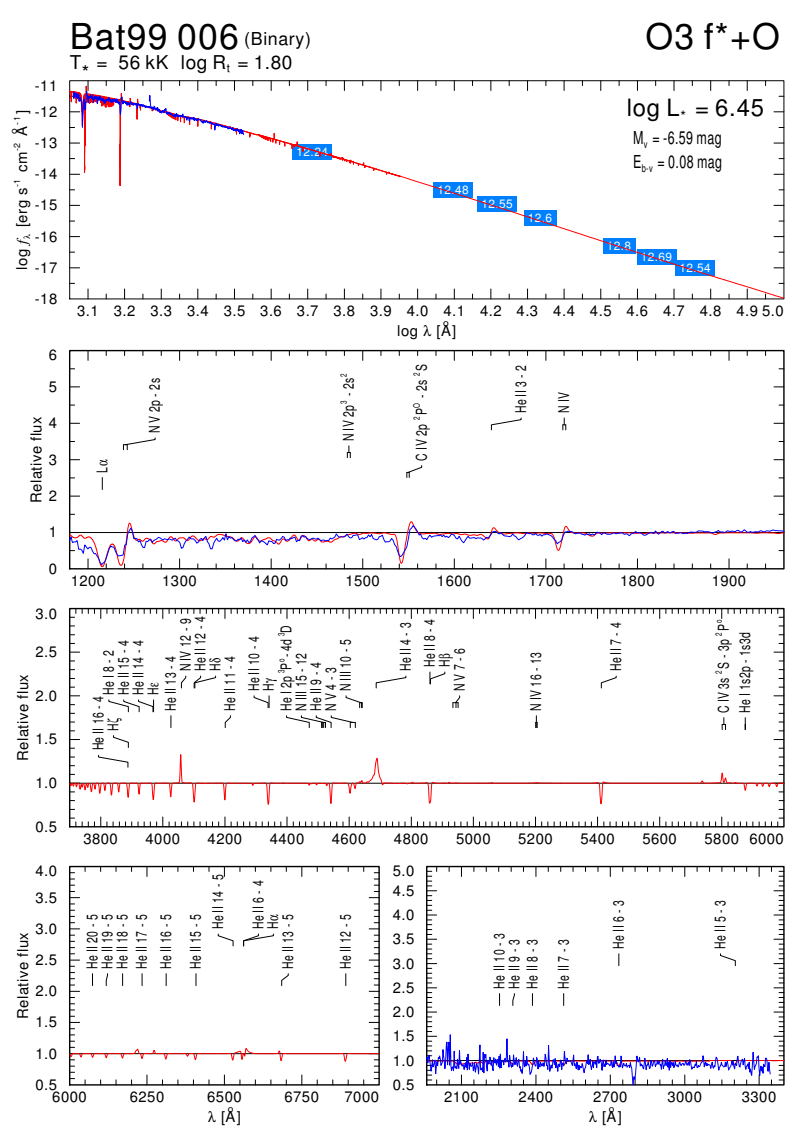

Bat99 007

WN4b
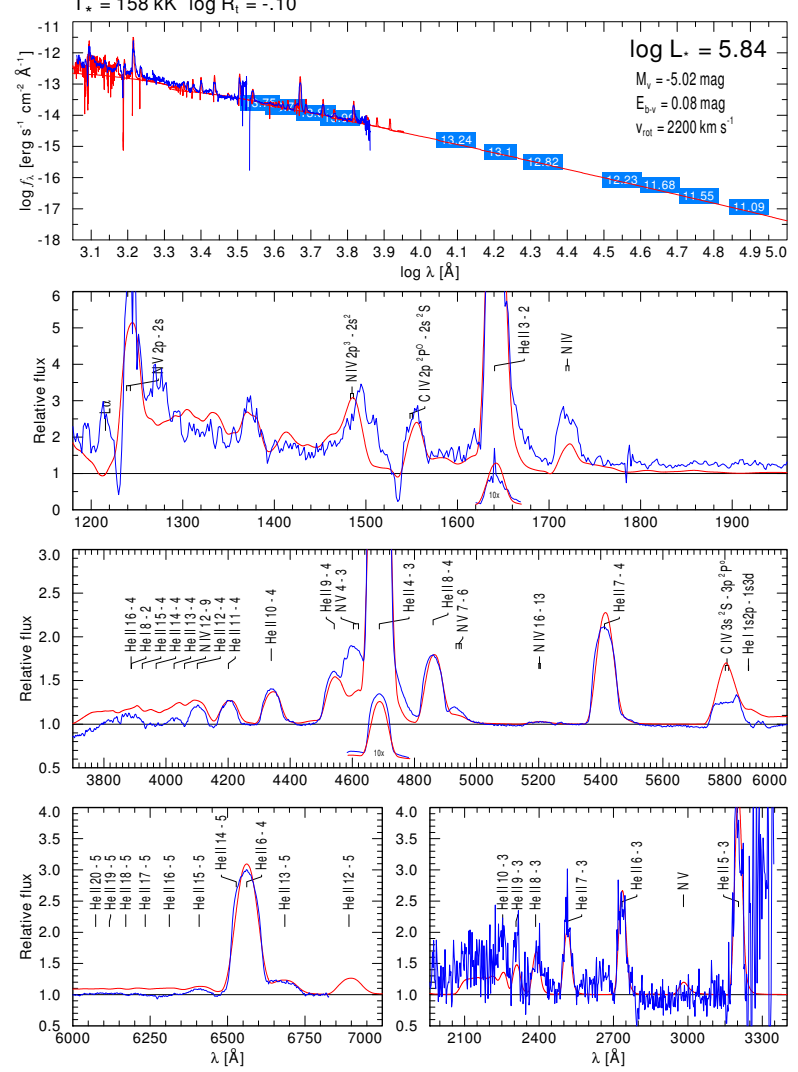

Fig. C.3. Spectral fit for BAT99 006 and BAT99 007.
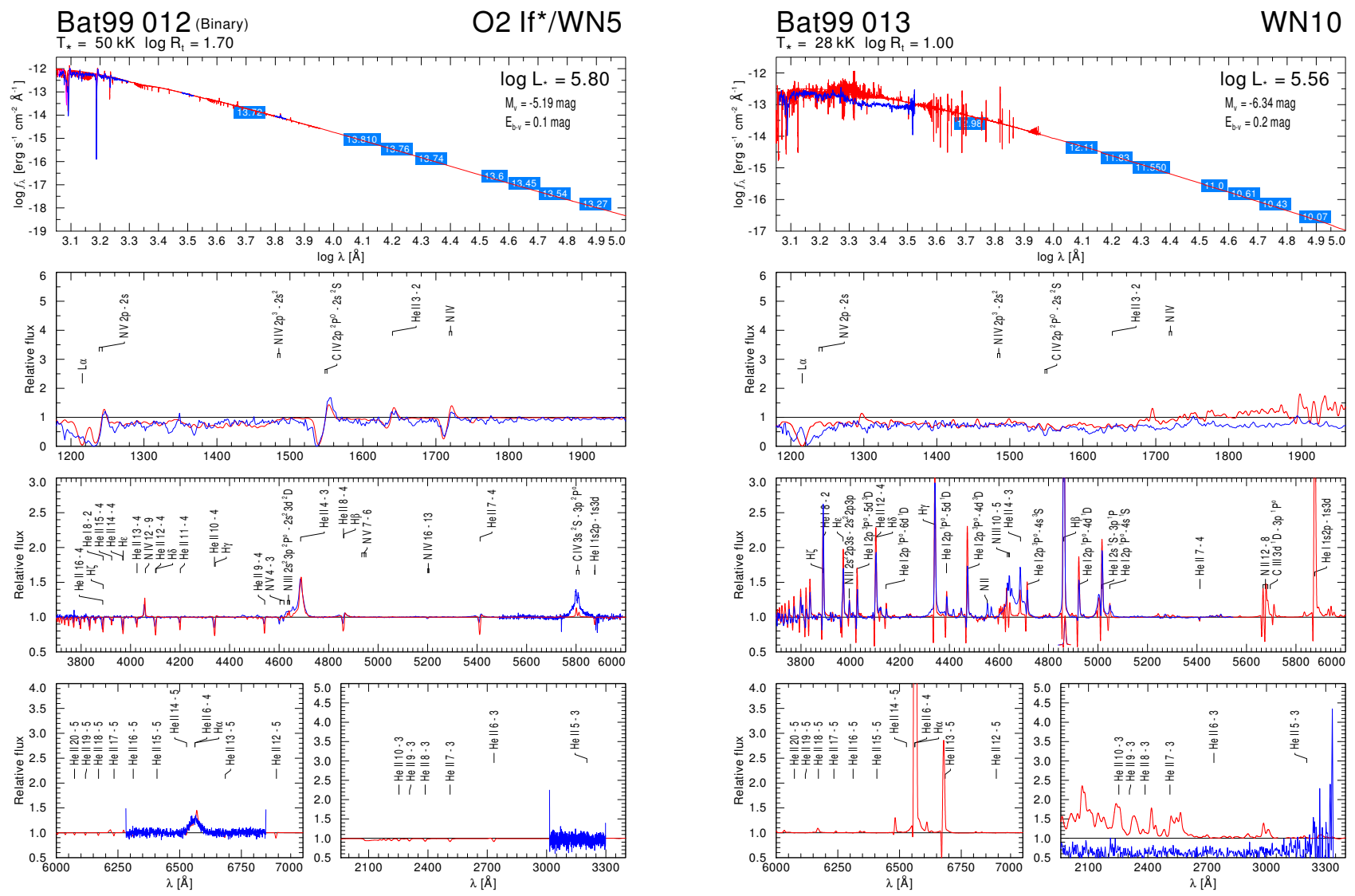

Fig. C.4. Spectral fit for BAT99 012 and BAT99 013. 


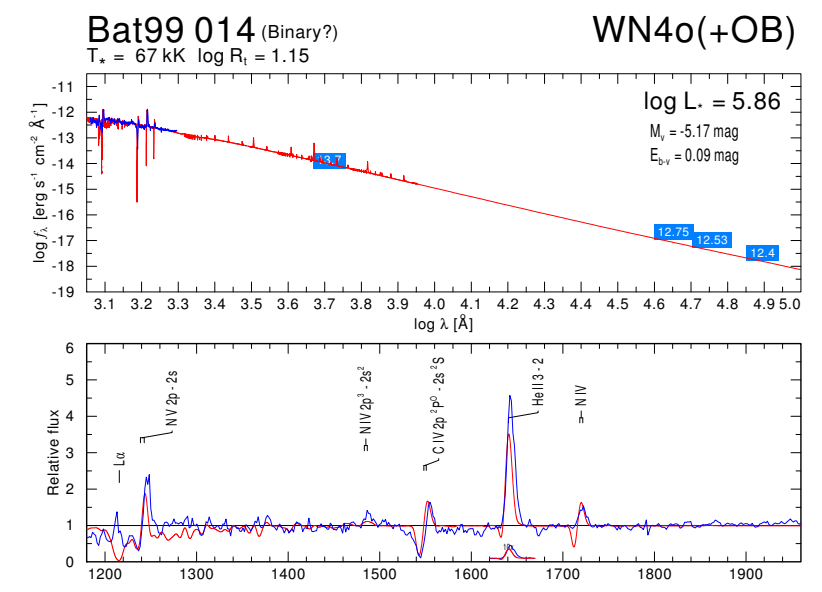

Bat99 015

WN4b
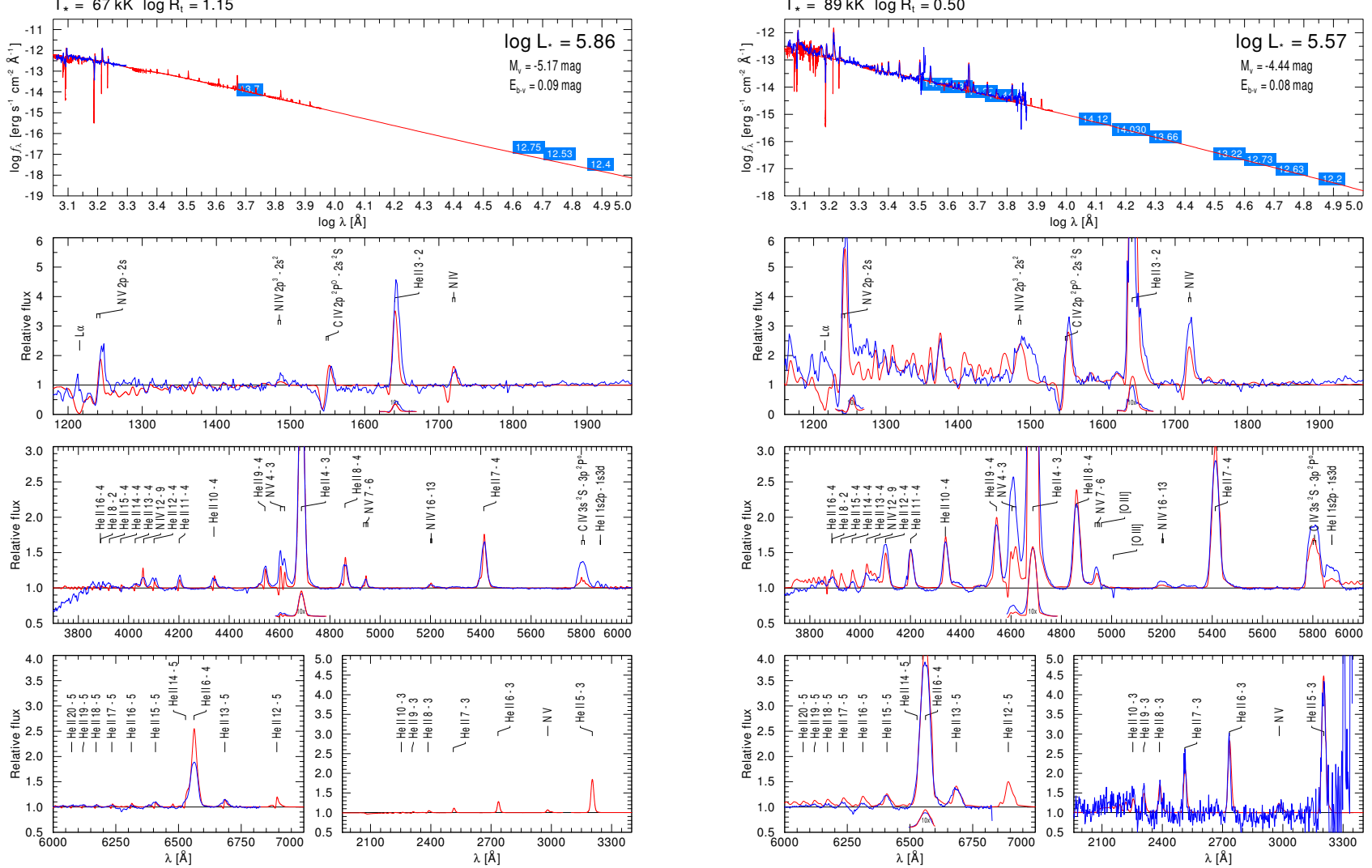

Fig. C.5. Spectral fit for BAT99 014 and BAT99 015.
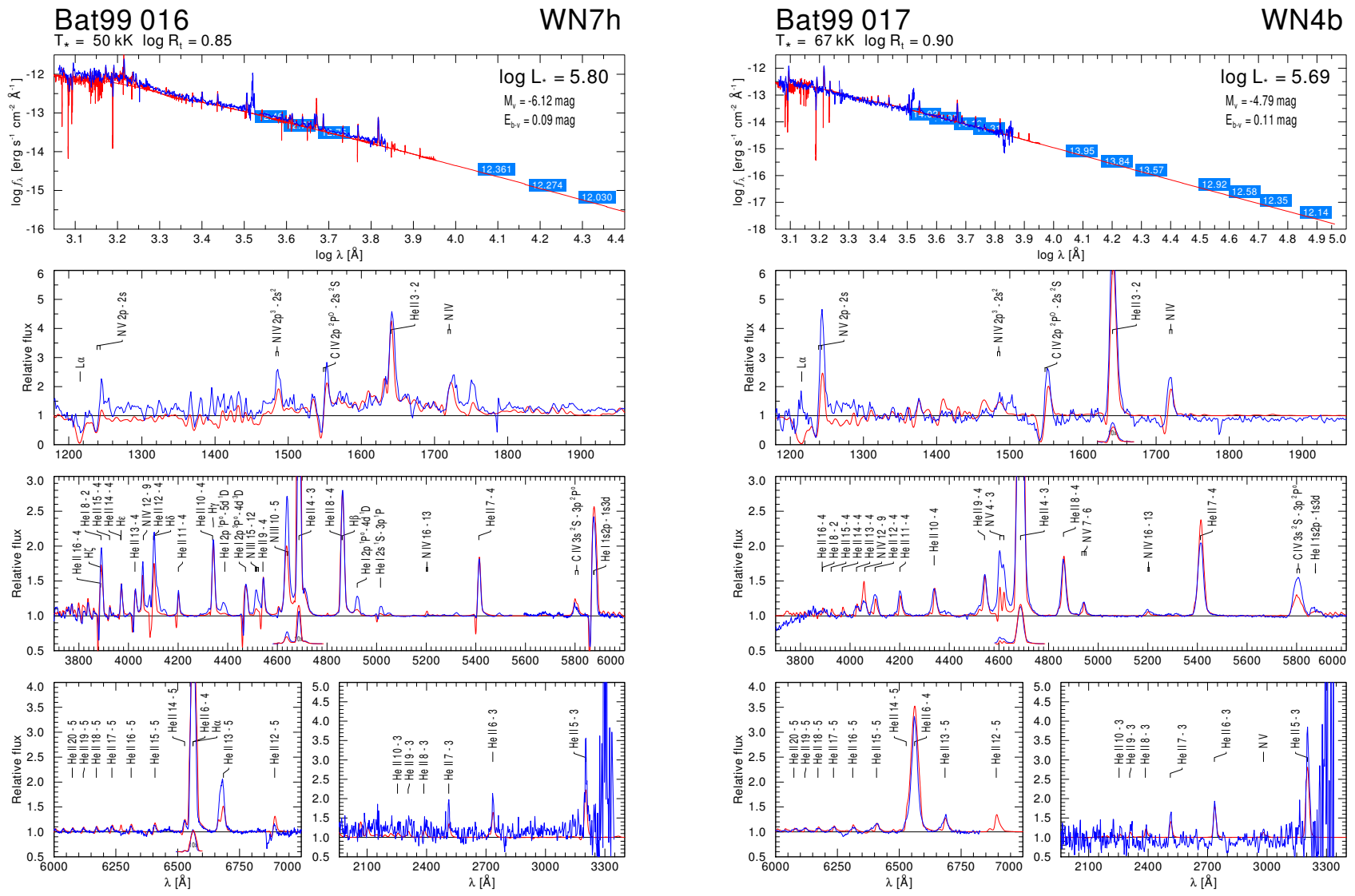

Fig. C.6. Spectral fit for BAT99 016 and BAT99 017. 
R. Hainich et al.: The Wolf-Rayet stars in the Large Magellanic Cloud
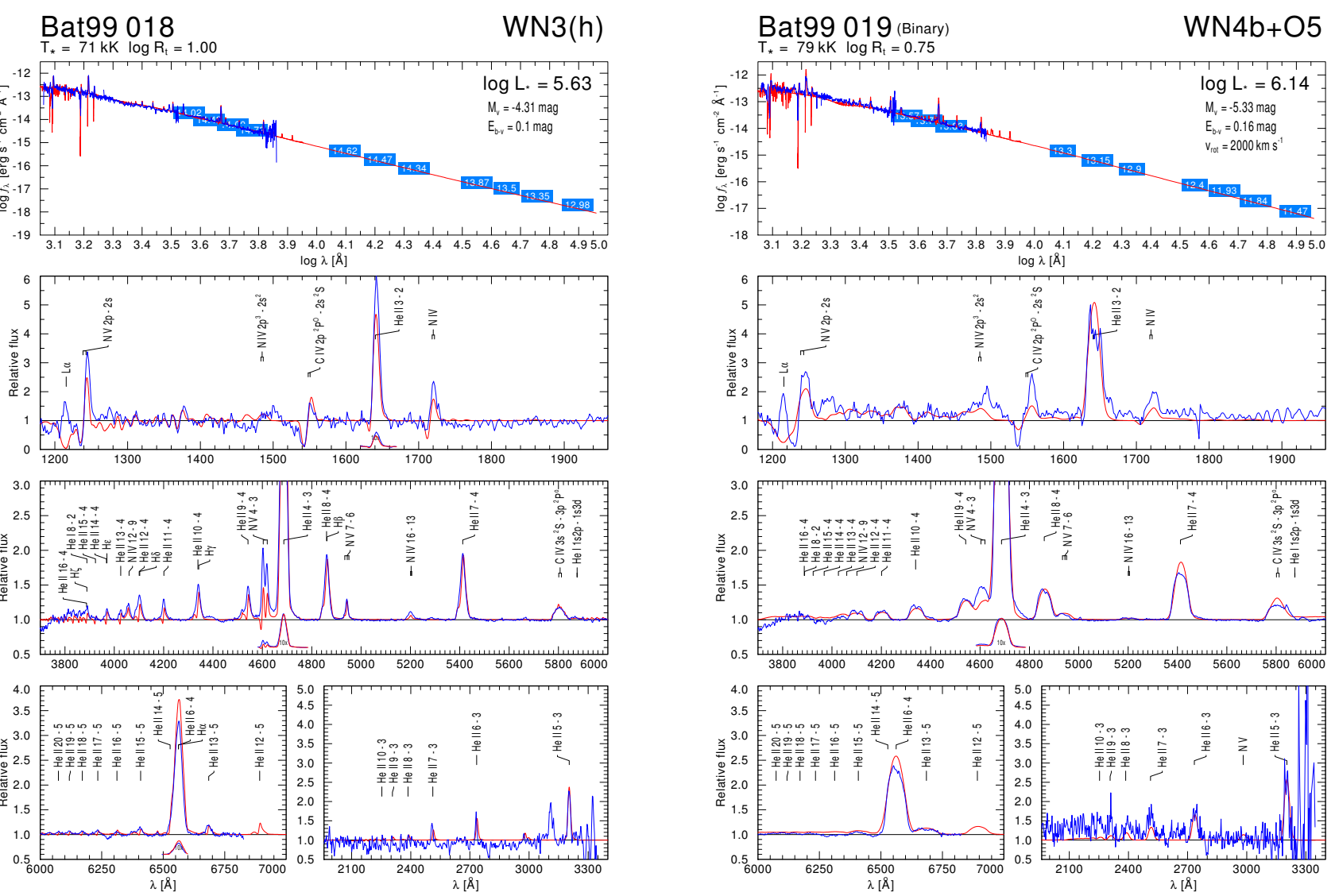

Fig. C.7. Spectral fit for BAT99 018 and BAT99 019.
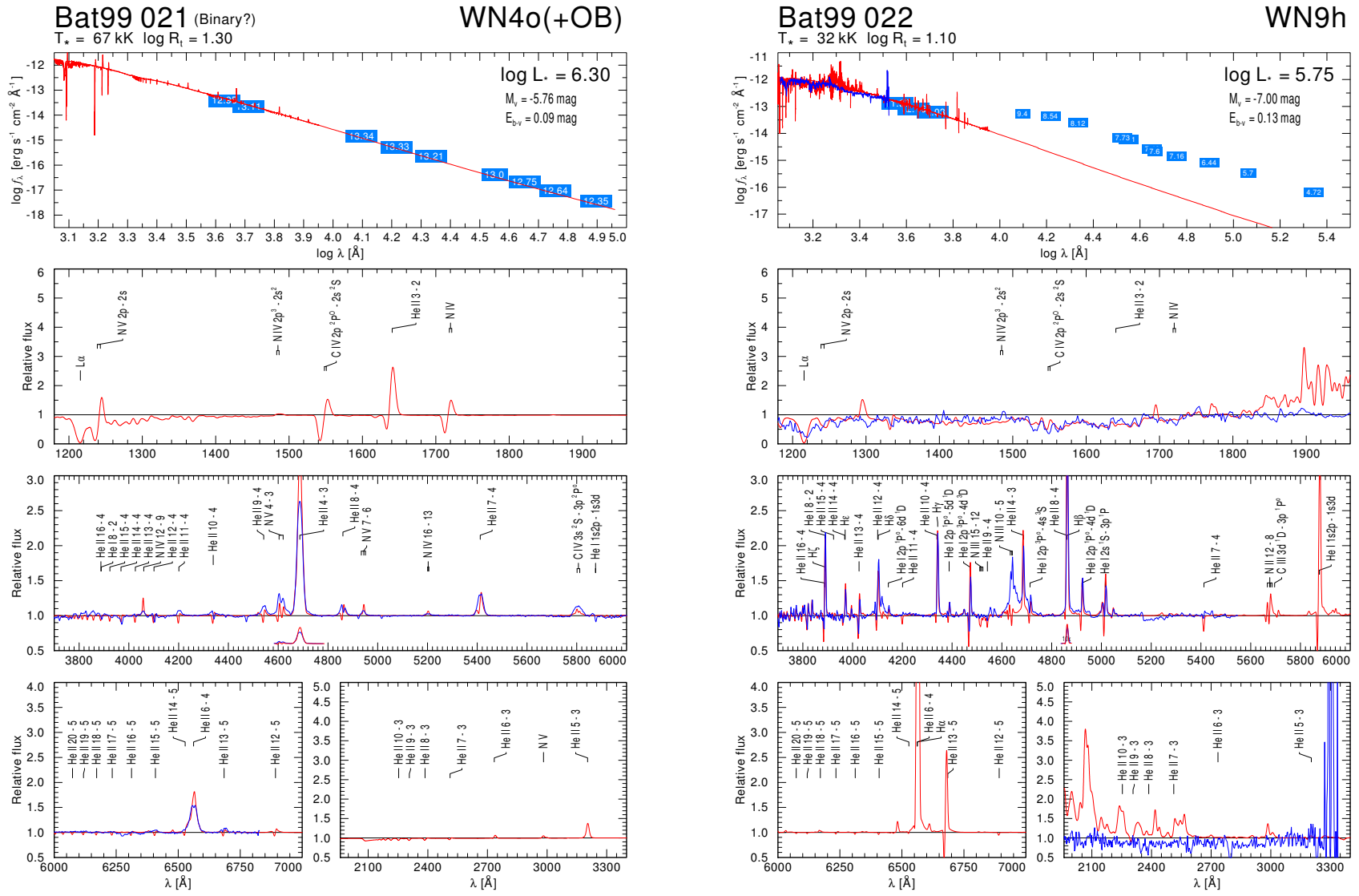

Fig. C.8. Spectral fit for BAT99 021 and BAT99 022. 
Bat99 023
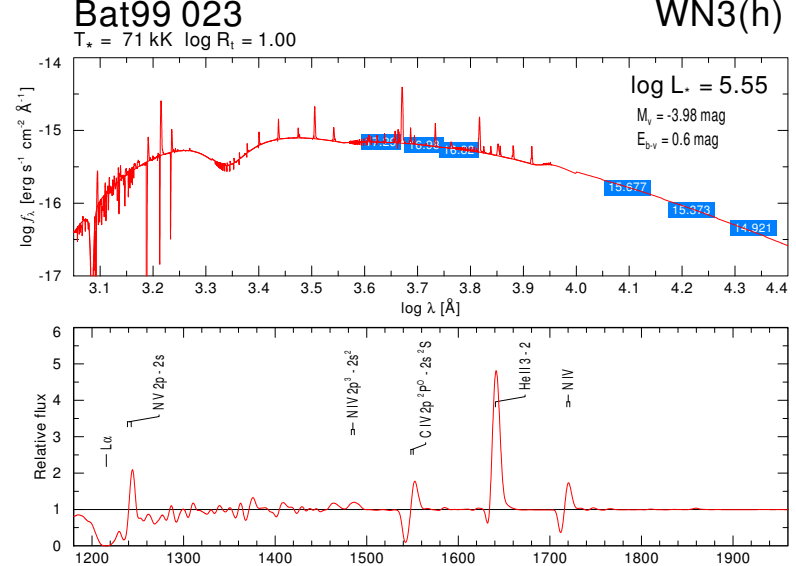

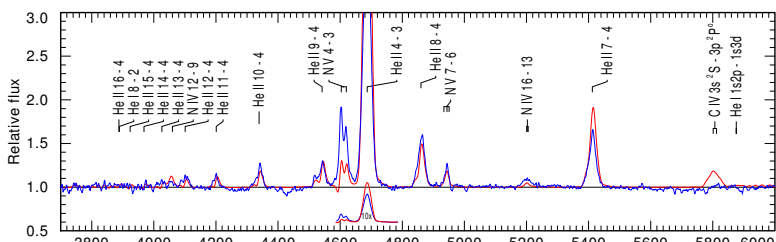

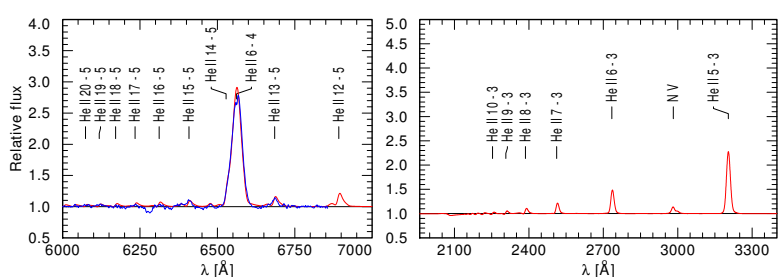

Fig. C.9. Spectral fit for BAT99 023 and BAT99 024.
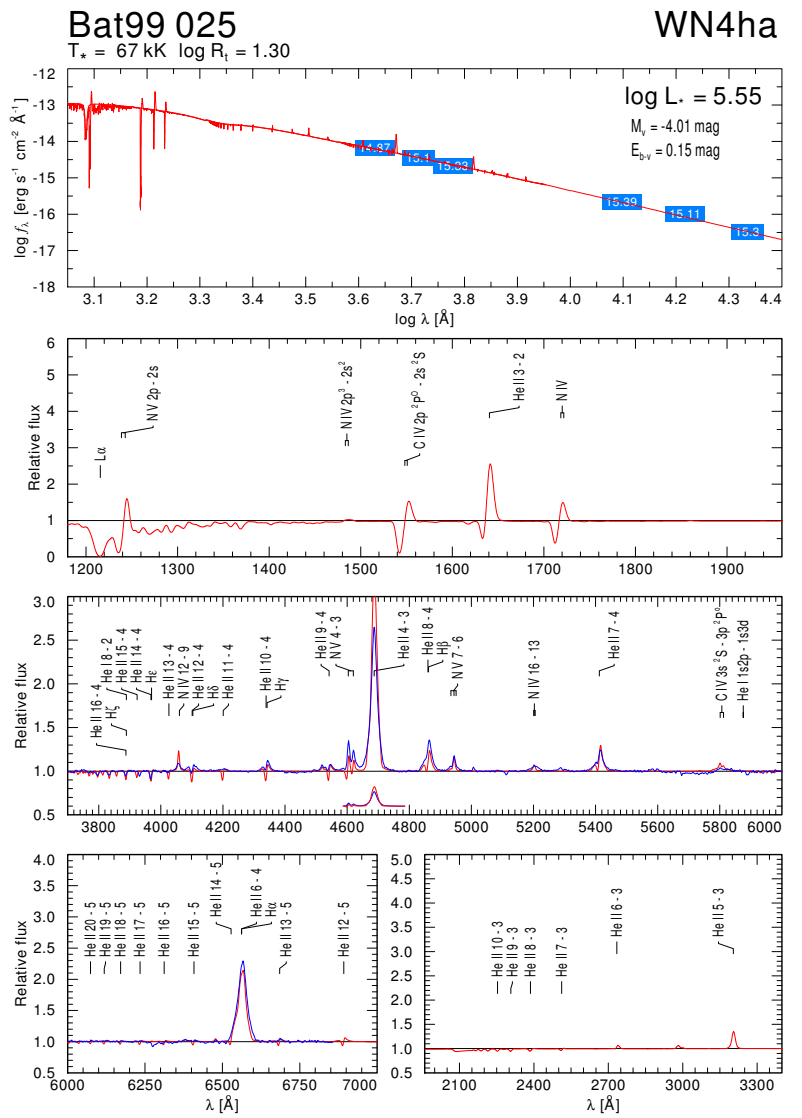

Fig. C.10. Spectral fit for BAT99 025 and BAT99 026.
Bat99 024

WN4b
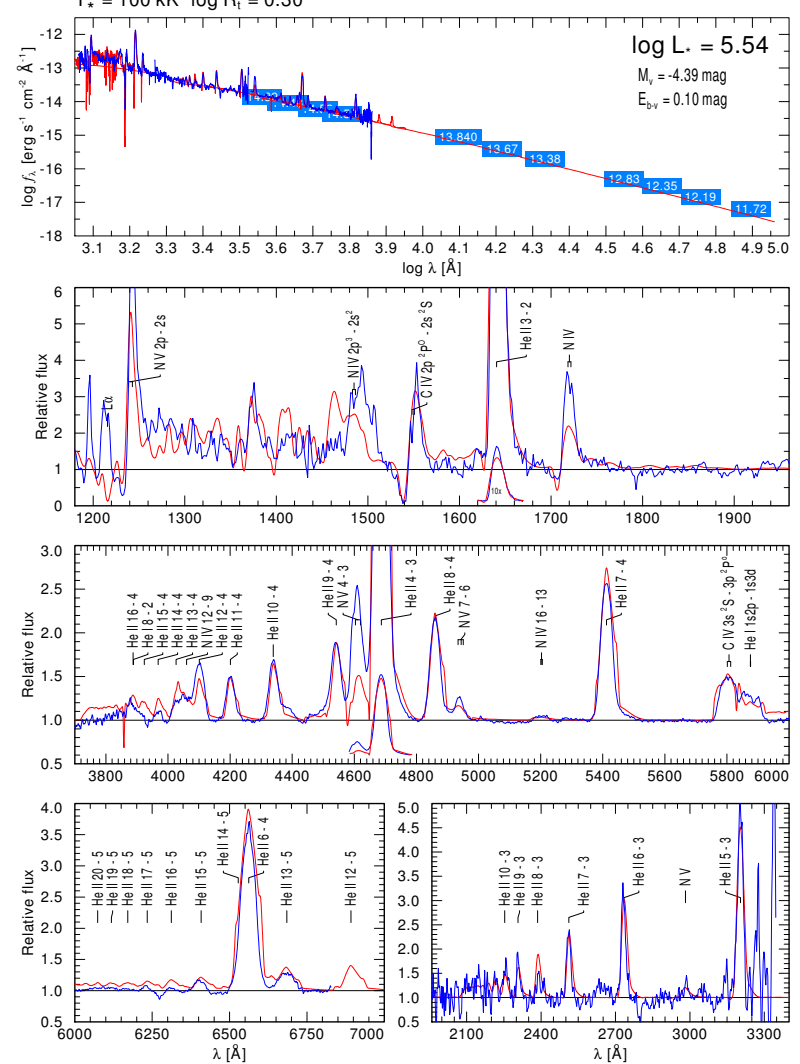

Bat99 026

WN4b
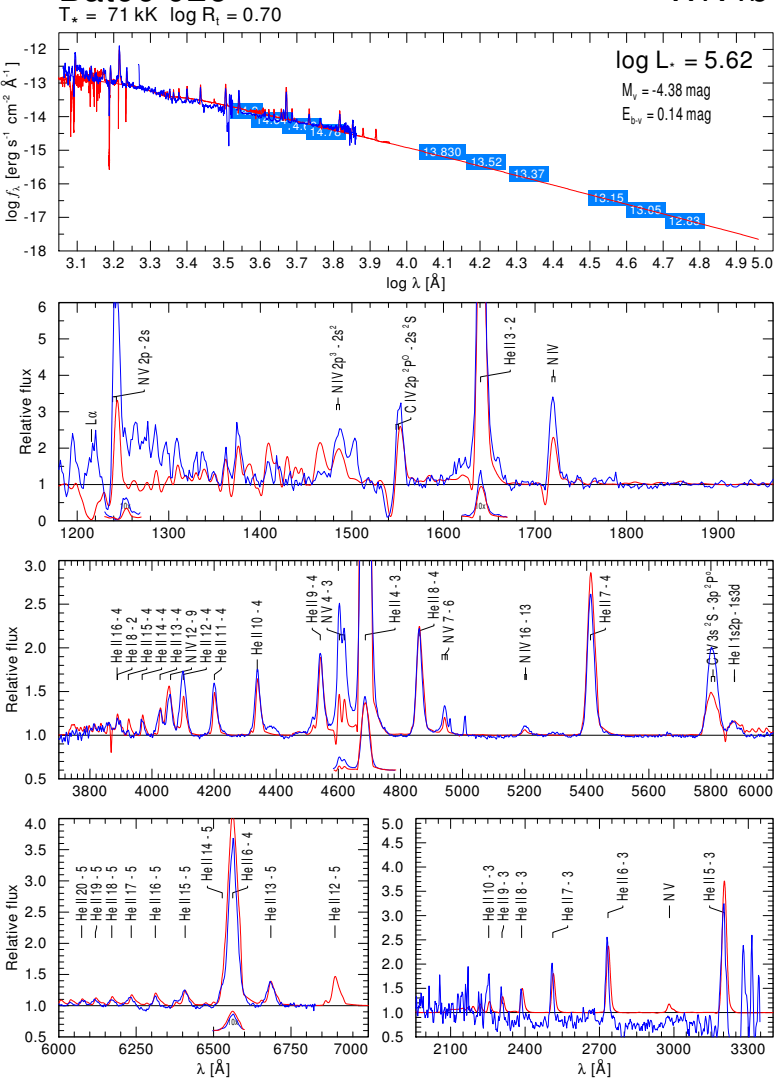
R. Hainich et al.: The Wolf-Rayet stars in the Large Magellanic Cloud
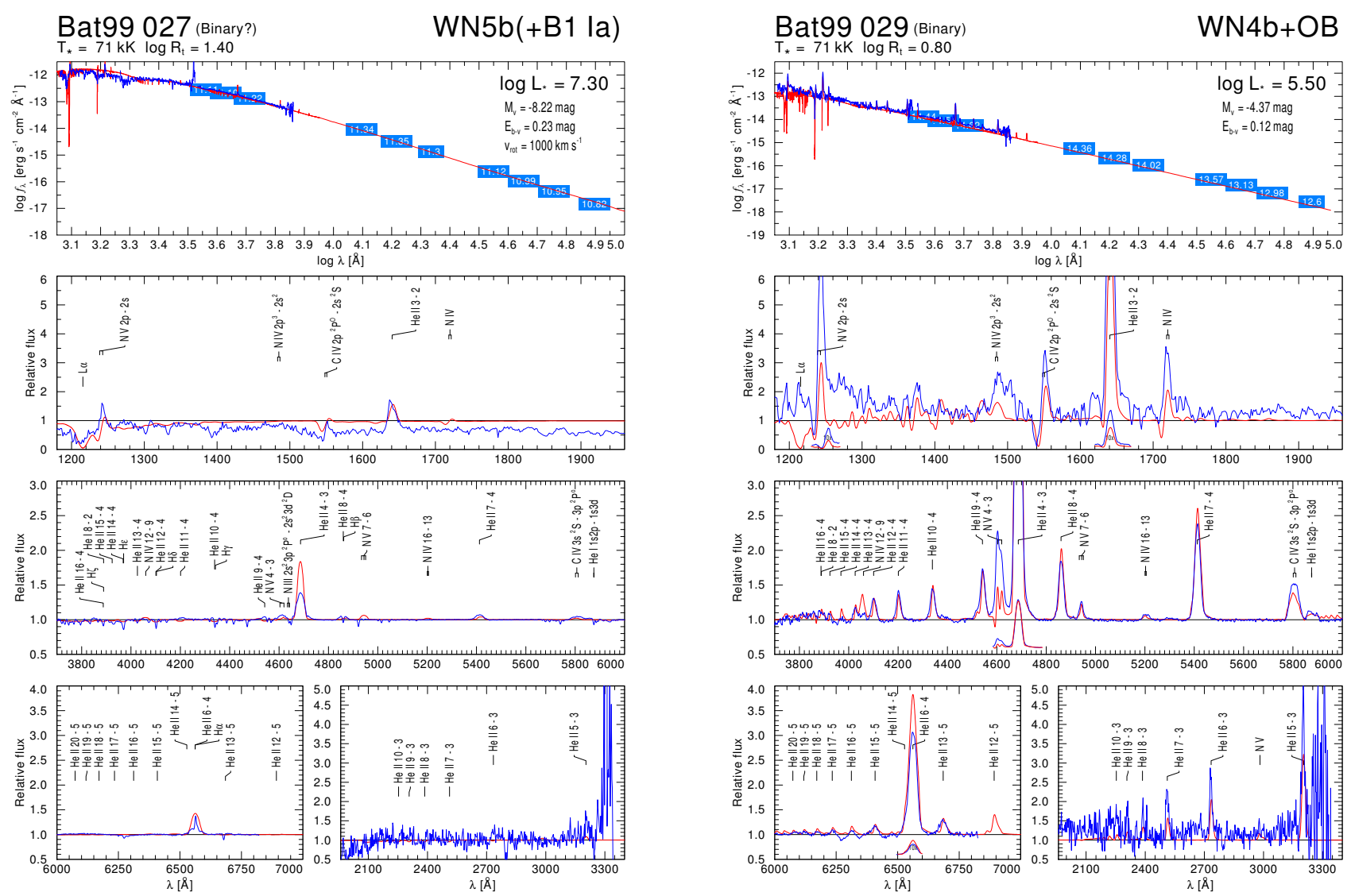

Fig. C.11. Spectral fit for BAT99 027 and BAT99 029.
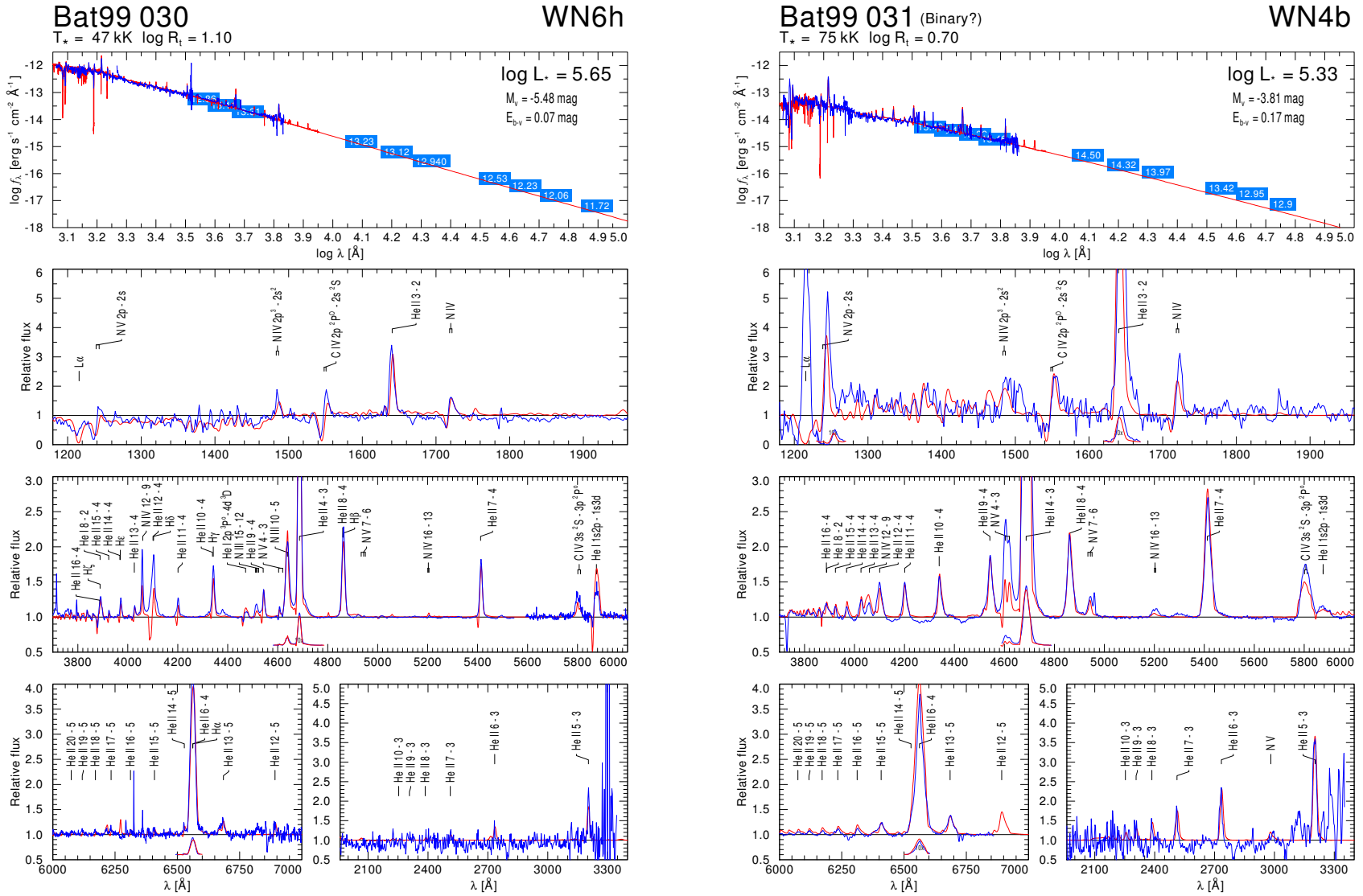

Fig. C.12. Spectral fit for BAT99 030 and BAT99 031. 

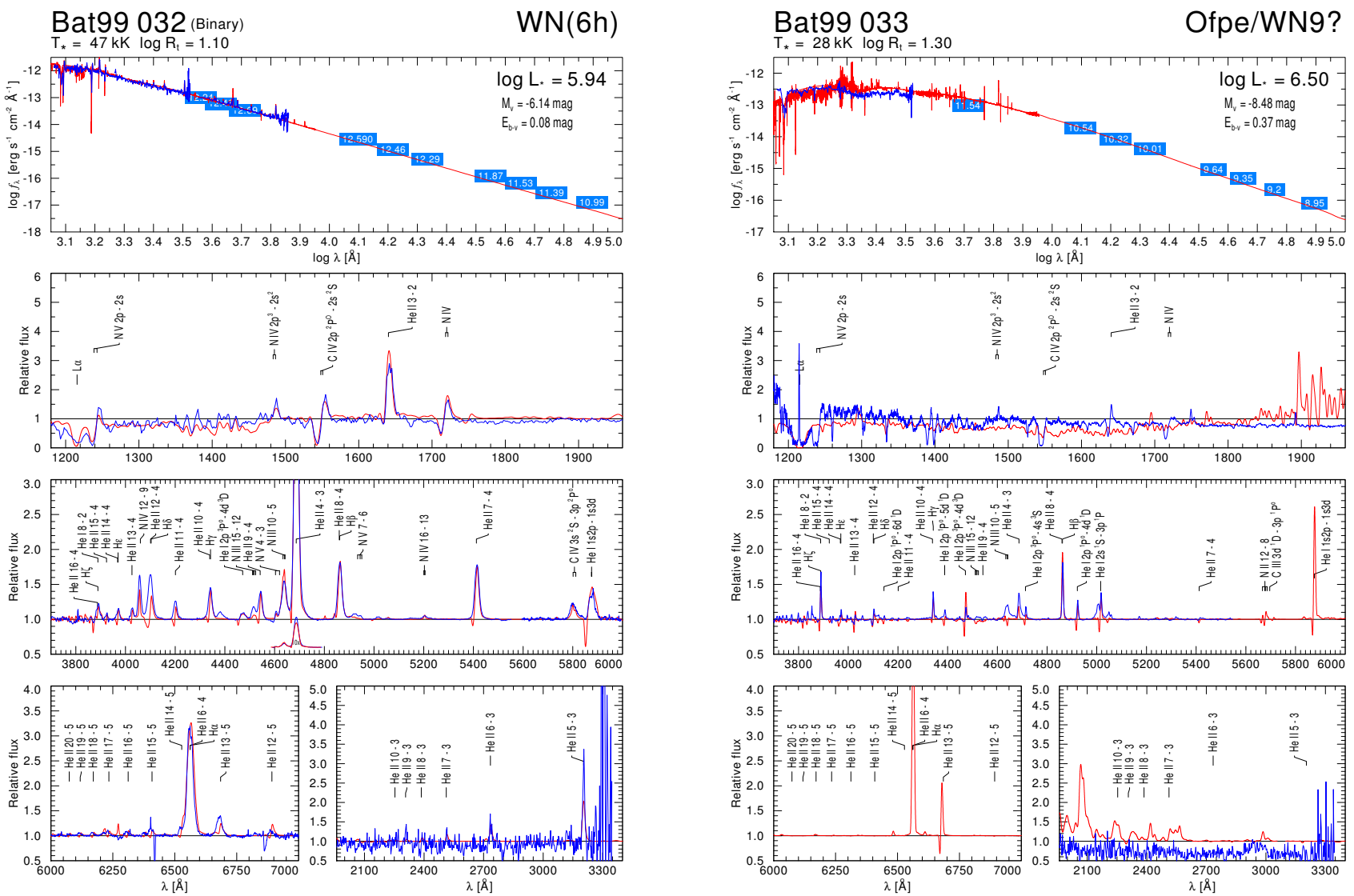

Fig. C.13. Spectral fit for BAT99 032 and BAT99 033.
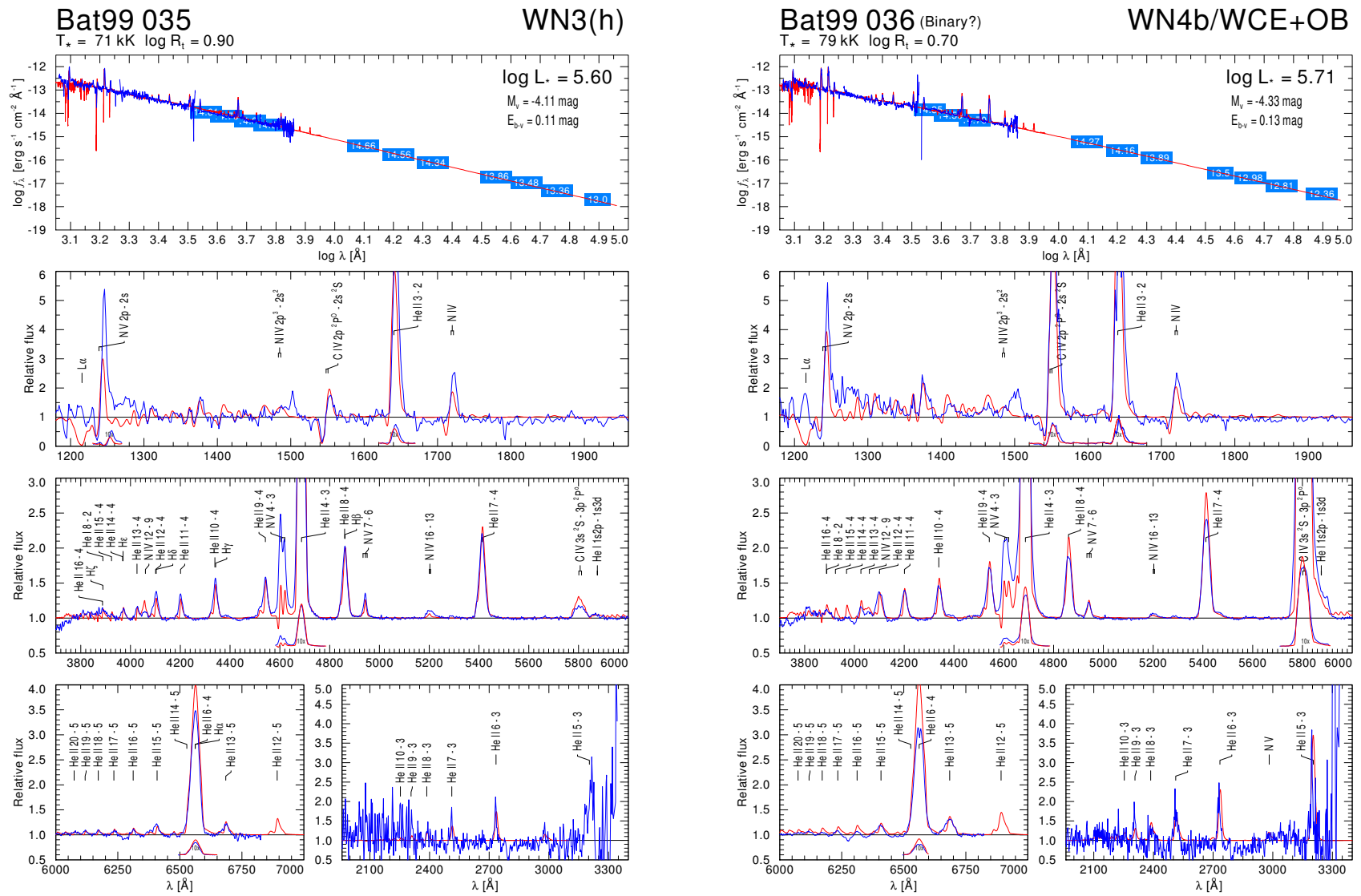

Fig. C.14. Spectral fit for BAT99 035 and BAT99 036. 
R. Hainich et al.: The Wolf-Rayet stars in the Large Magellanic Cloud
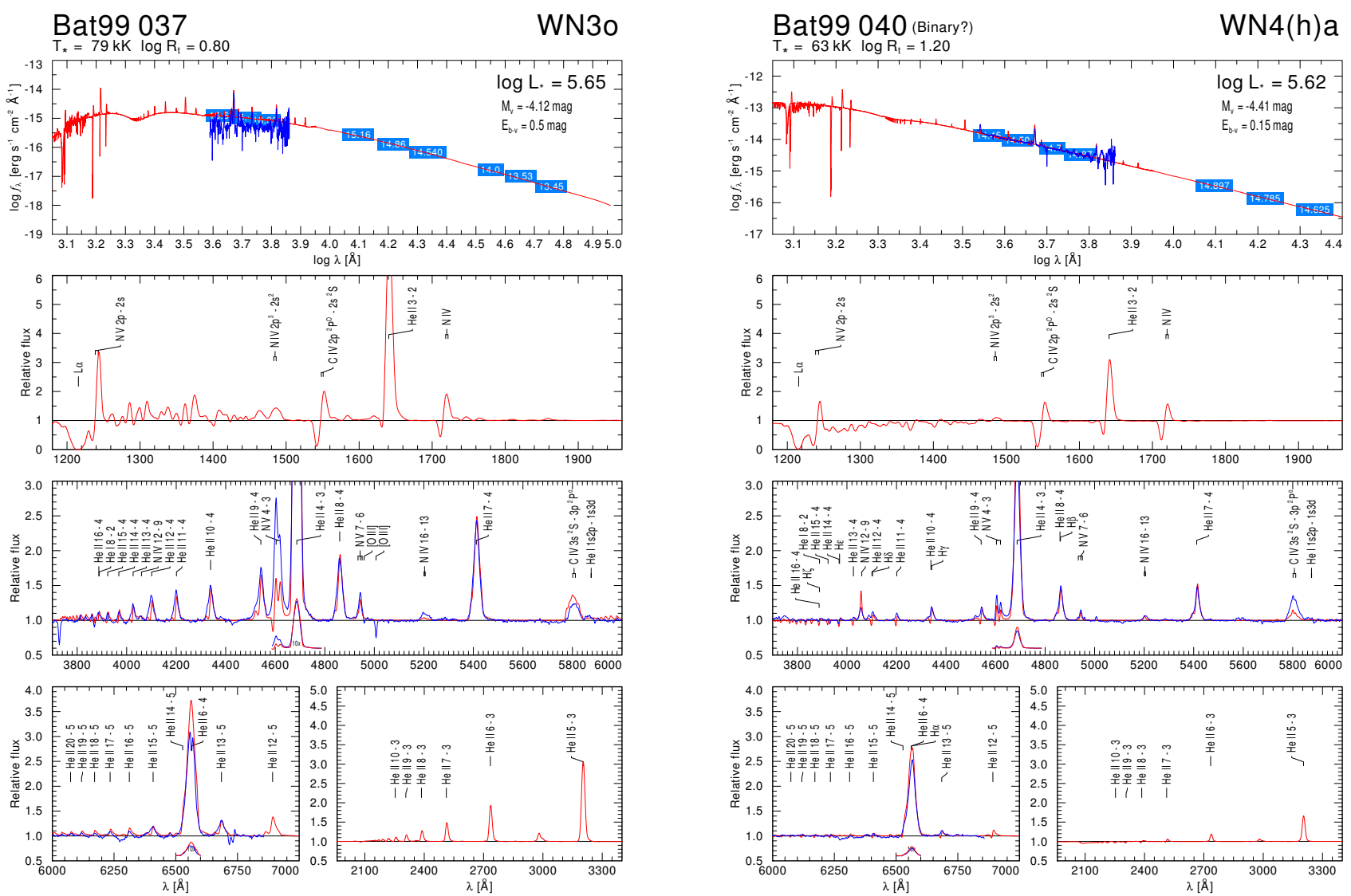

Fig. C.15. Spectral fit for BAT99 037 and BAT99 040.
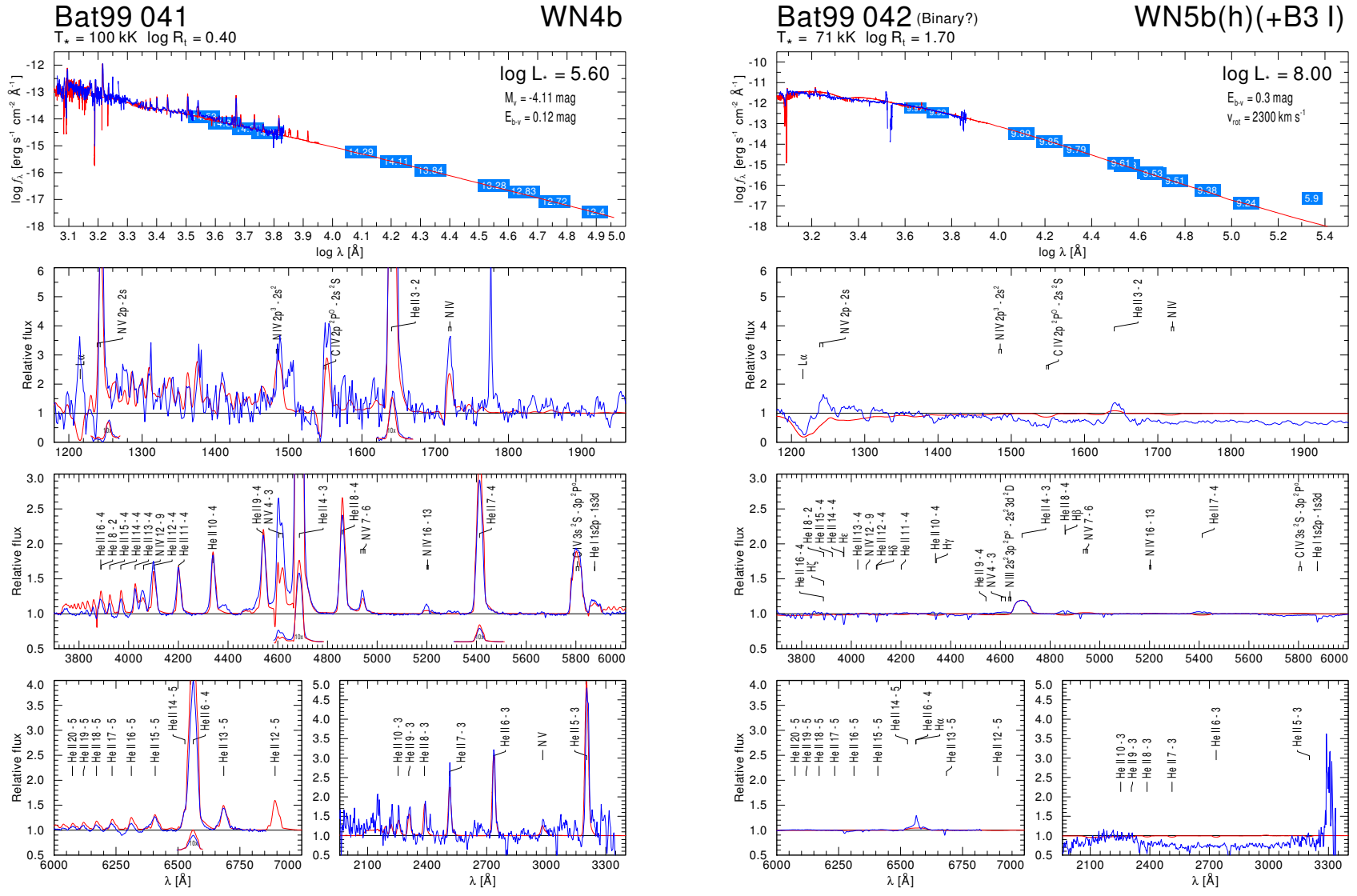

Fig. C.16. Spectral fit for BAT99 041 and BAT99 042. 


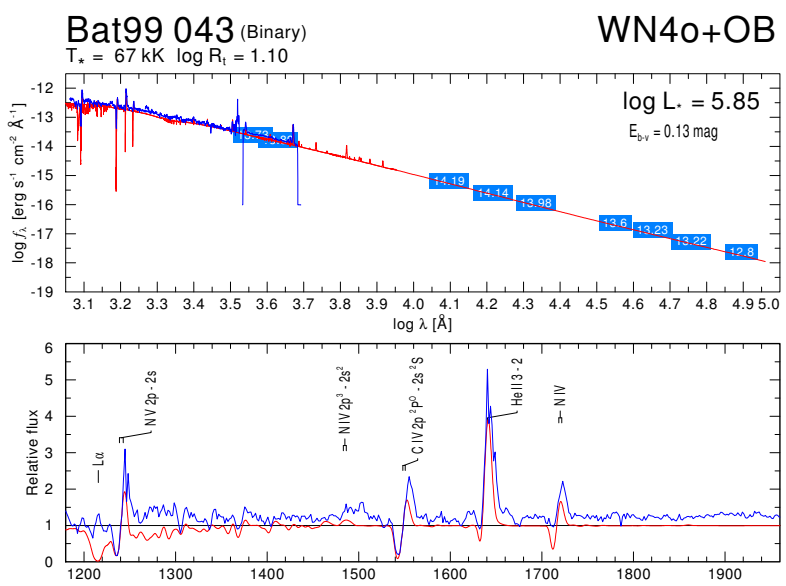

Bat99 044

WN8ha
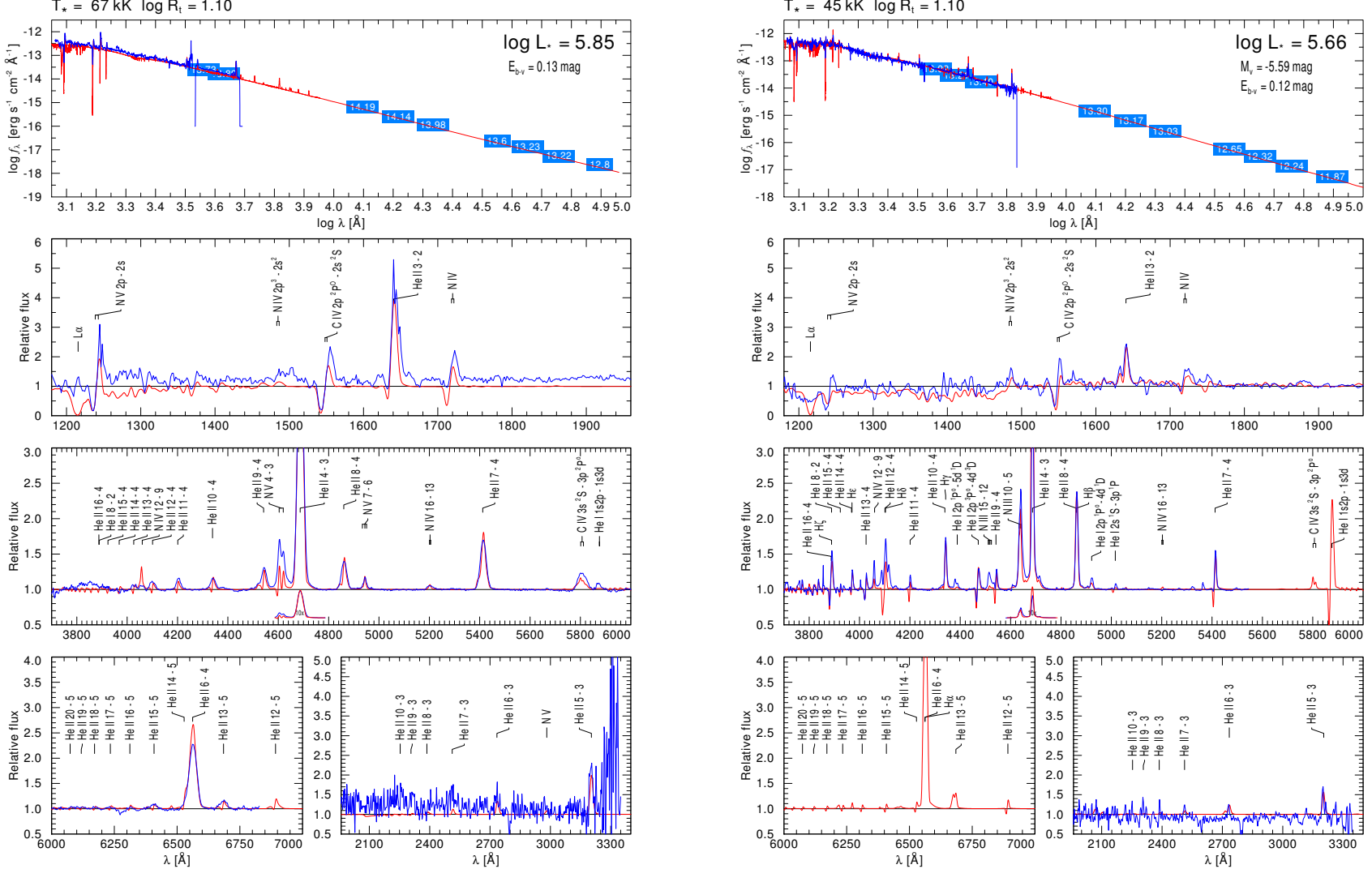

Fig. C.17. Spectral fit for BAT99 043 and BAT99 044.
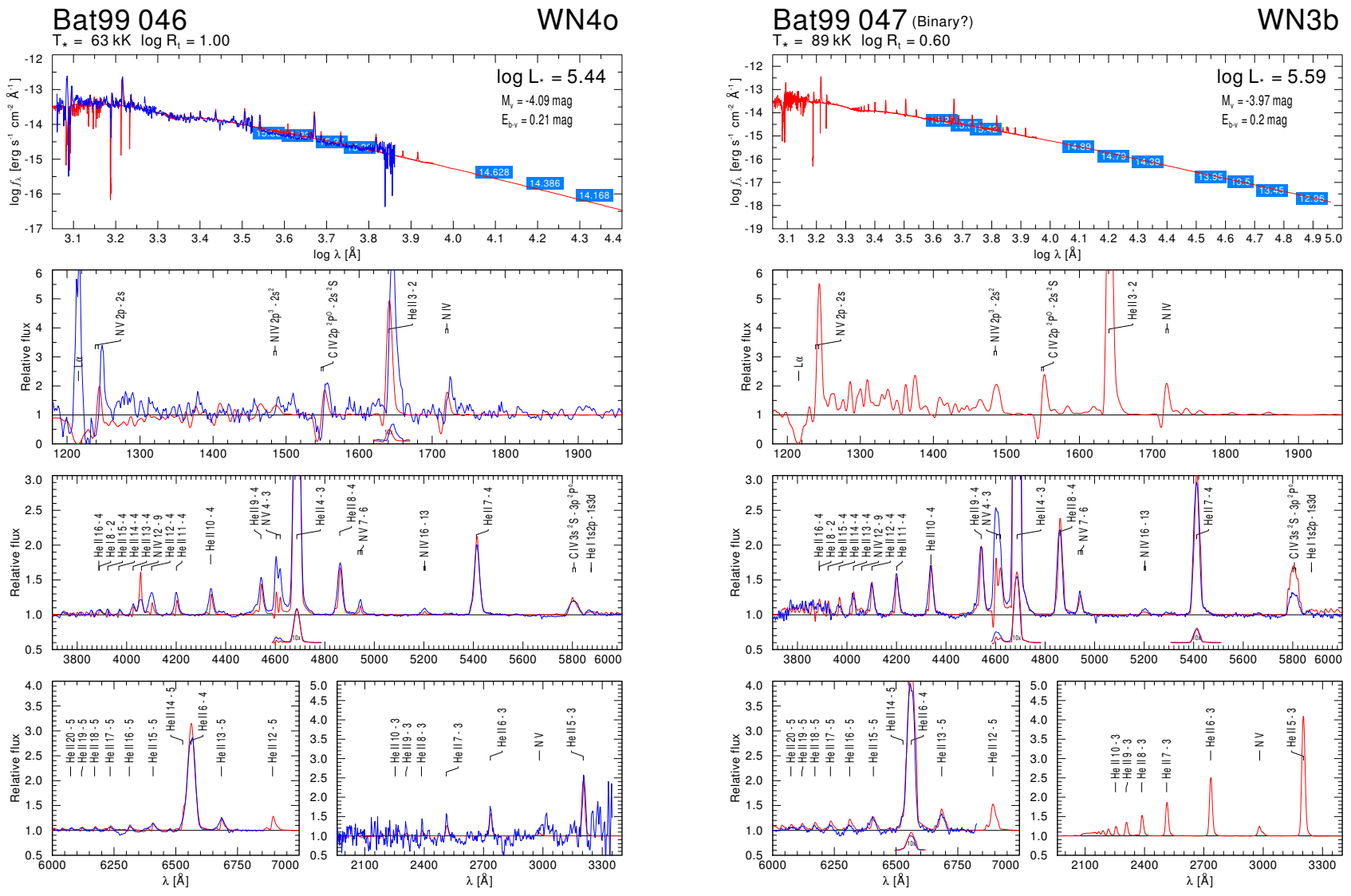

Fig. C.18. Spectral fit for BAT99 046 and BAT99 047. 
R. Hainich et al.: The Wolf-Rayet stars in the Large Magellanic Cloud
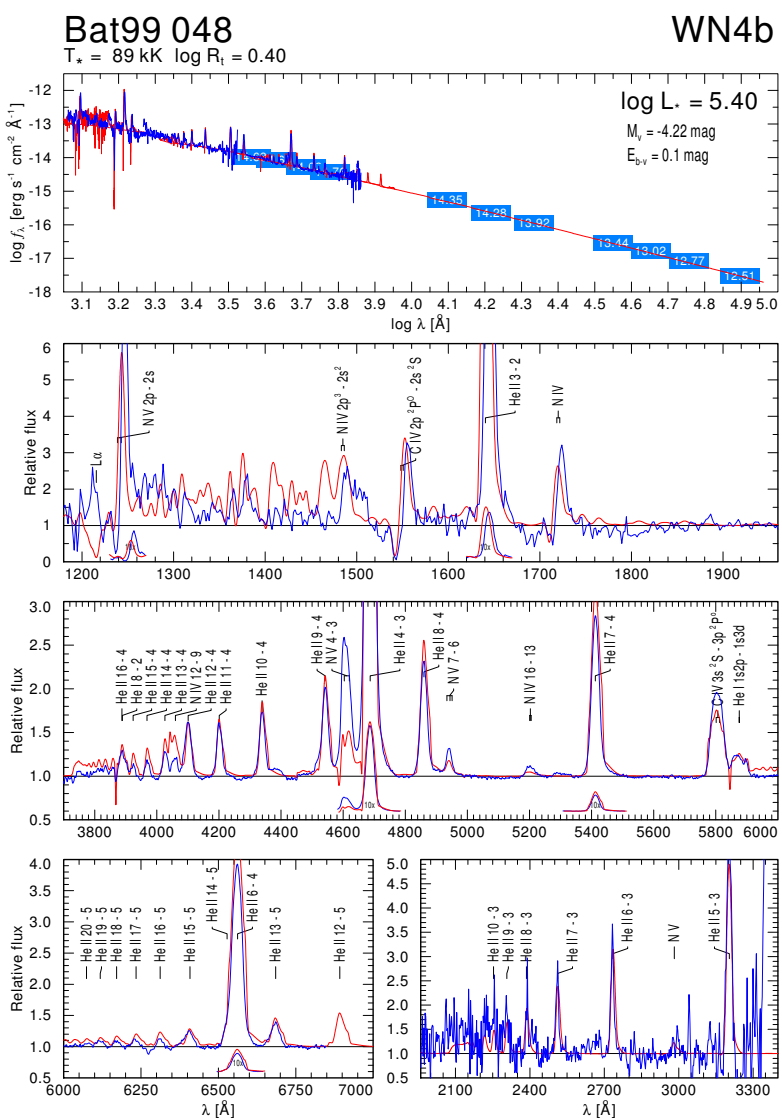

Bat99 049 (Binary)

WN4:b+O8 V
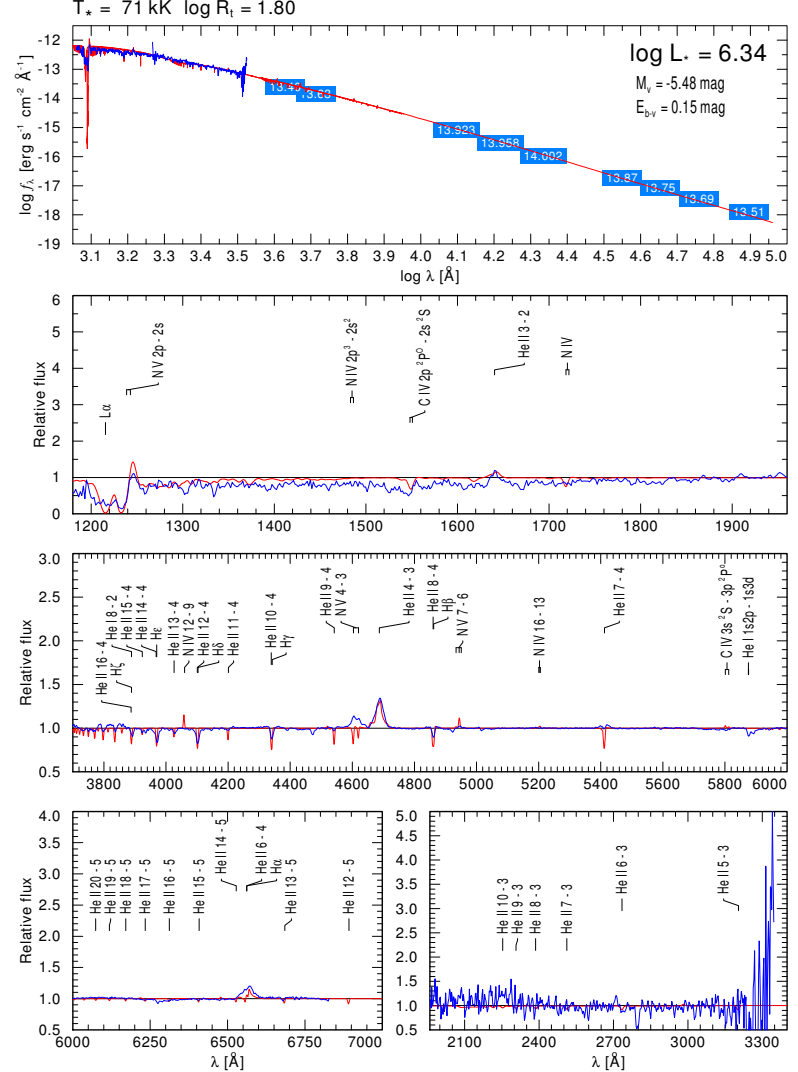

Fig. C.19. Spectral fit for BAT99 048 and BAT99 049.
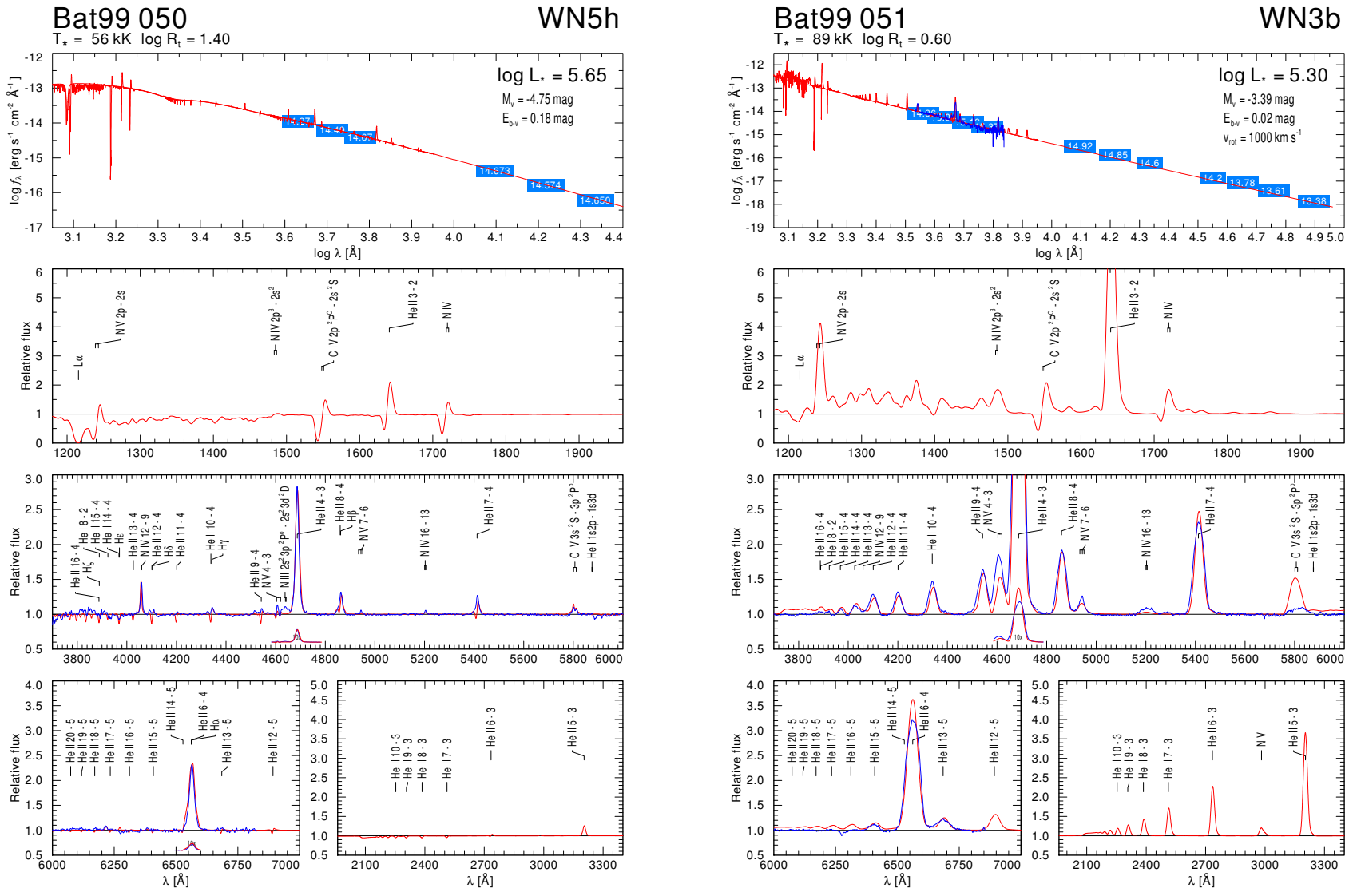

Fig. C.20. Spectral fit for BAT99 050 and BAT99 051. 
Bat99 054
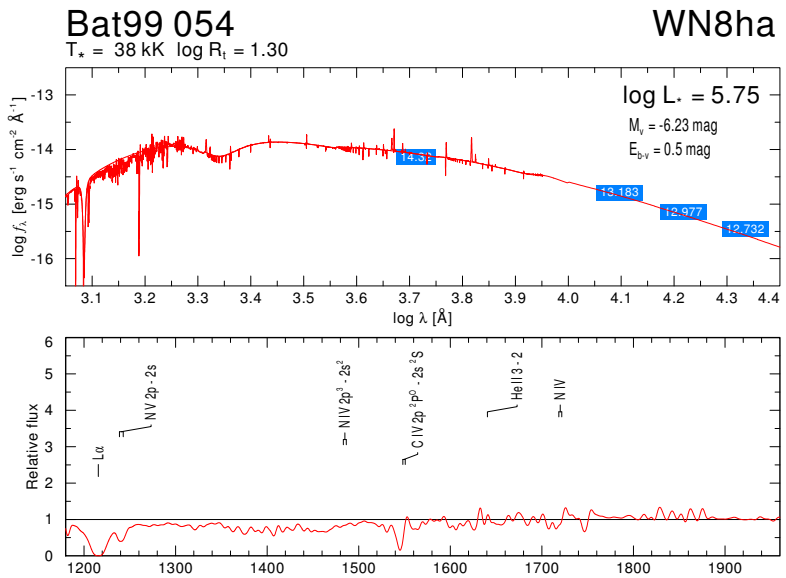

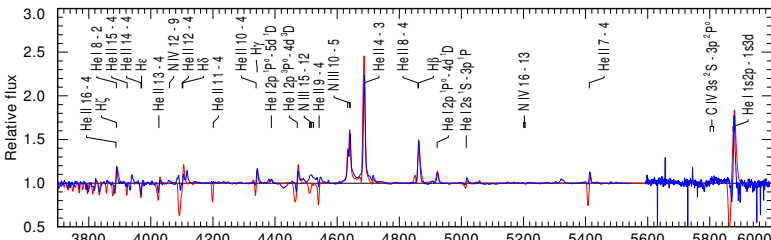

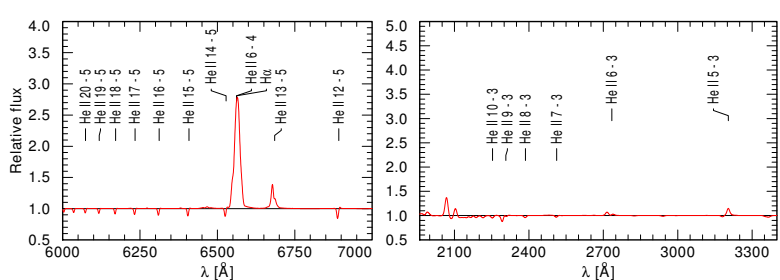

Fig. C.21. Spectral fit for BAT99 054 and BAT99 055.
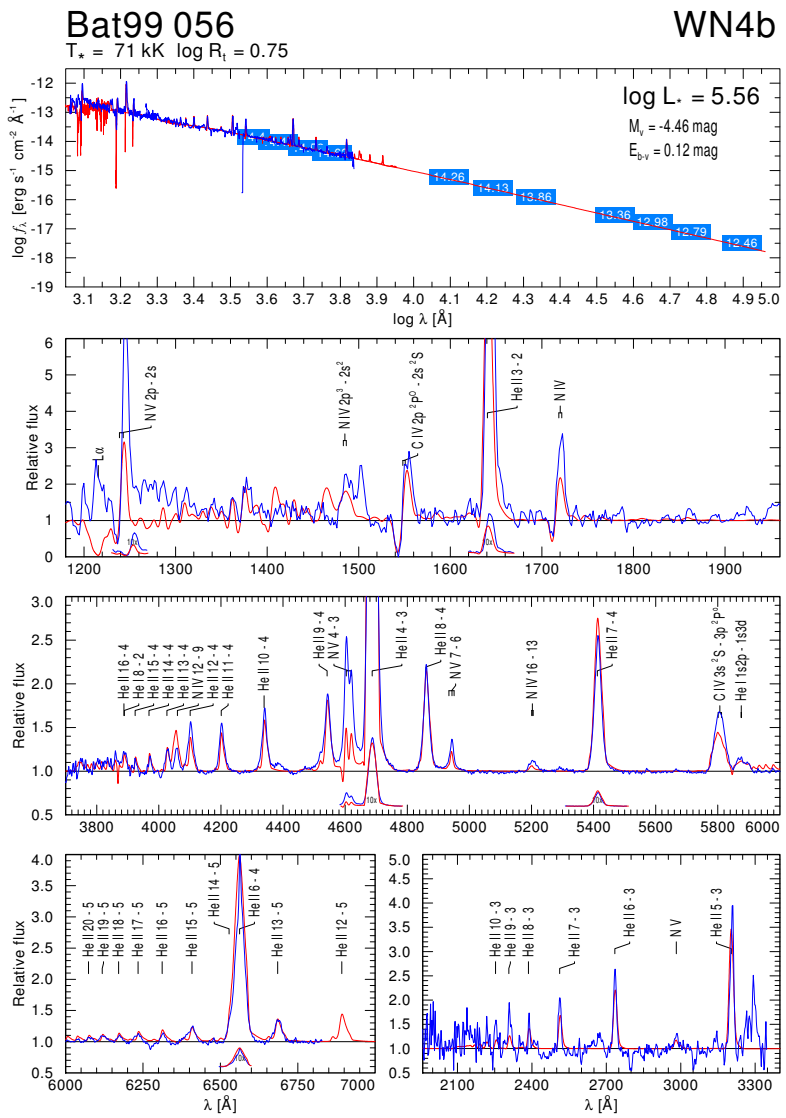

Fig. C.22. Spectral fit for BAT99 056 and BAT99 057 .
Bat99 055

WN11h
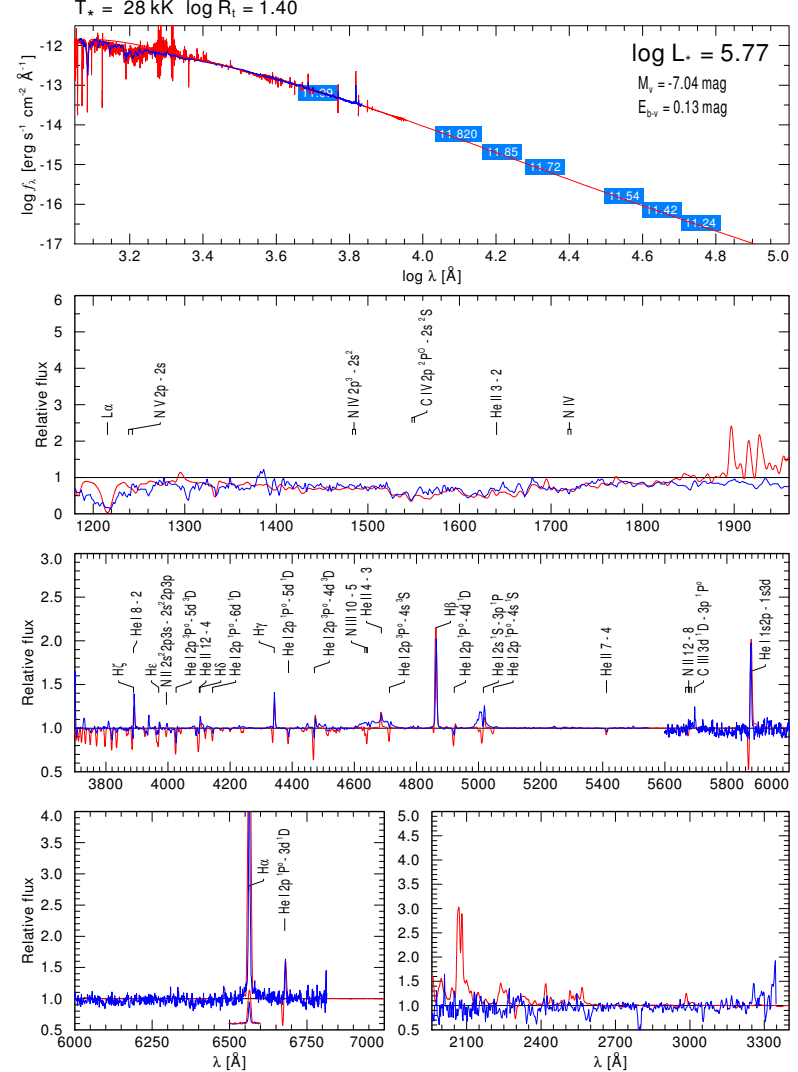
R. Hainich et al.: The Wolf-Rayet stars in the Large Magellanic Cloud

Bat99 058
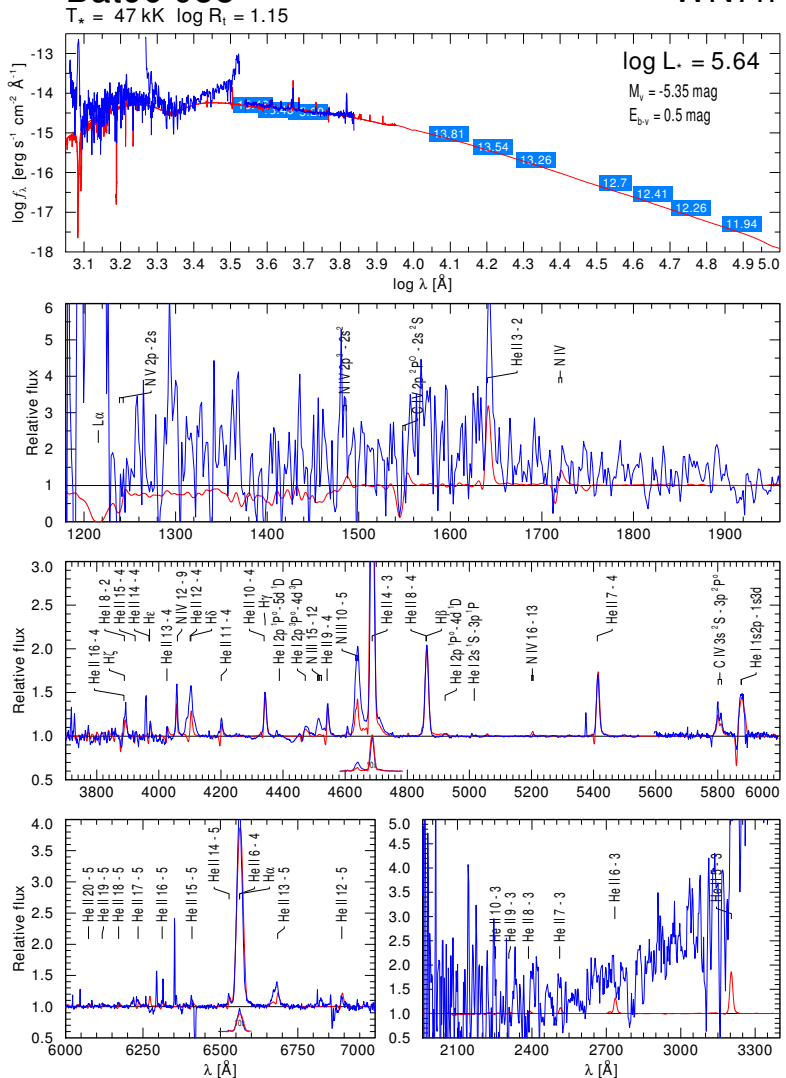

Fig. C.23. Spectral fit for BAT99 058 and BAT99 059.
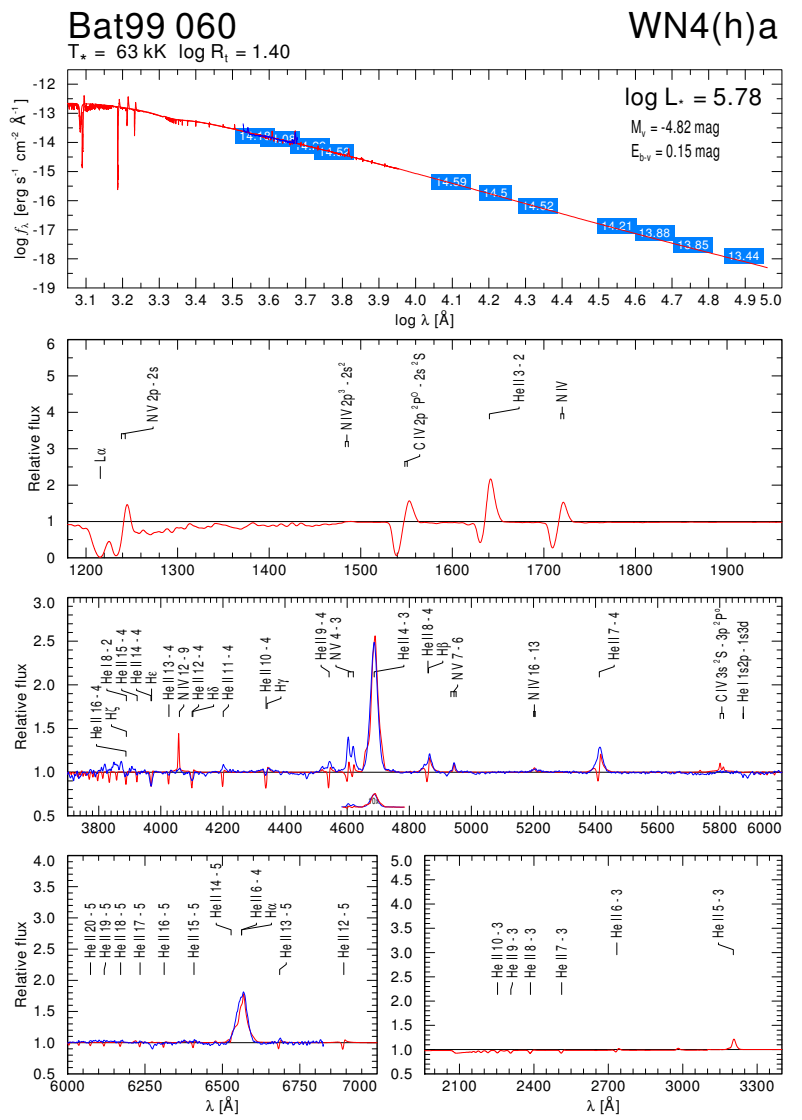

Bat99 059 (Binary?)
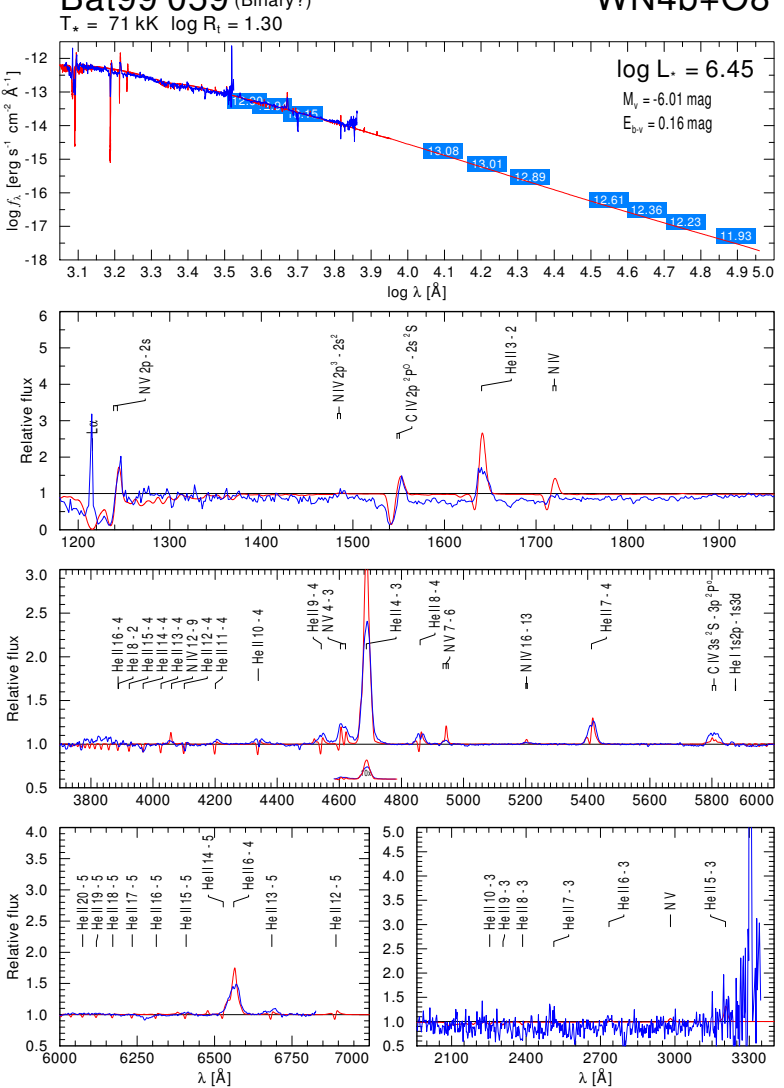

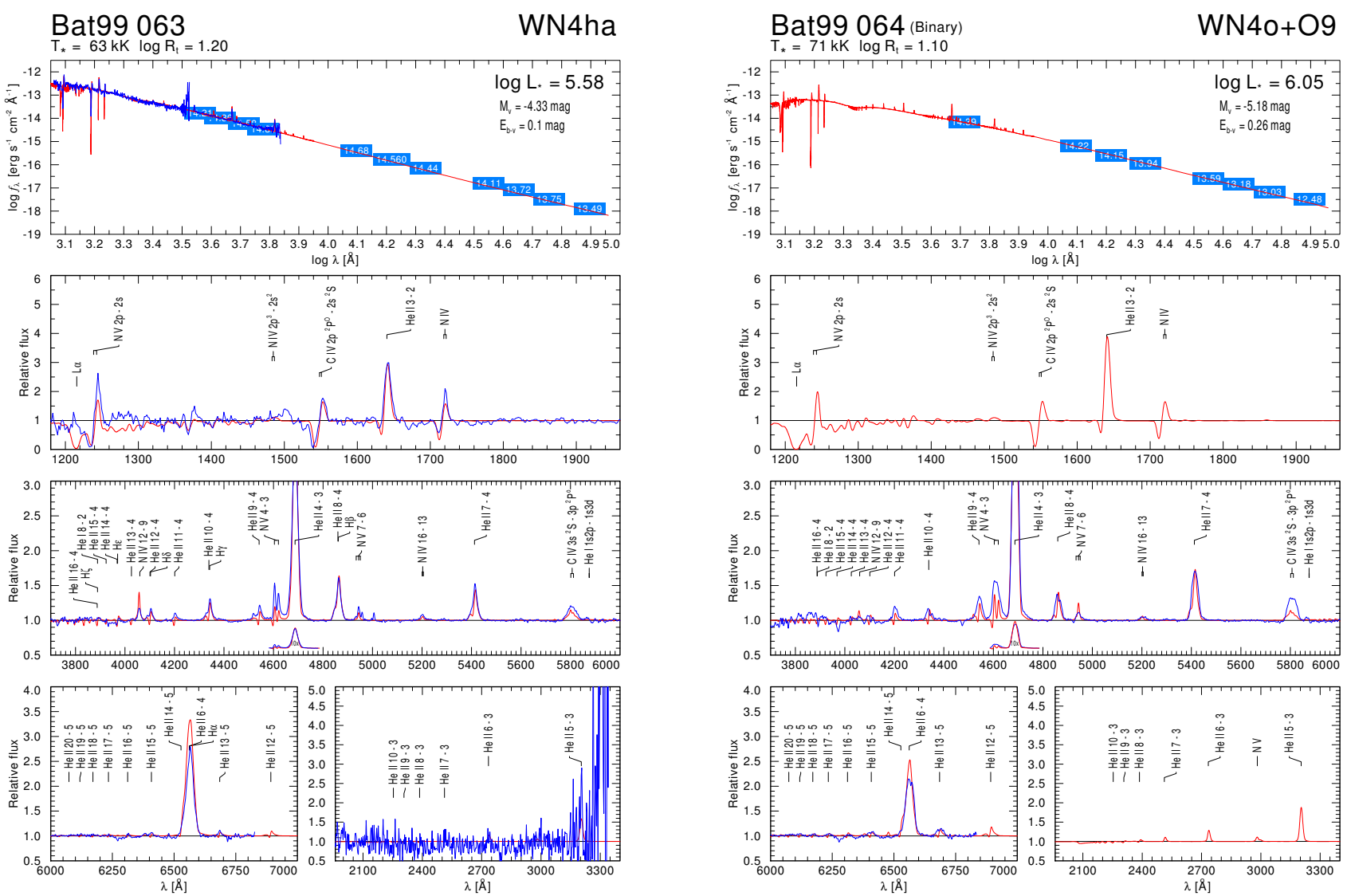

Fig. C.25. Spectral fit for BAT99 063 and BAT99 064 .
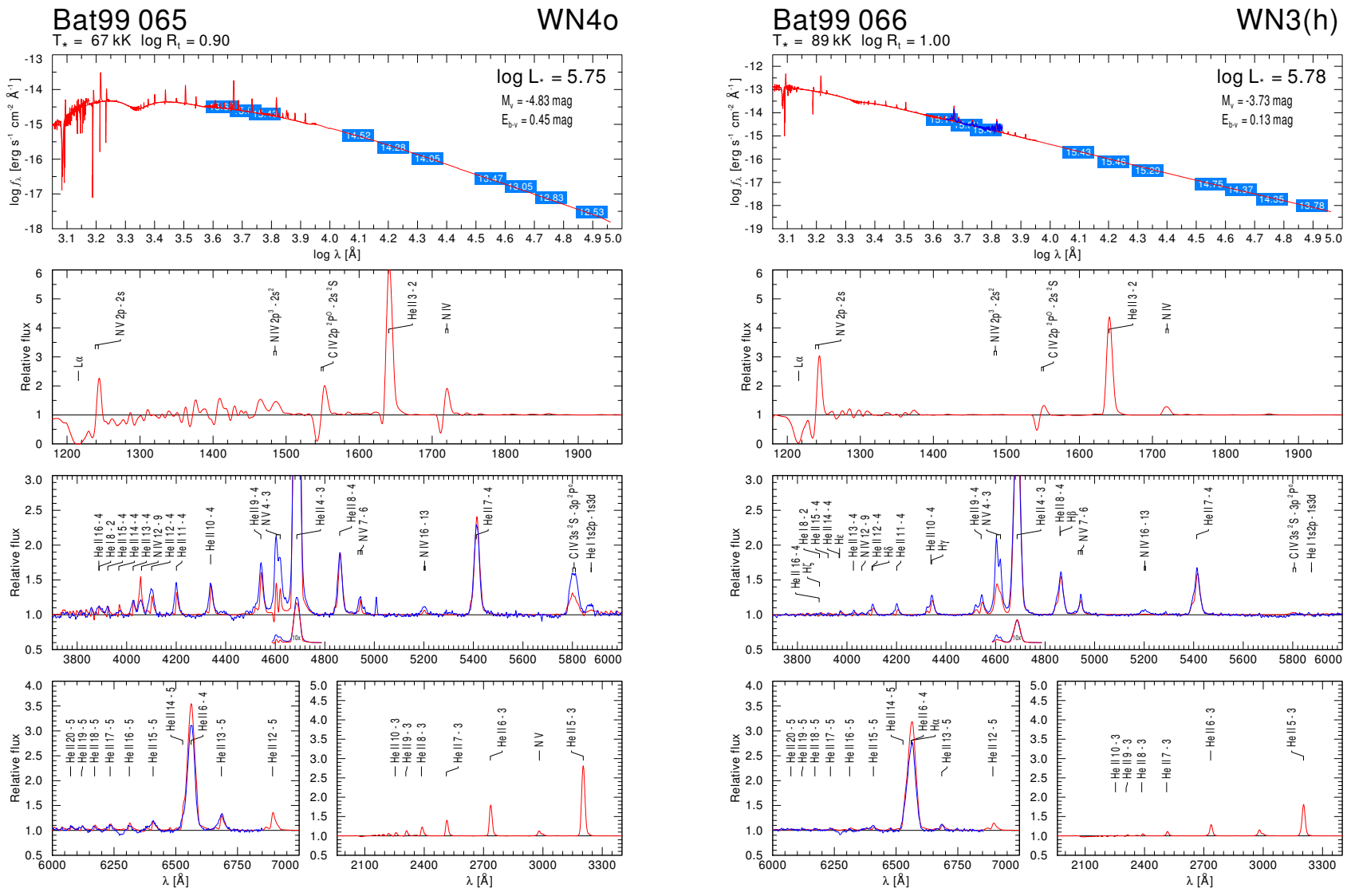

Fig. C.26. Spectral fit for BAT99 065 and BAT99 066. 
R. Hainich et al.: The Wolf-Rayet stars in the Large Magellanic Cloud
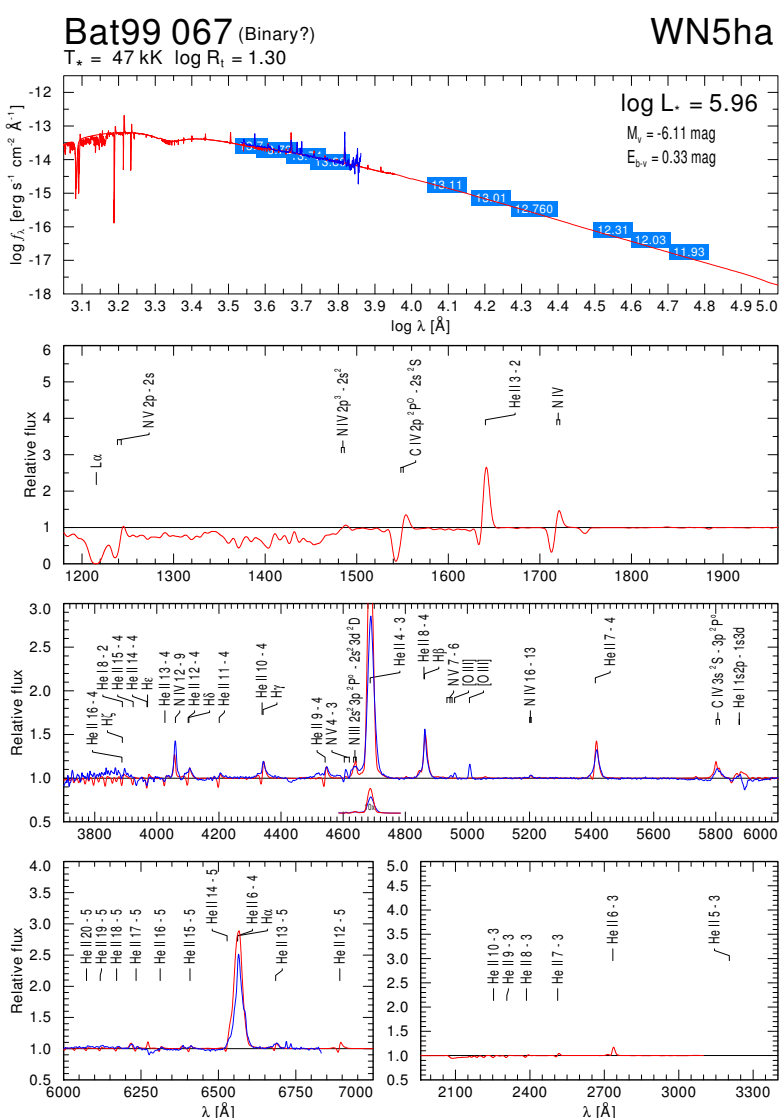

Bat99 068

O3.5 If*/WN7h
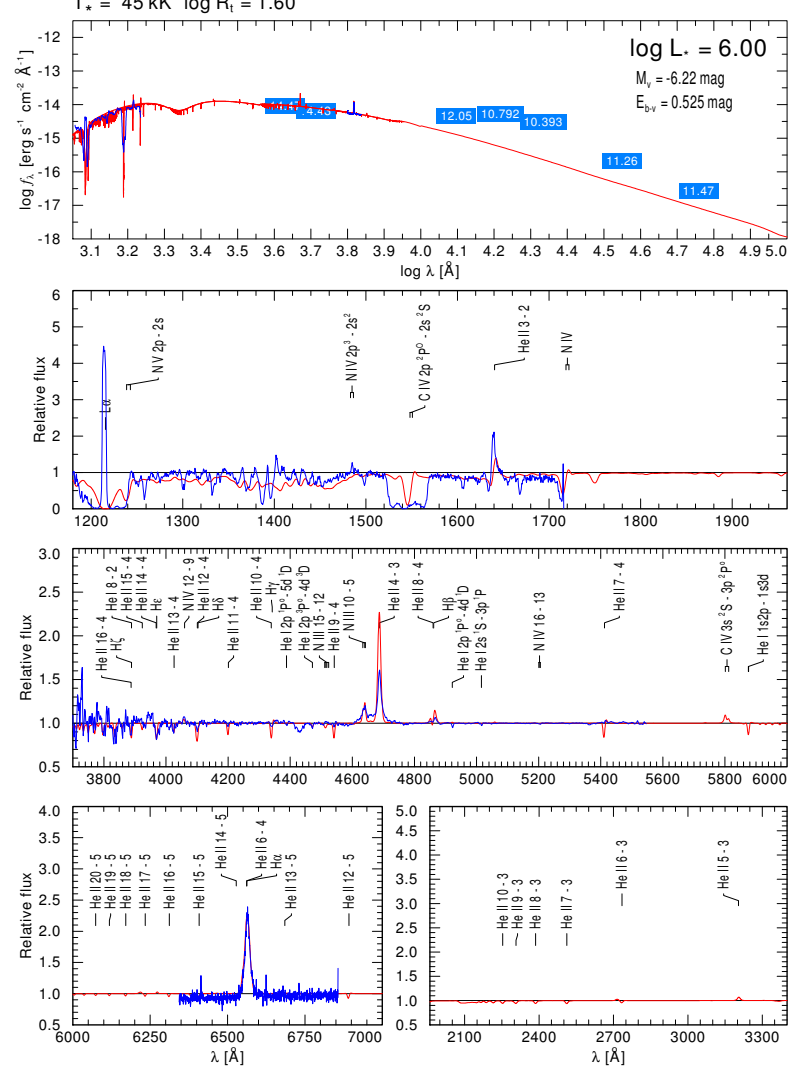

Fig. C.27. Spectral fit for BAT99 067 and BAT99 068 .
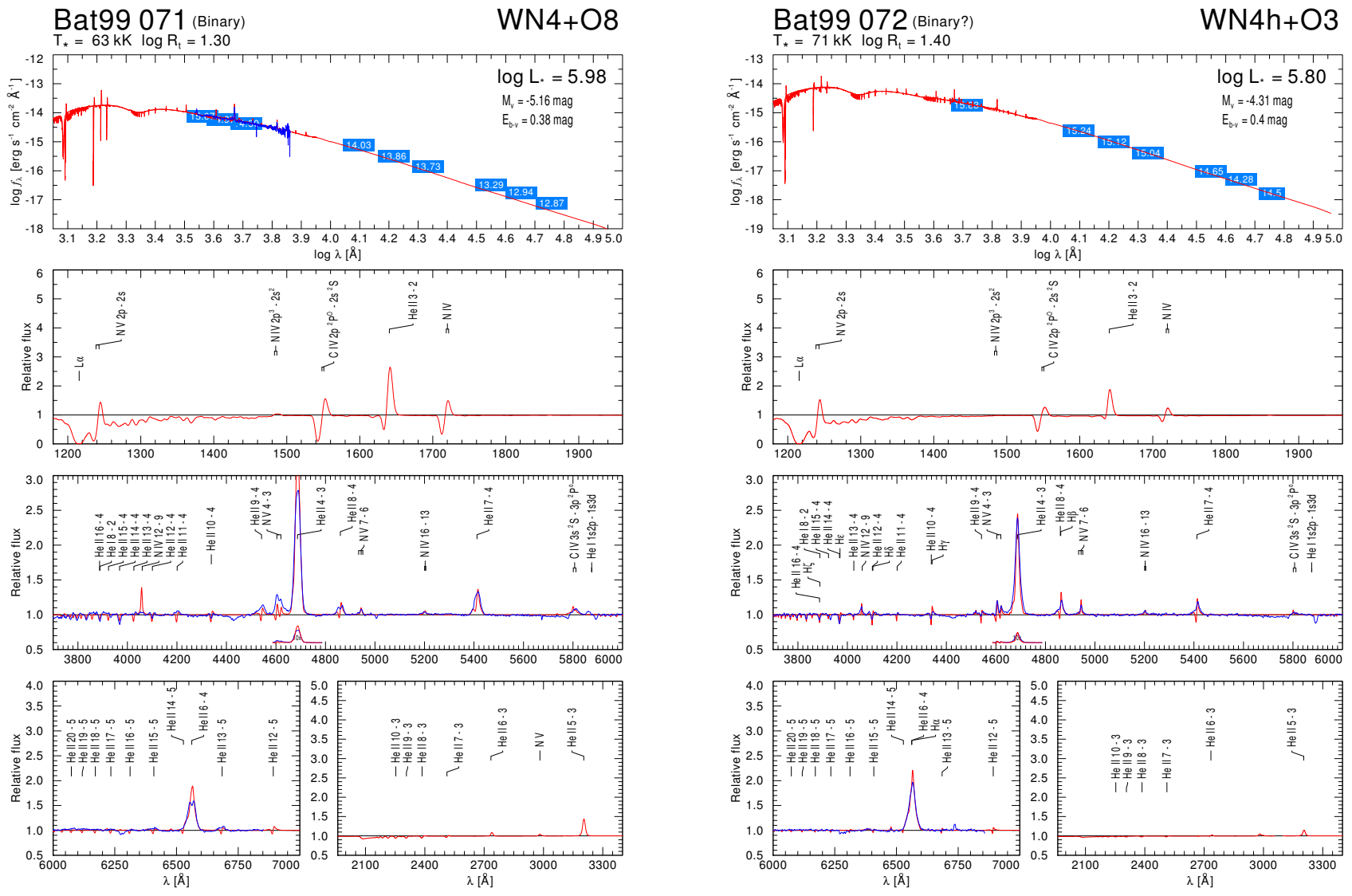

Fig. C.28. Spectral fit for BAT99 071 and BAT99 072. 
Bat99 073
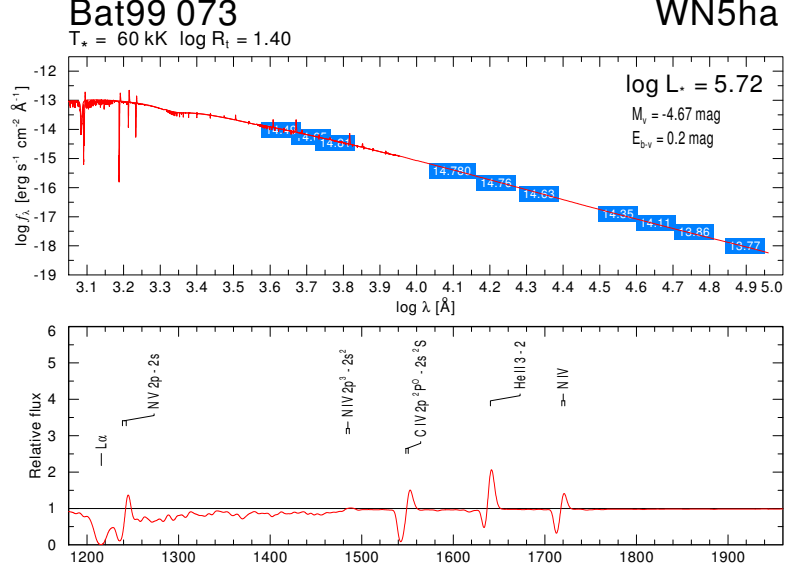

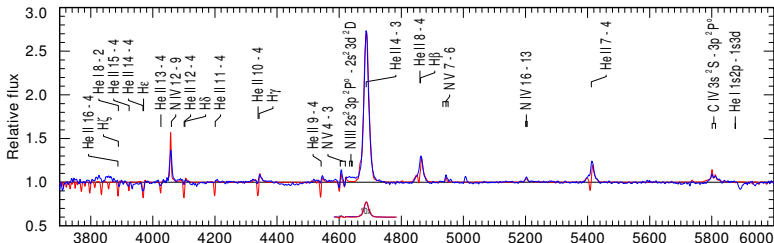

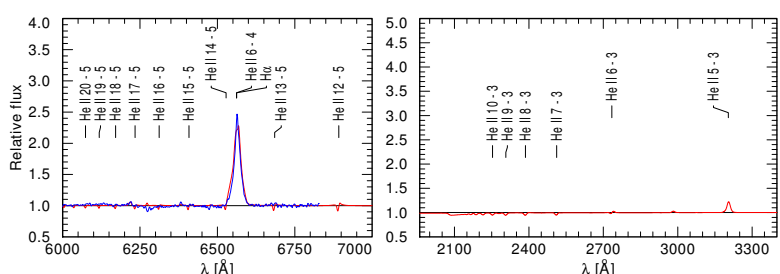

Fig. C.29. Spectral fit for BAT99 073 and BAT99 074 .
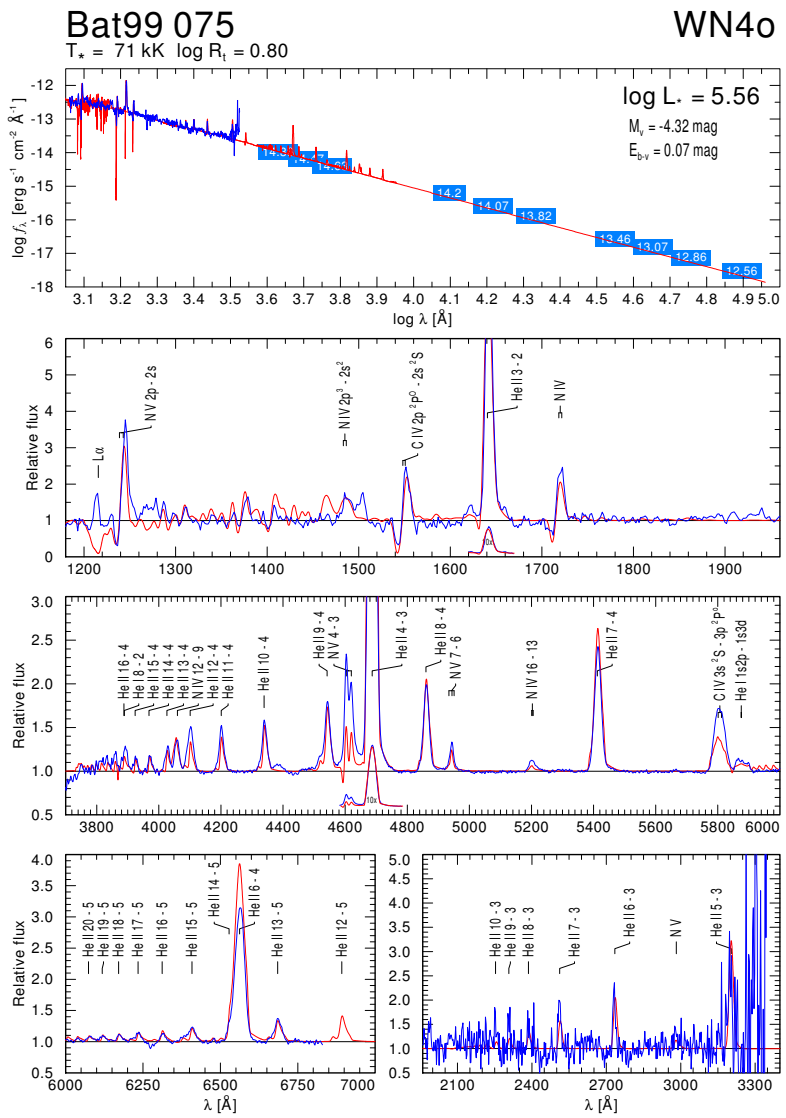

Fig. C.30. Spectral fit for BAT99 075 and BAT99 076.
Bat99 074

WN3(h)
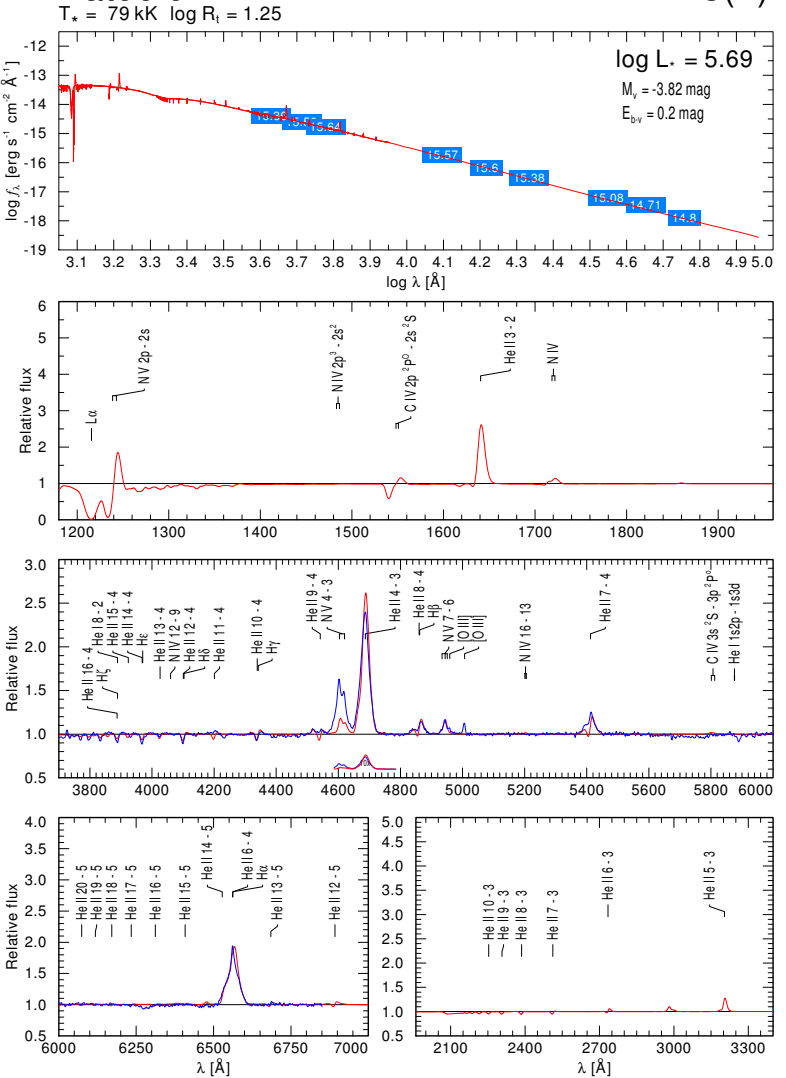
R. Hainich et al.: The Wolf-Rayet stars in the Large Magellanic Cloud
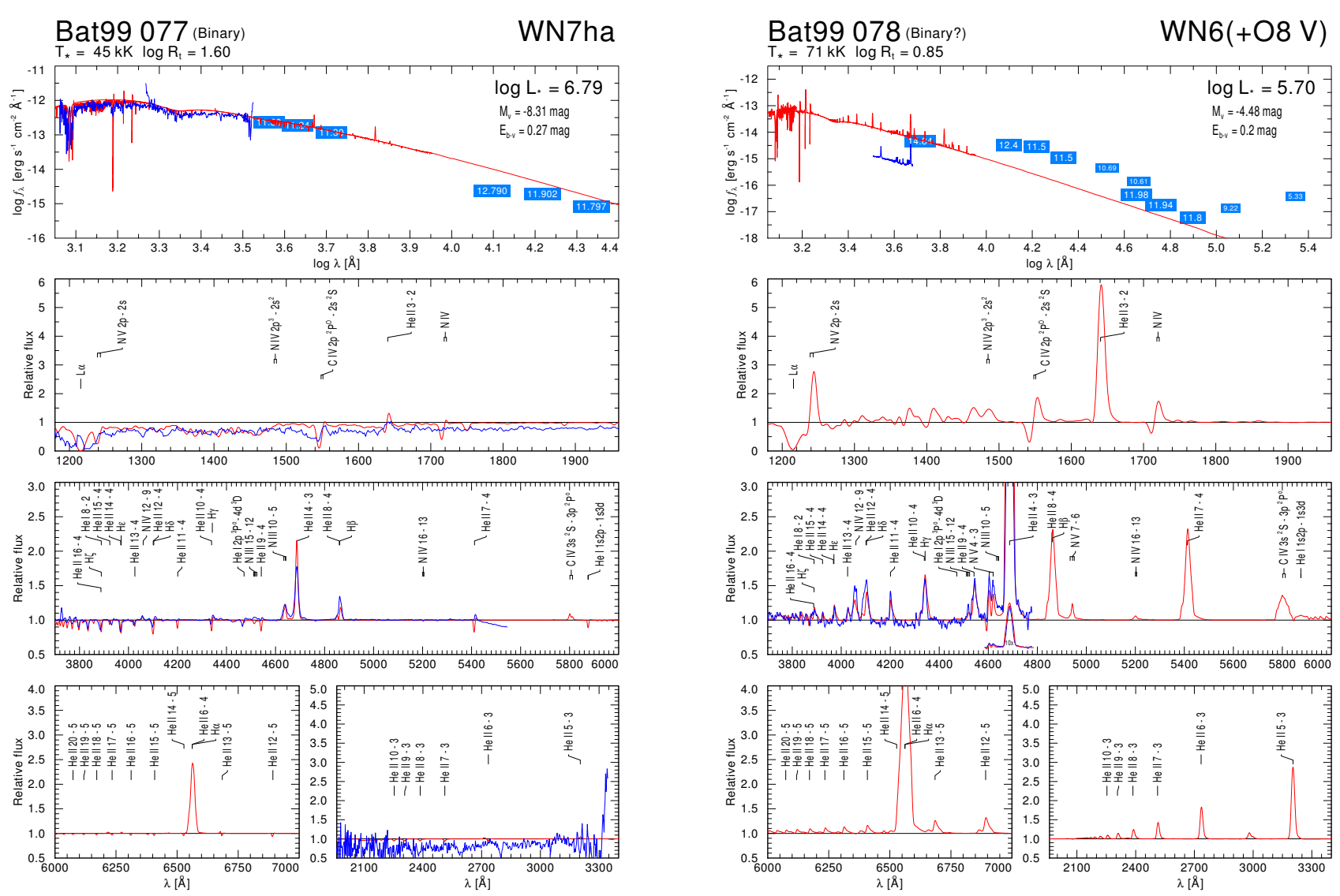

Fig. C.31. Spectral fit for BAT99 077 and BAT99 078.
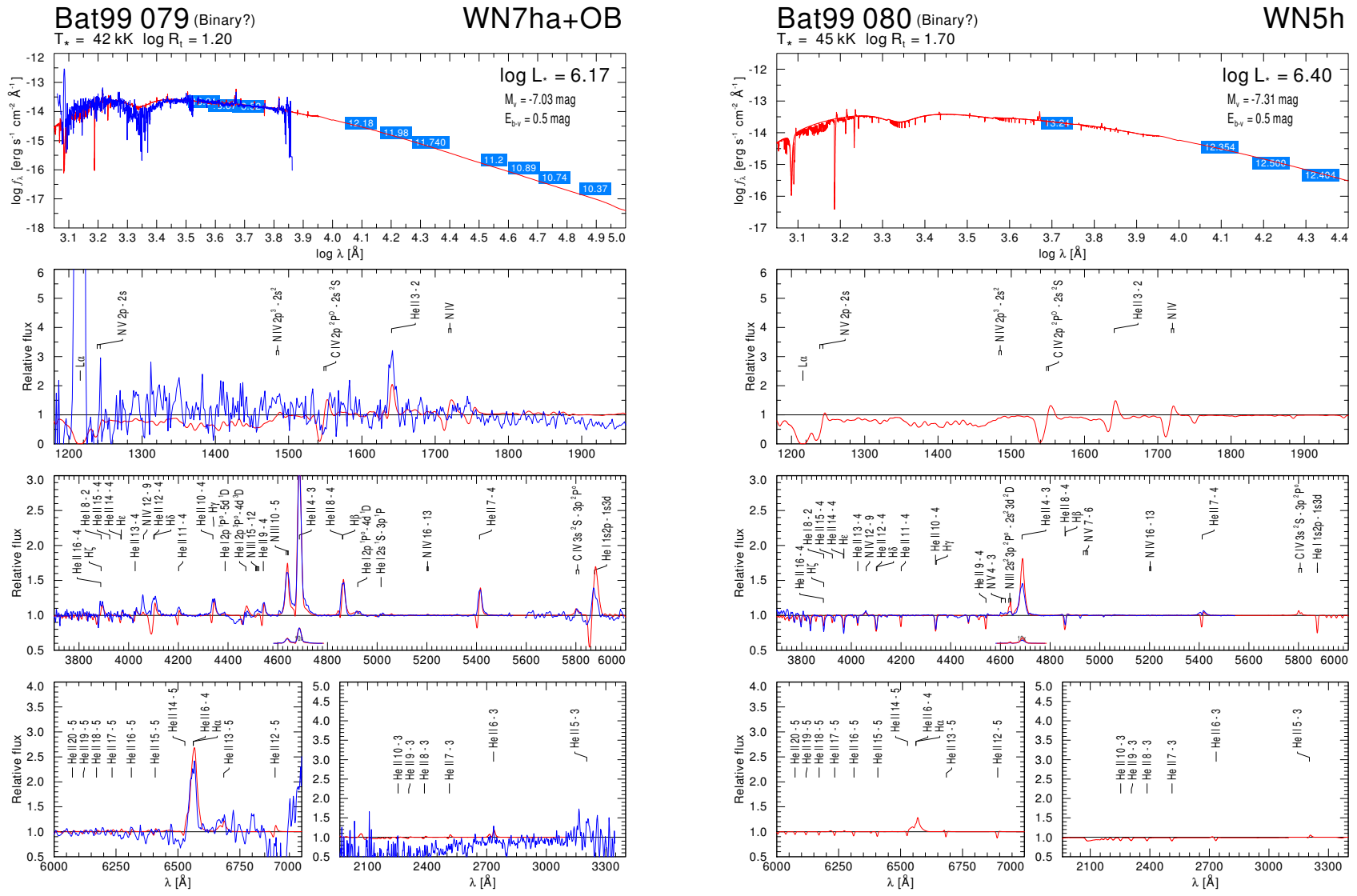

Fig. C.32. Spectral fit for BAT99 079 and BAT99 080. 
Bat99 081

WN5h
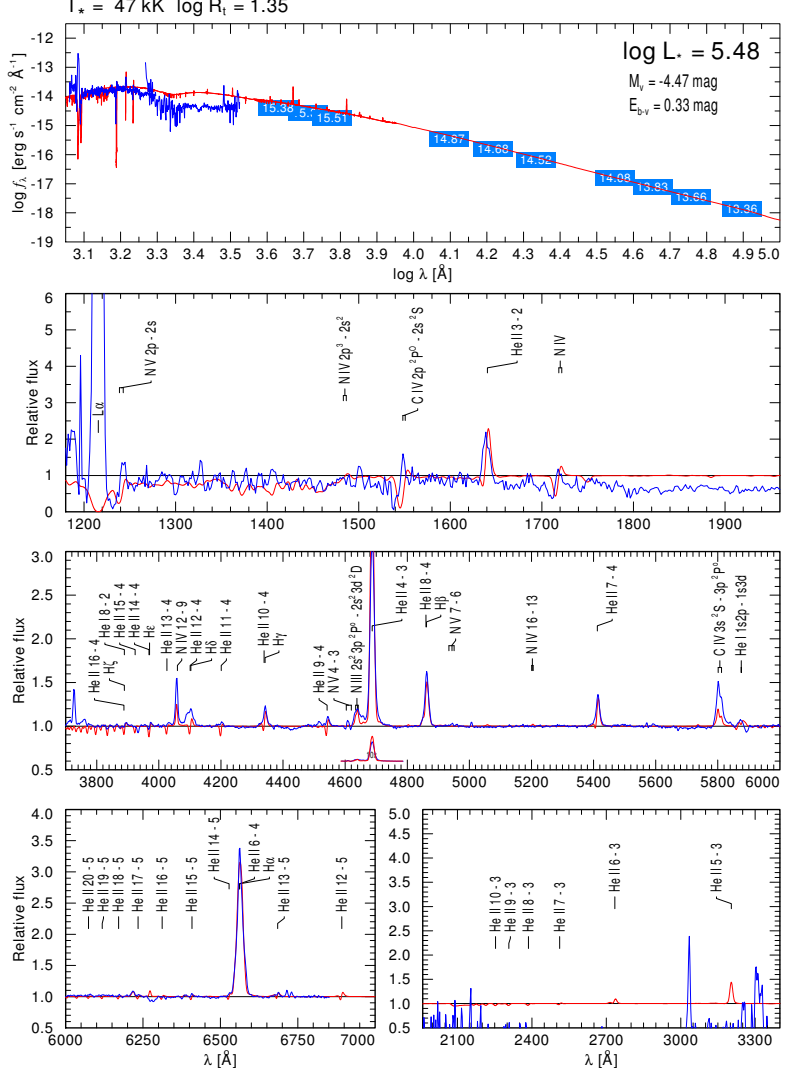

Fig. C.33. Spectral fit for BAT99 081 and BAT99 082.
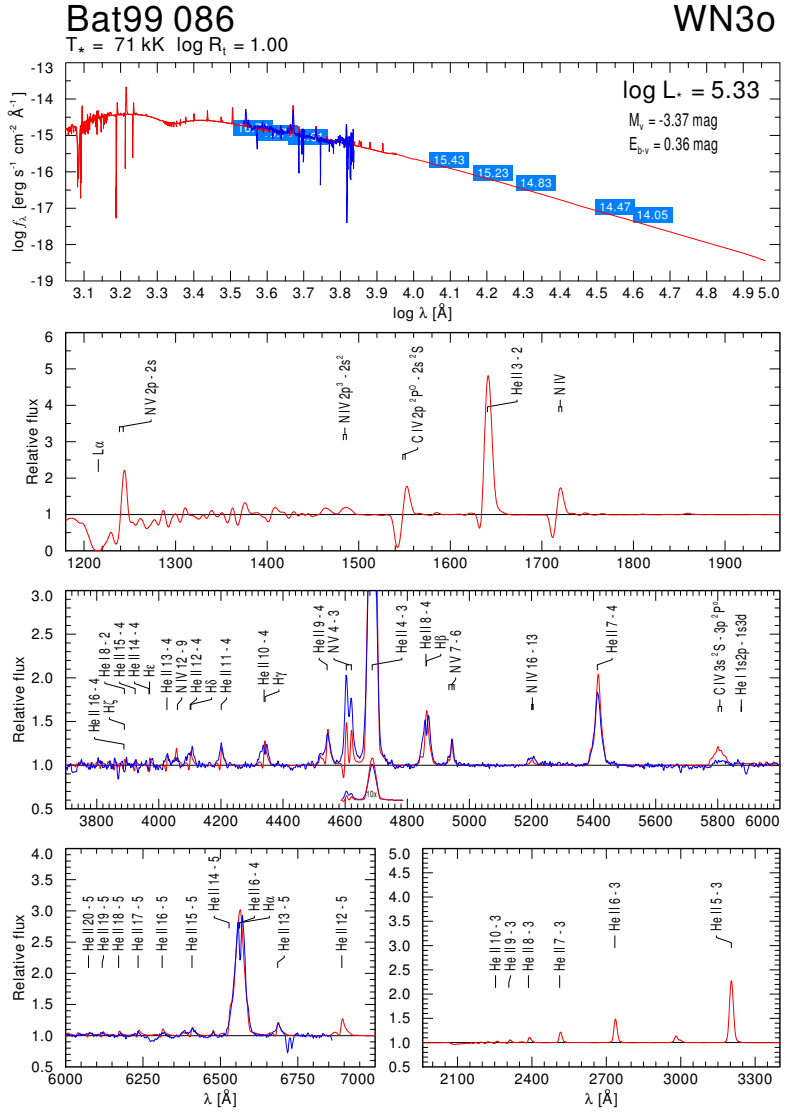

Fig. C.34. Spectral fit for BAT99 086 and BAT99 088.
Bat99 082 (Binary?)

WN3b
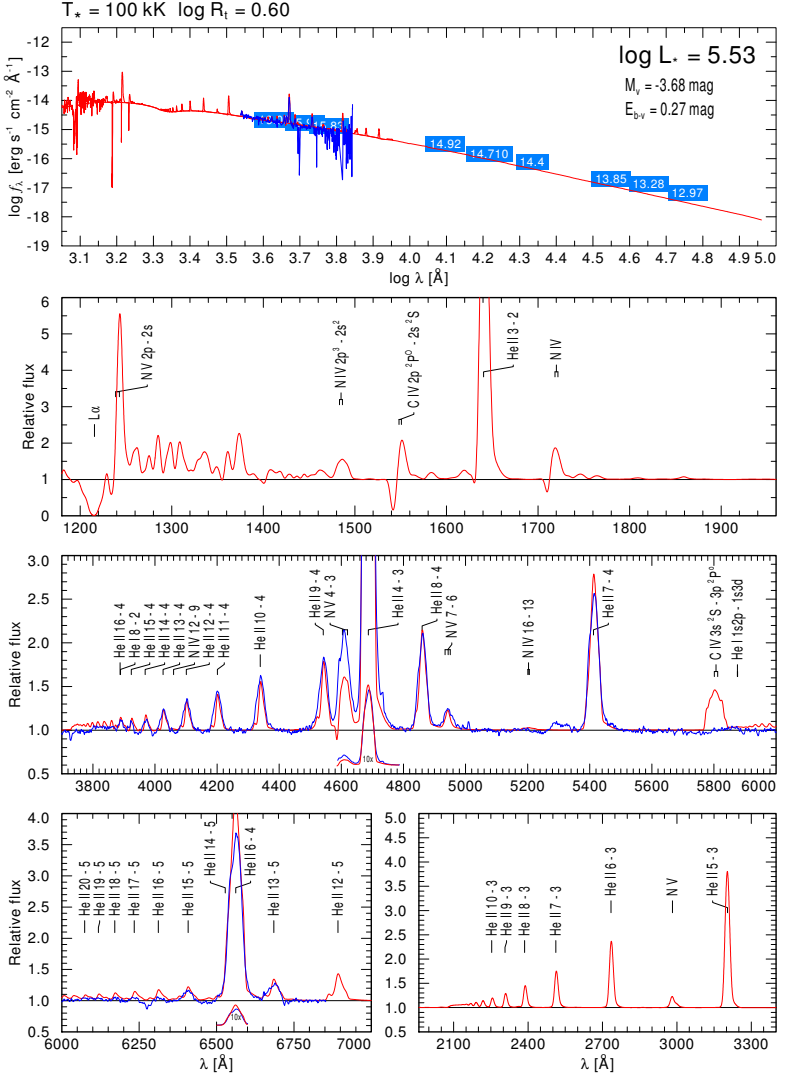

WN4b/WCE
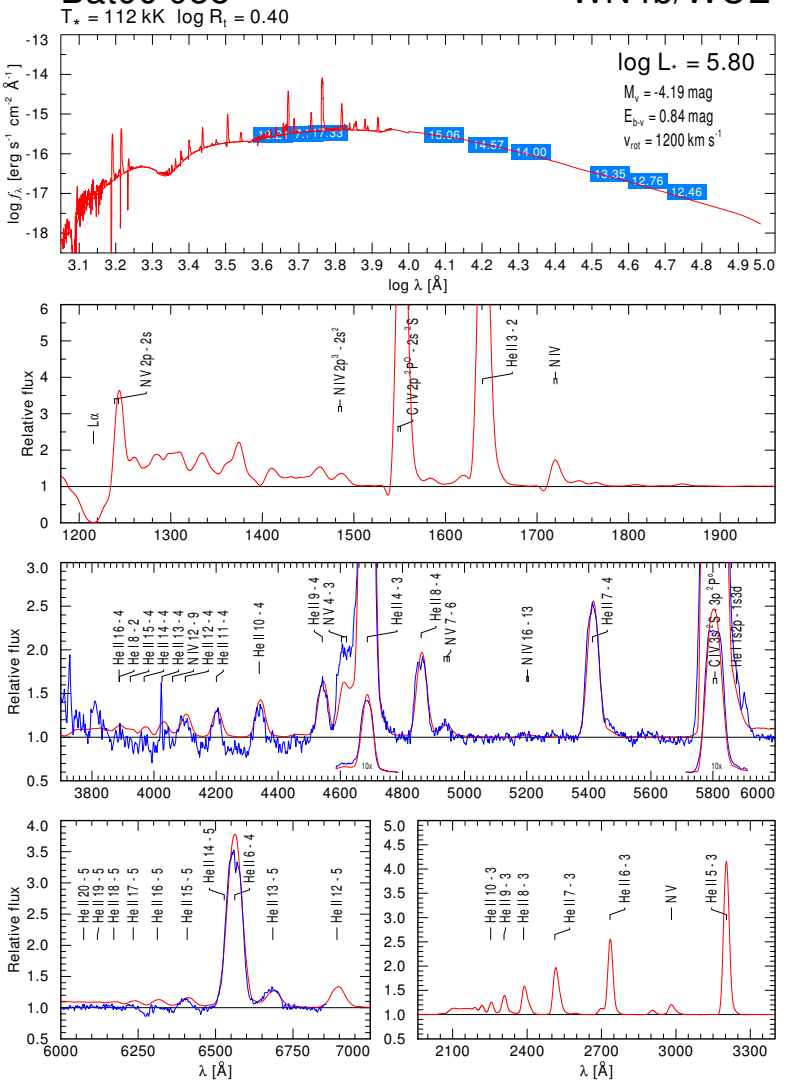
R. Hainich et al.: The Wolf-Rayet stars in the Large Magellanic Cloud

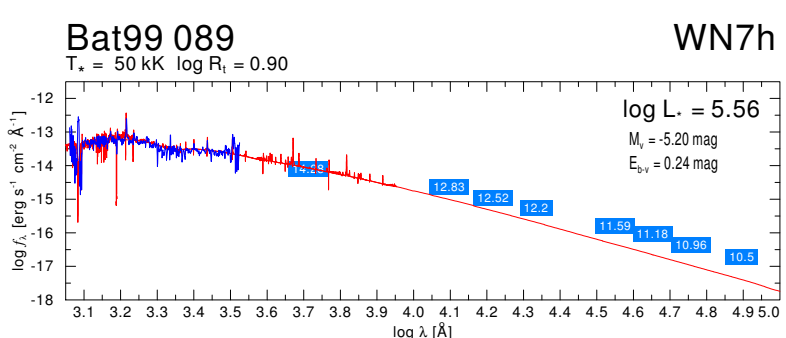

Bat99 091

WN6(h)
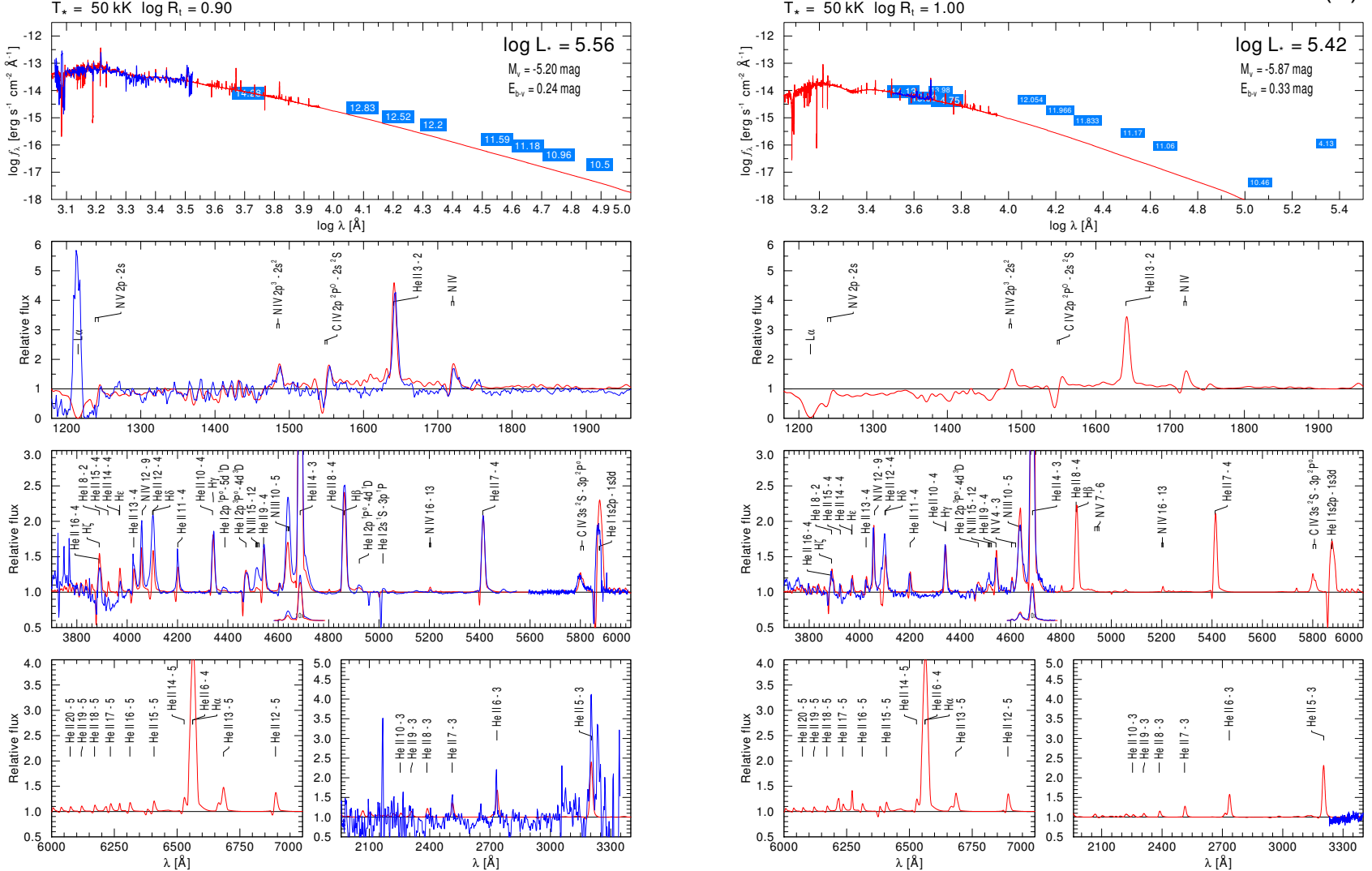

Fig. C.35. Spectral fit for BAT99 089 and BAT99 091.
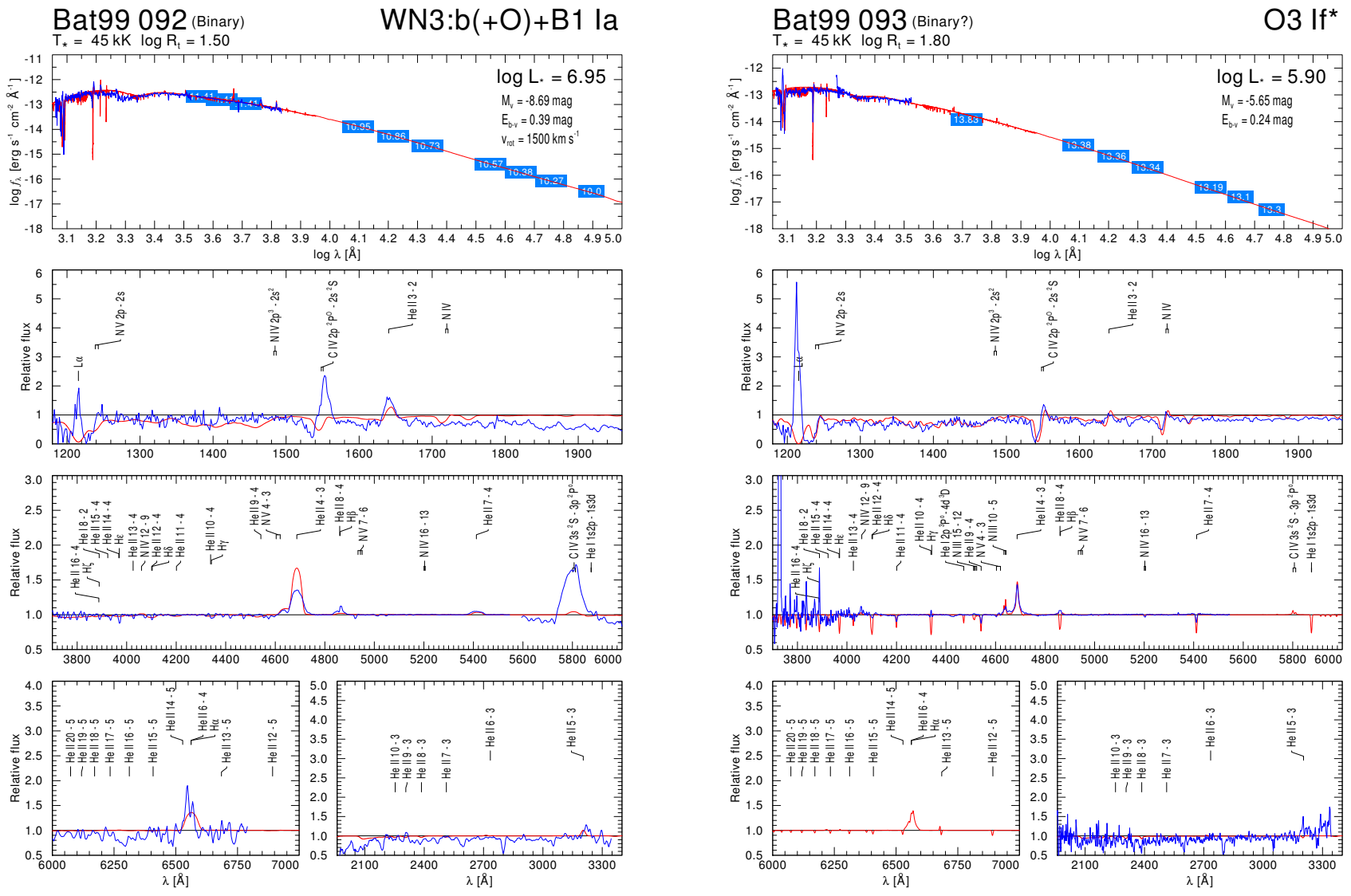

Fig. C.36. Spectral fit for BAT99 092 and BAT99 093. 
Bat99 094
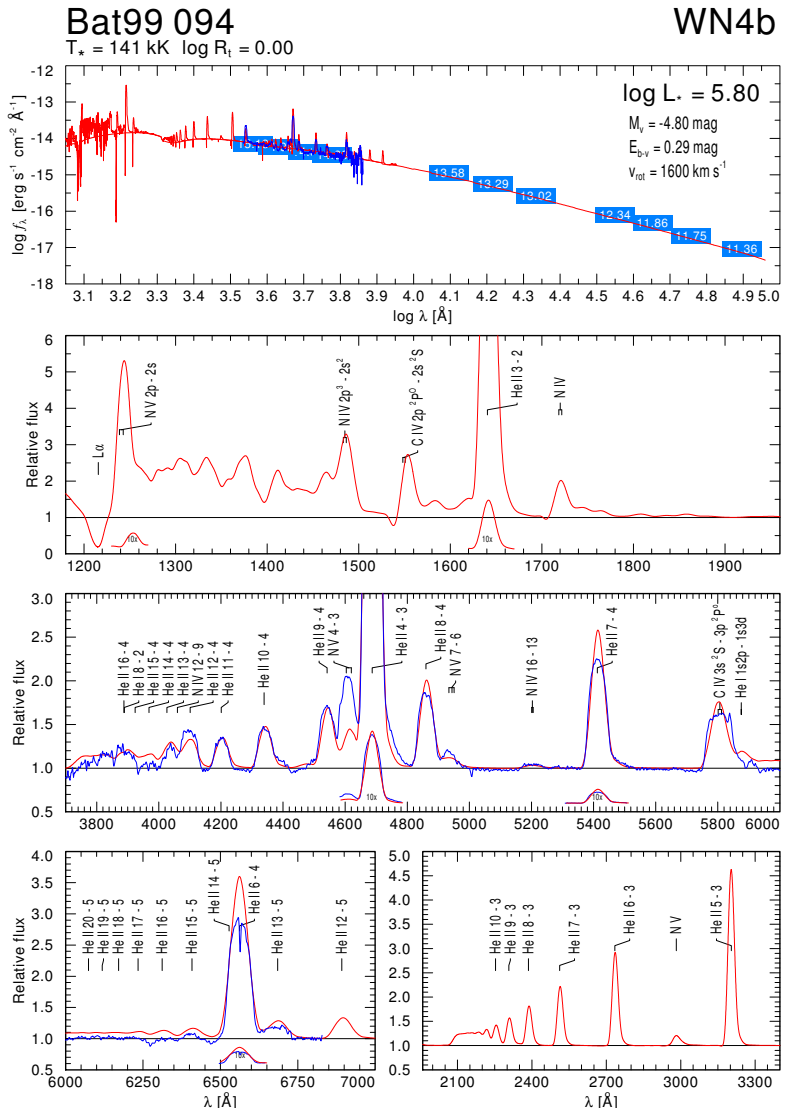

Fig. C.37. Spectral fit for BAT99 094 and BAT99 095.
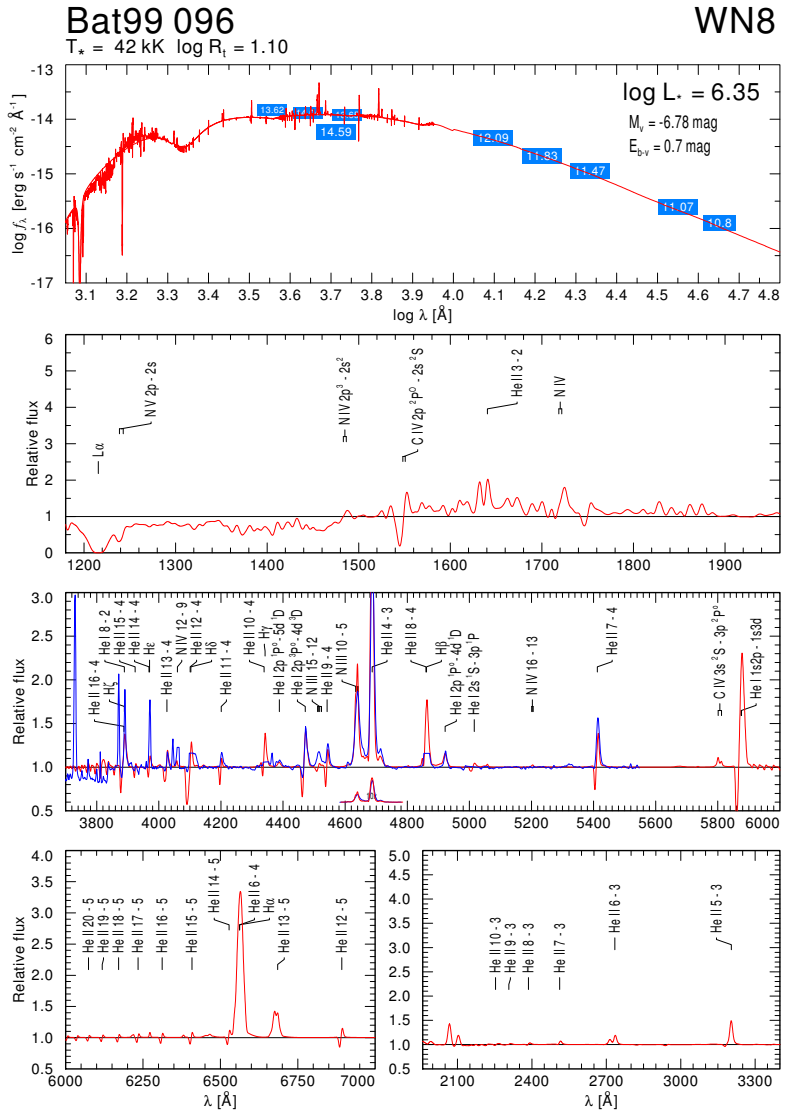

Fig. C.38. Spectral fit for BAT99 096 and BAT99 097.
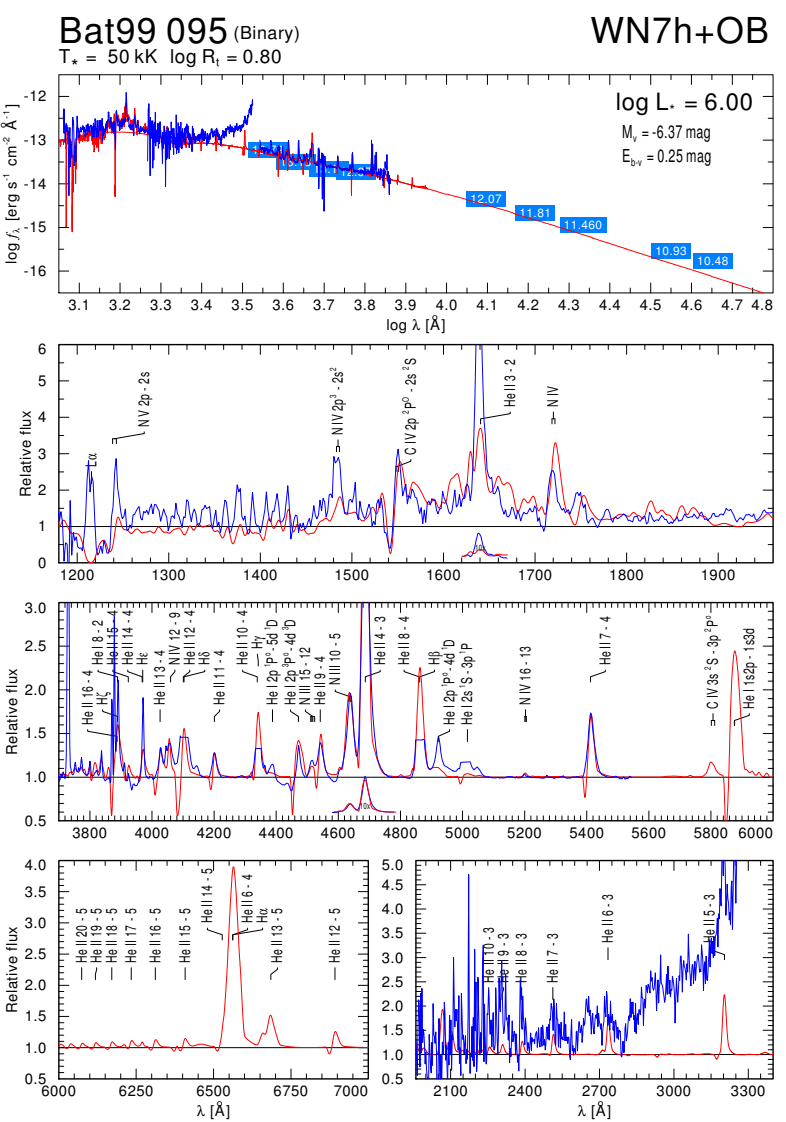

Bat99 097

O3.5 If*/WN7
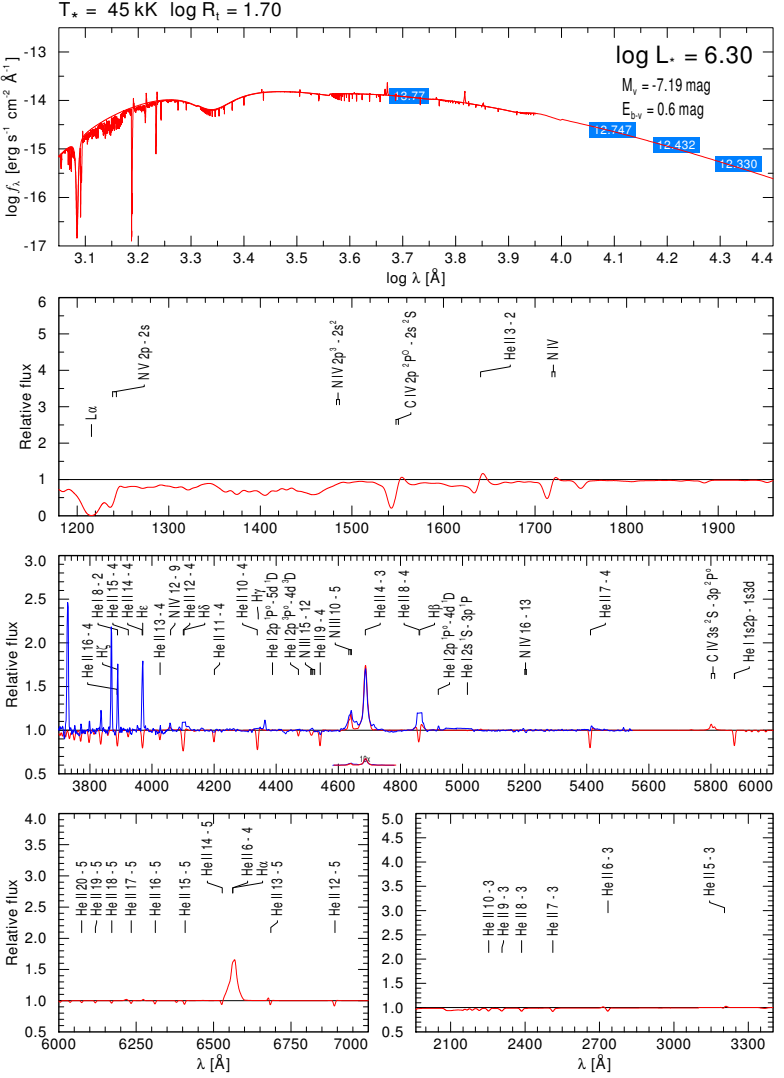
R. Hainich et al.: The Wolf-Rayet stars in the Large Magellanic Cloud

Bat99 098

WN6
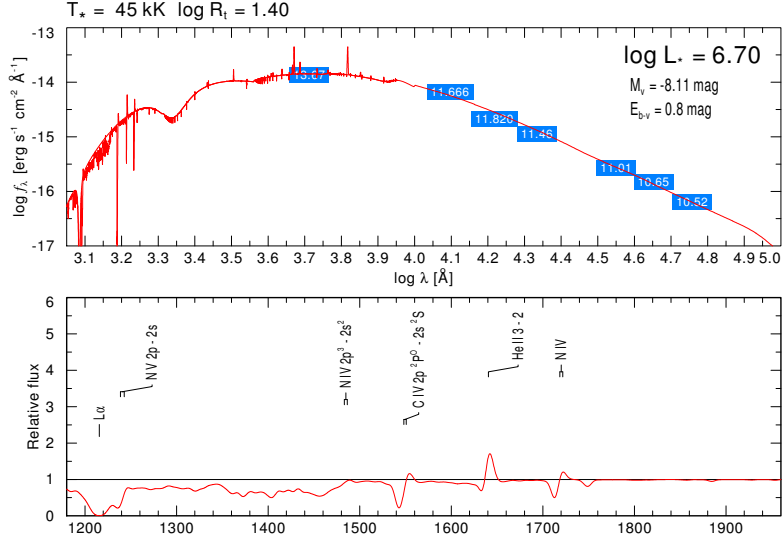

$\begin{array}{llll}3.0 & \\ 2.5 & \end{array}$

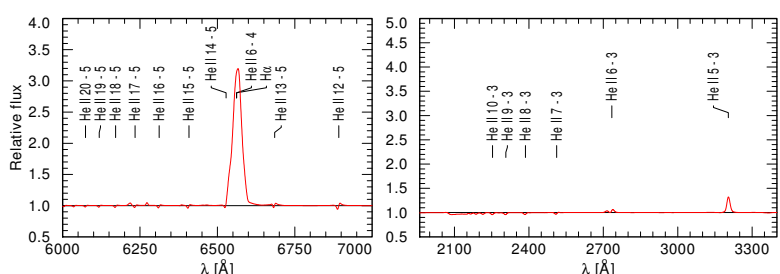

Fig. C.39. Spectral fit for BAT99 098 and BAT99 099.
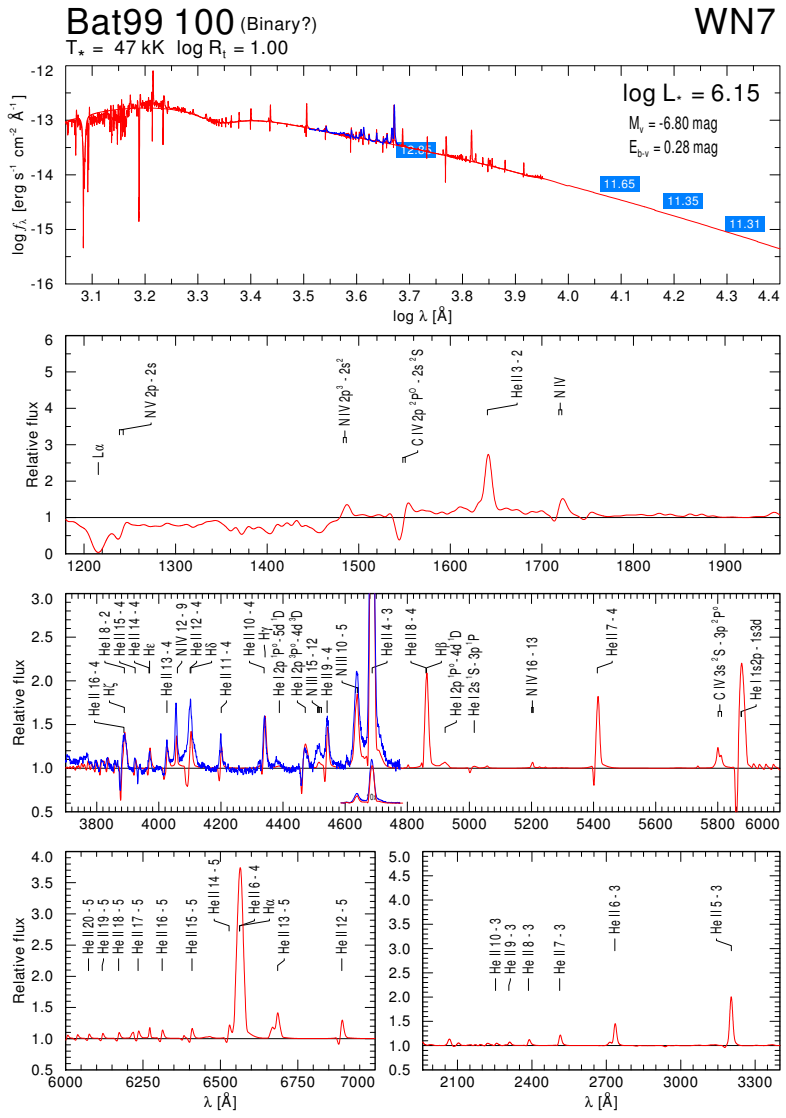

Fig. C.40. Spectral fit for BAT99 100 and BAT99 102.
Bat99 099 (Binary)
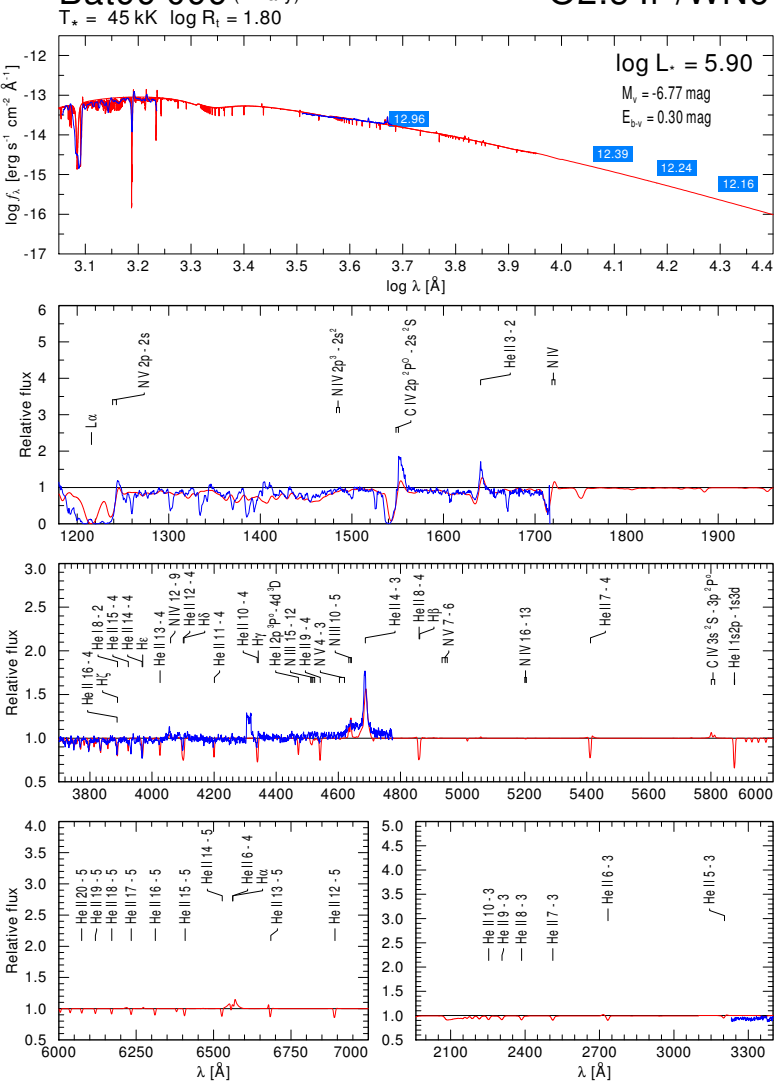


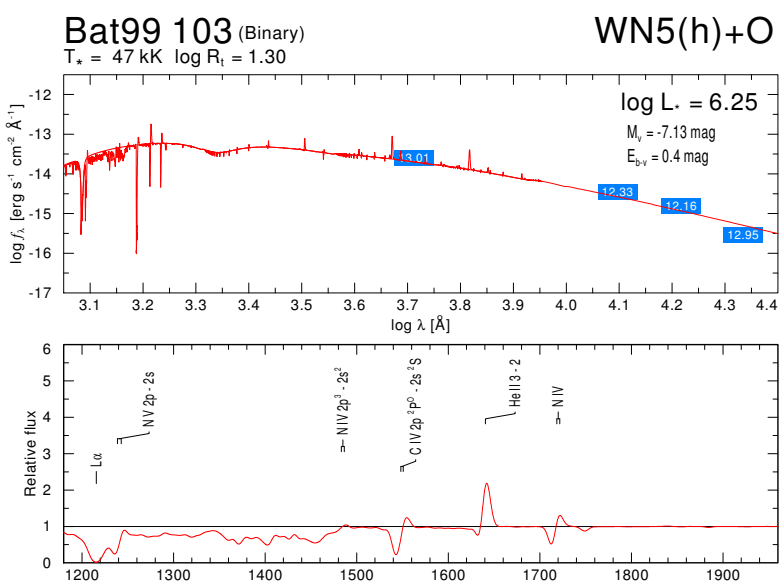

Bat99 104

O2 If*/WN5
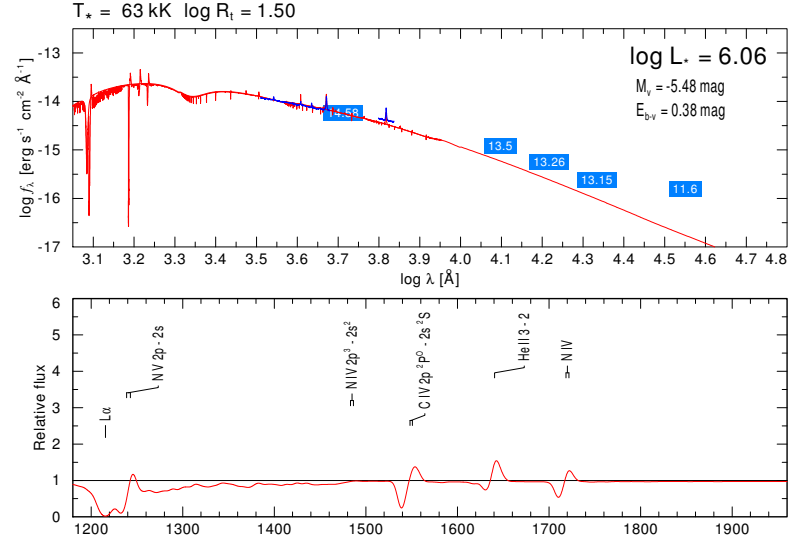

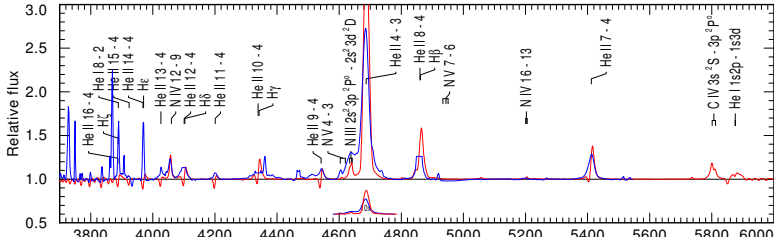

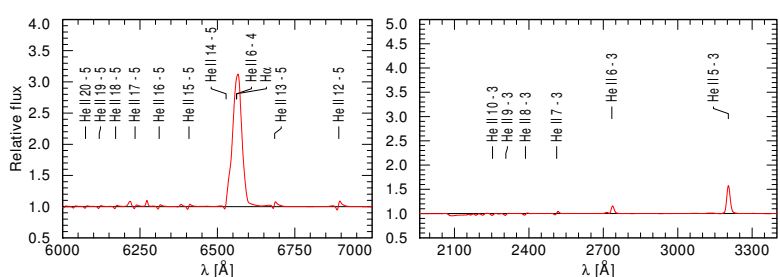

Fig. C.41. Spectral fit for BAT99 103 and BAT99 104.
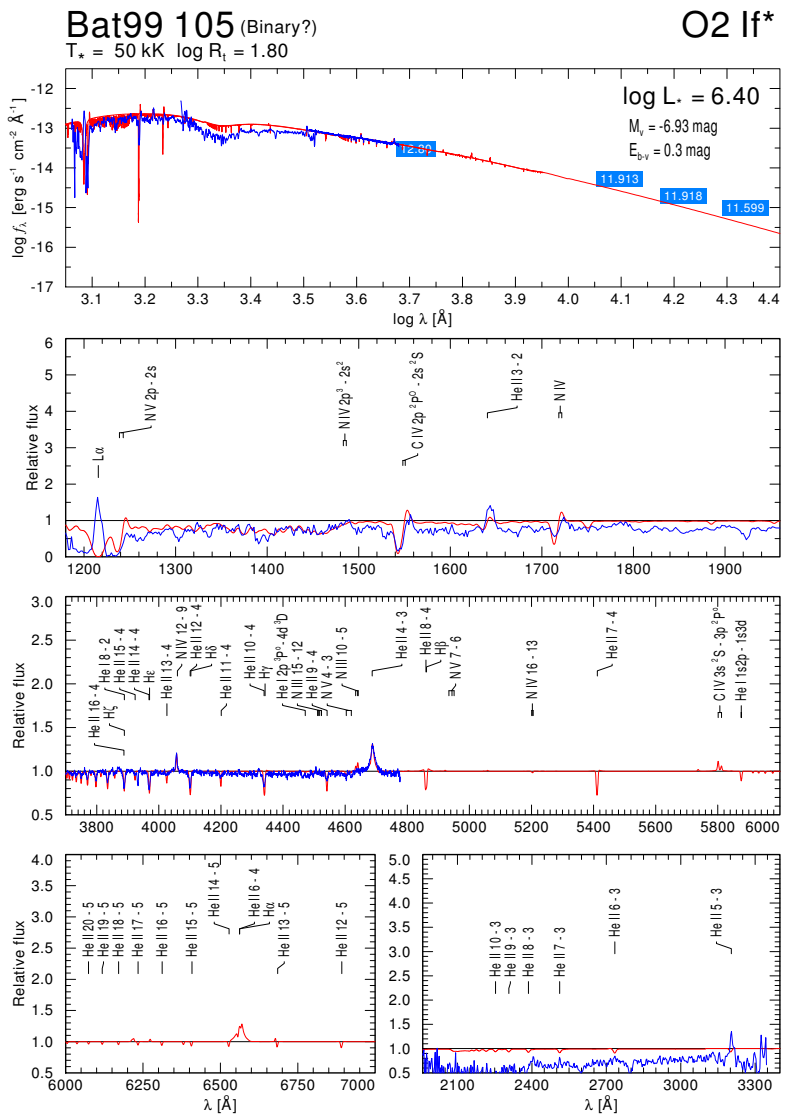

Fig. C.42. Spectral fit for BAT99 105 and BAT99 106.
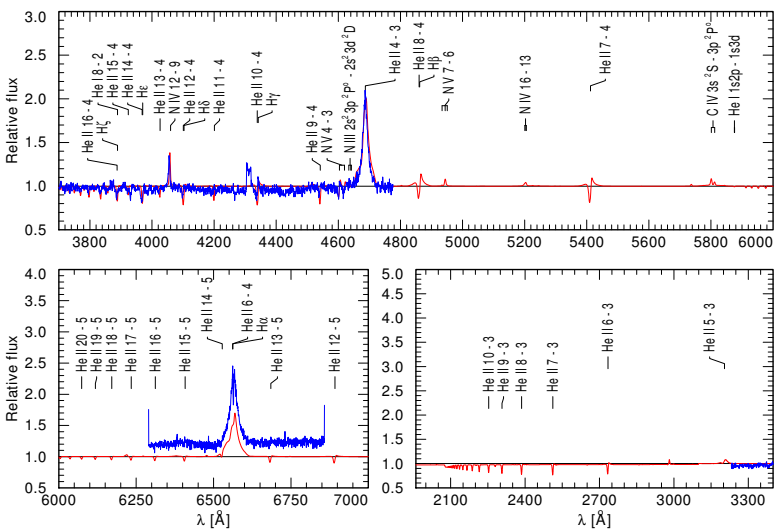

Bat99 106
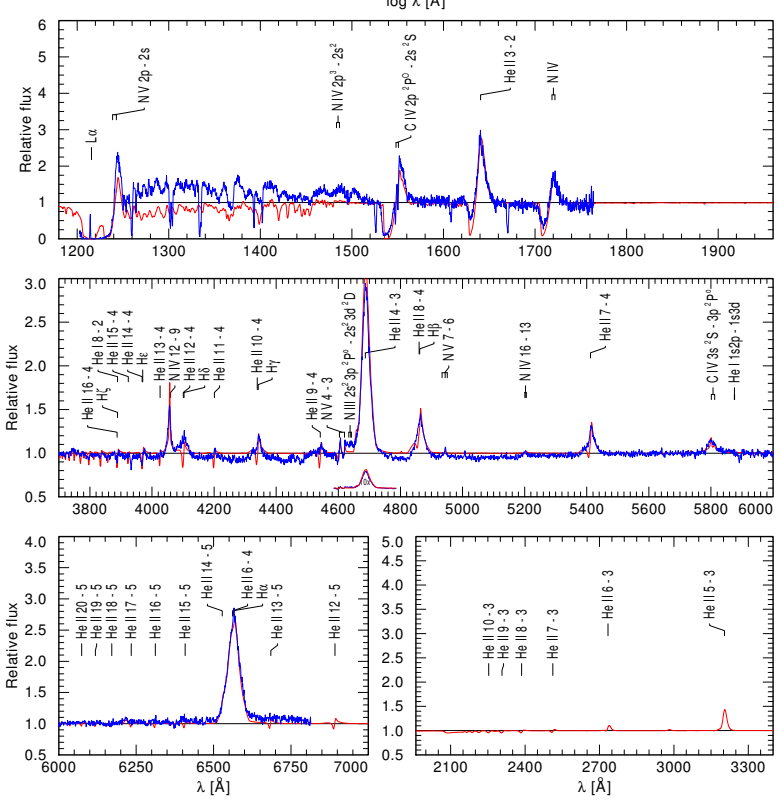

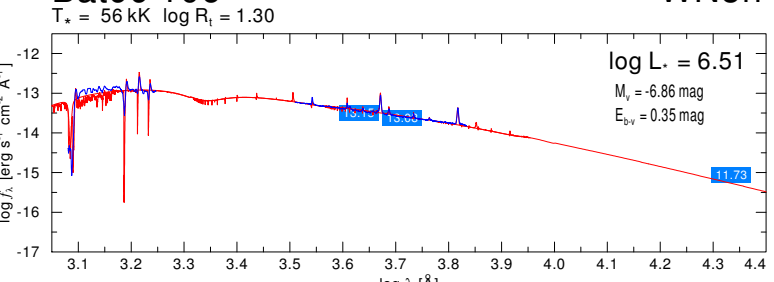


R. Hainich et al.: The Wolf-Rayet stars in the Large Magellanic Cloud

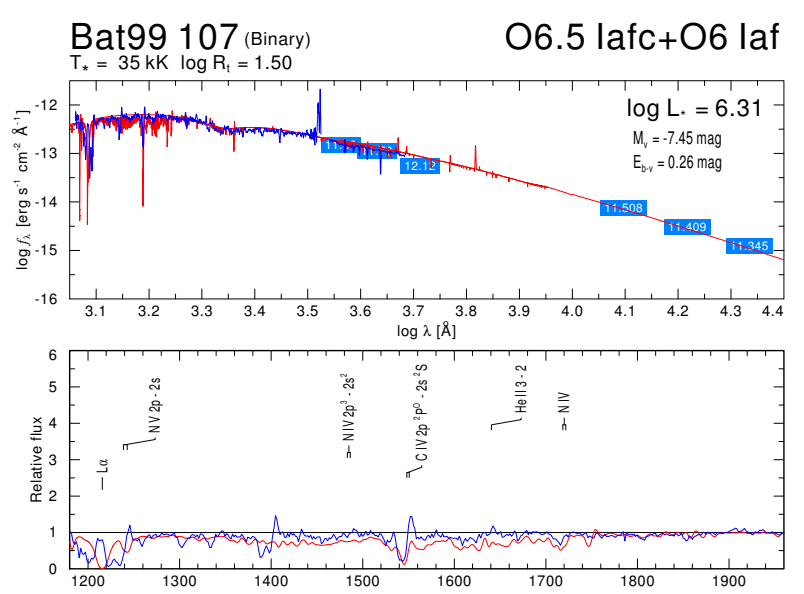

Bat99 108

WN5h

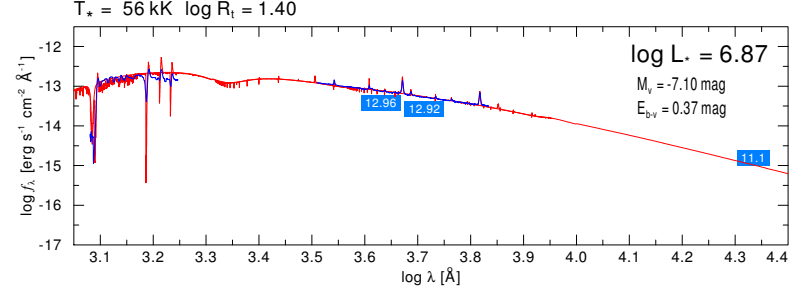

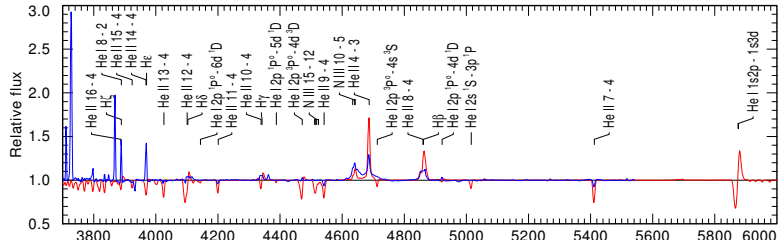
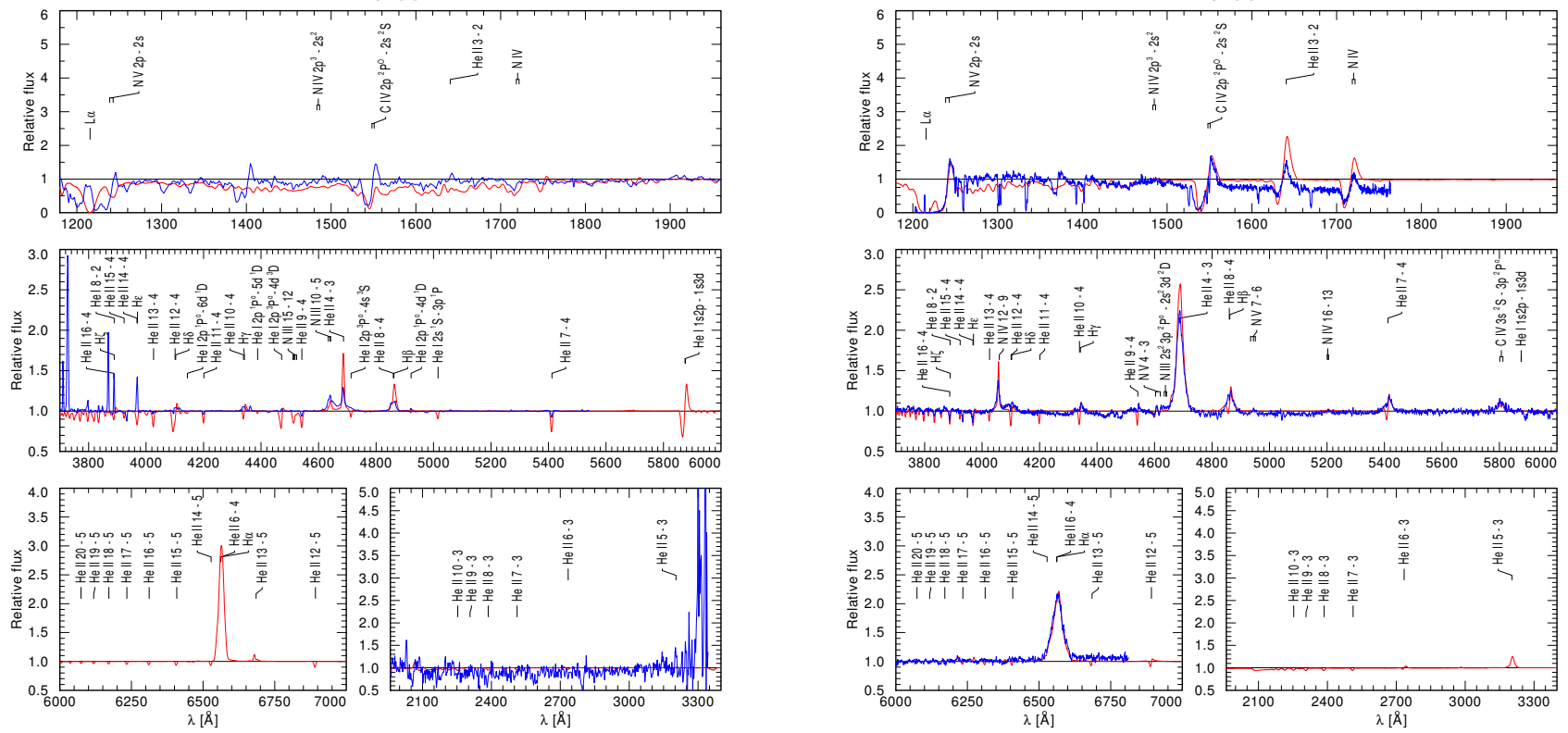

Fig. C.43. Spectral fit for BAT99 107 and BAT99 108.
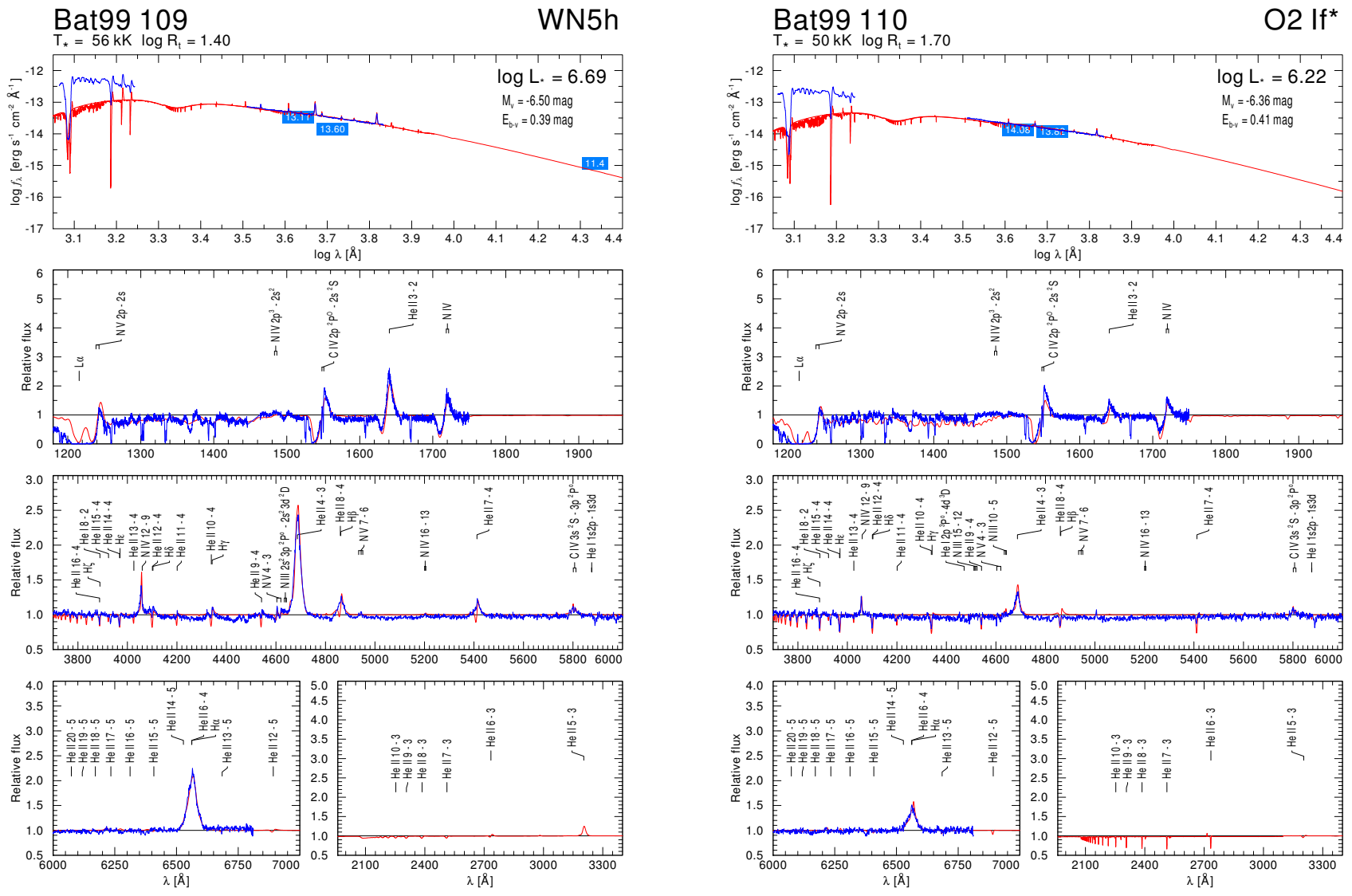

Fig. C.44. Spectral fit for BAT99 109 and BAT99 110. 
Bat99 111 (Binary?)
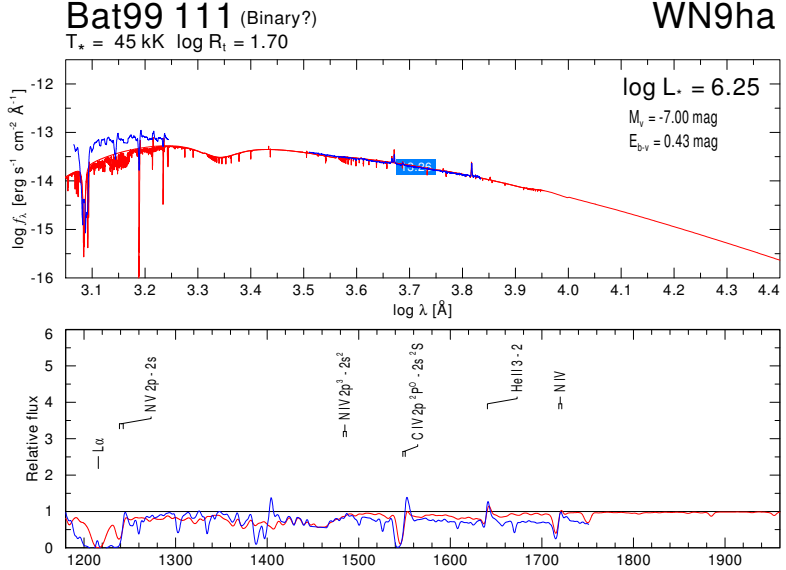

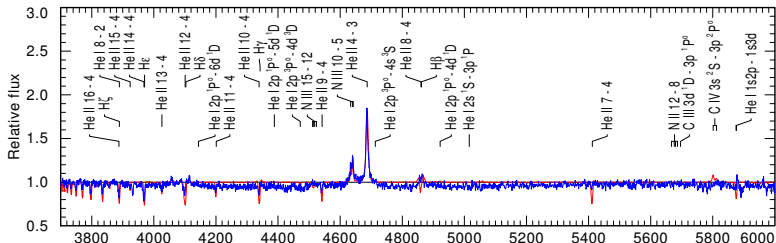

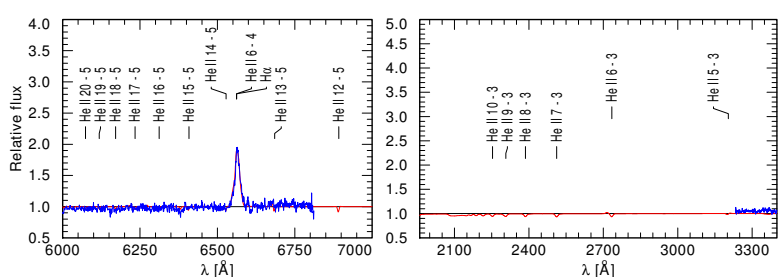

Fig. C.45. Spectral fit for BAT99 111 and BAT99 112.

Bat99 113 (Binary
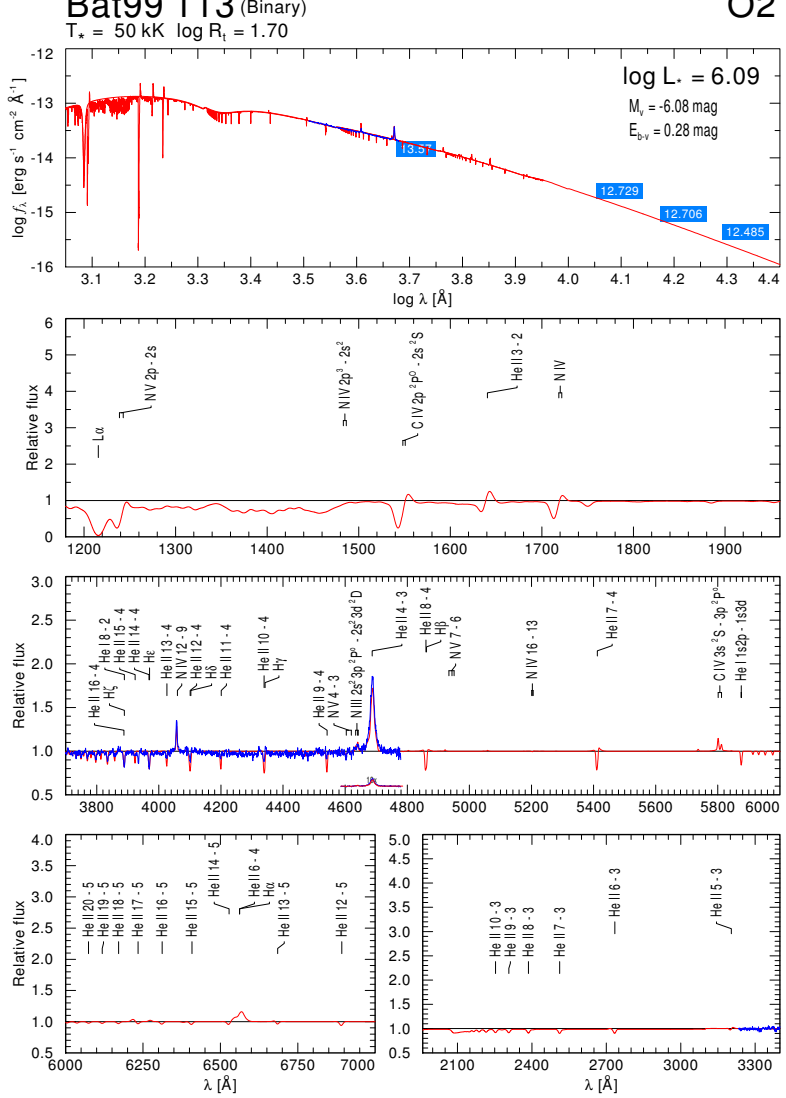

Fig. C.46. Spectral fit for BAT99 113 and BAT99 114 .
Bat99 112 (Binary?)

WN5h
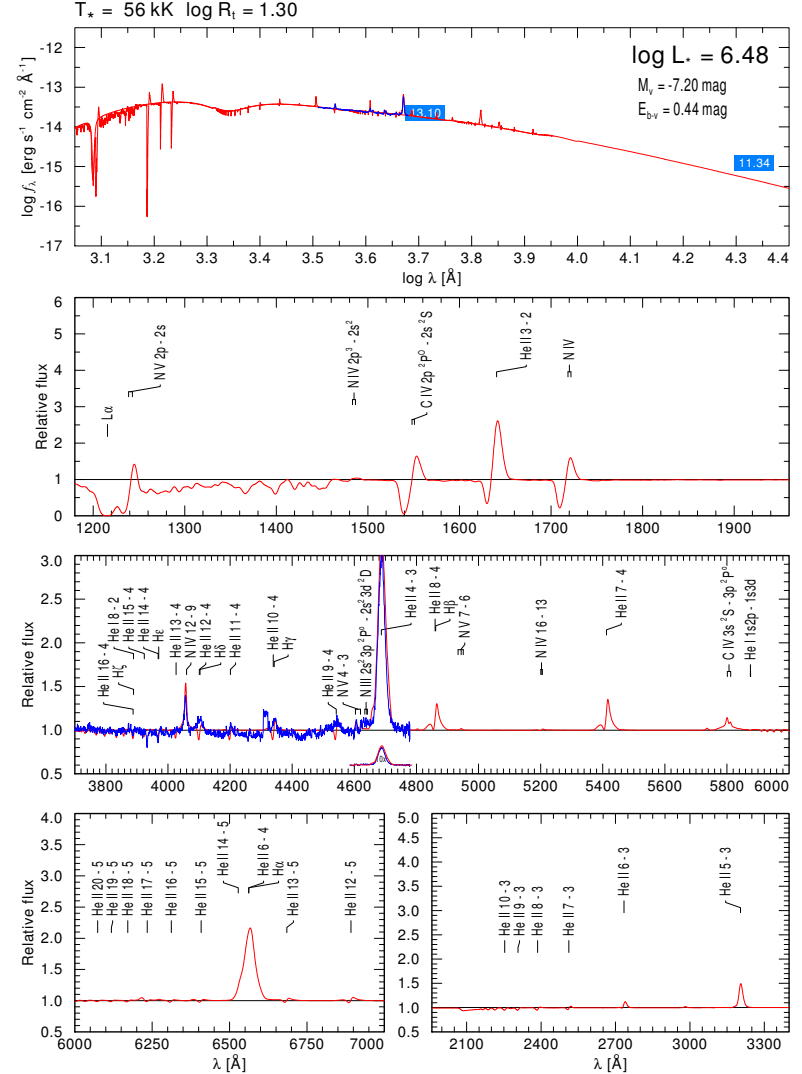

Bat99 114 (Binary?)
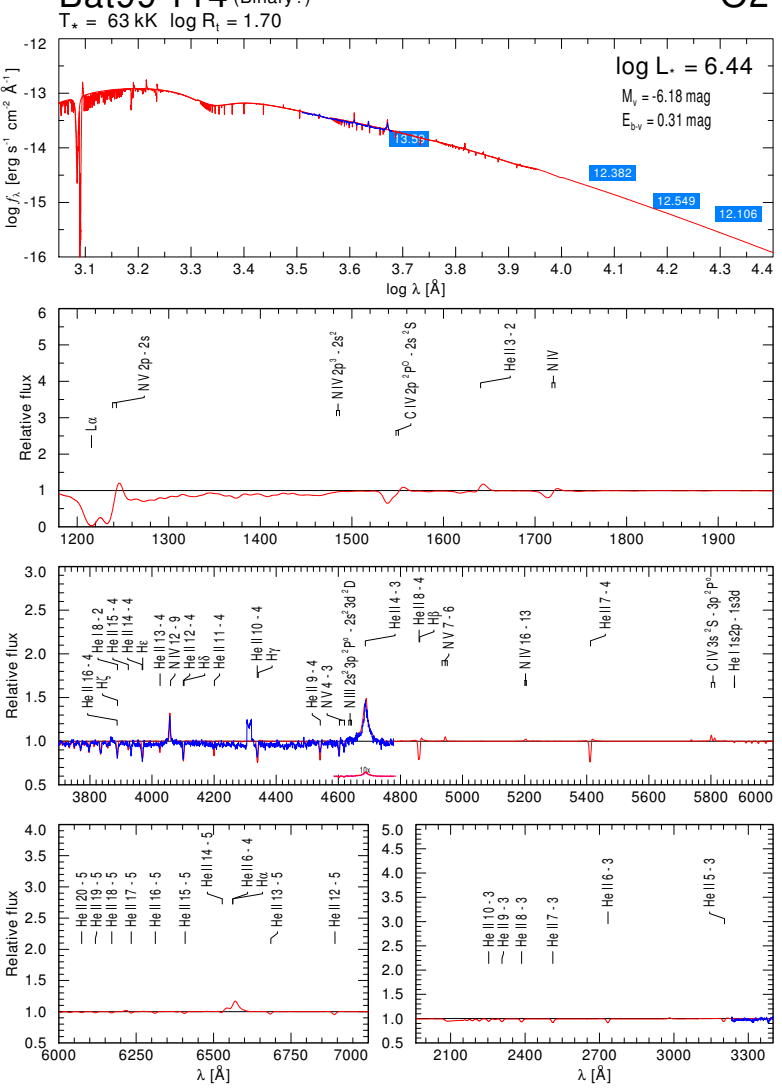
R. Hainich et al.: The Wolf-Rayet stars in the Large Magellanic Cloud

Bat99 116 (Binary?)
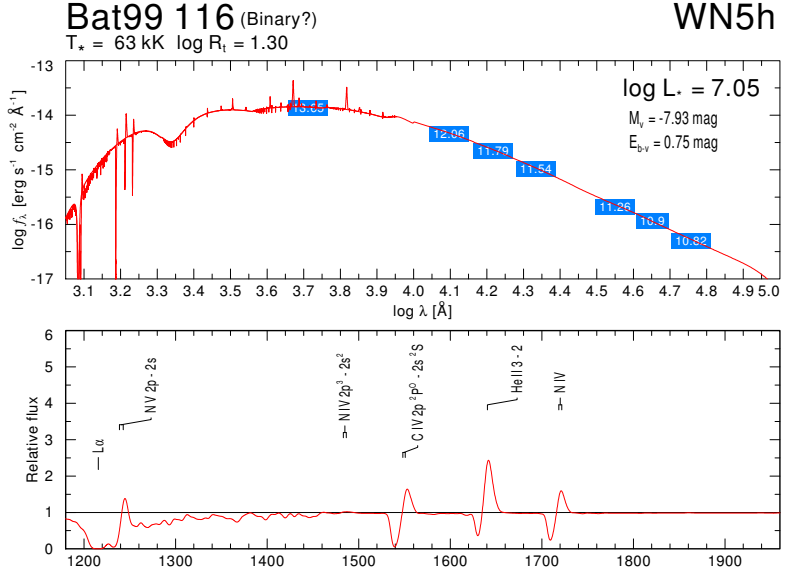

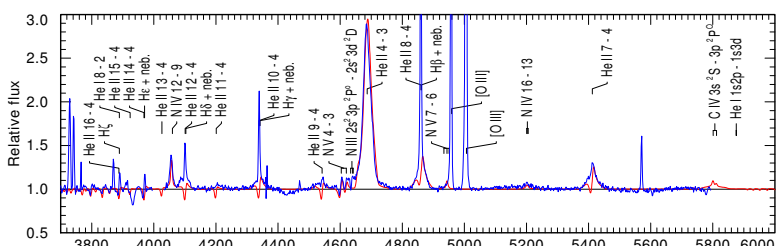

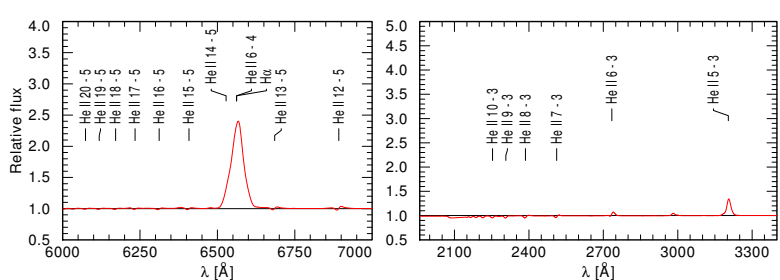

Fig. C.47. Spectral fit for BAT99 116 and BAT99 117.
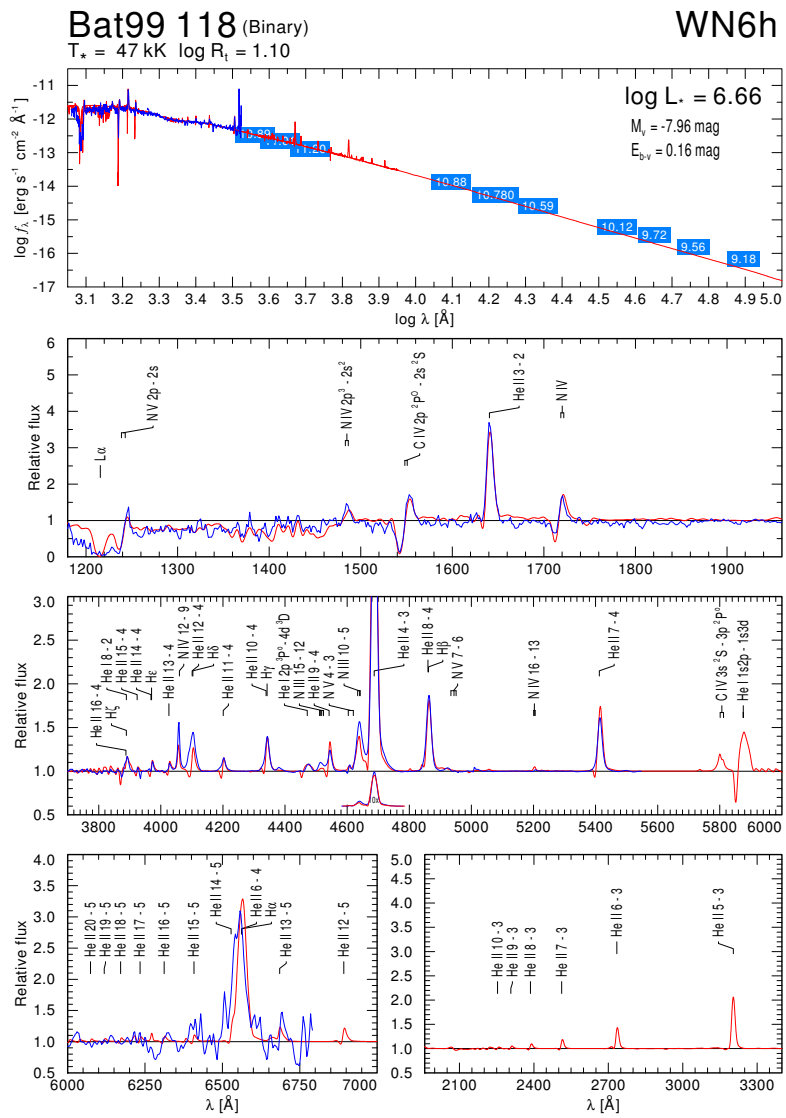

Fig. C.48. Spectral fit for BAT99 118 and BAT99 119.
Bat99 117

WN5ha
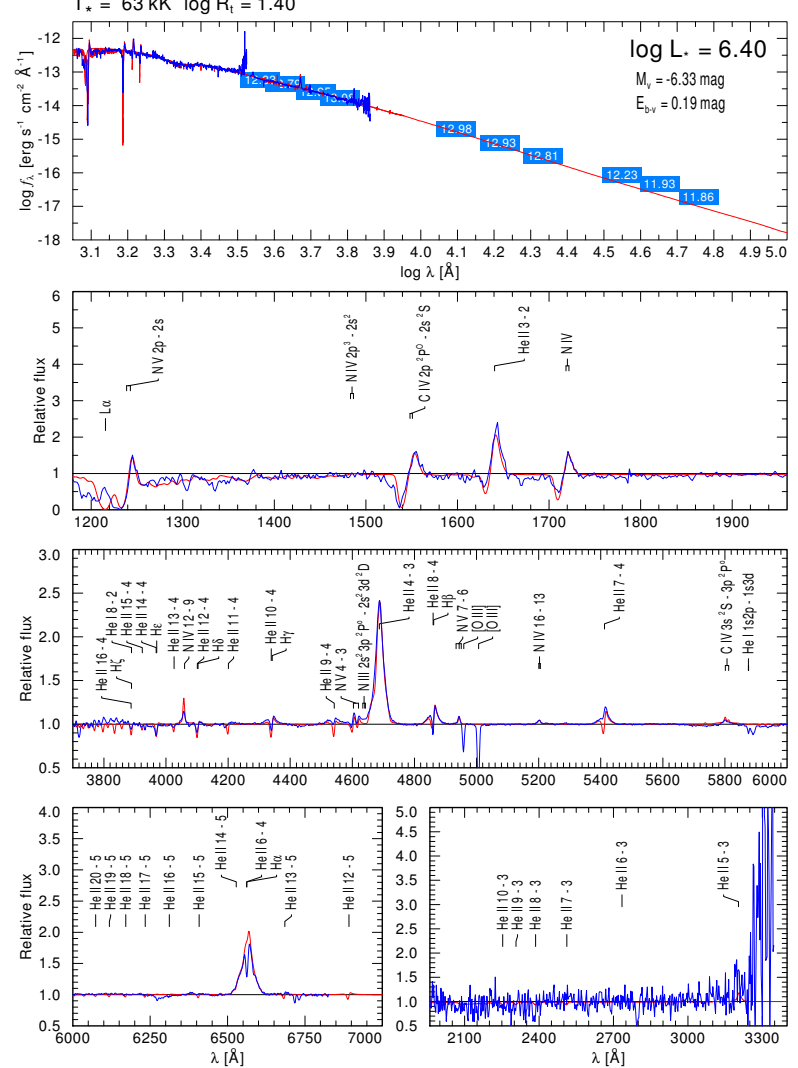
Bat99 120
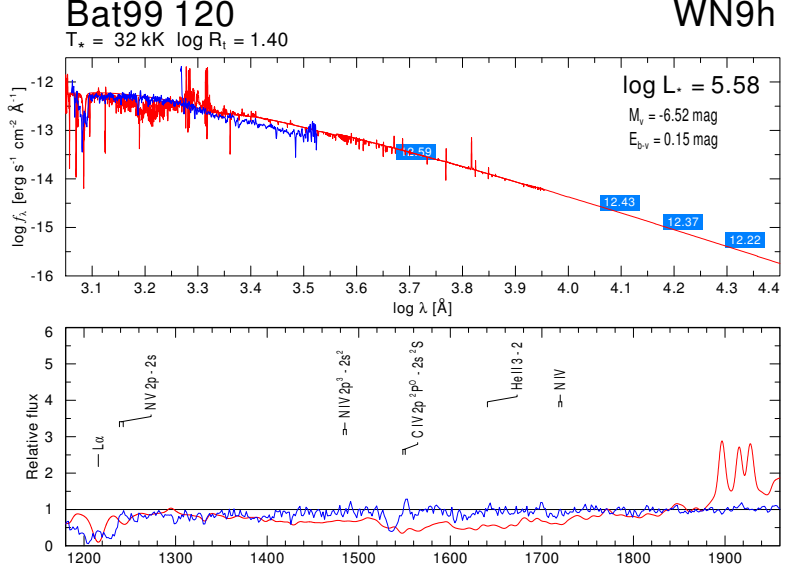

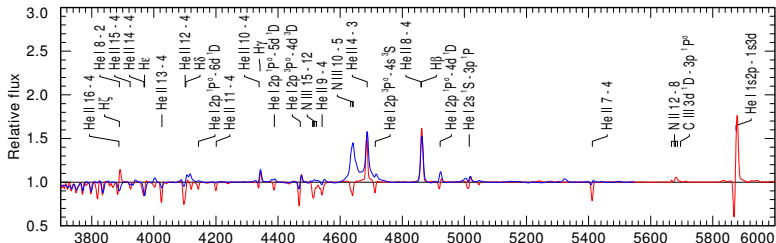

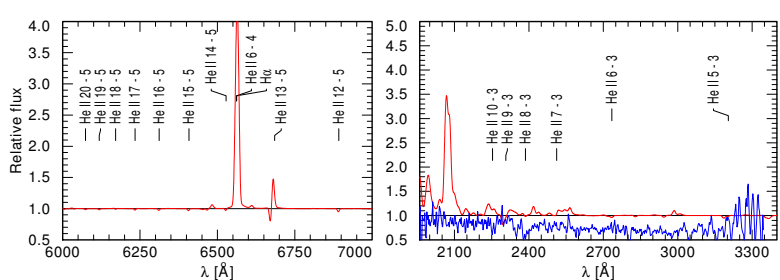

Fig. C.49. Spectral fit for BAT99 120 and BAT99 122.
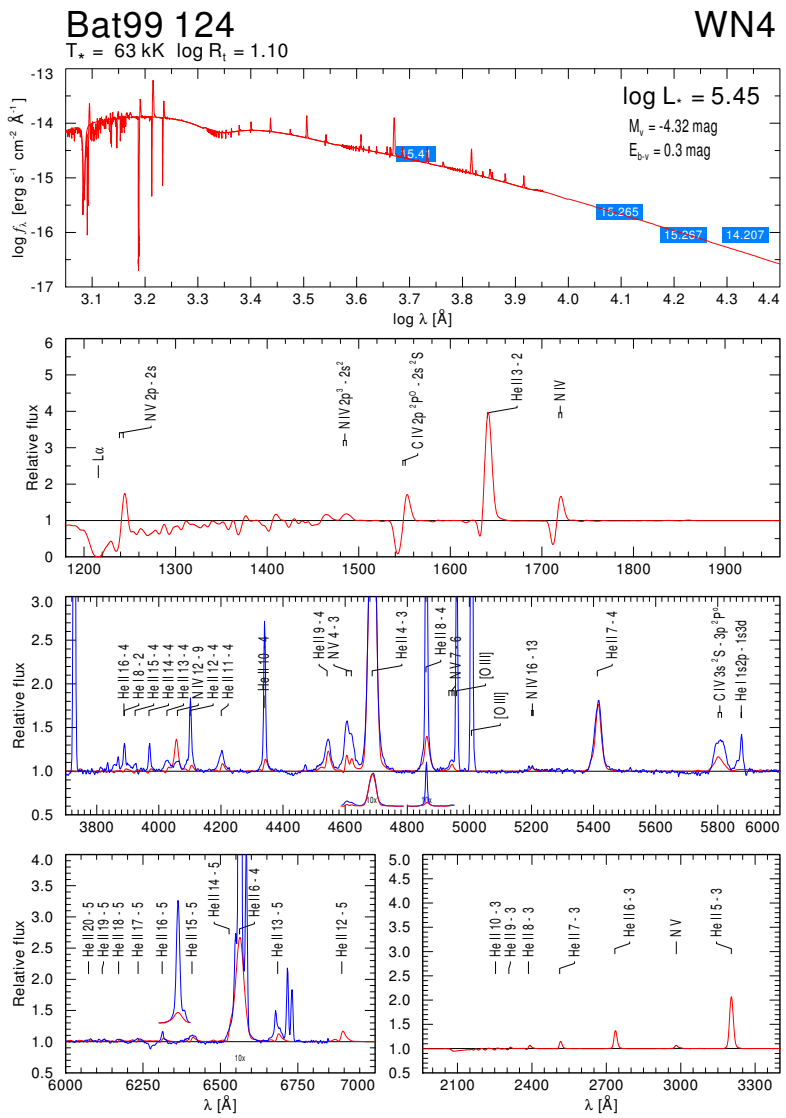

Fig. C.50. Spectral fit for BAT99 124 and BAT99 126.
Bat99 122

WN5h
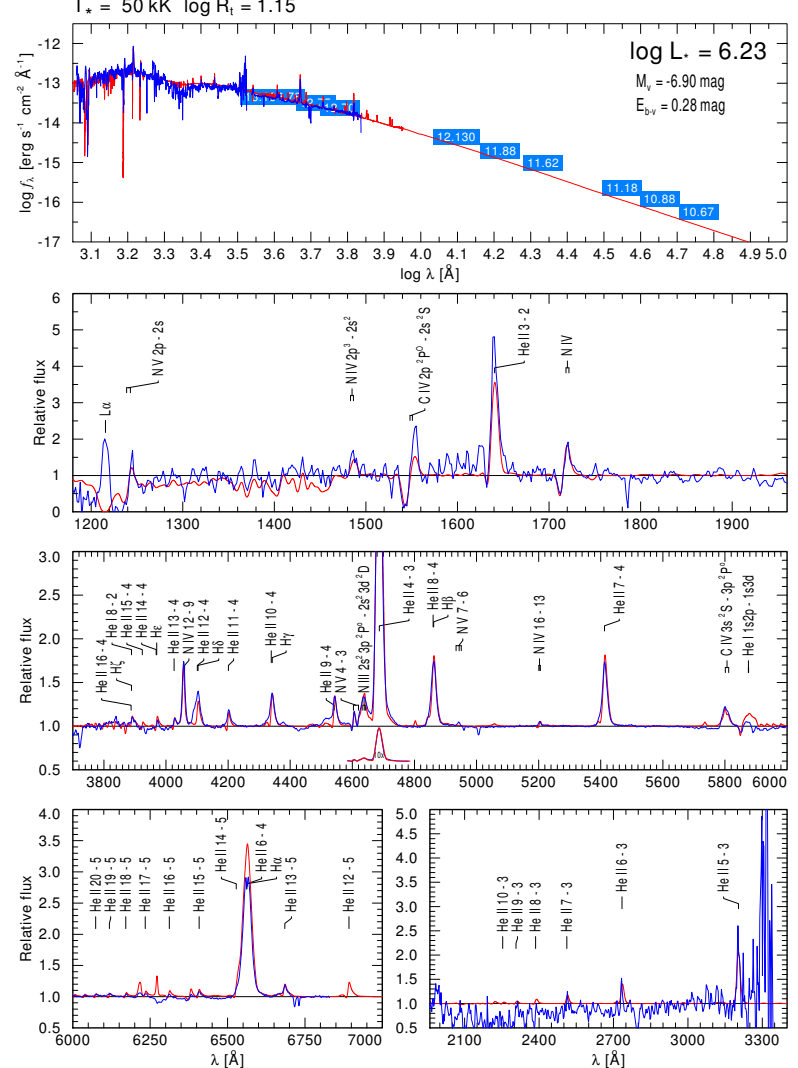
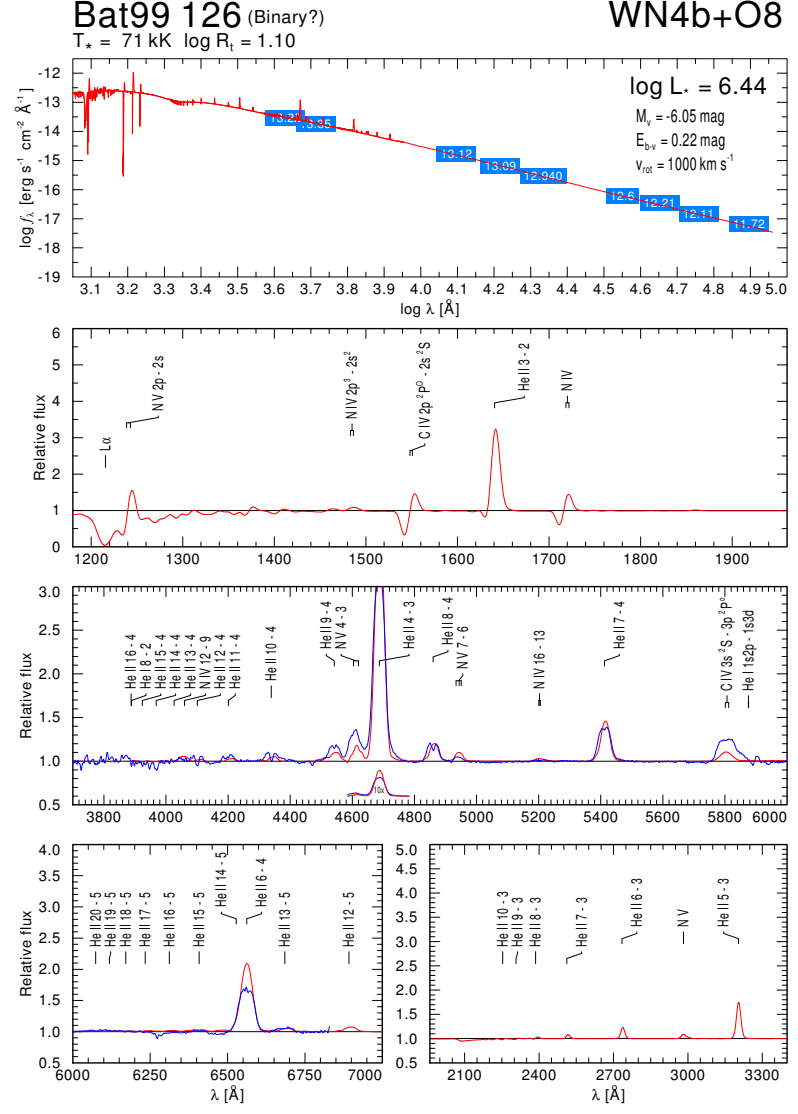

A27, page 60 of 62 
R. Hainich et al.: The Wolf-Rayet stars in the Large Magellanic Cloud

Bat99 128
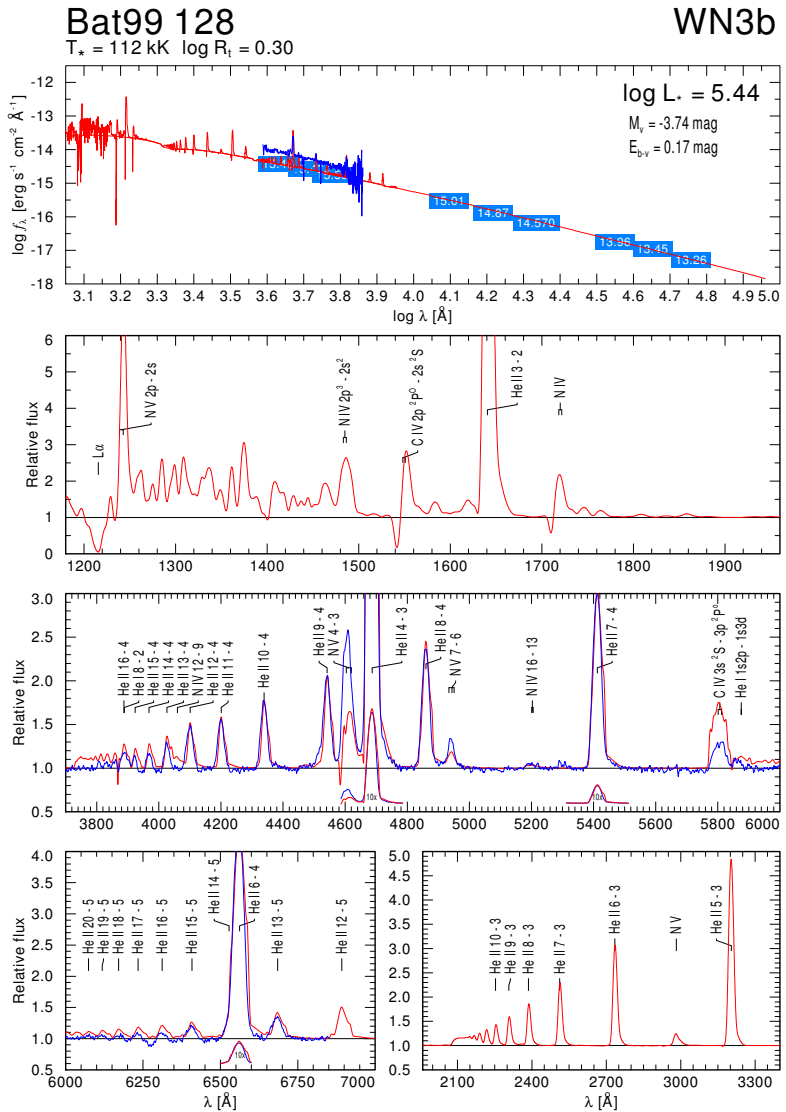

Fig. C.51. Spectral fit for BAT99 128 and BAT99 129.
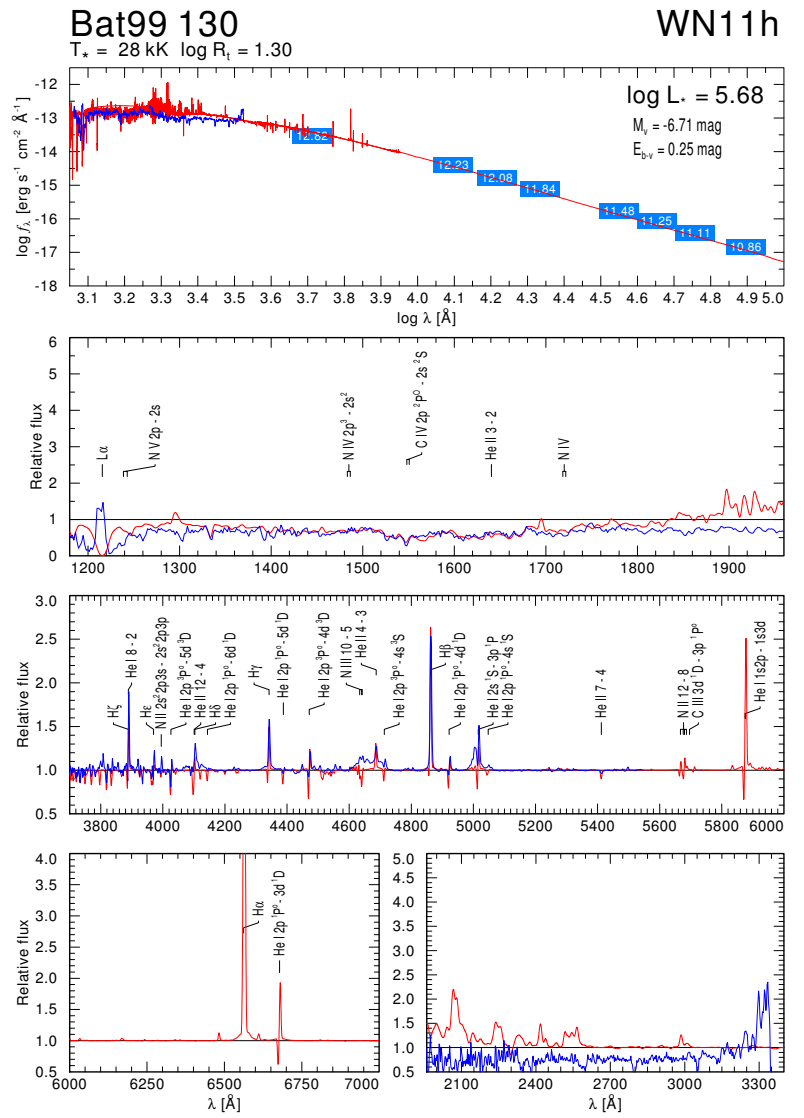

Bat99 129 (Binary)
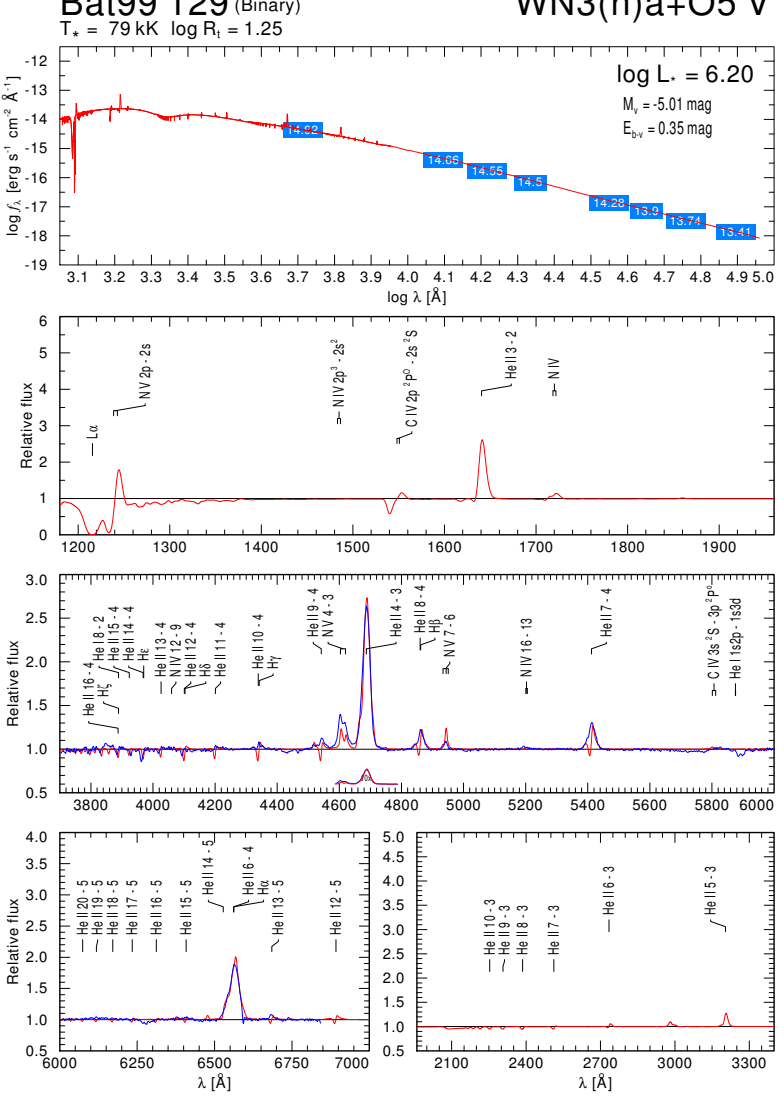

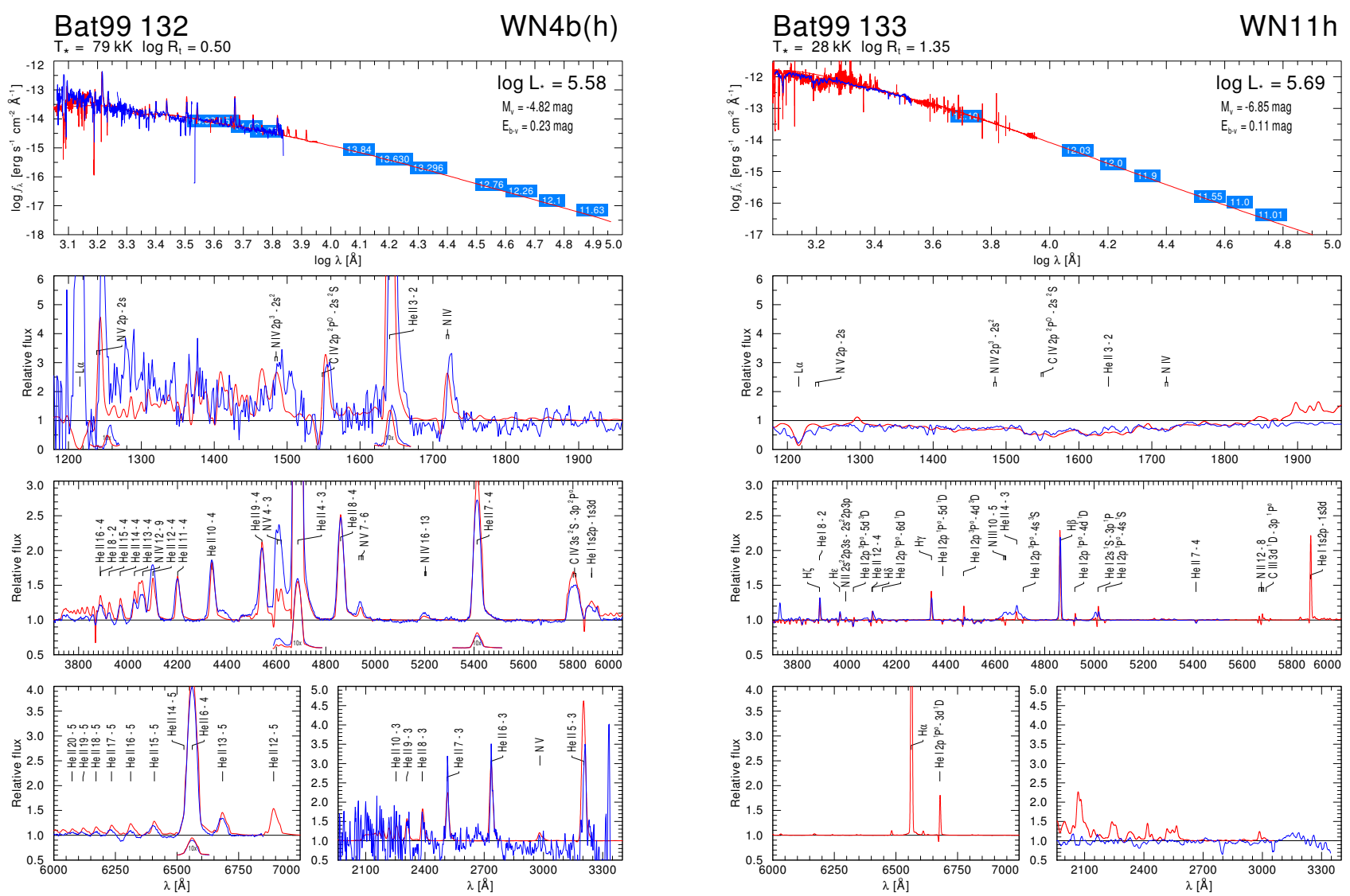

Fig. C.53. Spectral fit for BAT99 132 and BAT99 133.

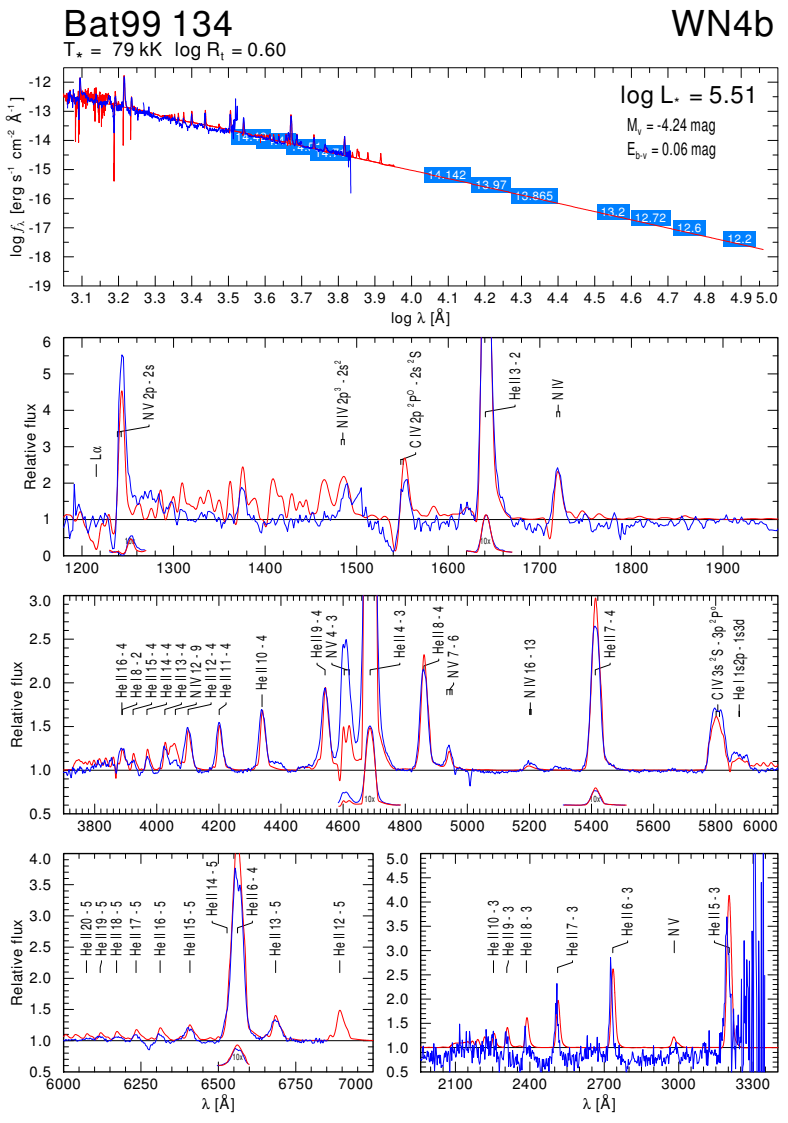

Fig. C.54. Spectral fit for BAT99 134. 